\title{
Semi-parametric Generalized Log-Gamma Regression Models
}

\author{
Carlos Alberto Cardozo Delgado
}

THESES PRESENTED TO THE

INSTITUTE OF MATHEMATICS AND STATISTICS

OF THE

UNIVERSITY OF SÃO PAULO

TO

FULFILL THE REQUIREMENTS OF THE DEGREE

$\mathrm{OF}$

DOCTOR OF SCIENCE

Graduate Program: Statistics

Advisor: Ph.D. Gilberto Alvarenga Paula

During this project the author was supported by the agency

$\mathrm{CNPq}$ - Brazil

and Colciencias - Colombia.

São Paulo, March 2018 


\section{Dedication}

To my mom María Antonia for her unconditional love and wisedom. To share with me the passion about knowledge.

To my wife Sandra, a great support point over these years.

To my kids, Juan David and Ana María, my best examples of how to learn of the life and don't surrender. 


\section{Acknowledgements}

To me is obvious that this work is a crystallization of countless hints, moments and subtle ideas that many people share with me. So, I would like to thank all of you. A special mention to:

- Gilberto A. Paula for the opportunity to work under his guidance, his continuous challenges and his confidence.

- Viviana Giampaoli and Eliane Pinheiros because their observations in my qualify exam help me to improve this work.

- Luis H. Vanegas his illuminating conversations about classic statistics and semi-parametric models were essentials for this project.

- Liliana Blanco C. and Rodrigo de Castro K. for their excellence teaching, full of precision and clarity. These qualities shaped my mind.

- Yuri M. Suhov and Anatoli Iambartsev for let me know a little bit about information theory and quantum statistical mechanics.

- My fellows, Alejandro Roldán, Kishor K. Ramavarmaraja and Rogério de Assis Medeiros because always had time to discuss mathematical and cultural ideas.

- Finally, I would like to thank IME-USP, a great place to learn. 


\section{Abstract}

CARDOZO, C.A. Semi-parametric generalized log-gamma regression models. 2017. Theses (Ph.D.) - Institute of Mathematics and Statistics, University of Sao Paulo, SP, 2017.

The central objective of this work is to develop statistical tools for semiparametric regression models with generalized log-gamma errors under the presence of censored and uncensored observations. The estimates of the parameters are obtained through the multivariate version of Newton-Raphson algorithm and an adequate combination of Fisher Scoring and Backffitting algorithms. Through analytical tools and using simulations the properties of the penalized maximum likelihood estimators are studied. Some diagnostic techniques such as quantile and deviance-type residuals as well as local influence measures are derived. The methodologies are implemented in the statistical computational environment $\mathbf{R}$. The package sglg is developed. Finally, we give some applications of the models to real data.

Keywords: censored observations, generalized log-gamma distribution, maximum penalized likelihood estimation, natural cubic spline, P-splines, skewness, semi-parametric models. 


\section{Resumo}

CARDOZO, C.A. Modelos de regressão semiparamétricos com erros log-gamma generalizados. 2017.Tese (Doutorado) - Instituto de Matemática e Estatística, Universidade de São Paulo, São Paulo, 2017.

O objetivo central do trabalho é proporcionar ferramentas estatísticas para modelos de regressão semiparamétricos quando os erros seguem distribução loggamma generalizada na presença de observações censuradas ou não censuradas. A estimacão paramétrica e não paramétrica são realizadas através dos procedimentos Newton - Raphson, escore de Fisher e Backfitting (Gauss - Seidel). As propriedades assintóticas dos estimadores de máxima verossimilhança penalizada são estudadas em forma analítica, bem como através de simulações. Alguns procedimentos de diagnóstico são desenvolvidos, tais como resíduos tipo componente do desvio e resíduo quantílico, bem como medidas de influência local sob alguns esquemas usuais de perturbacão. Os principais procedimentos do presente trabalho são implementados no ambiente computacional $\mathbf{R}$, assim como algumas aplicações a dados reais são apresentadas.

Palavras-chave: distribuições assimétricas, distribuição log-gamma generalizada, estimação por máxima verossimilhança penalizada, modelos semiparamétricos, observacões censuradas, P-splines, spline cúbico natural. 


\section{Contents}

Dedication $\quad$ i

Acknowledgements

Abstract iii

Resumo iv

1 Introduction $\quad 1$

1.1 A brief review . . . . . . . . . . . . . . . . . 1

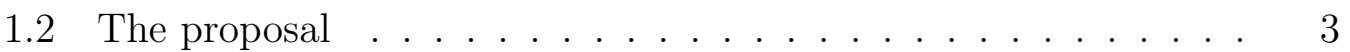

1.3 The structure ........................ 4

2 Generalized log-gamma family 5

2.1 Some precedents . . . . . . . . . . . . . . 5

2.2 The prototype density . . . . . . . . . . . . . 6

2.3 A tractable density . . . . . . . . . . . . . . 8

2.3.1 Special cases. . . . . . . . . . . . . . . . . . . . 9

2.4 Statistical properties . . . . . . . . . . . . . . . . . 10

2.4.1 A glimpse to the lifetime analysis . . . . . . . . . . . 19

2.4.2 Final remark ................ 21

3 Uncensored case $\quad 23$

3.1 Introduction . . . . . . . . . . . . . . . . . . . . 23

3.2 Partially linear generalized log-gamma regression models . . . . 24

3.2.1 The model . . . . . . . . . . . . . . . . . . 24

3.2.2 Approximating smooth functions . . . . . . . . . . . 24

3.2.3 Penalized log-likelihood function . . . . . . . . . . . 26

3.3 Parameter estimation . . . . . . . . . . . . . . . . . 27 
3.3.1 Penalized score function and penalized Fisher information matrix . . . . . . . . . . . . . . . 27

3.4 Effective degrees of freedom . . . . . . . . . . . . . . . 29

3.5 Smoothing parameter . . . . . . . . . . . . . 29

3.6 Model selection . . . . . . . . . . . . . . . . . . . 30

3.7 Extension for the additive case . . . . . . . . . . . . . . . 30

3.7.1 Approximating smooth functions . . . . . . . . . . 31

3.7.2 Penalized log-likelihood function . . . . . . . . . . . . 32

3.8 Parameter estimation . . . . . . . . . . . . . . . . . . 33

3.8.1 Penalized score function and penalized Fisher information matrix . . . . . . . . . . . . . . 33

3.8.2 Back-fitting algorithm . . . . . . . . . . . . 34

3.9 Effective degrees of freedom . . . . . . . . . . . . . . 36

3.10 Smoothing parameter . . . . . . . . . . . . . . . . . 37

3.11 Model selection . . . . . . . . . . . . . . . . . 37

3.12 Asymptotic statements . . . . . . . . . . . . . . . . 37

3.13 Confidence band for a non-parametric component . . . . . . . . 39

4 Censored case 40

4.1 Introduction . . . . . . . . . . . . . . . . . . 40

4.2 Censored partially linear generalized log-gamma regression models 41

4.2.1 The model . . . . . . . . . . . . . . . . . . 41

4.2.2 The likelihood . . . . . . . . . . . . . . . . 42

4.3 Penalized log-likelihood function . . . . . . . . . . . . . . 43

4.3.1 Parameter estimation . . . . . . . . . . . . . . 43

4.3.2 Penalized score function . . . . . . . . . . . . 44

4.4 Smoothing parameter . . . . . . . . . . . . . . . . . 45

4.5 Model selection . . . . . . . . . . . . . . . . . . 45

4.6 Extension for the additive case . . . . . . . . . . . . . . . . 46

4.7 The likelihood . . . . . . . . . . . . . . . . . 46

4.7.1 Penalized log-likelihood function . . . . . . . . . . . . 47

4.7.2 Parameter estimation . . . . . . . . . . . . . . . . 47

4.7.3 Penalized score function . . . . . . . . . . . . . . . 48

4.7.4 Penalized observed information matrix . . . . . . . . . . 49

4.8 Effective degrees of freedom . . . . . . . . . . . . . . . 53

4.9 Smoothing parameter . . . . . . . . . . . . . . 53

4.10 Model selection . . . . . . . . . . . . . . . . 54

4.11 Asymptotic statements . . . . . . . . . . . . . 54 
5 Diagnostic analysis $\quad 56$

5.1 Residual analysis . . . . . . . . . . . . 56

5.1 .1 Deviance residual . . . . . . . . . . . . . . . . 56

5.1 .2 Uncensored case . . . . . . . . . . . . . . . . . . . . . . 57

5.1 .3 Censored case . . . . . . . . . . . . . . . . . . 57

5.1 .4 Overall goodness-of-fit . . . . . . . . . . . . . . 57

5.2 Local influence . . . . . . . . . . . . . . . . . . . 58

5.3 Perturbation schemes . . . . . . . . . . . . . . . . . . 59

5.3.1 Case-weight perturbation: uncensored case . . . . . . . 60

5.3.2 Case-weight perturbation: censored case . . . . . . . . 60

5.3.3 Response perturbation: uncensored case . . . . . . . . . 61

5.3.4 Response perturbation: censored case . . . . . . . . . . . 62

6 The sglg package $\quad 64$

6.1 Introduction . . . . . . . . . . . . . . . . . . 64

6.1 .1 Function glg . . . . . . . . . . . . . . . . . . . . . 64

$6.1 .2 \quad$ Function $\operatorname{sglg} \ldots \ldots \ldots$. . . . . . . . . . . . . 6 66

6.1 .3 Function survglg . . . . . . . . . . . . . . . . . . . 70

6.1 .4 Function ssurvglg . . . . . . . . . . . . . . . . . . . 72

6.1 .5 Function shape . . . . . . . . . . . . . . . . 75

6.1 .6 Function AIC.sglg . . . . . . . . . . . . . . . 76

6.1 .7 Function SIC.sglg . . . . . . . . . . . . . . . . . 77

6.1 .8 Function envelope.sglg . . . . . . . . . . . . . . 77

6.1 .9 Function $\operatorname{rglg} \ldots \ldots \ldots \ldots$

6.1 .10 Some other functions . . . . . . . . . . . . . . 78

$\begin{array}{lll}7 & \text { Simulations and applications } & 80\end{array}$

7.1 Simulation studies . . . . . . . . . . . . . . . 80

7.1 .1 Case: $g l g \ldots \ldots$. . . . . . . . . . . . . . . . 80

7.1 .2 Case: $\operatorname{sglg} \ldots \ldots \ldots \ldots \ldots$

7.1 .3 Case: survglg . . . . . . . . . . . . . . . . . . . . . 83

7.1 .4 Case: ssurvglg . . . . . . . . . . . . . . . . . . . 85

7.1.5 How to select the starting values . . . . . . . . . . . 85

7.2 Diagnostic related grouping system, DRG . . . . . . . . 86

7.2.1 A preliminary analysis . . . . . . . . . . . . . 87

7.2 .2 The model . . . . . . . . . . . . . . . . . . . 88

7.3 Primary biliary cirrhosis . . . . . . . . . . . . . . . . . 92

7.3 .1 A preliminary analysis . . . . . . . . . . . 93 
7.3.2 The model . . . . . . . . . . . . . . . . . . 93

8 Conclusions $\quad 98$

8.1 Final considerations . . . . . . . . . . . . . . . . . . . . . 98

8.2 Future works . . . . . . . . . . . . . . . . . . . . . . . . 98

$\begin{array}{lr}\text { A Cubic splines and P-splines } & \mathbf{1 0 0}\end{array}$

A.1 Natural Cubic Splines . . . . . . . . . . . . . . . . . 100

A.2 Some properties . . . . . . . . . . . . . . . . . . 101

A.3 P-splines . . . . . . . . . . . . . . . . . . . . . . . 103

B Score function and Fisher information matrix: uncensored case106

B.1 Penalized score functions . . . . . . . . . . . . . . . . . 106

B.2 Penalized Hessian matrix . . . . . . . . . . . . . . . . . . . 108

B.3 Penalized Fisher Information . . . . . . . . . . . . . . . . 109

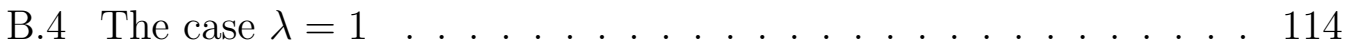

C Some useful derivations for the censored case $\mathbf{1 1 5}$

C.1 Penalized likelihood functions . . . . . . . . . . . . . . 115

C.2 Penalized score functions . . . . . . . . . . . . . . . . 116

C.3 Penalized observed information matrix . . . . . . . . . . 117

D Local influence analysis $\quad \mathbf{1 2 2}$

D.1 The perturbation schemes . . . . . . . . . . . . . . . . . 122

D.1.1 Case-weight perturbation: uncensored case . . . . . . . . 122

D.1.2 Case-weight perturbation: censored case . . . . . . . . . 123

D.1.3 Response perturbation: uncensored case . . . . . . . . . 124

D.1.4 Response perturbation: censored case . . . . . . . . . . 124

$\begin{array}{lr}\text { Bibliography } & 126\end{array}$ 


\section{List of Figures}

2.1 A diagrammatic view of the construction process of the $\operatorname{GLG}(\mu, \sigma, \lambda) . \quad 7$

2.2 Behavior of the p.d.f. of $\operatorname{GLG}(0,1, \lambda)$ for some $\lambda$ values. . . . . . 8

2.3 Behavior of the hazard function of $\operatorname{GLG}(0, \sigma, \lambda)$ for some values of $(\sigma, \lambda) \ldots \ldots \ldots \ldots 2 \ldots$

7.1 Diagram of possible selection of the starting values in the functions glg, sglg, survglg and ssurvglg. . . . . . . . . . . . . . . 86

7.2 Density of $\log ($ Cost) (left) and the dispersion graph between $\log ($ Cost $)$ and LOS for the patients in category 3 (right). . . . . 87

7.3 Overall goodness-of-fit statistic $\Upsilon$ (left), and the graph of $\hat{g}$ (LOS) as well as simultaneous $95 \%$ confidence intervals from the semiparametric generalized log-gamma model (7.1) fitted to the drg200 data (right). . . . . . . . . . . . . . . .

7.4 Dispersion graph between the deviance-type residual and the fitted value from the generalized log-gamma model fitted to the $\operatorname{drg} 2000$ data. . . . . . . . . . . . . . . . . . .

7.5 Normal probability plot of the deviance-type residual with a confidence band of $95 \%$ from the generalized log-gamma model fitted to the drg2000 data. . . . . . . . . . . . . . . . . .

7.6 Index plot based on the contribution of individual basic perturbation $E_{i}$ to the eigenvector $e_{\max }$ which gives the maximum conformal normal curvature (left) and of $B_{i}$ (right) under the caseweight perturbation scheme from the partially linear generalized log-gamma model fitted to the drg2000 data. . . . . . . . . . .

7.7 Behaviour of $\log ($ time $)$ and relationship with edema, stage and bili covariates. . . . . . . . . . . . . . . . 95

7.8 Estimated survival function (left) and the normal probability plot for the overall residual (right). . . . . . . . . . . . . . 96 
7.9 Index plot based on the contribution of individual basic perturbation $E_{i}$ to the eigenvector $e_{\max }$ which gives the maximum conformal normal curvature (left) and of $B_{i}$ (right) under the caseweight perturbation scheme from the partially linear generalized log-gamma model under the presence of censored observations adjusted to the PBC data. . . . . . . . . . . . . . . 97

A.1 An example of Gu's natural cubic spline base with knots in $0,0.1,0.2,0.3,0.4,0.5,0.6,0.7,0.8,0.9 \ldots \ldots \ldots \ldots 2$ 


\section{Chapter 1}

\section{Introduction}

We would like to begin with a brief state of the art of generalized gamma and log-gamma models, as well as some recent works inside the semi-parametric regression models.

\subsection{A brief review}

The study of the generalized gamma distribution began almost sixty years ago with a work by Stacy (1962). It was the first effort to treat in a unified way some of the most important distributions in reliability analysis, such as exponential, Weibull, gamma and log-normal distributions. Some properties of the generalized log-gamma distribution were studied in the sixties and seventies of the past century by Stacy and Mihram (1965); Hager and Bain (1970); Prentice (1974) and Lawless (1980). Some properties on the generalized log-gamma distribution are discussed in chapter 2.

Regression models with generalized log-gamma distributed errors have also been studied in the last decades. For example, the particular case of such models is the extreme value (type I) regression models, which were studied, for instance, by Lawless (1978) who studied confidence interval estimation in this kind of models and Paula and Rojas (1997) in the context of one-sided tests under uncensored observations. The extreme value type I distribution is also known as the distribution of the minimum, and is named in the gamlss $\mathrm{R}$ package Rigby and Stasinopoulos (2006) as reverse Gumbel distribution. In Ortega et al. (2003) was presented some estimation procedures and derived di- 
agnostic measures based on the local influence approach under the presence of censored observations, Cox et al. (2007) presented a taxonomy of the hazard function of generalized gamma distribution with application to study of survival after diagnostic of clinical AIDS during different phases of HIV therapy, whereas Zhang et al. (2008) assumed generalized log-gamma distributions for the random effect in linear mixed models. More recently, Ortega et al. (2008) compared three types of residuals in generalized log-gamma regression models under the presence of censored observations. In Fabio et al. (2012) is assumed a generalized log-gamma distribution for the random effect in random intercept Poisson models, whereas Ortega et al. (2009) proposed the log-gamma regression model with cure fraction, giving emphasis to the local influence approach.

However, in many situations there are continuous covariates with a nonlinear effect in the response variable and a more flexible or less restrict assumptions are desirable. In the words of Hardle (1990), " . . statisticians realized that pure parametric thinking in curve estimation often does not meet the need for flexibility in data analysis ..." . Therefore, we think that a non-parametric approach in one or more covariates may be a suitable choice to control the effect of continuous covariates, or even to explain nonlinear tendencies of continuous explanatory variables. Several fields have obtained benefits of this perspective, one of the most representative is Economics, at least this is the central message that Yatchew (1998) gives us.

In the context of semi-parametric regression models various works were published in the last decades. For example, Hastie and Tibshirani (1990) developed the theory of generalized additive models, in which non-parametric components are introduced into the systematic component of generalized linear models (McCullagh and Nelder (1989)), whereas Green and Silverman (1994) presented some approach based in natural cubic splines and second derivative penalization. Eilers and Marx (1996) proposed a very flexible approach for semi-parametric regression models, based on P-splines, which consists on the application of the B-splines basis functions derived by De Boor (1978) with a penalization based on the coefficient differences. Rigby and Stasinopoulos (2006) introduced the GAMLSS (Generalized Additive Models for Location, Scale and Shape), which include a wide class of univariate error distributions and one has the possibility of non-parametric components. A review on semi-parametric regression models, based on B-splines, may be found in (Wood, 2006, chapter 4). In particular, under symmetric error models, Ibacache-Pulgar and Paula (2011) derived lo- 
cal influence measures in partially linear Student-t models, extending the work by Kim et al. (2002) that investigated diagnostic methods in partially linear normal models. Relvas and Paula (2016) developed estimation and diagnostic approaches in partially linear models with $\mathrm{AR}(1)$ symmetric errors. IbacachePulgar et al. (2013) discussed an approach for modeling the profiles in elliptical linear mixed models by non-parametric functions. More recently, Vanegas and Paula $(2015,2016)$ discussed the log-symmetric class, that includes various positive continuous asymmetric distributions, and developed estimation procedures for modeling the median and skewness by natural cubic splines, and/or P-splines. A library in R, named ssym Vanegas and Paula (2016), was developed to fit semi-parametric log-symmetric regression models, and in particular it may be applied for fitting symmetric error models.

\subsection{The proposal}

Due to the flexibility of the generalized log-gamma distribution of allowing to the left and to the right-skewed distributions, its potential of application in reliability studies and the few works concerning this distribution in the semiparametric context, we propose to investigate in this project, semi-parametric regression models with generalized log-gamma errors. Thus, our main objectives are:

1. Exploring some properties in the generalized log-gamma class.

2. Understanding the mathematical and statistical background behind the methods of estimation for the semi-parametric models.

3. Studying how to assess the former models through local influence and residual analysis.

4. Developing the $\mathrm{R}$ codes for all the previous methodologies.

5. Studying the statistical properties of the parametric and non-parametric estimators through theoretical developments and simulations.

6. Applying the developed methodologies to real data sets. 


\subsection{The structure}

The project is organized through eight chapters as follows. In chapter 2 we present a review of the generalized log-gamma distribution, adding some new theoretical results. The semi-parametric generalized log-gamma model is introduced in chapter 3. A penalized log-likelihood function is presented as well as the respective score function and Fisher information matrix. This chapter also deals with estimation procedures and inferential results of the semiparametric models, under uncensored observations. In chapter 4 we explore a semi-parametric generalized log-gamma model under independent random censoring. We study how to evaluate the former models through local influence and residual analysis in chapter 5 . We present in chapter 6 the structure and how to use the $\mathbf{R}$ package sglg which was developed to implement the methodologies of this work. Some simulation studies as well as applications to real data sets are given in chapter 7 . We give, in chapter 8 , some conclusions and future path of research. Finally, various technical results are given in the appendices A, B, $\mathrm{C}$ and D. 


\section{Chapter 2}

\section{Generalized log-gamma family}

In this chapter we will give the historical voyage of the generalized log-gamma distribution. Then, we may formulate few questions and try to answer them, at least partially. When was its first appearance? What is its natural context? This family belongs to the location, scale and shape family? Which are its principal subfamilies? Which are its main statistical properties? This family allows multimodal distributions? Each one of the next sections is associated with one or more of the previous questions.

Apart from the previous inquiries, another important goal is to give more detail arguments behind the principal properties of this family, some of them are relatively simple, but other have their complications. In the references we only found some indications or particular cases of some of the properties.

The last important issue is that the results in chapters 3 and 4 have a strong dependency on these properties.

\section{$2.1 \quad$ Some precedents}

Since its first appearance, see for instance, Stacy (1962), the generalized gamma distribution experimented several reparametrizations. According with Hager and Bain (1970) the main reason is that the Newton-Raphson method did not work well and so the existence of solutions to the log-likelihood equations became more complicated. Prentice (1974) proposed a three parameter functional form, in which the normal case is achieved for a finite value of the third parameter and not as a limiting case. The actual form is that proposed by Lawless (1980). The main contexts in which it has been applied are survival analysis and reliability studies. The reason for a wide interest of this distribution is its 
strong relation with distributions such as Weibull, log-normal and exponential. In the last three decades various works related with the log-gamma distribution were developed. For instance, Lawless (1978) built confidence intervals for the parameter of the subfamily of extreme value distributions, DiCiccio (1987) derived approximate inferences for the quantiles and scale parameter, Young and Bakir (1987) obtained the bias of order $n^{-1}$, where $n$ is the sample size, for the parameter estimates in generalized log-gamma regression models without censoring. More recently, Ortega et al. (2008) studied three residuals based on the deviance component in generalized log-gamma regression models under the presence of censored observations.

\subsection{The prototype density}

In this subsection we present a path to obtain the probability density function (p.d.f.) of the generalized log-gamma distribution. This path is slightly different from that given by Prentice (1974), but is completely equivalent. The strategy is to begin from a simple one parametric class of distributions, until obtaining the final form, a three parametric distribution family.

The first step is to consider a random variable $T_{1}$, or the reference distribution, the one-parameter gamma, $\operatorname{Gamma}(k)$, whose probability density function is given by

$$
f_{T_{1}}(t ; k)=\frac{1}{\Gamma(k)} t^{k-1} e^{-t}, t>0, k>0
$$

where $\Gamma(\cdot)$ is the gamma function.

The next step is to consider the random variable, $T_{3}=\log \left(T_{1}\right)$, with probability density function

$$
\begin{aligned}
f_{T_{3}}(t ; k) & =f_{T_{1}}\left(e^{t}\right) e^{t} \\
& =\frac{1}{\Gamma(k)} e^{k t-e^{t}},
\end{aligned}
$$

where, $t \in \mathbb{R}, k>0$,

Now, we build a new random variable, $T_{4}=\frac{T_{3}-\log \left(\lambda^{-2}\right)}{\lambda}$, here we point out that, $\operatorname{Var}\left(T_{3}\right) \approx \frac{1}{k}$ and $k=\lambda^{-2}$. The probability density function of $T_{4}$ has two possible cases: 
If $\lambda>0$

$$
\begin{aligned}
f_{T_{4}}(t ; \lambda) & =f_{T_{3}}\left\{\lambda t+\log \left(\lambda^{-2}\right)\right\} \lambda \\
& =\frac{\lambda}{\Gamma\left(\lambda^{-2}\right)} e^{\lambda^{-2}\left\{\lambda t+\log \left(\lambda^{-2}\right)\right\}-e^{\lambda t+\log \left(\lambda^{-2}\right)}} \\
& =\frac{\lambda}{\Gamma\left(\lambda^{-2}\right)}\left(\lambda^{-2}\right)^{\lambda^{-2}} e^{\frac{t}{\lambda}-\lambda^{-2} e^{\lambda t}} .
\end{aligned}
$$

If $\lambda<0$ and applying a similar calculation we obtain

$$
f_{T_{4}}(t ; \lambda)=\frac{-\lambda}{\Gamma\left(\lambda^{-2}\right)}\left(\lambda^{-2}\right)^{\lambda^{-2}} e^{\frac{t}{\lambda}-\lambda^{-2} e^{\lambda t}} .
$$

Finally, we consider $T_{5}=\mu+\sigma T_{4}$, with $-\infty<\mu<+\infty$ and $\sigma>0$.

Hence, if $\lambda>0$

$$
f_{T_{5}}(t ; \mu, \sigma, \lambda)=\frac{1}{\sigma} \frac{\lambda}{\Gamma\left(\lambda^{-2}\right)}\left(\lambda^{-2}\right)^{\lambda^{-2}} e^{\frac{1}{\lambda \sigma}(t-\mu)-\frac{1}{\lambda^{2}} e^{\frac{\lambda}{\sigma}(t-\mu)}} .
$$

If $\lambda<0$

$$
f_{T_{5}}(t ; \mu, \sigma, \lambda)=\frac{1}{\sigma} \frac{-\lambda}{\Gamma\left(\lambda^{-2}\right)}\left(\lambda^{-2}\right)^{\lambda^{-2}} e^{\frac{1}{\lambda \sigma}(t-\mu)-\frac{1}{\lambda^{2}} e^{\frac{\lambda}{\sigma}(t-\mu)}} .
$$

Remark. There was a random variable $T_{2}=\alpha T_{1}^{1 / \beta}$, which was first proposed by Stacy (1962). The probability density function of $T_{2}$, generalized gamma distribution, called here prototype density, presents complications with the likelihood estimation. This fact was reported by Stacy and Mihram (1965) and Hager and Bain (1970).

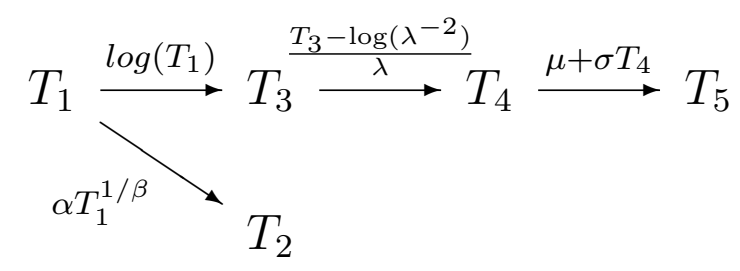

Figure 2.1: A diagrammatic view of the construction process of the $\operatorname{GLG}(\mu, \sigma, \lambda)$. 


\subsection{A tractable density}

The functional form, which we will be considered in this work, is given by the following probability density function:

$$
f(y ; \mu, \sigma, \lambda)=\frac{C(\lambda)}{\sigma} e^{\frac{1}{\lambda \sigma}(y-\mu)-\frac{1}{\lambda^{2}} e^{\frac{\lambda}{\sigma}(y-\mu)}},
$$

where $y \in \mathbb{R}$ and $C(\lambda)=\frac{|\lambda|}{\Gamma\left(\lambda^{-2}\right)}\left(\lambda^{-2}\right)^{\lambda^{-2}}$. The location, scale and shape parameters are $\mu \in \mathbb{R}, \sigma>0$ and $\lambda \in \mathbb{R} \backslash\{0\}$, respectively. When a random variable $Y$ has a p.d.f. given by (2.1), we will denote as $Y \sim \operatorname{GLG}(\mu, \sigma, \lambda)$.

There are two important subfamilies of the generalized log-gamma family, when $\lambda=0$ we have the univariate normal family and when $\lambda=1$ we have the extreme value distribution type I for the minimum and when $\lambda=-1$ we have the extreme value distribution type I for the maximum. In the next section, we will discuss with more detail these cases.

Another appealing feature of this family is if $\lambda<0$, the p.d.f. of $Y$ is to right-skewed and for $\lambda>0$ it is to left-skewed. This kind of behavior will be discussed in section 2.4. As we will show in the next section $\lambda=0$ corresponds to the normal distribution. Figure 2.2 describes the p.d.f. of the GLG $(0,1, \lambda)$ for some $\lambda$ values.

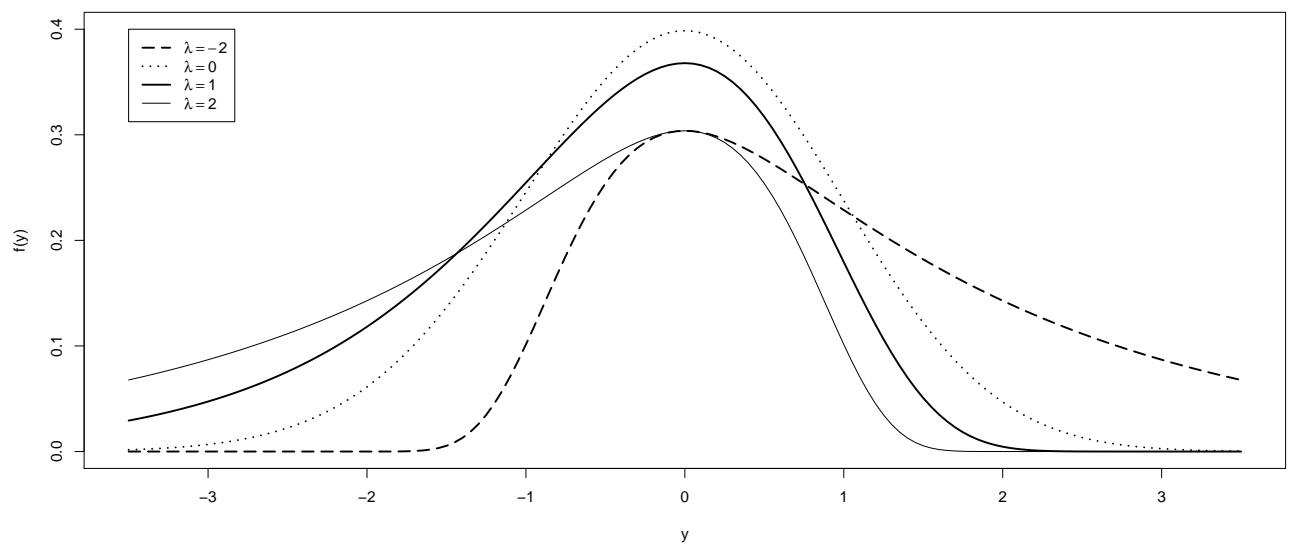

Figure 2.2: Behavior of the p.d.f. of $\operatorname{GLG}(0,1, \lambda)$ for some $\lambda$ values. 
If $\lambda \neq 0$, the expectation and variance of $Y$ are

$$
\mathrm{E}[Y]=\mu+\sigma\left[\frac{\psi\left(\lambda^{-2}\right)-\log \left(\lambda^{-2}\right)}{|\lambda|}\right]
$$

and

$$
\operatorname{Var}[Y]=\sigma^{2} \frac{\psi^{\prime}\left(\lambda^{-2}\right)}{\lambda^{2}}
$$

where $\psi(\cdot)$ is the digamma function and $\psi^{\prime}(\cdot)$ is the trigamma function, which are defined in Appendix B. We will give the proofs for expressions (2.2) and (2.3) in the property 5 of the section 2.4.

\subsubsection{Special cases}

We begin this subsection by discussing on what happens when $\lambda \rightarrow 0$. For convenience, we will express the density (2.1) in terms of $k$. Hence, we want to investigate the behavior of

$$
f(y ; 0,1, k)=\frac{k^{k-\frac{1}{2}}}{\Gamma(k)} e^{\sqrt{k} y-k e^{\frac{y}{\sqrt{k}}}},
$$

when $k \rightarrow \infty$, but $y$ is fixed.

One way to study this limit is to take logarithm in both sides of the equation. So, we obtain

$$
\log \{f(y ; 0,1, k)\}=\left(k-\frac{1}{2}\right) \log (k)-\log \Gamma(k)+\sqrt{k} y-k e^{\frac{y}{\sqrt{k}}} .
$$

In this point we may apply the suggestion in Lawless (1980), that is

$$
\left(k-\frac{1}{2}\right) \log (k)-\log \Gamma(k)=k-\frac{1}{2} \log (2 \pi)-\frac{1}{12 k}+O\left(k^{-3}\right) .
$$

So, we can work with

$$
\lim _{k \rightarrow \infty}\left[k-\frac{1}{2} \log (2 \pi)-\frac{1}{12 k}+\sqrt{k} y-k e^{\frac{y}{\sqrt{k}}}\right] .
$$

But, we know that

$$
e^{\frac{y}{\sqrt{k}}}=1+\frac{y}{\sqrt{k}}+\frac{y^{2}}{2 k}+\frac{y^{3}}{6 \sqrt{k^{3}}} \cdots
$$


Therefore,

$$
k+\sqrt{k} y-k e^{\frac{y}{\sqrt{k}}}=-\frac{y^{2}}{2}-\frac{y^{3}}{6 \sqrt{k}}-\cdots
$$

Thus,

$$
\lim _{k \rightarrow \infty}\left[k-\frac{1}{2} \log (2 \pi)-\frac{1}{12 k}+\sqrt{k} y-k e^{\frac{y}{\sqrt{k}}}\right]=-\frac{1}{2} \log (2 \pi)-\frac{y^{2}}{2} .
$$

Finally, we obtain

$$
\lim _{k \rightarrow \infty} \log \{f(y ; 0,1, k)\}=-\frac{1}{2} \log (2 \pi)-\frac{y^{2}}{2} .
$$

Equivalently,

$$
\lim _{k \rightarrow \infty} f(y ; 0,1, k)=\frac{1}{\sqrt{2 \pi}} e^{-\frac{y^{2}}{2}} .
$$

So, this is the first special case, the normal distribution $N(0,1)$.

Fortunately, our second case is more simple to introduce, it just considers the p.d.f. of the generalized log-gamma distribution when $\lambda=1$, that is,

$$
f(y ; \mu, \sigma)=\frac{1}{\sigma} e^{\frac{1}{\sigma}(y-\mu)-e^{\frac{1}{\sigma}(y-\mu)}} .
$$

This family is known as the Extreme Value Distribution Type I, and denoted by $\operatorname{EV}(\mu, \sigma)$. We will also study this particular family.

\subsection{Statistical properties}

In the following, we will present some useful statistical properties of the generalized log-gamma distribution.

Property 1. Suppose $Y \sim \operatorname{GLG}(\mu, \sigma, \lambda)$, then $\alpha Y \sim \operatorname{GLG}(\alpha \mu,|\alpha| \sigma, \operatorname{sgn}(\alpha) \lambda)$ for all $\alpha \neq 0$.

Proof:

$$
\begin{aligned}
f_{\alpha y}(y) & =\frac{\operatorname{sgn}(\alpha)}{\alpha} f_{y}\left(\frac{y}{\alpha} ; \mu, \sigma, \lambda\right) \\
& =\frac{C(\lambda)}{|\alpha| \sigma} e^{\frac{1}{\lambda \sigma}\left(\frac{y}{\alpha}-\mu\right)-\frac{1}{\lambda^{2}} e^{\frac{\lambda}{\sigma}\left(\frac{y}{\alpha}-\mu\right)}} \\
& =\frac{C(\operatorname{sgn}(\alpha) \lambda)}{|\alpha| \sigma} e^{\frac{1}{\operatorname{sgn}(\alpha) \lambda|\alpha| \sigma}(y-\alpha \mu)-\frac{1}{\lambda^{2}}} e^{\frac{\operatorname{sgn}(\alpha) \lambda}{|\alpha| \sigma}(y-\alpha \mu)}
\end{aligned}
$$


from the definition, $C(\lambda)=C(\operatorname{sgn}(\alpha) \lambda)$.

This means that the multiplication by a constant will produce an effect in all the parameters of the distribution. An important corollary of the previous property is when $\alpha=-1$. It gives us a closure property by the symmetric transformation in this class of distributions.

Corollary 1. Suppose $Y \sim \operatorname{GLG}(\mu, \sigma, \lambda)$ then $-Y \sim \operatorname{GLG}(-\mu, \sigma,-\lambda)$.

The previous corollary generalizes the one given in section 3 of Ferrari and Pinheiro (2012) and allows to establish an equivalence between two types of models. In other words, we may adapt the inference from the models with $\lambda>0$ for models with $\lambda<0$, through the change of $Y$ values and $\mu$ by their symmetric values $-Y$ and $-\mu$, respectively.

The properties 2, 3, and 4 were proposed in sections 3 and 5 of Lawless (1980), but almost without any justification, so we would like to give a more detailed reasoning.

For the next properties for convenience, we will express our density (2.1) in terms of $k$.

Property 2. Suppose $Y \sim \operatorname{GLG}(0,1, k)$, then

$$
\operatorname{Pr}(Y \leq y)=\mathrm{I}\left(k, k e^{\frac{y}{\sqrt{k}}}\right),
$$

where $\mathrm{I}(k, x)=\frac{1}{\Gamma(k)} \int_{0}^{x} v^{k-1} e^{-v} d v$ is the incomplete gamma function.

Proof:

By definition, we know that

$$
\operatorname{Pr}(Y \leq y)=\int_{-\infty}^{y} \frac{k^{k-\frac{1}{2}}}{\Gamma(k)} e^{\sqrt{k} u-k e^{\frac{u}{\sqrt{k}}}} d u .
$$

Changing the variable, $v=k e^{\frac{u}{\sqrt{k}}}$, we obtain

$$
\begin{aligned}
u & =\sqrt{k} \log \left(\frac{v}{k}\right) \\
d u & =\frac{\sqrt{k}}{v} d v .
\end{aligned}
$$


Hence,

$$
\begin{aligned}
e^{\sqrt{k} u-k e^{\frac{u}{\sqrt{k}}}} & =\left(\frac{v}{k}\right)^{k} e^{-v} \\
e^{\sqrt{k} u-k e^{\frac{u}{\sqrt{k}}}} d u & =\frac{v^{k-1}}{k^{k-\frac{1}{2}}} e^{-v} d v \\
\frac{k^{k-\frac{1}{2}}}{\Gamma(k)} e^{\sqrt{k} u-k e^{\frac{u}{\sqrt{k}}}} d u & =\frac{1}{\Gamma(k)} v^{k-1} e^{-v} d v .
\end{aligned}
$$

Then, we obtain

$$
\operatorname{Pr}(Y \leq y)=\int_{0}^{k e^{\frac{y}{\sqrt{k}}}} \frac{1}{\Gamma(k)} v^{k-1} e^{-v} d v
$$

that is exactly we want to prove.

In terms of $\lambda$, we have that

$$
\operatorname{Pr}(Y \leq y)=\mathrm{I}\left(\lambda^{-2}, \lambda^{-2} e^{|\lambda| y}\right)
$$

Remark. The property 2 will be useful for constructing confidence intervals for the parameters as well as to study the survival function associated with the generalized log-gamma distribution.

Property 3. Suppose $Y \sim \operatorname{GLG}(0,1, k)$, then its $p$ th quantile is given by

$$
Q(p, k)=\sqrt{k} \log \left(\frac{1}{2 k} \chi_{2 k, p}^{2}\right)
$$

where $\chi_{2 k, p}^{2}$ denotes the $p$ th quantile of the chi-squared distribution with $2 k$ degrees of freedom. Then, the $p$ th quantile of $Y \sim \operatorname{GLG}(\mu, \sigma, k)$ becomes given by

$$
y_{p, k}=\mu+\sigma Q(p, k)
$$

Proof:

Recall that the expression of the p.d.f. of a variable $\chi_{k}^{2}$ is

$$
f(x ; k)=\frac{1}{\Gamma\left(\frac{k}{2}\right) 2^{\frac{k}{2}}} x^{\frac{k}{2}-1} e^{-\frac{x}{2}} .
$$


So, the p.d.f. of a variable $\chi_{2 k}^{2}$ becomes

$$
f(x ; 2 k)=\frac{1}{\Gamma(k) 2^{k}} x^{k-1} e^{-\frac{x}{2}} .
$$

If we take $W=\frac{1}{2} \chi_{2 k}^{2}$, then

$$
\begin{aligned}
f_{W}(w ; k) & =2 f_{\chi_{2 k}^{2}}(2 w ; 2 k) \\
& =\frac{1}{\Gamma(k)} w^{k-1} e^{-w}
\end{aligned}
$$

Now, the $p$ th quantile of $Y$, denoted by $Q(p, k)$, satisfies

$$
\mathrm{I}\left(k, k e^{\frac{Q(p, k)}{\sqrt{k}}}\right)=p .
$$

From property 2 we know that $V=k e^{\frac{Y}{\sqrt{k}}}$ has the same p.d.f. of $W$. Therefore,

$$
k e^{\frac{Q(p, k)}{\sqrt{k}}}=w_{p}
$$

where $w_{p}$ is the $p$ th quantile of $W$, which is equivalent to the statement of the property.

Property 4. Suppose $Y \sim \operatorname{GLG}(0,1, k)$, then

$$
\begin{aligned}
M_{Y}(t) & =\frac{\Gamma(t \sqrt{k}+k)}{k^{t \sqrt{k}} \Gamma(k)}, \text { if } t>-\sqrt{k} . \\
\mathrm{E}(Y) & =\sqrt{k}(\psi(k)-\log (k)) \text { and } \\
\operatorname{Var}(Y) & =k \psi^{\prime}(k) .
\end{aligned}
$$

\section{Proof:}

By definition,

$$
E\left[e^{t Y}\right]=\int_{-\infty}^{\infty} e^{t y} \frac{k^{k-\frac{1}{2}}}{\Gamma(k)} e^{\sqrt{k} y-k e^{\frac{y}{\sqrt{k}}}} d y .
$$

We can do the same change of variable from property $2, u=k e^{\frac{y}{\sqrt{k}}}$, so we know that

$$
\frac{k^{k-\frac{1}{2}}}{\Gamma(k)} e^{\sqrt{k} y-k e^{\frac{y}{\sqrt{k}}}} d y=\frac{1}{\Gamma(k)} u^{k-1} e^{-u} d u \text {. }
$$


Additionally, we get

$$
e^{t y}=\frac{u^{t \sqrt{k}}}{k^{t \sqrt{k}}}
$$

Therefore,

$$
\begin{aligned}
e^{t y} \frac{k^{k-\frac{1}{2}}}{\Gamma(k)} e^{\sqrt{k} y-k e^{\frac{y}{\sqrt{k}}}} d y & =\frac{u^{t \sqrt{k}}}{k^{t \sqrt{k}}} \frac{1}{\Gamma(k)} u^{k-1} e^{-u} d u \\
& =\frac{u^{t \sqrt{k}+k-1}}{k^{t \sqrt{k}}} \frac{1}{\Gamma(k)} e^{-u} d u .
\end{aligned}
$$

Thus,

$$
E\left[e^{t Y}\right]=\frac{1}{k^{t \sqrt{k}} \Gamma(k)} \int_{0}^{\infty} u^{t \sqrt{k}+k-1} e^{-u} d u,
$$

that is just the statement of the property, if $t>-\sqrt{k}$. Now, we continue with the expectation of $Y$, by taking

$$
M_{Y}^{\prime}(t)=\frac{\sqrt{k} \Gamma^{\prime}(t \sqrt{k}+k) \Gamma(k) k^{t \sqrt{k}}-\Gamma(t \sqrt{k}+k) \Gamma(k)\left(k^{t \sqrt{k}}\right)^{\prime}}{k^{2 t \sqrt{k}} \Gamma^{2}(k)} .
$$

But,

$$
\left(k^{t \sqrt{k}}\right)^{\prime}=\sqrt{k} \log (\mathrm{k}) k^{t \sqrt{k}}
$$

SO,

$$
M_{Y}^{\prime}(t)=\frac{\sqrt{k}}{\Gamma(k)}\left[\frac{\Gamma^{\prime}(t \sqrt{k}+k)-\Gamma(t \sqrt{k}+k) \log (\mathrm{k})}{k^{t \sqrt{k}}}\right] .
$$

Therefore,

$$
M_{Y}^{\prime}(0)=\sqrt{k}\left[\frac{\Gamma^{\prime}(k)}{\Gamma(k)}-\log (k)\right]
$$

which is exactly we want to prove, because $\psi(k)=\frac{\Gamma^{\prime}(k)}{\Gamma(k)}$.

Finally, we find the expression of the variance of $Y$.

It's clear that,

$$
\left[M_{Y}^{\prime}(0)\right]^{2}=\frac{k}{\Gamma^{2}(k)}\left[\left(\Gamma^{\prime}(k)\right)^{2}-2 \Gamma(k) \Gamma^{\prime}(k) \log (k)+\Gamma^{2}(k) \log ^{2}(k)\right] .
$$


Also, we have

$$
M_{Y}^{\prime \prime}(t)=\frac{k}{\Gamma(k)}\left[\frac{\Gamma^{\prime \prime}(t \sqrt{k}+k)-2 \Gamma^{\prime}(t \sqrt{k}+k) \log (k)+\Gamma(t \sqrt{k}+k) \log ^{2}(k)}{k^{t \sqrt{k}}}\right]
$$

and

$$
M_{Y}^{\prime \prime}(0)=\frac{k}{\Gamma(k)}\left[\Gamma^{\prime \prime}(k)-2 \Gamma^{\prime}(k) \log (k)+\Gamma(k) \log ^{2}(k)\right] .
$$

Therefore,

$$
\begin{aligned}
M_{Y}^{\prime \prime}(0)-\left[M_{Y}^{\prime}(0)\right]^{2} & =\frac{k}{\Gamma^{2}(k)}\left[\Gamma^{\prime \prime}(k) \Gamma(k)-\left[\Gamma^{\prime}(k)\right]^{2}\right], \\
& =k \psi^{\prime}(k),
\end{aligned}
$$

the equality we want to prove.

Remark. We may also calculate the skewness and kurtosis with the same kind of reasoning from the definition of these quantities. But, we would like to give another approach. Pinhero (2013) calculates the skewness and kurtosis of an exponential-gamma three parametric distribution with aid of a symbolic computational software. The expressions for the skewness and kurtosis are

$$
\gamma_{2}(\lambda)=\operatorname{sign}(\lambda) \frac{\psi^{\prime \prime}\left(1 / \lambda^{2}\right)}{\left\{\psi^{\prime}\left(1 / \lambda^{2}\right)\right\}^{\frac{3}{2}}}
$$

and

$$
\gamma_{3}(\lambda)=\frac{\psi^{\prime \prime \prime}\left(1 / \lambda^{2}\right)}{\left\{\psi^{\prime}\left(1 / \lambda^{2}\right)\right\}^{2}}+3
$$

respectively. The point is that we may consider an exponential-gamma three parametric distribution as an affine transformation of a generalized log-gamma distribution. And, we know that the skewness and kurtosis are invariant under this kind of transformations, so we have achieved the objective.

There are two important consequences from the last property.

Property 5. Suppose $Y \sim \operatorname{GLG}(0,1, \lambda)$, with $\lambda \neq 0$, then

$$
E\left[Y e^{\lambda Y}\right]=\frac{1}{\lambda}\left[\psi\left(1+\frac{1}{\lambda^{2}}\right)-\log \left(\frac{1}{\lambda^{2}}\right)\right]
$$


and

$$
E\left[Y^{2} e^{\lambda Y}\right]=\frac{1}{\lambda^{2}}\left[\psi^{\prime}\left(1+\frac{1}{\lambda^{2}}\right)+\left[\psi\left(1+\frac{1}{\lambda^{2}}\right)-\log \left(\frac{1}{\lambda^{2}}\right)\right]^{2}\right]
$$

Proof:

From the property 4 we know that

$$
\begin{aligned}
& M_{Y}^{\prime}(t)=\frac{\sqrt{k}}{\Gamma(k)}\left[\frac{\Gamma^{\prime}(t \sqrt{k}+k)-\Gamma(t \sqrt{k}+k) \log (\mathrm{k})}{k^{t \sqrt{k}}}\right] \\
& M_{Y}^{\prime \prime}(t)=\frac{k}{\Gamma(k)}\left[\frac{\Gamma^{\prime \prime}(t \sqrt{k}+k)-2 \Gamma^{\prime}(t \sqrt{k}+k) \log (k)+\Gamma(t \sqrt{k}+k) \log ^{2}(k)}{k^{t \sqrt{k}}}\right]
\end{aligned}
$$

But, $E\left[Y e^{\lambda Y}\right]=M_{Y}^{\prime}(\lambda)$ and $E\left[Y^{2} e^{\lambda Y}\right]=M_{Y}^{\prime \prime}(\lambda)$ which implies the expressions we want.

Remark. These quantities will appear to evaluate the Fisher information matrix in the iterative process for the parameter estimation. They will reveal essential for efficiency of the algorithms of glg and sglg, chapter 7. A few words with respect to the special functions that appeared in this property. According with Casella and Berger (2002), "the estimation of the mean of a gamma distribution is not an easy task ... we have to deal with the digamma function, which is never pleasant."

Property 6. The generalized log-gamma family is a unimodal family. Let $\operatorname{Mo}(\mu, \sigma, \lambda)$ denote the mode of $\operatorname{GLG}(\mu, \sigma, \lambda)$. Then,

$$
\operatorname{Mo}(\mu, \sigma, \lambda)=\mu \text {. }
$$

\section{Proof:}

From (2.1) it is clear that $f(y ; \mu, \sigma, \lambda)$ is a differentiable function with respect to $y$ in $(-\infty, \infty)$. The idea for proving the unimodality of the family is that there is only one singular point which would be the maximum of the probability density function.

Now, let $\lambda \neq 0$, the expression for $f^{\prime}(y ; \mu, \sigma, \lambda)$ is

$$
\begin{aligned}
f^{\prime}(y ; \mu, \sigma, \lambda) & =\frac{C(\lambda)}{\sigma}\left[\left\{\frac{1}{\lambda \sigma}(y-\mu)-\frac{1}{\lambda^{2}} e^{\frac{\lambda}{\sigma}(y-\mu)}\right\}^{\prime} e^{\left.\frac{1}{\lambda \sigma}(y-\mu)-\frac{1}{\lambda^{2}} e^{\frac{\lambda}{\sigma}(y-\mu)}\right]}\right. \\
& =\frac{C(\lambda)}{\sigma}\left[\left\{\frac{1}{\lambda \sigma}-\frac{1}{\lambda \sigma} e^{\frac{\lambda}{\sigma}(y-\mu)}\right\} e^{\frac{1}{\lambda \sigma}(y-\mu)-\frac{1}{\lambda^{2}} e^{\frac{\lambda}{\sigma}(y-\mu)}}\right] .
\end{aligned}
$$


The only way that the previous expression equals to zero is when

$$
\frac{1}{\lambda \sigma}-\frac{1}{\lambda \sigma} e^{\frac{\lambda}{\sigma}(y-\mu)}=0 .
$$

So, this condition leads to $y=\mu$. For $\lambda=0$ we have known that is the normal case.

Let $Y \sim \operatorname{GLG}(\mu, \sigma, \lambda)$, we will denote its median by $\operatorname{Md}(\mu, \sigma, \lambda)$.

Property 7. Let $Y \sim \operatorname{GLG}(\mu, \sigma, \lambda)$,

if $\lambda>0$, we have

$$
\begin{gathered}
\operatorname{Md}(\mu, \sigma, \lambda)<\mu \text { and } \\
E[Y]<\mu .
\end{gathered}
$$

if $\lambda<0$, we have

$$
\begin{aligned}
\operatorname{Md}(\mu, \sigma, \lambda) & >\mu \text { and } \\
E[Y] & >\mu .
\end{aligned}
$$

Proof:

From Property 2, we know that

$$
\operatorname{Pr}(Y \leq y)=I\left(\lambda^{-2}, \lambda^{-2} e^{|\lambda| \frac{y-\mu}{\sigma}}\right) .
$$

So, if we take $y=\mu$, we obtain

$$
\operatorname{Pr}(Y \leq \mu)=I\left(\lambda^{-2}, \lambda^{-2}\right) .
$$

This quantity is always greater than 0.5 by the following inequality:

$$
z-e^{z}<-z-e^{-z} \text { for } z>0,
$$

which is the base of the Theorem 1 of Chen and Rubin (1986), and means that this statement is true.

The other inequality of this property becomes from the next fact

$$
\begin{aligned}
& \psi(x) \leq \log (x) \text { for } x>0 \\
& \lim _{x \rightarrow \infty} \frac{\psi(x)}{\log (\mathrm{x})}=1 .
\end{aligned}
$$


So, we have that for $\lambda>0$

$$
\begin{aligned}
\sigma\left[\frac{\psi\left(\lambda^{-2}\right)-\log \left(\lambda^{-2}\right)}{|\lambda|}\right] & \leq 0 \text { and } \\
\mu+\sigma\left[\frac{\psi\left(\lambda^{-2}\right)-\log \left(\lambda^{-2}\right)}{|\lambda|}\right] & \leq \mu .
\end{aligned}
$$

If we have that for $\lambda<0$

$$
\begin{aligned}
\sigma\left[\frac{\psi\left(\lambda^{-2}\right)-\log \left(\lambda^{-2}\right)}{|\lambda|}\right] & \geq 0 \text { and } \\
\mu+\sigma\left[\frac{\psi\left(\lambda^{-2}\right)-\log \left(\lambda^{-2}\right)}{|\lambda|}\right] & \geq \mu .
\end{aligned}
$$

We achieve the equality only when $\lambda=0$.

The next property, derived in this work, is the entropy of the generalized log-gamma distribution. According with Balakrishnan and Nevzorov (2003) entropy is one of the more useful characteristics of a distribution. If $Y$ has a continuous distribution with p.d.f. $f(y)$, then the entropy is defined as

$$
H(Y)=-\int_{D} f(y) \log (f(y)) d y
$$

where

$$
D=\{y: f(y)>0\}
$$

The behavior of the entropy under transformation is given as follows, if $Z=$ $\mu+\sigma Y$, with $\sigma>0$, then

$$
H(Z)=\log (\sigma)+H(Y)
$$

From the previous relation, we will derive the entropy of $Y \sim \operatorname{GLG}(0,1, \lambda)$, and the general case will be an immediate consequence.

Property 8. Let $Y \sim \operatorname{GLG}(0,1, \lambda)$, and $\lambda \neq 0$, then

$$
H(Y)=\log \left[\frac{\Gamma\left(\frac{1}{\lambda^{2}}\right)}{|\lambda|}\right]+\frac{1}{\lambda^{2}}\left[1-\psi\left(\frac{1}{\lambda^{2}}\right)\right] .
$$

\section{Proof:}

The p.d.f. of $Y \sim \operatorname{GLG}(0,1, \lambda)$ is

$$
f_{Y}(y ; \lambda)=C(\lambda) e^{\frac{y}{\lambda}-\frac{1}{\lambda^{2}} e^{\lambda y}} .
$$


So,

$$
-\log \left(f_{Y}(Y ; \lambda)\right)=-\log (C(\lambda))-\frac{1}{\lambda} Y+\frac{1}{\lambda^{2}} e^{\lambda Y} .
$$

Therefore,

$$
H(Y)=-\log (C(\lambda))-\frac{1}{\lambda} E[Y]+\frac{1}{\lambda^{2}} M_{Y}(\lambda) .
$$

Now, by definition, $C(\lambda)=\frac{|\lambda|}{\Gamma\left(\lambda^{-2}\right)}\left(\lambda^{-2}\right)^{\lambda^{-2}}$, then

$$
-\log (C(\lambda))=-\log (|\lambda|)-\frac{1}{\lambda^{2}} \log \left(\frac{1}{\lambda^{2}}\right)+\log \left(\Gamma\left(\frac{1}{\lambda^{2}}\right)\right) .
$$

By other hand,

$$
E[Y]=\frac{1}{\lambda}\left[\psi\left(\frac{1}{\lambda^{2}}\right)-\log \left(\frac{1}{\lambda^{2}}\right)\right] .
$$

We know that $k=\frac{1}{\lambda^{2}}$ combined with property 5 leads to

$$
M_{Y}(t)=\frac{\Gamma\left(\frac{t}{\lambda}+\frac{1}{\lambda^{2}}\right)}{\left(\frac{1}{\lambda^{2}}\right)^{\frac{t}{\lambda}} \Gamma\left(\frac{1}{\lambda^{2}}\right)} .
$$

Hence,

$$
M_{Y}(\lambda)=1 .
$$

With all the previous ingredients we get the expression.

\subsubsection{A glimpse to the lifetime analysis}

When we have a non-negative random variable $T$, representing the lifetimes of individuals, with cumulative distribution function $F(t)$, then we can define a series of functions associated with the random variable $T$. The first one give us the probability of an individual surviving to time $t$, the survivor function $S(t)$

$$
\begin{aligned}
S(t) & =P(T \geq t) \\
& =1-F(t) .
\end{aligned}
$$

The second one, an important concept in lifetime distributions, is the hazard function defined by 


$$
\begin{aligned}
h(t) & =\lim _{\Delta t \rightarrow 0} \frac{P(t \leq T<t+\Delta t \mid T \geq t)}{\Delta t} \\
& =\frac{f(t)}{S(t)} .
\end{aligned}
$$

This function shows the instantaneous rate of failure at time $t$, given that the individual survives up to $t$. More details, see, for instance, Lawless (2003).

Property 9. Let $Y \sim \operatorname{GLG}(\mu, \sigma, \lambda)$, then $T=e^{Y}$ has probability density function

$$
f_{T}(t ; \mu, \sigma, \lambda)=\frac{|\lambda|}{\sigma t \Gamma\left(\lambda^{-2}\right)}\left(\lambda^{-2}\right)^{\lambda^{-2}}\left(e^{-\mu} t\right)^{\frac{1}{\sigma \lambda}} e^{-\left(\lambda^{-2}\right)\left(e^{-\mu} t\right)^{\frac{\lambda}{\sigma}}} .
$$

This distribution is called the generalized gamma distribution, which we will denote as $T \sim \operatorname{GG}(\mu, \sigma, \lambda)$. For a recent study of the hazard functions of this family of distributions, see Cox et al. (2007). There, we can see that the shapes of hazard functions of this distribution cover four of the most common types of hazard functions in practice: increasing, decreasing, bathtub and arc-shaped; which shows the great applicability of this family of probability distributions to survival analysis, see Figure 2.3.

One important corollary of the property 9 is the following:

Corollary 2. Suppose $Y \sim \operatorname{GLG}(\mu, \sigma, \lambda)$

- if $\lambda=1$ and $\sigma=1, e^{Y} \sim \operatorname{Exp}(\mu)$,

- if $\lambda=1, e^{Y} \sim \operatorname{Weibull}(\mu, \lambda)$,

- if $\sigma=\lambda, e^{Y} \sim \operatorname{Gamma}(\mu, \lambda)$,

- if $\lambda=0, e^{Y} \sim \log \operatorname{Normal}(\mu, \lambda)$,

- if $\lambda=-1, e^{Y} \sim$ Inverse $\operatorname{Weibull}(\mu, \lambda)$,

- if $\lambda=-\sigma, e^{Y} \sim$ Inverse gamma $(\mu, \lambda)$,

- if $\lambda=\frac{1}{\sigma}, e^{Y} \sim \operatorname{Ammag}(\mu, \lambda)$ and

- if $\lambda=-\frac{1}{\sigma}, e^{Y} \sim$ Inverse $\operatorname{Ammag}(\mu, \lambda)$. 
Remark. Property 9 and its corollary is key to understand why the generalized log-gamma distribution appears in survival and reliability analysis. The first basic reason is that sometimes is more convenient to work with the logarithm of the lifetimes than with the original values, at least in a computational sense. The second powerful reason is the huge amount of distributions that the generalized gamma covers. For example, Crooks (2015) found 17 particular cases of this distribution. The Ammag and Inverse Ammag distributions are not in the Crook's list but we may find them in Cox et al. (2007).

Now, we define the survivor, hazard functions associated with T, which we may also found in Cox et al. (2007).

Property 10. Suppose $Y \sim \operatorname{GLG}(\mu, \sigma, \lambda)$ and define $T=e^{Y}$, then

$$
\begin{gathered}
S_{G G}= \begin{cases}1-\Gamma\left(\lambda^{-2}\left(t e^{-\mu}\right)^{\frac{\lambda}{\sigma}}, \lambda^{-2}\right) & \text { if } \lambda>0, \\
\Gamma\left(\lambda^{-2}\left(t e^{-\mu}\right)^{\frac{\lambda}{\sigma}}, \lambda^{-2}\right) & \text { if } \lambda<0,\end{cases} \\
h(t ; \lambda)= \begin{cases}\frac{\lambda\left(\lambda^{-2}\right)^{\lambda^{-2}} t^{\frac{1}{\lambda}-1} e^{-\frac{1}{\lambda^{2}} t^{\lambda}}}{\int_{\frac{1}{\lambda^{2}} t^{\lambda^{2}} v^{\lambda^{-2}-1} e^{-v} d v}} & \text { if } \lambda>0, \\
\frac{-\lambda\left(\lambda^{-2}\right)^{\lambda^{-2}} t^{\frac{1}{\lambda}-1} e^{-\frac{1}{\lambda^{2}} t^{\lambda}}}{\int_{0}^{\frac{1}{\lambda^{2}} t^{\lambda}} v^{\lambda^{-2}-1} e^{-v} d v} & \text { if } \lambda<0 .\end{cases}
\end{gathered}
$$

Proof:

See Cox et al. (2007).

\subsubsection{Final remark}

As a curious thing, we would say that Consul and Jain (1971) found expressions for the product and quotient of two independent "log-gamma variables ", but these variables are not log-gamma variables in the same way we are working with. They worked with variables of the type $Z=e^{\frac{T}{v}}$, where $v$ is a parameter and $\mathrm{T}$ is a variable with the distribution proposed by Stacy (1962). So, they called it with the same logic that one variable is called log-normal and clearly is not the case of (2.1). 

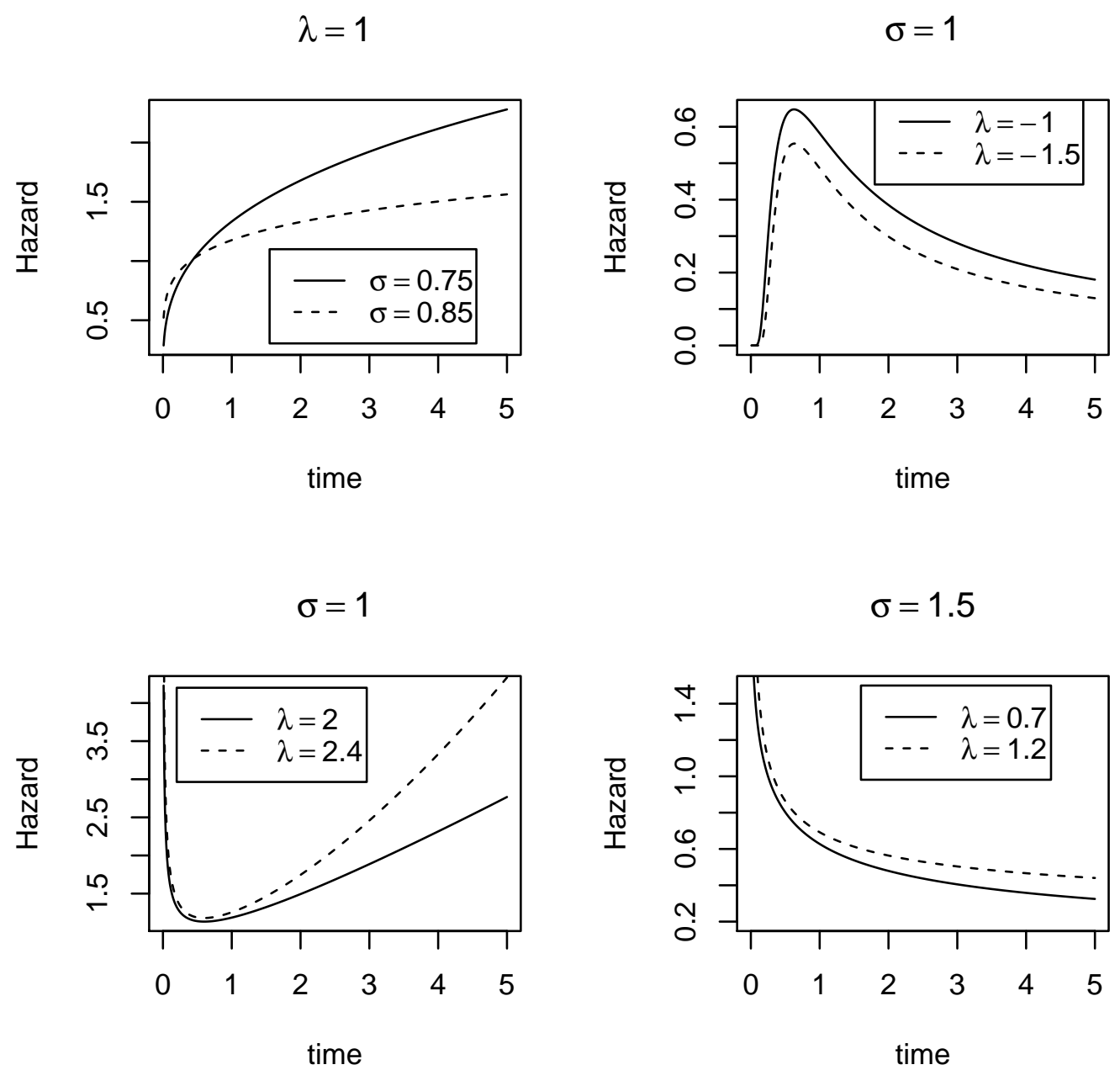

Figure 2.3: Behavior of the hazard function of $\operatorname{GLG}(0, \sigma, \lambda)$ for some values of $(\sigma, \lambda)$. 


\section{Chapter 3}

\section{Inference procedures: uncensored case}

\subsection{Introduction}

The aim of this chapter is to discuss the extension of the theory of semiparametric models to the generalized log-gamma family, under the uncensored case. There is a vast literature on semi-parametric models. For instance, Hastie and Tibshirani (1990) proposed the generalized additive models, whereas Green and Silverman (1994) discussed semi-parametric linear models in the context of the exponential family of distributions under the natural cubic spline approach. Eilers and Marx (1996) proposed the P-spline approach, in which the nonparametric function may be approximated by the B-spline basis functions derived by De Boor (1978), with the penalization being performed by coefficient differences. Rigby and Stasinopoulos (2006) proposed the GAMLSS (Generalized Additive Models for Location, Scale and Shape) and partially linear models may be derived for a wide class of univariate distributions. In Wood (2006) there is a review of semi-parametric models under B-spline approach with various applications to real data sets. Ibacache-Pulgar and Paula (2011) extended the work by Kim et al. (2002) on diagnostic methods in partially linear normal models to the Student-t class, Vanegas and Paula (2016) discussed semi-parametric models under log-symmetric distributions, whereas Relvas and Paula (2016) developed an iterative process as well as diagnostic procedures in partially linear models with first order auto-regressive symmetric errors. More recently, Ferreira and Paula (2017) developed estimation and diagnostic procedures in skew-normal partially linear models. 


\subsection{Partially linear generalized log-gamma re- gression models}

\subsubsection{The model}

Let $y_{1}, \cdots, y_{n}$ be a set of random variable values and we will assume the following relation between the response values and the explanatory variable values:

$$
y_{i}=\mathbf{x}_{i}^{\top} \boldsymbol{\beta}+g\left(t_{i}\right)+\sigma \epsilon_{i},
$$

for $i=1, \cdots, n$, where $\mathbf{x}_{i}=\left(x_{i 1}, \cdots, x_{i p}\right)^{\top}$ is a vector of explanatory variable values, $t_{i}$ is an observed value of a continuous covariate, $\boldsymbol{\beta}=\left(\beta_{1}, \cdots, \beta_{p}\right)^{\top}$ is a $(p \times 1)$ vector of fixed parameters, $g(\cdot)$ is an unknown smoothing function, $\sigma>0$ and $\epsilon_{i}$ 's are independent identically distributed random errors that follow $\operatorname{GLG}(0,1, \lambda)$. The matrix $\mathbf{X}=\left[\left(\mathbf{x}_{1}^{\top}, t_{1}\right), \cdots,\left(\mathbf{x}_{n}^{\top}, t_{n}\right)\right]^{\top}$ is assumed to be full column rank. So, $Y_{i}$ has the distribution

$$
Y_{i} \sim \operatorname{GLG}\left(\mathbf{x}_{i}^{\top} \boldsymbol{\beta}+g\left(t_{i}\right), \sigma, \lambda\right)
$$

with

$$
\mathrm{E}\left[Y_{i}\right]=\mathbf{x}_{i}^{\top} \boldsymbol{\beta}+g\left(t_{i}\right)+\sigma\left[\frac{\psi\left(\lambda^{-2}\right)-\log \left(\lambda^{-2}\right)}{|\lambda|}\right]
$$

and

$$
\operatorname{Var}\left[Y_{i}\right]=\sigma^{2} \frac{\psi^{\prime}\left(\lambda^{-2}\right)}{\lambda^{2}}, \text { for } i=1, \cdots, n
$$

where $\psi(\cdot)$ is the digamma function and $\psi^{\prime}(\cdot)$ is the trigamma function, which are defined in Appendix B.

For estimating the non-parametric functions in the model (3.1) we need a way to represent it or at least approximate it. In the next section we will give two ways to do it.

\subsubsection{Approximating smooth functions}

We are working with two ways of approximating smooth functions, natural cubic splines and P-splines. 


\section{Natural cubic spline}

The first step in the natural cubic spline approach of the smooth component $g(\cdot)$ is to identify and ordering the distinct values of $t_{i}(i=1, \cdots, n)$. Second step is to select the number of knots $q$ that we will employ. The third step is to select the set of knots $\left\{k_{1}, \cdots, k_{q}\right\}$, such that, $k_{1}=\min (\mathbf{t}), k_{q}=\max (\mathbf{t})$ and $\mathbf{t}=\left\{t_{1}, \cdots, t_{n}\right\}$. So, we have

$$
g\left(t_{i}\right)=\sum_{j=1}^{q} N_{i j} \gamma_{j},
$$

where $N_{i j}$ is the $(i, j)$-th component of the basis matrix which depends on the natural spline cubic basis. In this work we implement the basis of $\mathrm{Gu}$ and Kim (2002) and $\gamma_{j}$ is the coefficient of $g(\cdot)$ with respect to the element of the basis at the knot $k_{j}$. Finally, we can calculate the natural cubic spline approximation of $g(t)$ at the point $k_{1} \leq t \leq k_{q}$ by the general form

$$
g(t)=\sum_{j=1}^{q} N_{j}(t) \gamma_{j},
$$

where $N_{j}(t)$ denotes the $j$ th basis component evaluated at the value $k_{1} \leq t \leq k_{q}$.

\section{P-splines}

The first two steps in the P-spline approach are the same that in the cubic spline approach. First, to identify and to order the distinct values of $t_{i}(i=$ $1, \cdots, n)$. Second, to select the number of internal knots $q$. But, unlike the natural cubic spline approach, we need to add $2 * o$ knots, such that, $k_{1+o}=$ $\min (\mathbf{t})$ and $k_{q+o}=\max (\mathbf{t})$, where $o$ is the order of the elements of the B-spline basis. So, we have

$$
g(t)=\sum_{j=1}^{q} B_{j}(t, o-1) \gamma_{j}
$$

approximately, where $B_{j}(\cdot, o-1)$ is the $j$ th element of order $o$ of the B-spline basis that we had chosen and $\gamma_{j}$ is the coefficient of $g(t)$ with respect to this basis.

For both approaches, in matrix notation we have

$$
\mathbf{y}=\mathbf{X} \boldsymbol{\beta}+\mathbf{N} \boldsymbol{\gamma}+\boldsymbol{\epsilon},
$$


where $\mathbf{y}=\left(y_{1}, \cdots, y_{n}\right)^{\top}, \mathbf{X}$ is an $(n \times p)$ matrix with rows given by $\mathbf{x}_{i}^{\top}, \mathbf{N}$ is a $(n \times q)$ basis matrix, $\gamma=\left(\gamma_{1}, \cdots, \gamma_{q}\right)^{\top}$ is a $(q \times 1)$ vector of parameters associated with $g(\cdot)$ and $\boldsymbol{\epsilon}=\left(\epsilon_{1}, \cdots, \epsilon_{n}\right)^{\top}$ is a vector of errors. Now, we have a form to represent the non-parametric component. But, how should we to estimate parameters present in "the new form" of the model (3.1)? The next subsection introduce a key concept which allow us to do the estimating process: the penalized log-likelihood function.

For more details on natural cubic splines and P-splines approaches as well as the basis we had worked with, see Appendix A.

\subsubsection{Penalized log-likelihood function}

The joint density function of a random sample $y_{1}, \cdots, y_{n}$ may be expressed as $f\left(y_{1}, \cdots, y_{n} ; \boldsymbol{\theta}\right)$. The associated $\log$-likelihood function takes the form

$$
L(\boldsymbol{\theta})=\log \left\{f\left(y_{1}, \cdots, y_{n} ; \boldsymbol{\theta}\right)\right\}=n \log \left(\frac{C(\lambda)}{\sigma}\right)+\frac{1}{\lambda} \sum_{i=1}^{n} \epsilon_{i}-\frac{1}{\lambda^{2}} \sum_{i=1}^{n} e^{\lambda \epsilon_{i}},
$$

where $\boldsymbol{\theta}=\left(\boldsymbol{\beta}^{\top}, \boldsymbol{\gamma}^{\top}, \sigma, \lambda\right)^{\top}, \epsilon_{i}=\frac{y_{i}-\mathbf{x}_{i}^{\top} \boldsymbol{\beta}-\mathbf{N}_{i} \boldsymbol{\gamma}}{\sigma}$, and $\mathbf{x}_{i}^{\top}$ and $\mathbf{N}_{i}$ are rows of the matrices $\mathbf{X}$ and $\mathbf{N}$, respectively. The maximization of (3.4) without any restriction over the non-parametric function $g(\cdot)$ may cause overfitting. One possible solution to the overfitting is to incorporate a penalty over $g(\cdot)$. If we employ a natural cubic spline approximation, Green and Silverman (1994) proposed the following penalty:

$$
\frac{\alpha}{2} \int_{a}^{b}\left[g^{\prime \prime}(t)\right]^{2} d t
$$

If $g(\cdot)$ is represented by P-splines, Eilers and Marx (1996) proposed to apply the following difference penalty of order $v$ over adjacent knots:

$$
\frac{\alpha}{2} \sum_{j=1}^{q}\left[\Delta^{v} \gamma_{j}\right]^{2}
$$

where $\Delta^{v} \gamma_{j}=\sum_{m=1}^{v}(-1)^{v}\left(\begin{array}{l}v \\ m\end{array}\right) \gamma_{j-m}$, we will use $v=2$.

The previous conditions are incorporated into the log-likelihood function as

$$
L_{p}(\boldsymbol{\theta}, \alpha)=n \log \left(\frac{C(\lambda)}{\sigma}\right)+\frac{1}{\lambda} \sum_{i=1}^{n} \epsilon_{i}-\frac{1}{\lambda^{2}} \sum_{i=1}^{n} e^{\lambda \epsilon_{i}}-\frac{\alpha}{2} \boldsymbol{\gamma}^{\top} \mathbf{M} \boldsymbol{\gamma},
$$


where $\mathbf{M}$ is a semi-positive defined matrix which depends on the natural cubic spline basis or B-spline basis we have chosen to work with.

Moreover, the smoothing parameter, $\alpha>0$, control the tradeoff between goodness-of-fit and the smoothness of the estimated function. There are various methods for the determination of $\alpha$, such as optimization of the generalized cross-validation, AIC or SIC criteria, which will be discussed, respectively, in the next sections. In particular, for natural cubic splines, when $\alpha \rightarrow 0$ the fit of the non-parametric component $g(t)$ tends to a data interpolation, whereas when $\alpha \rightarrow \infty$ we have to impose $g^{\prime \prime}(t)=0$ that implies $g(t)$ be a linear function.

\subsection{Parameter estimation}

One possibility to estimate the parameters is to apply the Newton-Raphson method. Other option is to use a methodology based on the Fisher scoring method and back-fitting algorithm for maximizing the penalized log-likelihood function. In order to perform an iterative procedure for the estimation of $\boldsymbol{\beta}, \boldsymbol{\gamma}, \sigma, \lambda$, we need to derive the penalized score function and the penalized Fisher information matrix, which are derived in Appendix B.

\subsubsection{Penalized score function and penalized Fisher in- formation matrix}

The penalized score function for $\boldsymbol{\theta}$ may be expressed as

$$
\mathbf{U}_{p}^{\boldsymbol{\theta}}=\left[\begin{array}{c}
\mathbf{U}_{p}^{\beta} \\
\mathbf{U}_{p}^{\gamma} \\
U_{p}^{\sigma} \\
U_{p}^{\lambda}
\end{array}\right]=\left[\begin{array}{c}
-\frac{1}{\lambda \sigma} \mathbf{X}^{\top}\{\mathbf{I}-\mathbf{D}(\mathbf{d})\} \mathbf{1} \\
-\frac{1}{\lambda \sigma} \mathbf{N}^{\top}\{\mathbf{I}-\mathbf{D}(\mathbf{d})\} \mathbf{1}-\alpha \mathbf{M} \boldsymbol{\gamma} \\
-\frac{1}{\sigma} \mathbf{1}^{\top} \mathbf{1}-\frac{1}{\lambda \sigma} \mathbf{1}^{\top}\{\mathbf{I}-\mathbf{D}(\mathbf{d})\} \boldsymbol{\epsilon} \\
n \zeta_{\lambda}-\frac{1}{\lambda^{2}} \mathbf{1}^{\top} \boldsymbol{\epsilon}+\frac{2}{\lambda^{3}} \mathbf{1}^{\top} \mathbf{D}(\mathbf{d}) \mathbf{1}-\frac{1}{\lambda^{2}} \mathbf{1}^{\top} \mathbf{D}(\mathbf{d}) \boldsymbol{\epsilon}
\end{array}\right]
$$

where $\zeta_{\lambda}=\frac{1}{\lambda}+\frac{2}{\lambda^{3}}\left\{\psi\left(\frac{1}{\lambda^{2}}\right)+2 \log (|\lambda|)-1\right\}, \mathbf{D}(\mathbf{d})$ is a $(n \times n)$ diagonal matrix, $\mathbf{d}=\left(d_{1}, \cdots, d_{n}\right)$ with $d_{i}=e^{\lambda \epsilon_{i}}, \epsilon_{i}=\frac{y_{i}-\mathbf{x}_{i}^{\top} \boldsymbol{\beta}-\mathbf{N}_{i} \boldsymbol{\gamma}}{\sigma}, \mathbf{1}$ is an $(n \times 1)$ vector of ones and $\mathbf{I}$ denotes the identity matrix of order $n$. 
The penalized Fisher information matrix for $\boldsymbol{\theta}$ assumes the form

$$
\mathbf{I}_{p}^{\boldsymbol{\theta \theta}}=\left[\begin{array}{cccc}
\frac{r_{\lambda}}{\sigma^{2}} \mathbf{X}^{\top} \mathbf{X} & \frac{r_{\lambda}}{\sigma^{2}} \mathbf{X}^{\top} \mathbf{N} & \frac{1}{\lambda \sigma^{2}} \Psi_{\lambda} \mathbf{X}^{\top} \mathbf{1} & \frac{1}{\lambda^{2} \sigma} \kappa_{3, \lambda} \mathbf{X}^{\top} \mathbf{1} \\
\frac{r_{\lambda}}{\sigma^{2}} \mathbf{N}^{\top} \mathbf{X} & \frac{r_{\lambda}}{\sigma^{2}} \mathbf{N}^{\top} \mathbf{N}+\alpha \mathbf{M} & \frac{1}{\lambda \sigma^{2}} \Psi_{\lambda} \mathbf{N}^{\top} \mathbf{1} & \frac{1}{\lambda^{2} \sigma} \kappa_{3, \lambda} \mathbf{N}^{\top} \mathbf{1} \\
\frac{1}{\lambda \sigma^{2}} \Psi_{\lambda} \mathbf{1}^{\top} \mathbf{X} & \frac{1}{\lambda \sigma^{2}} \Psi_{\lambda} \mathbf{1}^{\top} \mathbf{N} & \frac{n}{\sigma^{2}}\left(1+v_{\lambda}\right) & \frac{n}{\lambda^{2} \sigma} \kappa_{2, \lambda} \\
\frac{1}{\lambda^{2} \sigma} \kappa_{3, \lambda} \mathbf{1}^{\top} \mathbf{X} & \frac{1}{\lambda^{2} \sigma} \kappa_{3, \lambda} \mathbf{1}^{\top} \mathbf{N} & \frac{n}{\lambda^{2} \sigma} \kappa_{2, \lambda} & \frac{n}{\lambda^{2}} \kappa_{1, \lambda}
\end{array}\right]
$$

where

$$
\begin{aligned}
\kappa_{1, \lambda} & =\tau_{\lambda}-\frac{2}{\lambda^{2}}\left\{\psi\left(\frac{1}{\lambda^{2}}\right)-\log \left(\frac{1}{\lambda^{2}}\right)\right\}+\frac{6}{\lambda^{2}} r_{\lambda}-\frac{4}{\lambda} u_{\lambda}+v_{\lambda}, \\
\kappa_{2, \lambda} & =u_{\lambda}-\lambda v_{\lambda}-\frac{1}{\lambda}\left\{\psi\left(\frac{1}{\lambda^{2}}\right)-\log \left(\frac{1}{\lambda^{2}}\right)\right\} \\
\kappa_{3, \lambda} & =r_{\lambda}-\lambda u_{\lambda}-1 \\
r_{\lambda} & =E\left[e^{\lambda \epsilon_{i}}\right] \\
u_{\lambda} & =E\left[\epsilon_{i} e^{\lambda \epsilon_{i}}\right] \\
v_{\lambda} & =E\left[\epsilon_{i}^{2} e^{\lambda \epsilon_{i}}\right] \\
\Psi_{\lambda} & =r_{\lambda}+\lambda u_{\lambda}-1 \text { and } \\
\tau_{\lambda} & =1-\frac{1}{\lambda^{2}}\left\{10-12 \log (|\lambda|)-6 \psi\left(\frac{1}{\lambda^{2}}\right)-\frac{4}{\lambda^{2}} \psi^{\prime}\left(\frac{1}{\lambda^{2}}\right)\right\} .
\end{aligned}
$$

By property 8 of chapter 2, we have that

$$
r_{\lambda}=M_{\epsilon}(\lambda)=1
$$

So, a more simplified form of the penalized Fisher information matrix for $\boldsymbol{\theta}$ and $\kappa_{1, \lambda}$ is given by

$$
\mathbf{I}_{p}^{\boldsymbol{\theta} \boldsymbol{\theta}}=\left[\begin{array}{cccc}
\frac{1}{\sigma^{2}} \mathbf{X}^{\top} \mathbf{X} & \frac{1}{\sigma^{2}} \mathbf{X}^{\top} \mathbf{N} & \frac{1}{\sigma^{2}} u_{\lambda} \mathbf{X}^{\top} \mathbf{1} & -\frac{1}{\lambda \sigma} u_{\lambda} \mathbf{X}^{\top} \mathbf{1} \\
\frac{1}{\sigma^{2}} \mathbf{N}^{\top} \mathbf{X} & \frac{1}{\sigma^{2}} \mathbf{N}^{\top} \mathbf{N}+\alpha \mathbf{M} & \frac{1}{\sigma^{2}} u_{\lambda} \mathbf{N}^{\top} \mathbf{1} & -\frac{1}{\lambda \sigma} u_{\lambda} \mathbf{N}^{\top} \mathbf{1} \\
\frac{1}{\sigma^{2}} u_{\lambda} \mathbf{1}^{\top} \mathbf{X} & \frac{1}{\sigma^{2}} u_{\lambda} \mathbf{1}^{\top} \mathbf{N} & \frac{n}{\sigma^{2}}\left(1+v_{\lambda}\right) & \frac{n}{\lambda^{2} \sigma} \kappa_{2, \lambda} \\
-\frac{1}{\lambda \sigma} u_{\lambda} \mathbf{1}^{\top} \mathbf{X} & -\frac{1}{\lambda \sigma} u_{\lambda} \mathbf{1}^{\top} \mathbf{N} & \frac{n}{\lambda^{2} \sigma} \kappa_{2, \lambda} & \frac{n}{\lambda^{2}} \kappa_{1, \lambda}
\end{array}\right]
$$

with

$$
\kappa_{1, \lambda}=\tau_{\lambda}-\frac{2}{\lambda^{2}}\left\{\psi\left(\frac{1}{\lambda^{2}}\right)-\log \left(\frac{1}{\lambda^{2}}\right)\right\}+\frac{6}{\lambda^{2}}-\frac{4}{\lambda} u_{\lambda}+v_{\lambda} .
$$

Remark. This penalized Fisher information matrix generalizes the expression obtained by Paula and Rojas (1997). Such authors worked with the regression model in the parametric situation under the extreme value distributions of type I, which is a particular case of the semi-parametric generalized log-gamma models. 


\subsection{Effective degrees of freedom}

Similarly to additive models, Hastie and Tibshirani (1990) and semi-parametric log-symmetric models, Vanegas and Paula (2015), we can define an approximation of the degree of freedom by analogy with the linear case. One has

$$
\begin{aligned}
\operatorname{df}(\alpha) & =\operatorname{tr}\left[\tilde{\mathbf{X}}\left(\tilde{\mathbf{X}}^{\top} \tilde{\mathbf{X}}+\sigma^{2} \mathbf{M}_{\alpha}\right)^{-1} \tilde{\mathbf{X}}^{\top}\right] \\
& =\operatorname{tr}\left[\left(\mathrm{I}+\sigma^{2} \mathbf{Q}^{-\frac{1}{2}} \mathbf{M}_{\alpha} \mathbf{Q}^{-\frac{1}{2}}\right)^{-1}\right] \\
& =p+\sum_{l=p+1}^{\operatorname{dim}\left(\mathbf{M}_{\alpha}\right)} \frac{1}{1+\phi_{l}},
\end{aligned}
$$

where $\tilde{\mathbf{X}}=(\mathbf{X}, \mathbf{N}), \mathbf{M}_{\alpha}=\operatorname{diag}\left(\mathbf{0}_{p p}, \alpha \mathbf{M}\right), \phi_{l}$ are the eigenvalues of the matrix $\sigma^{2} \mathbf{Q}^{-\frac{1}{2}} \mathbf{M}_{\alpha} \mathbf{Q}^{-\frac{1}{2}}, \mathbf{0}_{p p}$ is a $(p \times p)$ matrix of zeros and $\mathbf{Q}^{\frac{1}{2}} \mathbf{Q}^{\frac{1}{2}}=\tilde{\mathbf{X}}^{\top} \tilde{\mathbf{X}}$. Additionally

$$
\operatorname{dim}\left(\mathbf{M}_{\alpha}\right)>\operatorname{df}(\alpha)>p+\text { zero }
$$

where zero is the number of eigenvalues zero of $\mathbf{M}$. Therefore, one has $\operatorname{df}(\alpha)+2$ parameters to be estimated from the estimating iterative process.

\subsection{Smoothing parameter}

Up to now the smoothing parameter $\alpha$ has been fixed, but in real situations it should be estimated.

One way is to apply the generalized cross-validation $\operatorname{GCV}(\cdot)$ method, see for instance, Wood (2006). This approach consists in minimizing the expected squared-error in predicting a new observation,

$$
\operatorname{GCV}(\alpha)=\frac{n \sum_{i}^{n}\left(\hat{y}_{i}-y_{i}\right)^{2}}{[n-\{p+2+\operatorname{df}(\alpha)\}]^{2}}
$$

where

$$
\hat{y}_{i}=\mathbf{x}_{i}^{\top} \hat{\boldsymbol{\beta}}+\hat{g}\left(t_{i}\right)+\hat{\sigma}\left[\frac{\psi\left(\hat{\lambda}^{-2}\right)-\log \left(\hat{\lambda}^{-2}\right)}{\hat{\lambda}}\right], \text { for } \lambda \neq 0
$$


and

$$
\hat{y}_{i}=\mathbf{x}_{i}^{\top} \hat{\boldsymbol{\beta}}+\hat{g}\left(t_{i}\right), \text { for } \lambda=0,
$$

where $\hat{g}\left(t_{i}\right)=\sum_{j=1}^{q} N_{j}\left(t_{i}\right) \hat{\gamma}_{j}$. This possibility is quite expensive, in a computational way. Alternatively, we may try to minimize the criteria $\operatorname{AIC}(\alpha)$ or $\operatorname{SIC}(\alpha)$. Each criterion guarantees a compromise between model complexity and goodness-of-fit, which is the main goal according with Hastie and Tibshirani (1990); Rigby and Stasinopoulos (2006); Vanegas and Paula (2016) and Wood (2006).

\subsection{Model selection}

For model selection we may apply the Akaike or Schwarz Information Criterion, Akaike (1974) and Schwarz (1978), respectively, which are defined as

$$
\operatorname{AIC}(\alpha)=-2 L_{p}(\boldsymbol{\theta}, \alpha)+2[2+\operatorname{df}(\alpha)]
$$

and

$$
\operatorname{SIC}(\alpha)=-2 L_{p}(\boldsymbol{\theta}, \alpha)+\log (n)[2+\operatorname{df}(\alpha)] .
$$

We choose the model with the smallest AIC or SIC value.

\subsection{Extension for the additive case}

Let $y_{1}, \cdots, y_{n}$ be a set of random variable values and we will assume the following relation between the response values and the explanatory variable values:

$$
y_{i}=\mathbf{x}_{i}^{\top} \boldsymbol{\beta}+g_{1}\left(t_{i 1}\right) \cdots+g_{k}\left(t_{i k}\right)+\sigma \epsilon_{i},
$$

for $i=1, \cdots, n$, where $\mathbf{x}_{i}=\left(x_{i 1}, \cdots, x_{i p}\right)^{\top}$ and $\mathbf{t}_{i}=\left(t_{i 1}, \cdots, t_{i k}\right)^{\top}$ are $(p \times 1)$ and $(k \times 1)$ vectors of explanatory variable values, respectively, $\boldsymbol{\beta}=\left(\beta_{1}, \cdots, \beta_{p}\right)^{\top}$ is a $(p \times 1)$ vector of fixed parameters, $g_{l}(\cdot)$, with $l=1, \cdots, k$, are unknown smoothing functions, $\sigma>0$ and $\epsilon_{i}$ 's are independent identically distributed random errors that follow $\operatorname{GLG}(0,1, \lambda)$. The matrix $\mathbf{X}=\left[\left(\mathbf{x}_{1}^{\top}, \mathbf{t}_{1 .}^{\top}\right), \cdots,\left(\mathbf{x}_{n}^{\top}, \mathbf{t}_{n}^{\top}\right)\right]^{T}$ is assumed to be full column rank. So, $Y_{i}$ has the distribution

$$
Y_{i} \sim \operatorname{GLG}\left(\mathbf{x}_{i}^{\top} \boldsymbol{\beta}+\sum_{l=1}^{k} g_{l}\left(t_{i l}\right), \sigma, \lambda\right),
$$


with

$$
\mathrm{E}\left[Y_{i}\right]=\mathbf{x}_{i}^{\top} \boldsymbol{\beta}+\sum_{l=1}^{k} g_{l}\left(t_{i l}\right)+\sigma\left[\frac{\psi\left(\lambda^{-2}\right)-\log \left(\lambda^{-2}\right)}{|\lambda|}\right]
$$

and

$$
\operatorname{Var}\left[Y_{i}\right]=\sigma^{2} \frac{\psi^{\prime}\left(\lambda^{-2}\right)}{\lambda^{2}}, \text { for } i=1, \cdots, n
$$

where $\psi(\cdot)$ is the digamma function and $\psi^{\prime}(\cdot)$ is the trigamma function.

For estimating the non-parametric functions in model (3.6) we need a way to represent it or at least approximate it. In the next subsection we give two ways to do it.

\subsubsection{Approximating smooth functions}

We are working with two ways of approximating smooth functions, natural cubic splines and P-splines.

\section{Natural cubic splines}

The first step in the natural cubic spline approach of the $l$-th smooth component $g_{l}(\cdot)$ is to identify and ordering the distinct values of $t_{i l},(i=1, \cdots, n)$. Second step is to select the number of knots $q_{l}$ that we will employ. The third step is to select the set of knots $\left\{k_{1 l}, \cdots, k_{q_{l} l}\right\}$ in each continuous component, such that, $k_{1 l}=\min \left(\mathbf{t}_{. l}\right), k_{q_{l} l}=\max \left(\mathbf{t}_{. l}\right)$. So, we have

$$
g_{l}\left(t_{i l}\right)=\sum_{j=1}^{q_{l}} N_{i j l} \gamma_{j l}
$$

approximately, where $N_{i j l}$ is the $(i, j)$-th component of the basis matrix which depends on the spline cubic basis that we had chosen to work with and $\gamma_{j l}$ is the coefficient of $g_{l}(\cdot)$ with respect to the element of the basis at the knot $k_{j l}$. Finally, we can express the natural cubic spline approximation of $g_{l}(t)$ in the point $t$ by

$$
g_{l}(t)=\sum_{j=1}^{q_{l}} N_{j l}(t) \gamma_{j l}
$$


where $N_{j l}(t)$ denotes the $j$-th basis component used to express the $l$-th nonparametric covariate evaluated at the value $t$.

\section{P-splines}

The first step in the P-spline approach is to identify and order the distinct values of $t_{i l},(i=1, \cdots, n)$. Second step is to select the number of internal knots $q_{l}$ for each smooth component $g_{l}(\cdot)$ with $l=1, \cdots, k$. Unlike the cubic spline approach we need to add $2 * o_{l}$ knots, such that, $k_{1+o_{l}}=\min \left(\mathbf{t}_{l l}\right)$ and $k_{q_{l}+o_{l}}=\max \left(\mathbf{t}_{\cdot l}\right)$, where $o_{l}$ is the order of the elements of the B-spline basis. So, we have

$$
g_{l}(t)=\sum_{j=1}^{q_{l}} B_{j l}\left(t, o_{l}-1\right) \gamma_{j l}
$$

approximately, where $B_{j l}\left(\cdot, o_{l}-1\right)$ is the $j$-th element of order $o_{l}$ of the B-spline basis that we had chosen and $\gamma_{j l}$ is the coefficient of $g_{l}(t)$ with respect to this basis.

For both approaches, in matrix notation we have

$$
\mathbf{y}=\mathbf{X} \boldsymbol{\beta}+\mathbf{N}_{1} \gamma_{1}+\cdots+\mathbf{N}_{k} \gamma_{k}+\boldsymbol{\epsilon}
$$

where $\mathbf{y}=\left(y_{1}, \cdots, y_{n}\right)^{\top}, \mathbf{X}$ is an $(n \times p)$ matrix with rows given by $\mathbf{x}_{i}^{\top}, \mathbf{N}_{l}$ are $\left(n \times q_{l}\right)$ basis matrices, $\gamma_{l}=\left(\gamma_{1 l}, \cdots, \gamma_{q_{l} l}\right)^{\top}$ are $\left(q_{l} \times 1\right)$ vectors of parameters and $\boldsymbol{\epsilon}=\left(\epsilon_{1}, \cdots, \epsilon_{n}\right)^{\top}$ is a vector of errors, for $i=1, \cdots, n$ and $l=1, \cdots, k$.

It is important to observe that the parameters in the model above may not be identifiable. So, as pointed up by Wood (2006, section 4.2) and Vanegas and Paula (2016), this problem may solved by applying a reparametrization for the parameters $\boldsymbol{\beta}, \boldsymbol{\gamma}_{1}, \ldots, \boldsymbol{\gamma}_{k}$ by using a QR decomposition approach.

\subsubsection{Penalized log-likelihood function}

The joint density function of a random sample $y_{1}, \cdots, y_{n}$ may be expressed as $f\left(y_{1}, \cdots, y_{n} ; \boldsymbol{\theta}\right)$. The associated $\log$-likelihood function takes the form

$$
L(\boldsymbol{\theta})=n \log \left(\frac{C(\lambda)}{\sigma}\right)+\frac{1}{\lambda} \sum_{i=1}^{n} \epsilon_{i}-\frac{1}{\lambda^{2}} \sum_{i=1}^{n} e^{\lambda \epsilon_{i}}
$$


where $\epsilon_{i}=\frac{y_{i}-\mathbf{x}_{i}^{\top} \boldsymbol{\beta}-\mathbf{N}_{i 1} \gamma_{1}-\cdots \mathbf{N}_{i k} \boldsymbol{\gamma}_{k}}{\sigma}$, and $\mathbf{x}_{i}^{\top}$ and $\mathbf{N}_{i l}$ are rows of the matrices $\mathbf{X}$ and $\mathbf{N}_{l}$, respectively. The penalized log-likelihood function is given by

$L_{p}(\boldsymbol{\theta}, \boldsymbol{\alpha})=n \log \left(\frac{C(\lambda)}{\sigma}\right)+\frac{1}{\lambda} \sum_{i=1}^{n} \epsilon_{i}-\frac{1}{\lambda^{2}} \sum_{i=1}^{n} e^{\lambda \epsilon_{i}}-\frac{\alpha_{1}}{2} \gamma_{1}^{\top} \mathbf{M}_{1} \gamma_{1}-\cdots-\frac{\alpha_{k}}{2} \gamma_{k}^{\top} \mathbf{M}_{k} \gamma_{k}$,

where $\mathbf{M}_{l}$, for $l=1, \cdots, k$, are semi-positive defined matrices which depend on the natural cubic spline basis or B-spline basis we have chosen to work with.

\subsection{Parameter estimation}

In order to perform an iterative procedure for the estimation of $\boldsymbol{\beta}, \gamma_{1}, \cdots, \gamma_{k}, \sigma$ and $\lambda$, we need to derive the penalized score function and the penalized Fisher information matrix, which are derived in Appendix E.

\subsubsection{Penalized score function and penalized Fisher in- formation matrix}

The penalized score function for $\boldsymbol{\theta}$ may be expressed as

$$
\mathbf{U}_{p}^{\theta}=\left[\begin{array}{c}
\mathbf{U}_{p}^{\beta} \\
\mathbf{U}_{p}^{\gamma_{1}} \\
\vdots \\
\mathbf{U}_{p}^{\gamma_{k}} \\
U_{p}^{\sigma} \\
U_{p}^{\lambda}
\end{array}\right]=\left[\begin{array}{c}
-\frac{1}{\lambda \sigma} \mathbf{X}^{\top}\{\mathbf{I}-\mathbf{D}(\mathbf{d})\} \mathbf{1} \\
-\frac{1}{\lambda \sigma} \mathbf{N}_{1}^{\top}\{\mathbf{I}-\mathbf{D}(\mathbf{d})\} \mathbf{1}-\alpha_{1} \mathbf{M}_{1} \boldsymbol{\gamma}_{1} \\
\vdots \\
-\frac{1}{\lambda \sigma} \mathbf{N}_{k}^{\top}\{\mathbf{I}-\mathbf{D}(\mathbf{d})\} \mathbf{1}-\alpha_{k} \mathbf{M}_{k} \gamma_{k} \\
-\frac{1}{\sigma} \mathbf{1}^{\top} \mathbf{1}-\frac{1}{\lambda \sigma} \mathbf{1}^{\top}\{\mathbf{I}-\mathbf{D}(\mathbf{d})\} \boldsymbol{\epsilon} \\
n \zeta_{\lambda}-\frac{1}{\lambda^{2}} \mathbf{1}^{\top} \boldsymbol{\epsilon}+\frac{2}{\lambda^{3}} \mathbf{1}^{\top} \mathbf{D}(\mathbf{d}) \mathbf{1}-\frac{1}{\lambda^{2}} \mathbf{1}^{\top} \mathbf{D}(\mathbf{d}) \boldsymbol{\epsilon}
\end{array}\right]
$$

where $\zeta_{\lambda}=\frac{1}{\lambda}+\frac{2}{\lambda^{3}}\left\{\psi\left(\frac{1}{\lambda^{2}}\right)+2 \log (|\lambda|)-1\right\}, \mathbf{D}(\mathbf{d})$ is a $(n \times n)$ diagonal matrix, $\mathbf{d}=\left(d_{1}, \cdots, d_{n}\right)$ with $d_{i}=e^{\lambda \epsilon_{i}}, \epsilon_{i}=\frac{y_{i}-\mathbf{x}_{i}^{\top} \boldsymbol{\beta}-\mathbf{N}_{i 1} \boldsymbol{\gamma}_{1}-\cdots \mathbf{N}_{i k} \gamma_{k}}{\sigma}$ and $\mathbf{1}$ is an $(n \times 1)$ vector of ones and $\mathbf{I}$ denotes the identity matrix of order $n$.

For convenience of writing, we decompose the penalized Fisher information 
matrix for $\boldsymbol{\theta}$ in two parts, that is, $\mathbf{I}_{p}^{\boldsymbol{\theta} \boldsymbol{\theta}}=\left[\mathbf{I}_{1} \mid \mathbf{I}_{2}\right]$

$$
\mathbf{I}_{1}=\left[\begin{array}{cccc}
\frac{1}{\sigma^{2}} \mathbf{X}^{\top} \mathbf{X} & \frac{1}{\sigma^{2}} \mathbf{X}^{\top} \mathbf{N}_{1} & \cdots & \frac{1}{\sigma^{2}} \mathbf{X}^{\top} \mathbf{N}_{k} \\
\frac{1}{\sigma^{2}} \mathbf{N}_{1}^{\top} \mathbf{X} & \frac{1}{\sigma^{2}} \mathbf{N}_{1}^{\top} \mathbf{N}_{1}+\alpha_{1} \mathbf{M}_{1} & \cdots & \frac{1}{\sigma^{2}} \mathbf{N}_{1}^{\top} \mathbf{N}_{k} \\
\vdots & \vdots & \cdots & \vdots \\
\frac{1}{\sigma^{2}} \mathbf{N}_{k}^{\top} \mathbf{X} & \frac{1}{\sigma^{2}} \mathbf{N}_{k}^{\top} \mathbf{N}_{1} & \cdots & \frac{1}{\sigma^{2}} \mathbf{N}_{k}^{\top} \mathbf{N}_{k}+\alpha_{k} \mathbf{M}_{k} \\
\frac{1}{\sigma^{2}} u_{\lambda} \mathbf{1}^{\top} \mathbf{X} & \frac{1}{\sigma^{2}} u_{\lambda} \mathbf{1}^{\top} \mathbf{N}_{1} & \cdots & \frac{1}{\sigma^{2}} u_{\lambda} \mathbf{1}^{\top} \mathbf{N}_{k} \\
-\frac{1}{\lambda \sigma} u_{\lambda} \mathbf{1}^{\top} \mathbf{X} & -\frac{1}{\lambda \sigma} u_{\lambda} \mathbf{1}^{\top} \mathbf{N}_{1} & \cdots & -\frac{1}{\lambda \sigma} u_{\lambda} \mathbf{1}^{\top} \mathbf{N}_{k}
\end{array}\right]
$$

and

$$
\mathbf{I}_{2}=\left[\begin{array}{cc}
\frac{1}{\sigma^{2}} u_{\lambda} \mathbf{X}^{\top} \mathbf{1} & -\frac{1}{\lambda \sigma} u_{\lambda} \mathbf{X}^{\top} \mathbf{1} \\
\frac{1}{\sigma^{2}} u_{\lambda} \mathbf{N}_{1}^{\top} \mathbf{1} & -\frac{1}{\lambda \sigma} u_{\lambda} \mathbf{N}_{1}^{\top} \mathbf{1} \\
\vdots & \vdots \\
\frac{1}{\sigma^{2}} u_{\lambda} \mathbf{N}_{k}^{\top} \mathbf{1} & -\frac{1}{\lambda \sigma} u_{\lambda} \mathbf{N}_{k}^{\top} \mathbf{1} \\
\frac{n}{\sigma^{2}}\left(1+v_{\lambda}\right) & \frac{n}{\lambda^{2} \sigma} \kappa_{2, \lambda} \\
\frac{n}{\lambda^{2} \sigma} \kappa_{2, \lambda} & \frac{n}{\lambda^{2}} \kappa_{1, \lambda}
\end{array}\right]
$$

with

$$
\kappa_{1, \lambda}=\tau_{\lambda}-\frac{2}{\lambda^{2}}\left\{\psi\left(\frac{1}{\lambda^{2}}\right)-\log \left(\frac{1}{\lambda^{2}}\right)\right\}+\frac{6}{\lambda^{2}}-\frac{4}{\lambda} u_{\lambda}+v_{\lambda}
$$

\subsubsection{Back-fitting algorithm}

In the first step of the iterative process we will remain fixed the parameters $(\sigma, \lambda)$ and will perform a back-fitting process for obtaining the concentrated penalized maximum likelihood estimates for $\left(\boldsymbol{\beta}^{\top}, \boldsymbol{\gamma}_{1}^{\top}, \cdots, \boldsymbol{\gamma}_{k}^{\top}\right)^{\top}$. In the second step, given the obtained estimates, we will perform another iterative process for obtaining the concentrated penalized maximum likelihood estimates for $(\sigma, \lambda)$. Finally, both processes should be alternated until obtaining the joint convergence. We will describe the process for $\lambda \neq 0$. When $\lambda=0$ one has the normal case (see, for instance, Vanegas and Paula (2016)).

Then, in the first step one has the following Fisher scoring algorithm, and using 
the expressions of the subsection 3.3.1, we have that

$$
\begin{aligned}
& {\left[\begin{array}{cccc}
\frac{1}{\sigma^{2}} \mathbf{X}^{\top} \mathbf{X} & \frac{1}{\sigma^{2}} \mathbf{X}^{\top} \mathbf{N}_{1} & \cdots & \frac{1}{\sigma^{2}} \mathbf{X}^{\top} \mathbf{N}_{k} \\
\frac{1}{\sigma^{2}} \mathbf{N}_{1}^{\top} \mathbf{X} & \frac{1}{\sigma^{2}} \mathbf{N}_{1}^{\top^{2}} \mathbf{N}_{1}+\alpha_{1} \mathbf{M}_{1} & \cdots & \frac{1}{\sigma^{2}} \mathbf{N}_{1}^{\top} \mathbf{N}_{k} \\
\vdots & \vdots & \vdots & \vdots \\
\frac{1}{\sigma^{2}} \mathbf{N}_{k}^{\top} \mathbf{X} & \frac{1}{\sigma^{2}} \mathbf{N}_{k}^{\top} \mathbf{N}_{1} & \cdots & \frac{1}{\sigma^{2}} \mathbf{N}_{k}^{\top} \mathbf{N}_{k}+\alpha_{k} \mathbf{M}_{k}
\end{array}\right]^{(l)}\left[\begin{array}{c}
\boldsymbol{\beta}^{l+1}-\boldsymbol{\beta}^{l} \\
\boldsymbol{\gamma}_{1}^{l+1}-\boldsymbol{\gamma}_{1}^{l} \\
\vdots \\
\boldsymbol{\gamma}_{k}^{l+1}-\boldsymbol{\gamma}_{k}^{l}
\end{array}\right]=} \\
& {\left[\begin{array}{c}
-\frac{1}{\lambda \sigma} \mathbf{X}^{\top}\{\mathbf{I}-\mathbf{D}(\mathbf{d})\} \mathbf{1} \\
-\frac{1}{\lambda \sigma} \mathbf{N}_{1}^{\top}\{\mathbf{I}-\mathbf{D}(\mathbf{d})\} \mathbf{1}-\alpha_{1} \mathbf{M}_{1} \boldsymbol{\gamma}_{1} \\
\vdots \\
-\frac{1}{\lambda \sigma} \mathbf{N}_{k}^{\top}\{\mathbf{I}-\mathbf{D}(\mathbf{d})\} \mathbf{1}-\alpha_{k} \mathbf{M}_{k} \boldsymbol{\gamma}_{k}
\end{array}\right]^{(l)} .}
\end{aligned}
$$

Now, we apply the back-fitting method

$$
\left[\begin{array}{c}
\boldsymbol{\beta}^{l+1} \\
\boldsymbol{\gamma}_{\mathbf{1}}^{l+1} \\
\vdots \\
\gamma_{k}^{l+1}
\end{array}\right]=\left[\begin{array}{c}
\left(\mathbf{X}^{\top} \mathbf{X}\right)^{-1} \mathbf{X}^{\top}\left(\mathbf{y}^{l}-\sum_{j \neq 0} \mathbf{N}_{j} \gamma_{j}^{l}\right) \\
\left(\frac{1}{\sigma^{2}} \mathbf{N}_{1}^{\top} \mathbf{N}_{1}+\alpha_{1} \mathbf{M}_{1}\right)^{-1} \frac{1}{\sigma^{2}} \mathbf{N}_{1}^{\top}\left(\mathbf{y}^{l}-\sum_{j \neq 1} \mathbf{N}_{j} \gamma_{j}^{l}\right) \\
\vdots \\
\left(\frac{1}{\sigma^{2}} \mathbf{N}_{k}^{\top} \mathbf{N}_{k}+\alpha_{k} \mathbf{M}_{k}\right)^{-1} \frac{1}{\sigma^{2}} \mathbf{N}_{k}^{\top}\left(\mathbf{y}^{l}-\sum_{j \neq k} \mathbf{N}_{j} \gamma_{j}^{l}\right)
\end{array}\right]
$$

where $\mathbf{y}^{l}=\boldsymbol{\mu}^{l}-\frac{\sigma}{\lambda}\left\{\mathbf{I}-\mathbf{D}\left(\mathbf{d}^{l}\right)\right\} \mathbf{1}$ and $\boldsymbol{\mu}^{l}=\mathbf{X} \boldsymbol{\beta}^{l}+\mathbf{N}_{1}{\gamma_{1}}^{l}+\cdots+\mathbf{N}_{k} \boldsymbol{\gamma}_{k}^{l}$, $\mathbf{d}$ was defined in the subsection 3.8.1. Note that in (3.11) we adopt the notation $\mathbf{N}_{0}=\mathbf{X}$.

The iterative process (3.11) should be alternated with the process

$$
\boldsymbol{\eta}^{(l)}=\operatorname{argmax}_{\eta} L_{p}\left(\boldsymbol{\beta}, \boldsymbol{\gamma}_{1}, \cdots, \boldsymbol{\gamma}_{k}, \boldsymbol{\eta}, \alpha\right),
$$

for $l=1,2, \cdots$, where $\boldsymbol{\eta}=(\sigma, \lambda)^{\top}$.

Then, we propose, for $\alpha_{j}, j=1,2, \cdots, k$ fixed, the following joint iterative process:

1. Give starting values for $\boldsymbol{\beta}, \boldsymbol{\gamma}_{1}, \cdots, \boldsymbol{\gamma}_{k}, \sigma$ and $\lambda$.

2. Given the values for $\sigma$ and $\lambda$ applying the iterative process (3.11) for obtaining the concentrated penalized maximum likelihood estimates of $\boldsymbol{\beta}, \gamma_{1}, \cdots, \gamma_{k}$. 
3. Given the values of $\boldsymbol{\beta}, \boldsymbol{\gamma}_{\mathbf{1}}, \cdots, \boldsymbol{\gamma}_{\boldsymbol{k}}$, obtained at the $k$-th convergence of the iterative process (3.11), obtain the concentrated maximum penalized likelihood estimates of $\sigma$ and $\lambda$ applying (3.12).

4. Iterating between (3.11) and (3.12) until the joint convergence.

Remark. The theoretical foundation of the above algorithm is based on iterative methods for solving non-linear equations, see for instance, Ortega and Rheinboldt (2000). The closed-form expressions for $r_{\lambda}, u_{\lambda}$ and $v_{\lambda}$ which were calculated in the second chapter help us to attain high accuracy for the above algorithm and simplify the expression of the Fisher information matrix.

\subsection{Effective degrees of freedom}

Similarly to additive models, Hastie and Tibshirani (1990) and semi-parametric log-symmetric models, Vanegas and Paula (2016), we can define an approximation of the degree of freedom by analogy with the linear case. We obtain

$$
\begin{aligned}
\operatorname{df}(\boldsymbol{\alpha}) & =\operatorname{tr}\left[\tilde{\mathbf{X}}\left(\tilde{\mathbf{X}}^{\top} \tilde{\mathbf{X}}+\sigma^{2} \mathbf{M}_{\alpha}\right)^{-1} \tilde{\mathbf{X}}^{\top}\right] \\
& =\operatorname{tr}\left[\left(\mathrm{I}+\sigma^{2} \mathbf{Q}^{-\frac{1}{2}} \mathbf{M}_{\alpha} \mathbf{Q}^{-\frac{1}{2}}\right)^{-1}\right] \\
& =p+\sum_{l=p+1}^{\operatorname{dim}\left(\mathbf{M}_{\alpha}\right)} \frac{1}{1+\phi_{l}},
\end{aligned}
$$

where $\tilde{\mathbf{X}}=\left(\mathbf{X}^{\top}, \mathbf{N}_{1}^{\top}, \cdots, \mathbf{N}_{k}^{\top}\right)^{\top}, \mathbf{M}_{\alpha}=\operatorname{diag}\left(\mathbf{0}_{p p}, \alpha_{1} \mathbf{M}_{1}, \cdots, \alpha_{k} \mathbf{M}_{k}\right), \phi_{l}$ are the eigenvalues of the matrix $\sigma^{2} \mathbf{Q}^{-\frac{1}{2}} \mathbf{M}_{\alpha} \mathbf{Q}^{-\frac{1}{2}}, \mathbf{0}_{p p}$ is a $(p \times p)$ matrix of zeros and $\mathbf{Q}^{\frac{1}{2}} \mathbf{Q}^{\frac{1}{2}}=\tilde{\mathbf{X}}^{\top} \tilde{\mathbf{X}}$. Additionally

$$
\operatorname{dim}\left(\mathbf{M}_{\alpha}\right)>\operatorname{df}(\alpha)>p+\sum_{j=1}^{k} \text { zero }_{j}
$$

where zero $j$ is the number of eigenvalues zero of $\mathbf{M}_{j}, j=1, \cdots, k$. Therefore, one has $\operatorname{df}(\alpha)+2$ parameters to be estimated from the estimating iterative process. 


\subsection{Smoothing parameter}

Up to now the smoothing parameters $\alpha_{j}, j=1, \cdots, k$, have been fixed, but in real situations they should be estimated.

One way is to apply a multivariate version of the generalized cross-validation GCV (.) method, see for instance,Wood (2006). This approach consists in minimizing the expected squared-error in predicting a new observation, that is

$$
\operatorname{GCV}\left(\alpha_{1}, \cdots, \alpha_{k}\right)=\frac{n \sum_{i}^{n}\left(\hat{y}_{i}-y_{i}\right)^{2}}{\left[n-\left\{2+\sum_{j=1}^{k} \operatorname{df}\left(\alpha_{j}\right)\right\}\right]^{2}},
$$

where

$$
\hat{y}_{i}=\mathbf{x}_{i}^{\top} \hat{\boldsymbol{\beta}}+\sum_{j=1}^{k} \hat{g}_{i j}+\hat{\sigma}\left[\frac{\psi\left(\hat{\lambda}^{-2}\right)-\log \left(\hat{\lambda}^{-2}\right)}{\hat{\lambda}}\right], \text { for } \lambda \neq 0
$$

and

$$
\hat{y}_{i}=\mathbf{x}_{i}^{\top} \hat{\boldsymbol{\beta}}+\sum_{j=1}^{k} \hat{g}_{i j}, \text { for } \lambda=0
$$

where $\hat{g}_{j}\left(t_{i j}\right)=\mathbf{N}_{i j} \hat{\gamma}_{j}$, for $i=1, \cdots, n$ and $j=1, \cdots, k$. This possibility is quite expensive in a computational way. Alternatively, we can try to minimize the criteria $\operatorname{AIC}(\boldsymbol{\alpha})$ or $\operatorname{SIC}(\boldsymbol{\alpha})$. Each criterion guarantees a compromise between model complexity and goodness-of-fit, which is the main goal according with Hastie and Tibshirani (1990); Rigby and Stasinopoulos (2006); Vanegas and Paula (2016) and Wood (2006).

\subsection{Model selection}

For model selection we may apply the same expression of the section 3.5 and choose the model with the smallest $\operatorname{AIC}(\boldsymbol{\alpha})$ or $\operatorname{SIC}(\boldsymbol{\alpha})$ value, where $\boldsymbol{\alpha}=$ $\left(\alpha_{1}, \cdots, \alpha_{k}\right)$

\subsection{Asymptotic statements}

Lehmann and Casella (1998) pointed out the following important issue: "to evaluate the limit behavior of the estimators is necessary to provide an essential 
complement to the small-sample behavior. Sometimes we can see properties of the estimators that are behind of the small-sample context."To study the limit behavior of the penalized maximum likelihood estimators $\hat{\boldsymbol{\theta}}$, we adopt the approach of Vanegas and Paula (2016). This approach consists in taking the number of knots fixed, which means that the penalty matrices and the size of $\gamma_{j}$, for $j=1, \cdots, k$, are not functions of the sample size $n$. Some additional conditions are necessary to guarantee the consistence and asymptotic normality of the estimators.

1. There is $\boldsymbol{\gamma}_{j}^{0} \in \mathbf{R}^{q_{j}-1}$ such that $g_{j}(t)=\mathbf{N}_{j}(t) \boldsymbol{\gamma}_{j}^{0}, \forall t \in I_{j}$ and $j=$ $1, \cdots, k$. Hence, $\gamma^{0}=\left(\left(\gamma_{1}^{0}\right)^{\top}, \cdots,\left(\gamma_{k}^{0}\right)^{\top}\right)^{\top}$ is the true parameter vector for $\left(g_{1}, \cdots, g_{k}\right)^{\top}$;

2. $n^{-1} \mathbf{K}_{n}\left(\boldsymbol{\theta}_{0}\right) \rightarrow \Sigma\left(\boldsymbol{\theta}_{0}\right)$, where $\boldsymbol{\theta}_{0}=\left(\left(\boldsymbol{\beta}^{0}\right)^{\top},\left(\boldsymbol{\gamma}^{0}\right)^{\top}, \sigma^{0}, \lambda^{0}\right)^{\top}$ is the true parameter vector for $\boldsymbol{\theta}_{0}$;

3. $\mathbf{K}_{n}^{-\frac{1}{2}}\left(\boldsymbol{\theta}_{0}\right) \mathbf{U}_{n}\left(\boldsymbol{\theta}_{0}\right) \underset{\longrightarrow}{\longrightarrow} \boldsymbol{N}(\mathbf{0}, \mathbf{I})$.

4. $n^{-1} \mathbf{U}_{n}\left(\boldsymbol{\theta}_{0}\right) \stackrel{P}{\longrightarrow} \mathbf{0}$

5. $n^{-1}\left\{\left.\frac{\partial L(\theta)}{\partial \theta \partial \theta^{\top}}\right|_{\boldsymbol{\theta}=\boldsymbol{\theta}_{0}}+\mathbf{K}_{n}\left(\boldsymbol{\theta}_{0}\right)\right\} \stackrel{P}{\longrightarrow} \mathbf{0}$

6. $n^{-1}\left\{\left.\frac{\partial L(\theta)}{\partial \theta \partial \theta^{\top}}\right|_{\boldsymbol{\theta}=\boldsymbol{\theta}^{*}}-\left.\frac{\partial L(\theta)}{\partial \theta \partial \theta^{\top}}\right|_{\boldsymbol{\theta}=\boldsymbol{\theta}_{0}}\right\} \stackrel{P}{\longrightarrow} \mathbf{0}$, for all $\boldsymbol{\theta}^{*}$ in line segment joining $\boldsymbol{\theta}_{0}$ and $\boldsymbol{\theta}^{*}$;

7. The smoothing parameter may dependent on the sample size. For a sample of size $n$, let $\boldsymbol{\alpha}_{n}$ be the smoothing parameter. So, $\left\|\boldsymbol{\alpha}_{n}\right\| \rightarrow r$ with $r<\infty$.

In addition

$$
\begin{gathered}
\mathbf{U}_{p}^{\boldsymbol{\theta}}\left(\boldsymbol{\theta}_{0}\right)=\mathbf{U}_{n}\left(\boldsymbol{\theta}_{0}\right)-\tilde{\mathbf{M}}_{\boldsymbol{\alpha}} \\
\mathbf{I}_{p}^{\boldsymbol{\theta} \boldsymbol{\theta}}\left(\boldsymbol{\theta}_{0}\right)=\mathbf{K}_{n}\left(\boldsymbol{\theta}_{0}\right)+\tilde{\mathbf{M}}_{\boldsymbol{\alpha}}
\end{gathered}
$$

where $\mathbf{K}_{n}\left(\boldsymbol{\theta}_{0}\right)=\left.E\left\{-\frac{\partial^{2} L(\boldsymbol{\theta})}{\partial \boldsymbol{\theta} \partial \boldsymbol{\theta}}\right\}\right|_{\boldsymbol{\theta}=\boldsymbol{\theta}_{0}}$ is a positive definite matrix and $\tilde{\mathbf{M}}_{\boldsymbol{\alpha}}=$ $\operatorname{diag}\left(\mathbf{M}_{\boldsymbol{\alpha}}, \mathbf{0}_{2}\right)$.

Theorem. Under the conditions (1) - (7) we may prove that $\hat{\boldsymbol{\theta}}$ is a consistent estimator, and

$$
\mathbf{K}_{n}^{-\frac{1}{2}}\left(\boldsymbol{\theta}_{0}\right)\left\{\mathbf{K}_{n}\left(\boldsymbol{\theta}_{0}\right)+\tilde{\mathbf{M}}_{\boldsymbol{\alpha}}\right\}\left(\hat{\boldsymbol{\theta}}-\boldsymbol{\theta}_{0}\right) \stackrel{D}{\longrightarrow} \boldsymbol{N}(\mathbf{0}, \mathbf{I}) .
$$


The proof is quite analogous to the one given in appendix of Vanegas and Paula (2016). Therefore, we may derive the asymptotic variance-covariance matrix of $\hat{\boldsymbol{\theta}}$ as

$$
\operatorname{Var}(\hat{\boldsymbol{\theta}})=\left\{\mathbf{I}_{p}^{\boldsymbol{\theta} \boldsymbol{\theta}}\right\}^{-1} \mathbf{K}_{n}(\boldsymbol{\theta})\left\{\mathbf{I}_{p}^{\boldsymbol{\theta} \boldsymbol{\theta}}\right\}^{-1}
$$

\subsection{Confidence band for a non-parametric com- ponent}

There is possible to build a confidence band for each non-parametric component $g_{1}, \cdots, g_{k}$ of the model (3.6). We adopt the approach of Vanegas and Paula (2016) for this construction. Let denote $\alpha$ and $\alpha^{\prime}$ confidence levels. Based on the asymptotic properties, we have confidence intervals $\mathbf{C I}_{1, j}^{\alpha}, \cdots, \mathbf{C I}_{n, j}^{\alpha}$ for $g_{j}\left(t_{i j}\right), i=1, \cdots, n, j=1, \cdots, k$, as

$$
\mathbf{C I}_{i, j}^{\alpha}=\left\{x \in \mathbf{R}:\left|\hat{g}_{j}\left(t_{i j}\right)-x\right| \leq \Phi^{-1}\left(1-\frac{\alpha}{2}\right) \widehat{\operatorname{Var}}\left\{\hat{g}_{j}\left(t_{i j}\right)\right\}^{\frac{1}{2}}\right\} .
$$

The simultaneous confidence level of $\mathbf{C I}_{1, j}^{\alpha}, \cdots, \mathbf{C I}_{n, j}^{\alpha}$ is given by $P\left[\bigcap_{l=1}^{n} \mathbf{C I}_{l, j}^{\alpha}\right]$ and we have that

$$
P\left[\bigcap_{i=1}^{n} \mathbf{C I}_{l, j}^{\alpha}\right] \geq 1-q_{j} \alpha
$$

where $q_{j}$ was defined in subsection 3.7.1. From Bonferroni's method (see, for instance, Shao (2003)), we take $\alpha=\frac{\alpha^{\prime}}{q_{j}}$. So, the simultaneous confidence level $\mathbf{C I}_{1, j}^{\alpha}, \cdots, \mathbf{C I}_{n, j}^{\alpha}$ is at least $100\left(1-\alpha^{\prime}\right) \%$. 


\section{Chapter 4}

\section{Inference procedures: censored case}

In this chapter we will work with semi-parametric generalized log-gamma regression models under the setting of censored data. This is a natural extension of survival parametric regression models for the analysis of censored data, which in the last years are shown to be useful (see, for instance, Cox et al. (2007), Agostinelli et al. (2014)). We will consider censoring under the assumption of independent random censoring. The expression for the observed information matrix is consistent with the one given in Ortega et al. (2008).

\subsection{Introduction}

Let $\tilde{T}_{1}, \cdots, \tilde{T}_{n}$ be failure times or lifetime of $n$ observational units. These failure times are described by independent, strictly positive and asymmetric random variables with distribution generalized gamma, that is, $\tilde{T} \sim \mathrm{GG}(\mu, \sigma, \lambda)$, see property 9 , chapter 2 . We assume that $\tilde{T}_{i}$ may be expressed as

$$
\tilde{T}_{i}=e^{\mu_{i}} \eta_{i}^{\sigma}, \quad i=1, \cdots, n
$$

where $\eta_{1}, \cdots, \eta_{n}$ are independent and multiplicative random errors distributed, $\mathrm{GG}(0,1, \lambda)$, a generalized gamma distribution with probability density function given by

$$
f_{\tilde{T}}(t ; \lambda)=\frac{|\lambda|}{t \Gamma\left(\lambda^{-2}\right)}\left(\lambda^{-2}\right)^{\lambda^{-2}} t^{\frac{1}{\lambda}} e^{-\lambda^{-2} t^{\lambda}}
$$


For the survival and hazard functions of $\tilde{T}$ see property 10, chapter 2.

We may formulate the following model:

$$
y_{i}=\log \left(\tilde{T}_{i}\right)=\mu_{i}+\sigma \epsilon_{i}, \quad i=1, \cdots, n,
$$

where $\mu_{i}$ and $\sigma$ are, respectively, the location and scale parameters of $y_{i}$ and $\epsilon_{1}=\log \left(\eta_{1}\right), \cdots, \epsilon_{n}=\log \left(\eta_{n}\right)$ are independent and identically additive errors distributed as $\operatorname{GLG}(0,1, \lambda)$.

Now, we are describing the assumptions behind the censoring times $C_{1}, \cdots, C_{n}$. The first assumption on the censoring times is that they are positive, continuous and independent random variables. The next assumption on the censoring times is that the parameters which identify their probability density functions do not have any information with respect to the parameters of interest. The third assumption is the statistical independence between $C_{1}, \cdots, C_{n}$ and $\tilde{T}_{1}, \cdots, \tilde{T}_{n}$. Therefore, we have $n$ random bivariate vectors $\left(T_{1}, \delta_{1}\right), \cdots,\left(T_{n}, \delta_{n}\right)$ following either of the next schemes:

- $T_{i}=\min \left(\tilde{T}_{i}, C_{i}\right)$ and $\tilde{\delta}=\mathrm{I}\left(\tilde{T}_{i}>C_{i}\right)$ for right censoring scheme and

- $T_{i}=\max \left(\tilde{T}_{i}, C_{i}\right)$ and $\tilde{\delta}=\mathrm{I}\left(\tilde{T}_{i}<C_{i}\right)$ for left censoring scheme,

where $\mathrm{I}(\cdot)$ denotes the indicator function. The observed bivariate data, denoted by $\left(t_{1}, \delta_{1}\right), \cdots,\left(t_{n}, \delta_{n}\right)$ are realizations from one of the previous schemes. The property 2 of the chapter 2 implies the closure under reciprocals of generalized gamma variables. Hence, if we want to model a set of observations $\left(t_{1}, \delta_{1}\right), \cdots,\left(t_{n}, \delta_{n}\right)$ from the left censoring scheme we may equivalently to analyze the set $\left(1 / t_{1}, \delta_{1}\right), \cdots,\left(1 / t_{n}, \delta_{n}\right)$ under the right censoring scheme.

\subsection{Censored partially linear generalized log- gamma regression models}

\subsubsection{The model}

Let $y_{i}$ be either the observed log-lifetime or log-censoring time for the $i$ th observation. We propose the model

$$
y_{i}=\mathbf{x}_{i}^{\top} \boldsymbol{\beta}+g\left(s_{i}\right)+\sigma \epsilon_{i}
$$


for $i=1, \cdots, n$, where $\mathbf{x}_{i}=\left(x_{i 1}, \cdots, x_{i p}\right)^{\top}$ is a $(p \times 1)$ vector of explanatory variable values, $s_{i}$ is an observed value of a continuous covariate, $\boldsymbol{\beta}=\left(\beta_{1}, \cdots, \beta_{p}\right)^{\top}$ is a $(p \times 1)$ vector of fixed parameters, $g(\cdot)$ is an unknown smoothing functions, $\sigma>0$ and $\epsilon_{i}$ 's are independent identically distributed random errors that follow $\operatorname{GLG}(0,1, \lambda)$. The matrix $\mathbf{X}=\left[\left(\mathbf{x}_{1}^{\top}, s_{1}\right), \cdots,\left(\mathbf{x}_{n}^{\top}, s_{n}\right)\right]^{\top}$ is assumed to be full column rank.

Like the uncensored case for estimating the continuous covariate in model (4.1) we need a way to represent it or at least approximate it. We may use the same two forms of the chapter 3. That is, through natural cubic splines or P-splines.

\subsubsection{The likelihood}

Let $y_{i}$ be either the observed log-lifetime or log-censoring time for the $i$-th observation. In this case, the likelihood function takes the following form:

$$
L\left(y_{1}, \cdots, y_{n} ; \boldsymbol{\theta}\right)=\prod_{i=1}^{n} f\left(y_{i} ; \boldsymbol{\theta}\right)^{1-\delta_{i}} S\left(y_{i} ; \boldsymbol{\theta}\right)^{\delta_{i}},
$$

where

$$
\delta_{i}= \begin{cases}0 & \text { if } t_{i} \text { is a failure time } \\ 1 & \text { if } t_{i} \text { is a censored time }\end{cases}
$$

The respective log-likelihood function takes the form

$$
L(\boldsymbol{\theta})=\sum_{i \in D}\left[\log \left(\frac{c(\lambda)}{\sigma}\right)+\frac{1}{\lambda} \epsilon_{i}-\frac{1}{\lambda^{2}} e^{\lambda \epsilon_{i}}\right]+\sum_{i \in C} \log \left\{S\left(\epsilon_{i} ; \lambda\right)\right\} .
$$

The index set $D$ corresponds to uncensored individuals, whereas the index set $C$ corresponds to censored individuals, $\epsilon_{i}$ are i.i.d. random variables that are distributed as $\operatorname{GLG}(0,1, \lambda)$ and $S\left(\epsilon_{i} ; \lambda\right)$ denotes the survival function which was defined in the section 4.2.2 and assumes the following form:

$$
S\left(\epsilon_{i} ; \lambda\right)=\left\{\begin{array}{l}
1-\mathrm{I}\left(\lambda^{-2}, \lambda^{-2} e^{\lambda \epsilon_{i}}\right), \quad \text { if } \lambda>0 \\
\mathrm{I}\left(\lambda^{-2}, \lambda^{-2} e^{\lambda \epsilon_{i}}\right), \quad \text { if } \lambda<0,
\end{array}\right.
$$

where $\mathrm{I}(\lambda, a)$ denotes the incomplete gamma function,

$$
\mathrm{I}(\lambda, a)=\frac{1}{\Gamma(\lambda)} \int_{0}^{a} x^{\lambda-1} e^{-x} d x .
$$

The previous expression for the survival function is a consequence of the property 3 in chapter 2 . 


\subsection{Penalized log-likelihood function}

In this section we will introduce the concept of penalized log-likelihood function for semi-parametric generalized log-gamma regression models under the presence of censored data. Since the survival function of a generalized log-gamma variable depends on the sign of $\lambda$, we consider two situations described below. When $\lambda>0$,

$$
\begin{aligned}
L_{p}(\boldsymbol{\theta}, \alpha) & =\sum_{i \in D}\left[\log \left(\frac{c(\lambda)}{\sigma}\right)+\frac{1}{\lambda} \epsilon_{i}-\frac{1}{\lambda^{2}} e^{\lambda \epsilon_{i}}\right]+\sum_{i \in C} \log \left\{1-\mathrm{I}\left(\lambda^{-2}, \lambda^{-2} e^{\lambda \epsilon_{i}}\right)\right\} \\
& -\frac{\alpha}{2} \sum_{i=1}^{q} \sum_{j=1}^{q} M_{i j} \gamma_{i} \gamma_{j} .
\end{aligned}
$$

When $\lambda<0$,

$$
\begin{aligned}
L_{p}(\boldsymbol{\theta}, \alpha) & =\sum_{i \in D}\left[\log \left(\frac{c(\lambda)}{\sigma}\right)+\frac{1}{\lambda} \epsilon_{i}-\frac{1}{\lambda^{2}} e^{\lambda \epsilon_{i}}\right]+\sum_{i \in C} \log \left\{\mathrm{I}\left(\lambda^{-2}, \lambda^{-2} e^{\lambda \epsilon_{i}}\right)\right\} \\
& -\frac{\alpha}{2} \sum_{i=1}^{q} \sum_{j=1}^{q} M_{i j} \gamma_{i} \gamma_{j}
\end{aligned}
$$

where $\epsilon_{i}=\frac{y_{i}-\mathbf{x}_{i}^{\top} \beta-\mathbf{N}_{i} \gamma}{\sigma}$ and $M_{i j}$ is the $(i, j)$-th element of the non-negative definite penalization matrix $\mathbf{M}$ associated with the non-parametric component $g(\cdot)$.

\subsubsection{Parameter estimation}

The direct application of the Newton-Raphson method in this case have some inconvenient. However, it may fixed, see for instance section 8.2 of Ortega and Rheinboldt (2000), with a damped Newton method.

So, for the maximization of one of the expressions in the subsection 4.3.1 we follow two steps. According with Ortega et al. (2008) it is reasonable to expect that the shape parameter $\lambda$ belongs to the interval $[-3,3]$. Hence, the first step is to obtain $\hat{\lambda}$ from an auxiliary function shape.parameter. Then, we find the maximum likelihood estimates $\beta_{\hat{\lambda}}$ and $\sigma_{\hat{\lambda}}$. In this step, we have built the $\mathrm{R}$ function ssurvglg which gives us this and the related results, (see chapter 6 for details about these and others $\mathbf{R}$ functions developed in this work). 


\subsubsection{Penalized score function}

For $\lambda>0$ the components of the penalized score function of $\boldsymbol{\theta}$ may be expressed as

$$
\begin{aligned}
& U_{p}^{\beta_{j}}=-\frac{1}{\lambda \sigma} \sum_{i \in D} x_{i j}\left[1-e^{\lambda \epsilon_{i}}\right]+\frac{\lambda}{\sigma \Gamma\left(\lambda^{-2}\right)} \sum_{i \in C}\left[x_{i j} \frac{a_{i}^{\lambda^{-2}} e^{-a_{i}}}{1-\mathrm{I}\left(\lambda^{-2}, a_{i}\right)}\right], \\
& U_{p}^{\gamma_{j}}=-\frac{1}{\lambda \sigma} \sum_{i \in D} N_{i j}\left[1-e^{\lambda \epsilon_{i}}\right]+\frac{\lambda}{\sigma \Gamma\left(\lambda^{-2}\right)} \sum_{i \in C}\left[N_{i j} \frac{a_{i}^{\lambda^{-2}} e^{-a_{i}}}{1-\mathrm{I}\left(\lambda^{-2}, a_{i}\right)}\right]-\alpha \sum_{i=1}^{q} M_{i j} \gamma_{i}
\end{aligned}
$$

with $j=1, \cdots, q$ and

$$
\begin{aligned}
U_{p}^{\sigma} & =-\frac{1}{\sigma} \sum_{i \in D}\left[1+\frac{1}{\lambda}\left(\epsilon_{i}-\epsilon_{i} e^{\lambda \epsilon_{i}}\right)\right]+\frac{\lambda}{\sigma \Gamma\left(\lambda^{-2}\right)} \sum_{i \in C}\left[\epsilon_{i} \frac{a_{i}^{\lambda^{-2}} e^{-a_{i}}}{1-\mathrm{I}\left(\lambda^{-2}, a_{i}\right)}\right] \text { and } \\
U_{p}^{\lambda} & =\sum_{i \in D}\left[\zeta_{\lambda}-\frac{1}{\lambda^{2}} \epsilon_{i}+\frac{2}{\lambda^{3}} e^{\lambda \epsilon_{i}}-\frac{1}{\lambda^{2}} \epsilon_{i} e^{\lambda \epsilon_{i}}\right] \\
& +\sum_{i \in C}-\frac{2 \lambda^{-3} \psi\left(\lambda^{-2}\right) I\left(\lambda^{-2}, a_{i}\right)+\frac{1}{\Gamma\left(\lambda^{-2}\right)} a_{i}^{\lambda^{-2}} e^{-a_{i}}\left(\epsilon_{i}-2 \lambda^{-1}\right)}{1-\mathrm{I}\left(\lambda^{-2}, a_{i}\right)} .
\end{aligned}
$$

For $\lambda<0$ the components of the penalized score function of $\boldsymbol{\theta}$ may be expressed as

$$
\begin{aligned}
& U_{p}^{\beta_{j}}=-\frac{1}{\lambda \sigma} \sum_{i \in D} x_{i j}\left[1-e^{\lambda \epsilon_{i}}\right]+\frac{-\lambda}{\sigma \Gamma\left(\lambda^{-2}\right)} \sum_{i \in C}\left[x_{i j} \frac{a_{i}^{\lambda^{-2}} e^{-a_{i}}}{\mathrm{I}\left(\lambda^{-2}, a_{i}\right)}\right] \\
& U_{p}^{\gamma_{j}}=-\frac{1}{\lambda \sigma} \sum_{i \in D} N_{i j}\left[1-e^{\lambda \epsilon_{i}}\right]+\frac{-\lambda}{\sigma \Gamma\left(\lambda^{-2}\right)} \sum_{i \in C}\left[N_{i j} \frac{a_{i}^{\lambda^{-2}} e^{-a_{i}}}{\mathrm{I}\left(\lambda^{-2}, a_{i}\right)}\right]-\alpha \sum_{i=1}^{q} M_{i j} \gamma_{i},
\end{aligned}
$$

with $j=1, \cdots, q$ and 


$$
\begin{aligned}
U_{p}^{\sigma} & =-\frac{1}{\sigma} \sum_{i \in D}\left[1+\frac{1}{\lambda}\left(\epsilon_{i}-\epsilon_{i} e^{\lambda \epsilon_{i}}\right)\right]+\frac{-\lambda}{\sigma \Gamma\left(\lambda^{-2}\right)} \sum_{i \in C} \frac{\epsilon_{i} a_{i}^{\lambda^{-2}} e^{-a_{i}}}{\mathrm{I}\left(\lambda^{-2}, a_{i}\right)} \text { and } \\
U_{p}^{\lambda} & =\sum_{i \in D}\left[\zeta_{\lambda}-\frac{1}{\lambda^{2}} \epsilon_{i}+\frac{2}{\lambda^{3}} e^{\lambda \epsilon_{i}}-\frac{1}{\lambda^{2}} \epsilon_{i} e^{\lambda \epsilon_{i}}\right] \\
& +\sum_{i \in C} \frac{2 \lambda^{-3} \psi\left(\lambda^{-2}\right) I\left(\lambda^{-2}, a_{i}\right)+\frac{1}{\Gamma\left(\lambda^{-2}\right)} a_{i}^{\lambda^{-2}} e^{-a_{i}}\left(\epsilon_{i}-2 \lambda^{-1}\right)}{\mathrm{I}\left(\lambda^{-2}, a_{i}\right)}
\end{aligned}
$$

where $\zeta_{\lambda}=\frac{1}{\lambda}+\frac{2}{\lambda^{3}}\left\{\psi\left(\frac{1}{\lambda^{2}}\right)+2 \log (|\lambda|)-1\right\}, a_{i}=\lambda^{-2} e^{\lambda \epsilon_{i}}$ and $\mathbf{D}(\mathbf{d})$ is a $(n \times n)$ diagonal matrix with elements $\mathbf{d}=\left(d_{1}, \cdots, d_{n}\right), d_{i}=e^{\lambda \epsilon_{i}}$ for $i=1, \cdots, n$.

\subsection{Smoothing parameter}

Up to now the smoothing parameters $\alpha$, has been fixed, but in real situations it should be estimated. In chapter 3 was explained the drawbacks if we want to apply a multivariate version of the generalized cross-validation GCV $(\cdot)$ method. So, we minimize the information measure $\operatorname{AIC}(\alpha)$ or $\operatorname{SIC}(\alpha)$. These criteria guarantee a compromise between model complexity and goodness-of-fit, which is the main goal according with Hastie and Tibshirani (1990); Rigby and Stasinopoulos (2006); Vanegas and Paula (2016) and Wood (2006).

\subsection{Model selection}

For model selection we may apply the Akaike or Schwarz Information Criterion Akaike (1974); Schwarz (1978), respectively, which are defined as

$$
\operatorname{AIC}(\alpha)=-2 L_{p}(\boldsymbol{\theta}, \alpha)+2[2+\operatorname{df}(\alpha)]
$$

and

$$
\operatorname{SIC}(\alpha)=-2 L_{p}(\boldsymbol{\theta}, \alpha)+\log (n)[2+\operatorname{df}(\alpha)]
$$


We choose the model with the smallest AIC or SIC value.

\subsection{Extension for the additive case}

$$
y_{i}=\mathbf{x}_{i}^{\top} \boldsymbol{\beta}+\sum_{j=1}^{k} g_{j}\left(s_{i j}\right)+\sigma \epsilon_{i}
$$

for $i=1, \cdots, n$, where $\mathbf{x}_{i}=\left(x_{i 1}, \cdots, x_{i p}\right)^{\top}$ and $\mathbf{s}_{i}=\left(s_{i 1}, \cdots, s_{i k}\right)^{\top}$ are $(p \times 1)$ and $(k \times 1)$ vectors of explanatory variable values, respectively, $\boldsymbol{\beta}=\left(\beta_{1}, \cdots, \beta_{p}\right)^{\top}$ is a $(p \times 1)$ vector of fixed parameters, $g_{j}(\cdot), j=1 \cdots, k$, are unknown smoothing functions, $\sigma>0$ and $\epsilon_{i}$ 's are independent identically distributed random errors that follow $\operatorname{GLG}(0,1, \lambda)$. The matrix $\mathbf{X}=\left[\left(\mathbf{x}_{1}^{\top}, \mathbf{s}_{1}^{\top}\right), \cdots,\left(\mathbf{x}_{n}^{\top}, \mathbf{s}_{n}^{\top}\right)\right]^{\top}$ is assumed to be full column rank.

\subsection{The likelihood}

Let $y_{i}$ be either the observed log-lifetime or log-censoring time for the $i$-th observation. In this case, the likelihood function takes the following form:

$$
L\left(y_{1}, \cdots, y_{n} ; \boldsymbol{\theta}\right)=\prod_{i=1}^{n} f\left(y_{i} ; \boldsymbol{\theta}\right)^{1-\delta_{i}} S\left(y_{i} ; \boldsymbol{\theta}\right)^{\delta_{i}}
$$

where

$$
\delta_{i}= \begin{cases}0 & \text { if } t_{i} \text { is a failure time } \\ 1 & \text { if } t_{i} \text { is a censored time }\end{cases}
$$

The respective log-likelihood function takes the form

$$
L(\boldsymbol{\theta})=\sum_{i \in D}\left[\log \left(\frac{c(\lambda)}{\sigma}\right)+\frac{1}{\lambda} \epsilon_{i}-\frac{1}{\lambda^{2}} e^{\lambda \epsilon_{i}}\right]+\sum_{i \in C} \log \left\{S\left(\epsilon_{i} ; \lambda\right)\right\} .
$$

The index set $D$ corresponds to uncensored individuals, whereas the index set $C$ corresponds to censored individuals, $\epsilon_{i}$ are i.i.d. random variables that are distributed as $\operatorname{GLG}(0,1, \lambda)$ and $S\left(\epsilon_{i} ; \lambda\right)$ denotes the survival function which was defined in section 4.3 . 


\subsubsection{Penalized log-likelihood function}

In this section we will introduce the concept of penalized log-likelihood function for semi-parametric generalized log-gamma regression models under the presence of censored data. Since the survival function of a generalized log-gamma variable depends on the sign of $\lambda$, we consider two situations described below. When $\lambda>0$,

$$
\begin{aligned}
L_{p}(\boldsymbol{\theta}, \boldsymbol{\alpha}) & =\sum_{i \in D}\left[\log \left(\frac{c(\lambda)}{\sigma}\right)+\frac{1}{\lambda} \epsilon_{i}-\frac{1}{\lambda^{2}} e^{\lambda \epsilon_{i}}\right]+\sum_{i \in C} \log \left(1-\mathrm{I}\left(\lambda^{-2}, \lambda^{-2} e^{\lambda \epsilon_{i}}\right)\right) \\
& -\frac{\alpha_{1}}{2} \sum_{i=1}^{q_{1}} \sum_{j=1}^{q_{1}} \mathrm{M}_{i j 1} \gamma_{i 1} \gamma_{j 1}-\cdots-\frac{\alpha_{k}}{2} \sum_{i=1}^{q_{k}} \sum_{j=1}^{q_{k}} \mathrm{M}_{i j k} \gamma_{i k} \gamma_{j k} .
\end{aligned}
$$

When $\lambda<0$,

$$
\begin{aligned}
L_{p}(\boldsymbol{\theta}, \boldsymbol{\alpha}) & =\sum_{i \in D}\left[\log \left(\frac{c(\lambda)}{\sigma}\right)+\frac{1}{\lambda} \epsilon_{i}-\frac{1}{\lambda^{2}} e^{\lambda \epsilon_{i}}\right]+\sum_{i \in C} \log \left(\mathrm{I}\left(\lambda^{-2}, \lambda^{-2} e^{\lambda \epsilon_{i}}\right)\right) \\
& -\frac{\alpha_{1}}{2} \sum_{i=1}^{q_{1}} \sum_{j=1}^{q_{1}} \mathrm{M}_{i j 1} \gamma_{i 1} \gamma_{j 1}-\cdots-\frac{\alpha_{k}}{2} \sum_{i=1}^{q_{k}} \sum_{j=1}^{q_{k}} \mathrm{M}_{i j k} \gamma_{i k} \gamma_{j k} .
\end{aligned}
$$

where $\epsilon_{i}=\frac{y_{i}-\mathbf{X}_{i}^{\top} \beta-\mathbf{N}_{i 1} \gamma_{1}-\cdots \mathbf{N}_{i k} \gamma_{k}}{\sigma}$ and $M_{i j l}$ is the $(i, j)$-th element of the nonnegative penalization matrix $\mathbf{M}_{l}$ associated with the non-parametric component $g_{l}(\cdot)$ for $l=1, \cdots, k$.

\subsubsection{Parameter estimation}

The direct application of Newton-Raphson method in this case have some inconvenient. However, it may fixed, see for instance section 8.2 of Ortega and Rheinboldt (2000), with a damped Newton method. So, for the maximization of one of the equations in subsection 4.2.1 we follow two steps. According with Ortega et al. (2008) it is reasonable to expect that the shape parameter $\lambda$ belongs to the interval $[-3,3]$. Hence, we use it to obtain $\hat{\lambda}$ like an argument in the auxiliary function shape.parameter in the first step. Then, we find the maximum likelihood estimates $\boldsymbol{\beta}_{\hat{\lambda}}$ and $\sigma_{\hat{\lambda}}$. In this step, we have built the $\mathrm{R}$ function ssurvglg which gives us this and the related results, see chapter 6 for details about these and others $\mathrm{R}$ functions developed in this work. 


\subsubsection{Penalized score function}

For $\lambda>0$ the components of the penalized score function of $\boldsymbol{\theta}$ may be expressed as

$$
\begin{aligned}
U_{p}^{\beta_{j}} & =-\frac{1}{\lambda \sigma} \sum_{i \in D} x_{i j}\left[1-e^{\lambda \epsilon_{i}}\right]+\frac{\lambda}{\sigma \Gamma\left(\lambda^{-2}\right)} \sum_{i \in C}\left[x_{i j} \frac{a_{i}^{\lambda^{-2}} e^{-a_{i}}}{1-\mathrm{I}\left(\lambda^{-2}, a_{i}\right)}\right] \\
U_{p}^{\gamma_{j l}} & =-\frac{1}{\lambda \sigma} \sum_{i \in D} N_{i j l}\left[1-e^{\lambda \epsilon_{i}}\right]+\frac{\lambda}{\sigma \Gamma\left(\lambda^{-2}\right)} \sum_{i \in C}\left[N_{i j l} \frac{a_{i}^{\lambda^{-2}} e^{-a_{i}}}{1-\mathrm{I}\left(\lambda^{-2}, a_{i}\right)}\right]-\alpha_{l} \sum_{i=1}^{q_{l}} M_{i j l} \gamma_{i l} \\
U_{p}^{\sigma} & =-\frac{1}{\sigma} \sum_{i \in D}\left[1+\frac{1}{\lambda}\left(\epsilon_{i}-\epsilon_{i} e^{\lambda \epsilon_{i}}\right)\right]+\frac{\lambda}{\sigma \Gamma\left(\lambda^{-2}\right)} \sum_{i \in C}\left[\epsilon_{i} \frac{a_{i}^{\lambda^{-2}} e^{-a_{i}}}{1-\mathrm{I}\left(\lambda^{-2}, a_{i}\right)}\right] \text { and } \\
U_{p}^{\lambda} & =\sum_{i \in D}\left[\zeta_{\lambda}-\frac{1}{\lambda^{2}} \epsilon_{i}+\frac{2}{\lambda^{3}} e^{\lambda \epsilon_{i}}-\frac{1}{\lambda^{2}} \epsilon_{i} e^{\lambda \epsilon_{i}}\right] \\
& +\sum_{i \in C}-\frac{2 \lambda^{-3} \psi\left(\lambda^{-2}\right) I\left(\lambda^{-2}, a_{i}\right)+\frac{1}{\Gamma\left(\lambda^{-2}\right)} a_{i}^{\lambda^{-2}} e^{-a_{i}}\left(\epsilon_{i}-2 \lambda^{-1}\right)}{1-\mathrm{I}\left(\lambda^{-2}, a_{i}\right)}
\end{aligned}
$$

with $j=1, \cdots, q_{l}$ and $l=1, \cdots, k$. For $\lambda<0$ the components of the penalized score function of $\boldsymbol{\theta}$ may be expressed as

$$
\begin{aligned}
U_{p}^{\beta_{j}} & =-\frac{1}{\lambda \sigma} \sum_{i \in D} x_{i j}\left[1-e^{\lambda \epsilon_{i}}\right]+\frac{-\lambda}{\sigma \Gamma\left(\lambda^{-2}\right)} \sum_{i \in C}\left[x_{i j} \frac{a_{i}^{\lambda^{-2}} e^{-a_{i}}}{\mathrm{I}\left(\lambda^{-2}, a_{i}\right)}\right] \\
U_{p}^{\gamma_{j l}} & =-\frac{1}{\lambda \sigma} \sum_{i \in D} N_{i j l}\left[1-e^{\lambda \epsilon_{i}}\right]+\frac{-\lambda}{\sigma \Gamma\left(\lambda^{-2}\right)} \sum_{i \in C}\left[N_{i j l} \frac{a_{i}^{\lambda^{-2}} e^{-a_{i}}}{\mathrm{I}\left(\lambda^{-2}, a_{i}\right)}\right]-\alpha_{l} \sum_{i=1}^{q_{l}} M_{i j l} \gamma_{i l} \\
U_{p}^{\sigma} & =-\frac{1}{\sigma} \sum_{i \in D}\left[1+\frac{1}{\lambda}\left(\epsilon_{i}-\epsilon_{i} e^{\lambda \epsilon_{i}}\right)\right]+\frac{-\lambda}{\sigma \Gamma\left(\lambda^{-2}\right)} \sum_{i \in C} \frac{\epsilon_{i} a_{i}^{\lambda^{-2}} e^{-a_{i}}}{\mathrm{I}\left(\lambda^{-2}, a_{i}\right)} \text { and } \\
U_{p}^{\lambda} & =\sum_{i \in D}\left[\zeta_{\lambda}-\frac{1}{\lambda^{2}} \epsilon_{i}+\frac{2}{\lambda^{3}} e^{\lambda \epsilon_{i}}-\frac{1}{\lambda^{2}} \epsilon_{i} e^{\lambda \epsilon_{i}}\right] \\
& +\sum_{i \in C} \frac{2 \lambda^{-3} \psi\left(\lambda^{-2}\right) I\left(\lambda^{-2}, a_{i}\right)+\frac{1}{\Gamma\left(\lambda^{-2}\right)} a_{i}^{\lambda^{-2}} e^{-a_{i}}\left(\epsilon_{i}-2 \lambda^{-1}\right)}{\mathrm{I}\left(\lambda^{-2}, a_{i}\right)}
\end{aligned}
$$

with $j=1, \cdots, q_{l}, l=1, \cdots, k$ and $\zeta_{\lambda}=\frac{1}{\lambda}+\frac{2}{\lambda^{3}}\left\{\psi\left(\frac{1}{\lambda^{2}}\right)+2 \log (|\lambda|)-1\right\}, a_{i}=$ $\lambda^{-2} e^{\lambda \epsilon_{i}}$ and $\mathbf{D}(\mathbf{d})$ is a $(n \times n)$ diagonal matrix with $d_{i}=e^{\lambda \epsilon_{i}}$ for $i=1, \cdots, n$. 


\subsubsection{Penalized observed information matrix}

The elements of the penalized Hessian matrix for $\boldsymbol{\theta}$ in the case when $\lambda>0$ are

$$
\begin{gathered}
\frac{\partial^{2} L_{p}(\boldsymbol{\theta}, \boldsymbol{\alpha})}{\partial \beta_{l} \partial \beta_{j}}=-\frac{1}{\sigma^{2}} \sum_{i \in D} x_{i j} x_{i l} e^{\lambda \epsilon_{i}}+\sum_{i \in C} \frac{\lambda^{2} x_{i j} x_{i l} a_{i}^{\lambda^{-2}} e^{-a_{i}}}{\sigma^{2} \Gamma\left(\lambda^{-2}\right) S\left(a_{i}, \lambda\right)} b_{i}\left(\epsilon_{i}, \lambda\right) \text { for } j, l=1, \cdots, p, \\
\frac{\partial^{2} L_{p}(\boldsymbol{\theta}, \boldsymbol{\alpha})}{\partial \gamma_{j^{\prime} l} \partial \gamma_{j l}}=-\frac{1}{\sigma^{2}} \sum_{i \in D} N_{i j l} N_{i j^{\prime} l} e^{\lambda \epsilon_{i}}-\alpha_{l} M_{j j^{\prime} l}+\sum_{i \in C} \frac{\lambda^{2} N_{i j l} N_{i j^{\prime} l} a_{i}^{\lambda^{-2}} e^{-a_{i}}}{\sigma^{2} \Gamma\left(\lambda^{-2}\right) S\left(a_{i}, \lambda\right)} b_{i}\left(\epsilon_{i}, \lambda\right) \\
\quad \text { for } j, j^{\prime}=1, \cdots, q_{l} \text { and } l=1, \cdots, k,
\end{gathered}
$$$$
\frac{\partial^{2} L_{p}(\boldsymbol{\theta}, \boldsymbol{\alpha})}{\partial \gamma_{j^{\prime} l^{\prime}} \partial \gamma_{j l}}=-\frac{1}{\sigma^{2}} \sum_{i \in D} N_{i j l} N_{i j^{\prime} l^{\prime}} e^{\lambda \epsilon_{i}}+\sum_{i \in C} \frac{\lambda^{2} N_{i j l} N_{i j^{\prime} l^{\prime}} a_{i}^{\lambda^{-2}} e^{-a_{i}}}{\sigma^{2} \Gamma\left(\lambda^{-2}\right) S\left(a_{i}, \lambda\right)} b_{i}\left(\epsilon_{i}, \lambda\right)
$$$$
\text { for } j=1, \cdots, q_{l}, j^{\prime}=1, \cdots, q_{l^{\prime}} \text { and } l, l^{\prime}=1, \cdots, k \text {, but, } l \neq l^{\prime},
$$$$
\frac{\partial^{2} L_{p}(\boldsymbol{\theta}, \boldsymbol{\alpha})}{\partial \gamma_{j^{\prime} l} \partial \beta_{j}}=-\frac{1}{\sigma^{2}} \sum_{i \in D} x_{i j} N_{i j^{\prime} l} e^{\lambda \epsilon_{i}}+\sum_{i \in C} \frac{\lambda^{2} x_{i j} N_{i j^{\prime} l} a_{i}^{\lambda^{-2}} e^{-a_{i}}}{\sigma^{2} \Gamma\left(\lambda^{-2}\right) S\left(a_{i}, \lambda\right)} b_{i}\left(\epsilon_{i}, \lambda\right)
$$$$
\text { for } j=1, \cdots, p, j^{\prime}=1, \cdots, q_{l} \text { and } l=1, \cdots, k,
$$$$
\frac{\partial^{2} L_{p}(\boldsymbol{\theta}, \boldsymbol{\alpha})}{\partial \sigma^{2}}=\frac{1}{\sigma^{2}} \sum_{i \in D}\left[1+\frac{2}{\lambda} \epsilon_{i}\left(1-e^{\lambda \epsilon_{i}}\right)-\epsilon_{i}^{2} e^{\lambda \epsilon_{i}}\right]+\sum_{i \in C} \frac{\lambda \epsilon_{i}^{2} a_{i}^{\lambda^{-2}} e^{-a_{i}}}{\sigma^{2} \Gamma\left(\lambda^{-2}\right) S\left(a_{i}, \lambda\right)}\left[b_{i}\left(\epsilon_{i}, \lambda\right)-\frac{2}{\lambda \epsilon_{i}}\right],
$$$$
\frac{\partial^{2} L_{p}(\boldsymbol{\theta}, \boldsymbol{\alpha})}{\partial \sigma \partial \beta_{j}}=\frac{1}{\lambda \sigma^{2}}\left[\sum_{i \in D} x_{i j}-x_{i j} e^{\lambda \epsilon_{i}}-\lambda x_{i j} \epsilon_{i} e^{\lambda \epsilon_{i}}\right]+\sum_{i \in C} \frac{\lambda^{2} x_{i j} \epsilon_{i} a_{i}^{\lambda^{-2}} e^{-a_{i}}}{\sigma^{2} \Gamma\left(\lambda^{-2}\right) S\left(a_{i}, \lambda\right)}\left[b_{i}\left(\epsilon_{i}, \lambda\right)-\frac{1}{\lambda \epsilon_{i}}\right],
$$

for $j=1, \cdots, p$,

$$
\frac{\partial^{2} L_{p}(\boldsymbol{\theta}, \boldsymbol{\alpha})}{\partial \sigma \partial \gamma_{j l}}=\frac{1}{\lambda \sigma^{2}}\left[\sum_{i \in D} N_{i j l}-N_{i j l} e^{\lambda \epsilon_{i}}-\lambda N_{i j l} \epsilon_{i} e^{\lambda \epsilon_{i}}\right]+\sum_{i \in C} \frac{\lambda^{2} N_{i j l} \epsilon_{i} a_{i}^{\lambda^{-2}} e^{-a_{i}}}{\sigma^{2} \Gamma\left(\lambda^{-2}\right) S\left(a_{i}, \lambda\right)}\left[b_{i}\left(\epsilon_{i}, \lambda\right)-\frac{1}{\lambda}\right]
$$

for $j=1, \cdots, q_{l}$ and $l=1, \cdots, k$,

$$
\begin{aligned}
\frac{\partial L_{p}(\boldsymbol{\theta}, \boldsymbol{\alpha})}{\partial \lambda \partial \beta_{j}} & =\frac{1}{\lambda^{2} \sigma}\left[\sum_{i \in D} x_{i j}-x_{i j} e^{\lambda \epsilon_{i}}+\lambda x_{i j} \epsilon_{i} e^{\lambda \epsilon_{i}}\right] \\
& +\frac{x_{i j} a_{i}^{\lambda^{-2}} e^{-a_{i}}}{\sigma \Gamma\left(\lambda^{-2}\right) S\left(a_{i}, \lambda\right)}\left[1+\lambda^{-1}\left(\epsilon_{i}-2 \lambda^{-1}\left(\log a_{i}+1\right)\right)+\lambda a_{i}\left(2 \lambda^{-1}-\epsilon_{i}\right)\right. \\
& \left.+2 \lambda^{-2} \psi\left(\lambda^{-2}\right)+\frac{\lambda}{S\left(a_{i}, \lambda\right)}\left\{2 \lambda^{-3} \psi\left(\lambda^{-2}\right) I\left(\lambda^{-2}, a_{i}\right)+\frac{1}{\Gamma\left(\lambda^{-2}\right)} a_{i}^{\lambda^{-2}} e^{-a_{i}}\left(\epsilon_{i}-2 \lambda^{-1}\right)\right\}\right],
\end{aligned}
$$

for $j=1, \cdots, p$, 
and

$$
\begin{aligned}
& \frac{\partial L_{p}(\boldsymbol{\theta}, \boldsymbol{\alpha})}{\partial \lambda \partial \gamma_{j l}}=\frac{1}{\lambda^{2} \sigma}\left[\sum_{i \in D} N_{i j l}-N_{i j l} e^{\lambda \epsilon_{i}}+\lambda N_{i j l} \epsilon_{i} e^{\lambda \epsilon_{i}}\right] \\
& +\frac{N_{i j l} a_{i}^{\lambda^{-2}} e^{-a_{i}}}{\sigma \Gamma\left(\lambda^{-2}\right) S\left(a_{i}, \lambda\right)}\left[1+\lambda^{-1}\left(\epsilon_{i}-2 \lambda^{-1}\left(\log a_{i}+1\right)\right)+\lambda a_{i}\left(2 \lambda^{-1}-\epsilon_{i}\right)+2 \lambda^{-2} \psi\left(\lambda^{-2}\right)\right. \\
& \left.+\frac{\lambda}{S\left(a_{i}, \lambda\right)}\left(2 \lambda^{-3} \psi\left(\lambda^{-2}\right) I\left(\lambda^{-2}, a_{i}\right)+\frac{1}{\Gamma\left(\lambda^{-2}\right)} a_{i}^{\lambda^{-2}} e^{-a_{i}}\left(\epsilon_{i}-2 \lambda^{-1}\right)\right)\right], \\
& \text { for } j=1, \cdots, q_{l} \text { and } l=1, \cdots, k \text {, } \\
& \frac{\partial L_{p}(\boldsymbol{\theta}, \boldsymbol{\alpha})}{\partial \lambda \partial \sigma}=\frac{1}{\lambda^{2} \sigma} \sum_{i \in D}\left[\epsilon_{i}-\frac{1}{\lambda^{2} \sigma} \epsilon_{i} e^{\lambda \epsilon_{i}}+\frac{1}{\lambda \sigma} \epsilon_{i}^{2} e^{\lambda \epsilon_{i}}\right] \\
& +\frac{\epsilon_{i} a_{i}^{\lambda^{-2}} e^{-a_{i}}}{\sigma \Gamma\left(\lambda^{-2}\right) S\left(a_{i}, \lambda\right)}\left[1+\lambda^{-1}\left(\epsilon_{i}-2 \lambda^{-1}\left(\log a_{i}+1\right)\right)+\lambda a_{i}\left(2 \lambda^{-1}-\epsilon_{i}\right)+2 \lambda^{-2} \psi\left(\lambda^{-2}\right)\right. \\
& \left.+\frac{\lambda}{S\left(a_{i}, \lambda\right)}\left(2 \lambda^{-3} \psi\left(\lambda^{-2}\right) I\left(\lambda^{-2}, a_{i}\right)+\frac{1}{\Gamma\left(\lambda^{-2}\right)} a_{i}^{\lambda^{-2}} e^{-a_{i}}\left(\epsilon_{i}-2 \lambda^{-1}\right)\right)\right] \text { and } \\
& \frac{\partial^{2} L_{p}(\boldsymbol{\theta}, \boldsymbol{\alpha})}{\partial \lambda^{2}}=\sum_{i \in D}\left[-\frac{1}{\lambda^{2}} \tau_{\lambda}+\frac{2}{\lambda^{3}} \epsilon_{i}-\frac{6}{\lambda^{4}} e^{\lambda \epsilon_{i}}+\frac{4}{\lambda^{3}} \epsilon_{i} e^{\lambda \epsilon_{i}}-\frac{1}{\lambda^{2}} \epsilon_{i}^{2} e^{\lambda \epsilon_{i}}\right] \\
& -\sum_{i \in C}\left[6 \lambda^{-4} \psi\left(\lambda^{-2}\right) I\left(\lambda^{-2}, a_{i}\right)+4 \lambda^{-6} \psi^{\prime}\left(\lambda^{-2}\right) I\left(\lambda^{-2}, a_{i}\right)\right. \\
& -2 \lambda^{-3} \psi\left(\lambda^{-2}\right)\left[2 \lambda^{-3} \psi\left(\lambda^{-2}\right) I\left(\lambda^{-2}, a_{i}\right)+\frac{1}{\Gamma\left(\lambda^{-2}\right)} a_{i}^{\lambda^{-2}} e^{-a_{i}}\left(\epsilon_{i}-2 \lambda^{-1}\right)\right] \\
& +a_{i}^{\lambda^{-2}} e^{-a_{i}}\left[\frac{2}{\Gamma\left(\lambda^{-2}\right)} \lambda^{-3} \psi\left(\lambda^{-2}\right)\left(\epsilon_{i}-2 \lambda^{-1}\right)\right. \\
& +\frac{\lambda^{-2}}{\Gamma\left(\lambda^{-2}\right)}\left(\epsilon_{i}-2 \lambda^{-1}\left(\log a_{i}+1\right)\right)\left(\epsilon_{i}-2 \lambda^{-1}\right) \\
& \left.\left.-\frac{a_{i}}{\Gamma\left(\lambda^{-2}\right)}\left(\epsilon_{i}-2 \lambda^{-1}\right)^{2}+\frac{2 \lambda^{-2}}{\Gamma\left(\lambda^{-2}\right)}\right]\right] \frac{1}{S\left(a_{i}, \lambda\right)} \\
& -\sum_{i \in C}\left[2 \lambda^{-3} \psi\left(\lambda^{-2}\right) I\left(\lambda^{-2}, a_{i}\right)+\frac{1}{\Gamma\left(\lambda^{-2}\right)} a_{i}^{\lambda^{-2}} e^{-a_{i}}\left(\epsilon_{i}-2 \lambda^{-1}\right)\right]^{2} \frac{1}{S\left(a_{i}, \lambda\right)^{2}} .
\end{aligned}
$$


The elements of the penalized Hessian matrix for $\boldsymbol{\theta}$ in the case when $\lambda<0$ are

$$
\begin{aligned}
\frac{\partial^{2} L_{p}(\boldsymbol{\theta}, \boldsymbol{\alpha})}{\partial \beta_{l} \partial \beta_{j}} & =-\frac{1}{\sigma^{2}} \sum_{i \in D} x_{i j} x_{i l} e^{\lambda \epsilon_{i}}+\sum_{i \in C}-\frac{\lambda^{2} x_{i j} x_{i l} a_{i}^{\lambda^{-2}} e^{-a_{i}}}{\sigma^{2} \Gamma\left(\lambda^{-2}\right) \mathrm{I}\left(a_{i}, \lambda\right)} b_{i}\left(\epsilon_{i}, \lambda\right) \text { for } j, l=1, \cdots, p, \\
\frac{\partial^{2} L_{p}(\boldsymbol{\theta}, \boldsymbol{\alpha})}{\partial \gamma_{j^{\prime} l} \partial \gamma_{j l}} & =-\frac{1}{\sigma^{2}} \sum_{i \in D} N_{i j l} N_{i j^{\prime} l} e^{\lambda \epsilon_{i}}-\alpha_{l} M_{j j^{\prime} l}+\sum_{i \in C}-\frac{\lambda^{2} N_{i j l} N_{i j^{\prime} l} a_{i}^{\lambda^{-2}} e^{-a_{i}}}{\sigma^{2} \Gamma\left(\lambda^{-2}\right) \mathrm{I}\left(a_{i}, \lambda\right)} b_{i}\left(\epsilon_{i}, \lambda\right) \\
& \text { for } j, j^{\prime}=1, \cdots, q_{l} \text { and } l=1, \cdots, k,
\end{aligned}
$$$$
\frac{\partial^{2} L_{p}(\boldsymbol{\theta}, \boldsymbol{\alpha})}{\partial \gamma_{j^{\prime} l^{\prime}} \partial \gamma_{j l}}=-\frac{1}{\sigma^{2}} \sum_{i \in D} N_{i j l} N_{i j^{\prime} l^{\prime}} e^{\lambda \epsilon_{i}}+\sum_{i \in C}-\frac{\lambda^{2} N_{i j l} N_{i j^{\prime} l^{\prime}} a_{i}^{\lambda^{-2}} e^{-a_{i}}}{\sigma^{2} \Gamma\left(\lambda^{-2}\right) \mathrm{I}\left(a_{i}, \lambda\right)} b_{i}\left(\epsilon_{i}, \lambda\right)
$$$$
\text { for } j=1, \cdots, q_{l}, j^{\prime}=1, \cdots, q_{l^{\prime}} \text { and } l, l^{\prime}=1, \cdots, k \text {, but, } l \neq l^{\prime} \text {, }
$$$$
\frac{\partial^{2} L_{p}(\boldsymbol{\theta}, \boldsymbol{\alpha})}{\partial \gamma_{j^{\prime} l} \partial \beta_{j}}=-\frac{1}{\sigma^{2}} \sum_{i \in D} x_{i j} N_{i j^{\prime} l} e^{\lambda \epsilon_{i}}+\sum_{i \in C}-\frac{\lambda^{2} x_{i j} N_{i j^{\prime} l} a_{i}^{\lambda^{-2}} e^{-a_{i}}}{\sigma^{2} \Gamma\left(\lambda^{-2}\right) \mathrm{I}\left(a_{i}, \lambda\right)} b_{i}\left(\epsilon_{i}, \lambda\right)
$$$$
\text { for } j=1, \cdots, p, j^{\prime}=1, \cdots, q_{l} \text { and } l=1, \cdots, k \text {, }
$$$$
\frac{\partial^{2} L_{p}(\boldsymbol{\theta}, \boldsymbol{\alpha})}{\partial \sigma^{2}}=\frac{1}{\sigma^{2}} \sum_{i \in D}\left[1+\frac{2}{\lambda} \epsilon_{i}\left(1-e^{\lambda \epsilon_{i}}\right)-\epsilon_{i}^{2} e^{\lambda \epsilon_{i}}\right]+\sum_{i \in C} \frac{\lambda \epsilon_{i}^{2} a_{i}^{\lambda^{-2}} e^{-a_{i}}}{\sigma^{2} \Gamma\left(\lambda^{-2}\right) \mathrm{I}\left(a_{i}, \lambda\right)}\left[b_{i}\left(\epsilon_{i}, \lambda\right)-\frac{2}{\lambda \epsilon_{i}}\right],
$$$$
\frac{\partial^{2} L_{p}(\boldsymbol{\theta}, \boldsymbol{\alpha})}{\partial \sigma \partial \beta_{j}}=\frac{1}{\lambda \sigma^{2}}\left[\sum_{i \in D} x_{i j}-x_{i j} e^{\lambda \epsilon_{i}}-\lambda x_{i j} \epsilon_{i} e^{\lambda \epsilon_{i}}\right]+\sum_{i \in C} \frac{\lambda^{2} x_{i j} \epsilon_{i} a_{i}^{\lambda^{-2}} e^{-a_{i}}}{\sigma^{2} \Gamma\left(\lambda^{-2}\right) \mathrm{I}\left(a_{i}, \lambda\right)}\left[b_{i}\left(\epsilon_{i}, \lambda\right)-\frac{1}{\lambda \epsilon_{i}}\right] \text {, }
$$

$$
\begin{gathered}
\text { for } j=1, \cdots, p, \\
\frac{\partial^{2} L_{p}(\boldsymbol{\theta}, \boldsymbol{\alpha})}{\partial \sigma \partial \gamma_{j l}}=\frac{1}{\lambda \sigma^{2}}\left[\sum_{i \in D} N_{i j l}-N_{i j l} e^{\lambda \epsilon_{i}}-\lambda N_{i j l} \epsilon_{i} e^{\lambda \epsilon_{i}}\right]+\sum_{i \in C} \frac{\lambda^{2} N_{i j l} \epsilon_{i} a_{i}^{\lambda^{-2}} e^{-a_{i}}}{\sigma^{2} \Gamma\left(\lambda^{-2}\right) \mathrm{I}\left(a_{i}, \lambda\right)}\left[b_{i}\left(\epsilon_{i}, \lambda\right)-\frac{1}{\lambda}\right],
\end{gathered}
$$$$
\text { for } j=1, \cdots, q_{l} \text { and } l=1, \cdots, k \text {, }
$$

$$
\begin{aligned}
\frac{\partial L_{p}(\boldsymbol{\theta}, \boldsymbol{\alpha})}{\partial \lambda \partial \beta_{j}} & =\frac{1}{\lambda^{2} \sigma}\left[\sum_{i \in D} x_{i j}-x_{i j} e^{\lambda \epsilon_{i}}+\lambda x_{i j} \epsilon_{i} e^{\lambda \epsilon_{i}}\right] \\
& +\frac{x_{i j} a_{i}^{\lambda^{-2}} e^{-a_{i}}}{\sigma \Gamma\left(\lambda^{-2}\right) \mathrm{I}\left(a_{i}, \lambda\right)}\left[1+\lambda^{-1}\left(\epsilon_{i}-2 \lambda^{-1}\left(\log a_{i}+1\right)\right)+\lambda a_{i}\left(2 \lambda^{-1}-\epsilon_{i}\right)\right. \\
& \left.+2 \lambda^{-2} \psi\left(\lambda^{-2}\right)+\frac{\lambda}{\mathrm{I}\left(a_{i}, \lambda\right)}\left(2 \lambda^{-3} \psi\left(\lambda^{-2}\right) I\left(\lambda^{-2}, a_{i}\right)+\frac{1}{\Gamma\left(\lambda^{-2}\right)} a_{i}^{\lambda^{-2}} e^{-a_{i}}\left(\epsilon_{i}-2 \lambda^{-1}\right)\right)\right],
\end{aligned}
$$

for $j=1, \cdots, p$, 
and

$$
\begin{aligned}
\frac{\partial L_{p}(\boldsymbol{\theta}, \boldsymbol{\alpha})}{\partial \lambda \partial \gamma_{j l}} & =\frac{1}{\lambda^{2} \sigma}\left[\sum_{i \in D} N_{i j l}-N_{i j l} e^{\lambda \epsilon_{i}}+\lambda N_{i j l} \epsilon_{i} e^{\lambda \epsilon_{i}}\right] \\
& +\frac{N_{i j l} a_{i}^{\lambda^{-2}} e^{-a_{i}}}{\sigma \Gamma\left(\lambda^{-2}\right) \mathrm{I}\left(a_{i}, \lambda\right)}\left[1+\lambda^{-1}\left(\epsilon_{i}-2 \lambda^{-1}\left(\log a_{i}+1\right)\right)+\lambda a_{i}\left(2 \lambda^{-1}-\epsilon_{i}\right)+2 \lambda^{-2} \psi\left(\lambda^{-2}\right)\right. \\
& \left.+\frac{\lambda}{S\left(a_{i}, \lambda\right)}\left(2 \lambda^{-3} \psi\left(\lambda^{-2}\right) I\left(\lambda^{-2}, a_{i}\right)+\frac{1}{\Gamma\left(\lambda^{-2}\right)} a_{i}^{\lambda^{-2}} e^{-a_{i}}\left(\epsilon_{i}-2 \lambda^{-1}\right)\right)\right]
\end{aligned}
$$

for $j=1, \cdots, q_{l}$ and $l=1, \cdots, k$,

$$
\begin{aligned}
\frac{\partial L_{p}(\boldsymbol{\theta}, \boldsymbol{\alpha})}{\partial \lambda \partial \sigma} & =\frac{1}{\lambda^{2} \sigma} \sum_{i \in D}\left[\epsilon_{i}-\frac{1}{\lambda^{2} \sigma} \epsilon_{i} e^{\lambda \epsilon_{i}}+\frac{1}{\lambda \sigma} \epsilon_{i}^{2} e^{\lambda \epsilon_{i}}\right] \\
& +\frac{\epsilon_{i} a_{i}^{\lambda^{-2}} e^{-a_{i}}}{\sigma \Gamma\left(\lambda^{-2}\right) \mathrm{I}\left(a_{i}, \lambda\right)}\left[1+\lambda^{-1}\left(\epsilon_{i}-2 \lambda^{-1}\left(\log a_{i}+1\right)\right)+\lambda a_{i}\left(2 \lambda^{-1}-\epsilon_{i}\right)+2 \lambda^{-2} \psi\left(\lambda^{-2}\right)\right. \\
& \left.+\frac{\lambda}{\mathrm{I}\left(a_{i}, \lambda\right)}\left(2 \lambda^{-3} \psi\left(\lambda^{-2}\right) \mathrm{I}\left(\lambda^{-2}, a_{i}\right)+\frac{1}{\Gamma\left(\lambda^{-2}\right)} a_{i}^{\lambda^{-2}} e^{-a_{i}}\left(\epsilon_{i}-2 \lambda^{-1}\right)\right)\right] \text { and } \\
\frac{\partial^{2} L_{p}(\boldsymbol{\theta}, \boldsymbol{\alpha})}{\partial \lambda^{2}} & =\sum_{i \in D}\left[-\frac{1}{\lambda^{2}} \tau_{\lambda}+\frac{2}{\lambda^{3}} \epsilon_{i}-\frac{6}{\lambda^{4}} e^{\lambda \epsilon_{i}}+\frac{4}{\lambda^{3}} \epsilon_{i} e^{\lambda \epsilon_{i}}-\frac{1}{\lambda^{2}} \epsilon_{i}^{2} e^{\lambda \epsilon_{i}}\right] \\
& -\sum_{i \in C}\left[6 \lambda^{-4} \psi\left(\lambda^{-2}\right) \mathrm{I}\left(\lambda^{-2}, a_{i}\right)+4 \lambda^{-6} \psi^{\prime}\left(\lambda^{-2}\right) \mathrm{I}\left(\lambda^{-2}, a_{i}\right)\right. \\
& -2 \lambda^{-3} \psi\left(\lambda^{-2}\right)\left[2 \lambda^{-3} \psi\left(\lambda^{-2}\right) \mathrm{I}\left(\lambda^{-2}, a_{i}\right)+\frac{1}{\Gamma\left(\lambda^{-2}\right)} a_{i}^{\lambda^{-2}} e^{-a_{i}}\left(\epsilon_{i}-2 \lambda^{-1}\right)\right] \\
& +a_{i}^{\lambda^{-2}} e^{-a_{i}}\left[\frac{2}{\Gamma\left(\lambda^{-2}\right)} \lambda^{-3} \psi\left(\lambda^{-2}\right)\left(\epsilon_{i}-2 \lambda^{-1}\right)\right. \\
& +\frac{\lambda^{-2}}{\Gamma\left(\lambda^{-2}\right)}\left(\epsilon_{i}-2 \lambda^{-1}\left(\log a_{i}+1\right)\right)\left(\epsilon_{i}-2 \lambda^{-1}\right) \\
& \left.\left.-\frac{a_{i}}{\Gamma\left(\lambda^{-2}\right)}\left(\epsilon_{i}-2 \lambda^{-1}\right)^{2}+\frac{2 \lambda^{-2}}{\Gamma\left(\lambda^{-2}\right)}\right]\right] \frac{1}{\mathrm{I}\left(a_{i}, \lambda\right)} \\
& -\sum_{i \in C}\left[2 \lambda^{-3} \psi\left(\lambda^{-2}\right) I\left(\lambda^{-2}, a_{i}\right)+\frac{1}{\Gamma\left(\lambda^{-2}\right)} a_{i}^{\lambda^{-2}} e^{-a_{i}}\left(\epsilon_{i}-2 \lambda^{-1}\right)\right]^{2} \frac{1}{\mathrm{I}\left(a_{i}, \lambda\right)^{2}} \\
&
\end{aligned}
$$


with

$$
b_{i}\left(\epsilon_{i}, \lambda\right)=a_{i}-\lambda^{-2}-\frac{a_{i}^{\lambda^{-2}} e^{-a_{i}}}{\Gamma\left(\lambda^{-2}\right) \mathrm{S}\left(a_{i}, \lambda\right)},
$$

$S\left(a_{i}, \lambda\right)=1-\mathrm{I}\left(\lambda^{-2}, a_{i}\right), a_{i}=\lambda^{-2} e^{\lambda \epsilon_{i}}$ and $j=1, \cdots, n$.

\subsection{Effective degrees of freedom}

Similarly to the uncensored case, we may define an approximation of the degree of freedom by analogy with the linear case. We obtain

$$
\begin{aligned}
\operatorname{df}(\boldsymbol{\alpha}) & =\operatorname{tr}\left[\tilde{\mathbf{X}}\left(\tilde{\mathbf{X}}^{\top} \tilde{\mathbf{X}}+\sigma^{2} \mathbf{M}_{\alpha}\right)^{-1} \tilde{\mathbf{X}}^{\top}\right] \\
& =\operatorname{tr}\left[\left(\mathbf{I}+\sigma^{2} \mathbf{Q}^{-\frac{1}{2}} \mathbf{M}_{\alpha} \mathbf{Q}^{-\frac{1}{2}}\right)^{-1}\right] \\
& =p+\sum_{l=p+1}^{\operatorname{dim}\left(\mathbf{M}_{\alpha}\right)} \frac{1}{1+\phi_{l}},
\end{aligned}
$$

where $\tilde{\mathbf{X}}=\left(\mathbf{X}^{\top}, \mathbf{N}_{1}^{\top}, \cdots, \mathbf{N}_{k}^{\top}\right)^{\top}, \mathbf{M}_{\alpha}=\operatorname{diag}\left(\mathbf{0}_{p p}, \alpha_{1} \mathbf{M}_{1}, \cdots, \alpha_{k} \mathbf{M}_{k}\right), \phi_{l}$ are the eigenvalues of the matrix $\sigma^{2} \mathbf{Q}^{-\frac{1}{2}} \mathbf{M}_{\alpha} \mathbf{Q}^{-\frac{1}{2}}$ and $\mathbf{0}_{p p}$ is a $(p \times p)$ matrix of zeros. Additionally,

$$
\operatorname{dim}\left(\mathbf{M}_{\alpha}\right)>\operatorname{df}(\alpha)>p+\sum_{j=1}^{k} \text { zero }_{j}
$$

where $z e r o_{j}$ is the number of eigenvalues zero of $\mathbf{M}_{j}$. Therefore, one has $\operatorname{df}(\alpha)+2$ parameters to be estimated from the estimating iterative process.

\subsection{Smoothing parameter}

Up to now the smoothing parameters $\alpha_{j}, j=1, \cdots, k$, have been fixed, but in real situations they should be estimated. In the chapter 3 was explained the drawbacks if we want to apply a multivariate version of the generalized crossvalidation $\operatorname{GCV}(\cdot)$ method. So, we minimize the information measure $\operatorname{AIC}(\boldsymbol{\alpha})$ or $\operatorname{SIC}(\boldsymbol{\alpha})$. These criteria guarantee a compromise between model complexity and goodness-of-fit, which is the main goal according to Hastie and Tibshirani (1990); Rigby and Stasinopoulos (2006); Vanegas and Paula (2016) and Wood (2006). 


\subsection{Model selection}

For model selection we may apply the Akaike or Schwarz Information Criterion Akaike (1974); Schwarz (1978), respectively, which are defined as

$$
\operatorname{AIC}(\boldsymbol{\alpha})=-2 L_{p}(\boldsymbol{\theta}, \alpha)+2[2+\operatorname{df}(\boldsymbol{\alpha})]
$$

and

$$
\operatorname{SIC}(\boldsymbol{\alpha})=-2 L_{p}(\boldsymbol{\theta}, \alpha)+\log (n)[2+\mathrm{df}(\boldsymbol{\alpha})]
$$

We choose the model with the smallest AIC or SIC value.

\subsection{Asymptotic statements}

To study the limit behavior of the penalized maximum likelihood estimators $\hat{\boldsymbol{\theta}}$ under censoring, we adopt the approach of Vanegas and Paula (2017) and the assumptions of regularity given in Bagdonavicius and Nikulin (2001). This approach consists in assuming the number of knots fixed, which means that the penalty matrices and the sizes of $\gamma_{1}, \cdots, \gamma_{k}$ are not functions of the sample size $n$. Some additional conditions are necessary to guarantee the consistency and asymptotic normality of the estimators, given by

1. There is $\boldsymbol{\gamma}_{j}^{0} \in \mathbf{R}^{q_{j}-1}$ such that $\mathbf{g}_{j}(t)=\mathbf{N}_{j}^{\top}(t) \boldsymbol{\gamma}_{j}^{0}$ for all $t \in, j=1, \cdots, k$. Hence, $\gamma^{0}=\left(\left(\gamma_{1}^{0}\right)^{\top}, \cdots,\left(\gamma_{k}^{0}\right)^{\top}\right)^{\top}$ is the true parameter vector;

2. The smoothing parameter may dependent on the sample size. For a sample of size $n$, let $\alpha_{n}$ be the smoothing parameter. So, $\frac{1}{n} \alpha_{n} \rightarrow 0$ when $n \rightarrow \infty$

3. If the regularity conditions are satisfied we may ensure that

(i) $n^{-1} \mathbf{U}_{n}\left(\boldsymbol{\theta}_{0}\right) \stackrel{P}{\longrightarrow} \mathbf{0}$ where $\boldsymbol{\theta}_{0}=\left(\left(\boldsymbol{\beta}^{0}\right)^{\top},\left(\boldsymbol{\gamma}^{0}\right)^{\top}, \sigma^{0}\right)^{\top}$ is the true parameter vector for $\boldsymbol{\theta}$; 
(ii) $n^{-1} \mathbf{J}_{n}\left(\boldsymbol{\theta}_{0}\right) \rightarrow \Sigma\left(\boldsymbol{\theta}_{0}\right)$;

(iii) $n^{-1} \mathbf{J}_{n}\left(\boldsymbol{\theta}^{*}\right) \rightarrow \Sigma\left(\boldsymbol{\theta}_{0}\right)$ for all $\boldsymbol{\theta}^{*} \in\left[\hat{\boldsymbol{\theta}}, \boldsymbol{\theta}_{0}\right]$;

(iv) $\mathbf{J}_{n}^{-\frac{1}{2}}\left(\boldsymbol{\theta}_{0}\right) \mathbf{U}_{n}\left(\boldsymbol{\theta}_{0}\right) \stackrel{D}{\longrightarrow} \boldsymbol{N}(\mathbf{0}, \mathbf{I})$;

(v) $P\left[\frac{1}{n}\left|\frac{\partial \mathbf{J}_{n}(\theta)_{i j}}{\partial \theta_{l}}\right|_{\boldsymbol{\theta}=\boldsymbol{\theta}^{*}} \mid<\phi\right] \stackrel{P}{\longrightarrow} 1$, for all $(i, j, l)$ and for all $\boldsymbol{\theta}^{*} \in\left[\hat{\boldsymbol{\theta}}, \boldsymbol{\theta}_{0}\right]$,

where $\phi$ is a constant and $\mathbf{J}_{n}(\boldsymbol{\theta})_{i j}$ is the $(i, j)$-th element of $\mathbf{J}_{n}(\boldsymbol{\theta})$.

In addition

$$
\begin{aligned}
\mathbf{U}_{p}^{\boldsymbol{\theta}}\left(\boldsymbol{\theta}_{0}\right) & =\mathbf{U}_{n}\left(\boldsymbol{\theta}_{0}\right)-\tilde{\mathbf{M}}_{\boldsymbol{\alpha}}, \\
\mathbf{J}_{p}^{\boldsymbol{\theta} \theta}\left(\boldsymbol{\theta}_{0}\right) & =\mathbf{J}_{n}\left(\boldsymbol{\theta}_{0}\right)+\tilde{\mathbf{M}}_{\boldsymbol{\alpha},},
\end{aligned}
$$

where $\mathbf{J}_{n}\left(\boldsymbol{\theta}_{0}\right)=-\left.\frac{\partial^{2} L(\boldsymbol{\theta})}{\partial \boldsymbol{\theta} \boldsymbol{\theta} \boldsymbol{\theta}}\right|_{\boldsymbol{\theta}=\boldsymbol{\theta}_{0}}, \tilde{\mathbf{M}}_{\boldsymbol{\alpha}}=\operatorname{diag}\left(\mathbf{M}_{\boldsymbol{\alpha}}, \mathbf{0}_{1}\right)$ and $\left[\hat{\boldsymbol{\theta}}, \boldsymbol{\theta}_{0}\right]$ denotes the segment of line joining $\hat{\boldsymbol{\theta}}$ and $\boldsymbol{\theta}_{0}$.

Theorem. Under the conditions (1) - (3) we may prove that $\hat{\boldsymbol{\theta}}$ is a consistent estimator of $\boldsymbol{\theta}_{0}$, and

$$
\left[\mathbf{J}_{n}\left(\boldsymbol{\theta}_{0}\right)\right]^{-\frac{1}{2}}\left[\mathbf{J}_{n}\left(\boldsymbol{\theta}_{0}\right)+\tilde{\mathbf{M}}_{\boldsymbol{\alpha}}\right]\left(\hat{\boldsymbol{\theta}}-\boldsymbol{\theta}_{0}\right) \stackrel{D}{\longrightarrow} \boldsymbol{N}(0, \mathbf{I}) .
$$

The proof is quite analogous to the one given in appendix of Vanegas and Paula (2017). Hence, the asymptotic variance-covariance matrix of $\hat{\boldsymbol{\theta}}$ takes the form

$$
\operatorname{Var}(\hat{\boldsymbol{\theta}})=\left[\mathbf{J}_{n}(\boldsymbol{\theta})+\tilde{\mathbf{M}}_{\boldsymbol{\alpha}}\right]^{-1}\left[\mathbf{J}_{n}(\boldsymbol{\theta})\right]\left[\mathbf{J}_{n}(\boldsymbol{\theta})+\tilde{\mathbf{M}}_{\boldsymbol{\alpha}}\right]^{-1} .
$$

A consistent estimator of $\operatorname{Var}(\hat{\boldsymbol{\theta}})$ is $\operatorname{Var}(\hat{\boldsymbol{\theta}})$ evaluated at $\boldsymbol{\theta}$ estimate. 


\section{Chapter 5}

\section{Diagnostic analysis}

An important step in regression analysis is to apply diagnostic methods, and an efficient form to evaluate several deficiencies with respect to the fitted model is the residual analysis. A residual analysis allow us to detect the presence of outlying observations as well as departures from the error assumptions. Other aspect of the diagnostic method is the influence analysis which allow us to evaluate the behavior of the estimates under some perturbation in the model or data. In the first section we will present deviance-type residuals, quantile residuals and an overall goodness-of-fit criterion. In the second section we will derive the normal curvature of local influence under some usual perturbation schemes.

\subsection{Residual analysis}

\subsubsection{Deviance residual}

The deviance is a usual measure of goodness-of-fit often used in generalized linear models, see for instance McCullagh and Nelder (1989), derived from the deviance $D(\mathbf{y} ; \hat{\boldsymbol{\mu}})=\sum_{i} d_{i}^{2}$, where $d_{i}=\operatorname{sign}\left(y_{i}-\hat{\mu}_{i}\right) \sqrt{2}\left\{l_{i}\left(y_{i} ; \tilde{\mu}\right)-l_{i}\left(y_{i} ; \hat{\mu}\right)\right\}^{\frac{1}{2}}$ with $l_{i}\left(y_{i} ; \cdot\right)$ being the contribution of $y_{i}$ to the total log-likelihood function, $\tilde{\mu}$ denotes the maximum likelihood estimate of $\mu$ based only on $y_{i}$ and $\hat{\mu}$ is the maximum likelihood estimate of $\mu$ under the fitted model. In addition, $\operatorname{sign}\left(y_{i}-\hat{\mu}_{i}\right)$ denotes the sign of $y_{i}-\hat{\mu}_{i}$, for $i=1, \cdots, n$. 


\subsubsection{Uncensored case}

In this case, we are working with the following deviance residual:

$$
d_{i}=\operatorname{sign}\left(y_{i}-\hat{\mu}_{i}\right) \sqrt{2}\left(\frac{1}{\hat{\lambda}^{2}} e^{\hat{\lambda}_{i}}-\frac{1}{\hat{\lambda}} \hat{\epsilon}_{i}-\frac{1}{\hat{\lambda}^{2}}\right)^{\frac{1}{2}},
$$

where $\hat{\epsilon}_{i}=\frac{y_{i}-\mathbf{x}_{i}^{\top} \hat{\beta}-\mathbf{N}_{i 1}^{\top} \hat{\gamma}_{1}-\cdots \mathbf{N}_{i k}^{\top} \hat{\gamma}_{k}}{\hat{\sigma}}$, for $i=1, \cdots, n$.

\subsubsection{Censored case}

Ortega et al. (2008) studied three types of deviance residuals for generalized log-gamma linear regression models. In general, the resulting behavior of the empirical distributions of the three residuals, after intensive Monte Carlo studies, appear to be approximately symmetric around zero. We select the following residuals:

when $\lambda>0$,

$d_{i}=\operatorname{sign}\left(y_{i}-\hat{\mu}_{i}\right) \sqrt{2}\left\{\left(1-\delta_{i}\right)\left(\frac{1}{\hat{\lambda}^{2}} e^{\hat{\lambda} \hat{\epsilon}_{i}}-\frac{1}{\hat{\lambda}} \hat{\epsilon}_{i}-\frac{1}{\hat{\lambda}^{2}}\right)^{\frac{1}{2}}+\delta_{i}\left(-\log \left(1-F_{\epsilon}\left(y_{i} ; \hat{\boldsymbol{\theta}}\right)\right)\right)^{\frac{1}{2}}\right\}$,

when $\lambda<0$,

$$
d_{i}=\operatorname{sign}\left(y_{i}-\hat{\mu}_{i}\right) \sqrt{2}\left\{\left(1-\delta_{i}\right)\left(\frac{1}{\hat{\lambda}^{2}} e^{\hat{\hat{\lambda}} \hat{\epsilon}_{i}}-\frac{1}{\hat{\lambda}} \hat{\epsilon}_{i}-\frac{1}{\hat{\lambda}^{2}}\right)^{\frac{1}{2}}+\delta_{i}\left(-\log \left(F_{\epsilon}\left(y_{i} ; \hat{\boldsymbol{\theta}}\right)\right)\right)^{\frac{1}{2}}\right\},
$$

where $F_{\epsilon}\left(y_{i} ; \boldsymbol{\theta}\right)$ is the cumulative distribution function of a generalized loggamma variable, $\delta_{i}$ denotes the censoring indicator, 0 for uncensored observations and 1 for censored observations.

\subsubsection{Overall goodness-of-fit}

Vanegas and Paula (2016) proposed a criterion to evaluate the overall goodnessof-fit of the fitted models. This graphical criterion is based on quantile residuals and it is possible to summarize it by the statistic $\Upsilon$ defined below. Under the uncensored case, one has

$$
\Upsilon=\frac{1}{n} \sum_{j=1}^{n}\left|\Phi^{-1}\left[F_{\epsilon}\left(\hat{\epsilon}^{(j)} ; \hat{\boldsymbol{\theta}}\right)\right]-v^{(j)}\right|,
$$


where $\hat{\epsilon}_{(j)}$ is the $j$-th order statistic of $\hat{\epsilon}_{1}, \cdots, \hat{\epsilon}_{n}$ and $v^{j}$ is the expectation of the $j$-th order statistic in a sample of size $n$ from the standard normal distribution. Under the censored case we obtain

$$
\Upsilon=\frac{\sum_{j=1}^{n}\left(1-\delta_{i}\right)\left|\Phi^{-1}\left[\bar{F}_{\epsilon}\left(\hat{\epsilon}^{(j)} ; \hat{\boldsymbol{\theta}}\right)\right]-\Phi^{-1}\left[\hat{F}_{\epsilon}\left(\hat{\epsilon}^{(j)} ; \hat{\boldsymbol{\theta}}\right)\right]\right|}{\sum_{j=1}^{n}\left(1-\delta_{i}\right)},
$$

where $\bar{F}_{\epsilon}\left(\hat{\epsilon}^{(j)} ; \hat{\boldsymbol{\theta}}\right)$ denotes the cumulative distribution function of $\epsilon$ estimated from $\left(\hat{\epsilon}_{1}, \delta_{1}\right), \cdots,\left(\hat{\epsilon}_{n}, \delta_{n}\right)$ through the Kaplan-Meier estimator (see, for instance, Colosimo and Giolo (2006)).

So, small values of $\Upsilon$ indicate that the fitted model describes adequately the data. Visually, an acceptable goodness-of-fit is when the difference between the normal QQ-plot of the overall residuals and the straight line with zero intercept and unit slope is small.

\subsection{Local influence}

A key reason behind of diagnostic methods is to answer the following natural and important question: how the parameter estimates change under perturbations in the model/data? One quite original approach to this question was given by Cook (1986). Through the perturbed log-likelihood function Cook assessed the influence of small perturbations in the model/data on the parameter estimates. So, a reasonable extension to semi-parametric models is to take the following penalized log-likelihood function:

$$
L_{p}(\boldsymbol{\theta} \mid \boldsymbol{\omega}, \boldsymbol{\alpha})=L(\boldsymbol{\theta} \mid \boldsymbol{\omega})-\frac{\alpha_{1}}{2} \boldsymbol{\gamma}_{1}^{\top} \mathbf{M}_{1} \boldsymbol{\gamma}_{1}-\cdots-\frac{\alpha_{k}}{2} \boldsymbol{\gamma}_{k}^{\top} \mathbf{M}_{k} \boldsymbol{\gamma}_{k}
$$

which we call perturbed penalized log-likelihood function, where $\boldsymbol{\omega}=\left(\omega_{1}, \cdots, \omega_{m}\right)^{\top}$ is a perturbation vector in some open subset $\Omega$ of $\mathbf{R}^{m}$ in which lives $\boldsymbol{\omega}_{0}$ a reference point in the analysis. At $\boldsymbol{\omega}_{0}$ the perturbed penalized log-likelihood function satisfies

$$
L_{p}\left(\boldsymbol{\theta} \mid \boldsymbol{\omega}_{0}, \boldsymbol{\alpha}\right)=L_{p}(\boldsymbol{\theta}, \boldsymbol{\alpha})
$$

So, we have the non-perturbed penalized log-likelihood function. Cook also employed the concept of the likelihood displacement function, extended here by

$$
f(\boldsymbol{\omega})=2\left[L_{p}\left(\hat{\boldsymbol{\theta}} \mid \boldsymbol{\omega}_{0}, \boldsymbol{\alpha}\right)-L_{p}\left(\hat{\boldsymbol{\theta}}_{\boldsymbol{\omega}} \mid \boldsymbol{\omega}_{\mathbf{0}}, \boldsymbol{\alpha}\right)\right]
$$


where $\hat{\boldsymbol{\theta}}$ and $\hat{\boldsymbol{\theta}}_{\boldsymbol{\omega}}$ are the maximum penalized likelihood estimates of $\boldsymbol{\theta}$ under $L_{p}\left(\boldsymbol{\theta} \mid \boldsymbol{\omega}_{0}, \boldsymbol{\alpha}\right)$ and $L_{p}(\boldsymbol{\theta} \mid \boldsymbol{\omega}, \alpha)$, respectively. Let

$$
\phi(\boldsymbol{\omega})=\left(\boldsymbol{\omega}^{\top}, f(\boldsymbol{\omega})\right)^{\top}
$$

be the influence graph. If the likelihood displacement function is a smooth function then the influence graph will be a regular surface and we can define normal curvatures. The essential idea, in the Cook's approach, is to study the normal curvatures for $\phi(\cdot)$ at $\left(\hat{\boldsymbol{\theta}}, \boldsymbol{\omega}_{0}\right)$ in the unitary direction $l$. Such curvature is expressed as

$$
C_{l}(\boldsymbol{\theta})=2\left|l^{\top} \Delta^{\top}\left(\mathbf{J}_{p}^{\hat{\theta} \hat{\theta}}\right)^{-1} \Delta l\right|,
$$

where $\mathbf{J}_{p}^{\hat{\theta} \hat{\theta}}$ denotes the observed information matrix of $\boldsymbol{\theta}$ evaluated at $\hat{\boldsymbol{\theta}}, \Delta=$ $\left(\Delta_{\beta \omega}^{\top}, \Delta_{\gamma_{1} \omega}^{p}, \cdots, \Delta_{\gamma_{k} \omega}^{\top}, \Delta_{\sigma \omega}^{\top}, \Delta_{\lambda \omega}^{\top}\right)^{\top}$ with $\Delta_{\beta \omega}$ and $\Delta_{\gamma_{l} \omega}$ being $(p \times n)$ and $\left(q_{l} \times\right.$ $n)$ matrices, respectively, with elements $\left(\Delta_{\beta \omega}\right)_{i j}=\frac{\partial^{2} L_{p}(\boldsymbol{\theta} \mid \omega, \alpha)}{\partial \beta_{j} \partial \omega_{i}}$ and $\left(\Delta_{\gamma_{l} \omega}\right)_{i j}=$ $\frac{\partial^{2} L_{p}(\boldsymbol{\theta} \mid \omega, \alpha)}{\partial \gamma_{j} l \partial \omega_{i}}$ for $i=1, \cdots, n, j=1, \cdots, q_{l}$, and $l=1, \cdots, k$, whereas $\Delta_{\sigma \omega}$ and $\Delta_{\lambda \omega}$ are $(1 \times n)$ vectors with elements $\left(\Delta_{\sigma \omega}\right)_{i}=\frac{\partial^{2} L_{p}(\boldsymbol{\theta} \mid \omega, \alpha)}{\partial \sigma \partial \omega_{i}}$ and $\left(\Delta_{\lambda \omega}\right)_{i}=$ $\frac{\partial^{2} L_{p}(\boldsymbol{\theta} \mid \omega, \alpha)}{\partial \lambda \partial \omega_{i}}$ for $i=1, \cdots, n$, respectively, evaluated at $\hat{\boldsymbol{\theta}}$ and $\boldsymbol{\omega}_{0}$.

Poon and Poon (1999) proposed the conformal normal curvature in order to have a curvature invariant under uniform change of scale, defined as

$$
B_{l}(\boldsymbol{\theta})=\frac{\left|l^{\top} \Delta^{\top}\left(\mathbf{J}_{p}^{\hat{\theta} \hat{\theta}}\right)^{-1} \Delta l\right|}{\sqrt{\operatorname{tr}\left(\Delta^{\top}\left(\mathbf{J}_{p}^{\hat{\theta} \hat{\theta}}\right)^{-1} \Delta\right)^{2}}} .
$$

This curvature has the property that for any unitary direction $l$ at $\hat{\boldsymbol{\theta}}$ we have that $0 \leq B_{l}(\boldsymbol{\theta}) \leq 1$. One way is to evaluate the normal curvature in the direction $l=e_{i}$, where $e_{i}$ is an $(n \times 1)$ vector with 1 at the $i$-th position and zeros in the remaining positions, and observing the index plot of $B_{e_{i}}$. According with Relvas and Paula (2016) we may use $B_{e_{i}}>\bar{B}+c^{*} S E(B)$ to discriminate if an observation is influential or not, where $\bar{B}$ is the mean of $B_{i}=B_{e_{i}}, i=1, \cdots, n$, $S E(B)$ denotes the standard error of $B_{i}$ and $c^{*}$ is selected appropriately.

\subsection{Perturbation schemes}

In this section we will derive the perturbation matrix $\Delta$ under some usual perturbation schemes applied to the models (3.1) and (4.1). For more details 
on the calculation see Appendix D.

\subsubsection{Case-weight perturbation: uncensored case}

For this perturbation scheme we assume that

$$
L_{p}(\boldsymbol{\theta}, \boldsymbol{\alpha} \mid \boldsymbol{\omega})=\sum_{i=1}^{n} \omega_{i} L_{i}(\boldsymbol{\theta})-\frac{\alpha_{1}}{2} \boldsymbol{\gamma}_{1}^{\top} \mathbf{M}_{1} \boldsymbol{\gamma}_{1}-\cdots-\frac{\alpha_{k}}{2} \boldsymbol{\gamma}_{k}^{\top} \mathbf{M}_{k} \boldsymbol{\gamma}_{k}
$$

where $L_{i}(\boldsymbol{\theta})$ denotes the $i$-th component of $L(\boldsymbol{\theta}), 0<\omega_{i} \leq 1$ and the reference point is $\boldsymbol{\omega}_{0}=(1, \cdots, 1)^{\top}$. Then, we obtain

$$
\begin{aligned}
\left(\Delta_{\beta \omega}\right)_{i j} & =-\frac{x_{i j}}{\lambda \sigma}+\frac{1}{\lambda \sigma} x_{i j} e^{\lambda \epsilon_{i}}, \quad j=1, \cdots, p \\
\left(\Delta_{\gamma_{l} \omega}\right)_{i j} & =-\frac{1}{\lambda \sigma} N_{i j l}+\frac{1}{\lambda \sigma} N_{i j l} e^{\lambda \epsilon_{i}}, \quad j=1, \cdots, q_{l} \\
\left(\Delta_{\sigma \omega}\right)_{i} & =-\frac{1}{\sigma}-\frac{\epsilon_{i}}{\lambda \sigma}+\frac{1}{\lambda \sigma} \epsilon_{i} e^{\lambda \epsilon_{i}} \text { and } \\
\left(\Delta_{\lambda \omega}\right)_{i} & =\zeta_{\lambda}-\frac{\epsilon_{i}}{\lambda^{2}}+\frac{2}{\lambda^{3}} e^{\lambda \epsilon_{i}}-\frac{1}{\lambda^{2}} \epsilon_{i} e^{\lambda \epsilon_{i}}
\end{aligned}
$$

for $i=1, \cdots, n, l=1, \cdots, k$, and $\zeta_{\lambda}=\frac{1}{\lambda}+\frac{2}{\lambda^{3}}\left\{\psi\left(\frac{1}{\lambda^{2}}\right)+2 \log |\lambda|-1\right\}$.

\subsubsection{Case-weight perturbation: censored case}

In this case we assume that

$L_{p}(\boldsymbol{\theta}, \boldsymbol{\alpha} \mid \boldsymbol{\omega})=\sum_{i} \omega_{i}\left[\left(1-\delta_{i}\right) L_{i}(\boldsymbol{\theta})+\delta_{i} \log \left(1-F_{\epsilon}\left(\epsilon_{i}, \boldsymbol{\theta}\right)\right)\right]-\frac{\alpha_{1}}{2} \boldsymbol{\gamma}_{1}^{\top} \mathbf{M}_{1} \boldsymbol{\gamma}_{1}-\cdots-\frac{\alpha_{k}}{2} \boldsymbol{\gamma}_{k}^{\top} \mathbf{M}_{k} \boldsymbol{\gamma}_{k}$

where $0<\omega_{i} \leq 1$, the reference point is $\boldsymbol{\omega}_{0}=(1, \cdots, 1)^{\top}$ and $F_{\epsilon}\left(\epsilon_{i} ; \boldsymbol{\theta}\right)$ is the cumulative distribution function of a generalized log-gamma distribution 
$\operatorname{GLG}(0,1, \lambda)$, more details see chapter 2 . If $\lambda>0$, we obtain

$$
\begin{aligned}
\left(\Delta_{\boldsymbol{\beta} \omega}\right)_{i j} & =\left(1-\delta_{i}\right)\left[-\frac{x_{i j}}{\lambda \sigma}+\frac{1}{\lambda \sigma} x_{i j} e^{\lambda \epsilon_{i}}\right]+\delta_{i}\left[x_{i j} \frac{\lambda}{\sigma \Gamma\left(\lambda^{-2}\right)} \frac{a_{i}^{\lambda^{-2}} e^{-a_{i}}}{1-\mathrm{I}\left(\lambda^{-2}, a_{i}\right)}\right] \\
\left(\Delta_{\gamma_{l} \omega}\right)_{i j} & =\left(1-\delta_{i}\right)\left[-\frac{1}{\lambda \sigma} N_{i j l}+\frac{1}{\lambda \sigma} N_{i j l} e^{\lambda \epsilon_{i}}\right]+\delta_{i}\left[N_{i j l} \frac{\lambda}{\sigma \Gamma\left(\lambda^{-2}\right)} \frac{a_{i}^{\lambda^{-2}} e^{-a_{i}}}{1-\mathrm{I}\left(\lambda^{-2}, a_{i}\right)}\right] \\
\left(\Delta_{\sigma \omega}\right)_{i} & =\left(1-\delta_{i}\right)\left[-\frac{1}{\sigma}-\frac{\epsilon_{i}}{\lambda \sigma}+\frac{1}{\lambda \sigma} \epsilon_{i} e^{\lambda \epsilon_{i}}\right]+\delta_{i}\left[\frac{\lambda}{\sigma \Gamma\left(\lambda^{-2}\right)} \frac{\epsilon_{i} a_{i}^{\lambda^{-2}} e^{-a_{i}}}{\left[1-\mathrm{I}\left(\lambda^{-2}, a_{i}\right)\right]}\right] \text { and } \\
\left(\Delta_{\lambda \omega}\right)_{i} & =\left(1-\delta_{i}\right)\left[\frac{1}{\lambda}+\frac{2}{\lambda^{3}} \psi\left(\frac{1}{\lambda^{2}}\right)-\frac{2}{\lambda^{3}}+4 \frac{\log (\lambda)}{\lambda^{3}}-\frac{\epsilon_{i}}{\lambda^{2}}+\frac{2}{\lambda^{3}} e^{\lambda \epsilon_{i}}-\frac{1}{\lambda^{2}} \epsilon_{i} e^{\lambda \epsilon_{i}}\right] \\
& +\delta_{i}\left[-\frac{2 \lambda^{-3} \psi\left(\lambda^{-2}\right) I\left(\lambda^{-2}, a_{i}\right)+\frac{1}{\Gamma\left(\lambda^{-2}\right)} a_{i}^{\lambda^{-2}} e^{-a_{i}}\left(\epsilon_{i}-2 \lambda^{-1}\right)}{1-\mathrm{I}\left(\lambda^{-2}, a_{i}\right)}\right]
\end{aligned}
$$

If $\lambda<0$, we obtain

$$
\begin{aligned}
\left(\Delta_{\beta \omega}\right)_{i j} & =\left(1-\delta_{i}\right)\left[-\frac{x_{i j}}{\lambda \sigma}+\frac{1}{\lambda \sigma} x_{i j} e^{\lambda \epsilon_{i}}\right]+\delta_{i}\left[-x_{i j} \frac{\lambda}{\sigma \Gamma\left(\lambda^{-2}\right)} \frac{a_{i}^{\lambda^{-2}} e^{-a_{i}}}{\mathrm{I}\left(\lambda^{-2}, a_{i}\right)}\right] \\
\left(\Delta_{\gamma_{l} \omega}\right)_{i j} & =\left(1-\delta_{i}\right)\left[-\frac{1}{\lambda \sigma} N_{i j l}+\frac{1}{\lambda \sigma} N_{i j l} e^{\lambda \epsilon_{i}}\right] \delta_{i}\left[-N_{i j l} \frac{\lambda}{\sigma \Gamma\left(\lambda^{-2}\right)} \frac{a_{i}^{\lambda^{-2}} e^{-a_{i}}}{1-\mathrm{I}\left(\lambda^{-2}, a_{i}\right)}\right] \\
\left(\Delta_{\sigma \omega}\right)_{i} & =\left(1-\delta_{i}\right)\left[-\frac{1}{\sigma}-\frac{\epsilon_{i}}{\lambda \sigma}+\frac{1}{\lambda \sigma} \epsilon_{i} e^{\lambda \epsilon_{i}}\right]+\delta_{i}\left[-\frac{\lambda}{\sigma \Gamma\left(\lambda^{-2}\right)} \frac{\epsilon_{i} a_{i}^{\lambda^{-2}} e^{-a_{i}}}{\left[\mathrm{I}\left(\lambda^{-2}, a_{i}\right)\right]}\right] \text { and } \\
\left(\Delta_{\lambda \omega}\right)_{i} & =\left(1-\delta_{i}\right)\left[\frac{1}{\lambda}+\frac{2}{\lambda^{3}} \psi\left(\frac{1}{\lambda^{2}}\right)-\frac{2}{\lambda^{3}}+4 \frac{\log (\lambda)}{\lambda^{3}}-\frac{\epsilon_{i}}{\lambda^{2}}+\frac{2}{\lambda^{3}} e^{\lambda \epsilon_{i}}-\frac{1}{\lambda^{2}} \epsilon_{i} e^{\lambda \epsilon_{i}}\right] \\
& +\delta_{i}\left[\frac{2 \lambda^{-3} \psi\left(\lambda^{-2}\right) I\left(\lambda^{-2}, a_{i}\right)+\frac{1}{\Gamma\left(\lambda^{-2}\right)} a_{i}^{\lambda^{-2}} e^{-a_{i}}\left(\epsilon_{i}-2 \lambda^{-1}\right)}{\mathrm{I}\left(\lambda^{-2}, a_{i}\right)}\right]
\end{aligned}
$$

where $a_{i}=\lambda^{-2} e^{\lambda \epsilon_{i}}$ and $i=1, \cdots, n ; l=1, \cdots, k$.

\subsubsection{Response perturbation: uncensored case}

For this perturbation scheme we consider that each $y_{i}$ is perturbed as

$$
y_{i}^{\prime}=y_{i}+\omega_{i}
$$

Hence,

$$
\epsilon_{i \boldsymbol{\omega}}=\frac{y_{i}^{\prime}-\mathbf{x}_{i}^{\top} \boldsymbol{\beta}-\mathbf{N}_{i 1} \boldsymbol{\gamma}_{1}-\cdots-\mathbf{N}_{i k} \boldsymbol{\gamma}_{k}}{\sigma}
$$


$\omega_{i} \in \mathbf{R}$ and $\boldsymbol{\omega}_{0}=(0, \cdots, 0)^{\top}$. So, we obtain

$$
\begin{aligned}
\left(\Delta_{\boldsymbol{\beta} \omega}\right)_{i j} & =\frac{1}{\sigma^{2}} x_{i j} e^{\lambda \epsilon_{i \omega}}, \quad j=1, \cdots, p, \\
\left(\Delta_{\gamma_{l} \omega}\right)_{i j} & =\frac{1}{\sigma^{2}} N_{i j l} e^{\lambda \epsilon_{i \omega}}, \quad j=1, \cdots, q_{l}, \\
\left(\Delta_{\sigma \omega}\right)_{i} & =\frac{1}{\lambda \sigma^{2}}\left[\lambda \epsilon_{i_{\omega}} e^{\lambda \epsilon_{i_{\omega}}}+e^{\lambda \epsilon_{i_{\omega}}}-1\right] \text { and } \\
\left(\Delta_{\lambda \omega}\right)_{i} & =-\frac{1}{\lambda^{2} \sigma}+\frac{1}{\lambda^{2} \sigma} e^{\lambda \epsilon_{i_{\omega}}}-\frac{1}{\lambda \sigma} \epsilon_{i_{\omega}} e^{\lambda \epsilon_{i_{\omega}}},
\end{aligned}
$$

for $i=1, \cdots, n$ and $l=1, \cdots, k$.

\subsubsection{Response perturbation: censored case}

If $\lambda>0$, we obtain

$$
\begin{aligned}
\left(\Delta_{\boldsymbol{\beta} \omega}\right)_{i j} & =\left(1-\delta_{i}\right)\left[\frac{1}{\sigma^{2}} x_{i j} e^{\lambda \epsilon_{i \omega}}\right]+\delta_{i}\left[\frac{-\lambda^{2}}{\sigma^{2} \Gamma\left(\lambda^{-2}\right)} \frac{x_{i j} a_{i}^{\lambda^{-2}} e^{-a_{i}}}{1-\mathrm{I}\left(\lambda^{-2}, a_{i}\right)}\left(a_{i}-\frac{1}{\lambda^{2}}-\frac{a_{i}^{\lambda^{-2}} e^{-a_{i}}}{1-\mathrm{I}\left(\lambda^{-2}, a_{i}\right)}\right)\right] \\
\left(\Delta_{\gamma_{l} \omega}\right)_{i j} & =\left(1-\delta_{i}\right)\left[\frac{1}{\sigma^{2}} N_{i j l} e^{\lambda \epsilon_{i \omega}}\right]+\delta_{i}\left[\frac{-\lambda^{2}}{\sigma^{2} \Gamma\left(\lambda^{-2}\right)} \frac{N_{i j l} a_{i}^{\lambda^{-2}} e^{-a_{i}}}{1-\mathrm{I}\left(\lambda^{-2}, a_{i}\right)}\left(a_{i}-\frac{1}{\lambda^{2}}-\frac{a_{i}^{\lambda^{-2}} e^{-a_{i}}}{1-\mathrm{I}\left(\lambda^{-2}, a_{i}\right)}\right)\right] \\
\left(\Delta_{\sigma \omega}\right)_{i} & =\left(1-\delta_{i}\right)\left[\frac{1}{\lambda \sigma^{2}}\left(\lambda \epsilon_{i_{\omega}} e^{\lambda \epsilon_{i \omega}}+e^{\lambda \epsilon_{i \omega}}-1\right)\right] \\
& +\delta_{i}\left[\frac{\lambda^{2}}{\sigma^{2} \Gamma\left(\lambda^{-2}\right)} \frac{a_{i}^{\lambda^{-2}} e^{-a_{i}}}{1-\mathrm{I}\left(\lambda^{-2}, a_{i}\right)}\left[\epsilon_{i}\left(a_{i}-\frac{1}{\lambda^{2}}-\frac{a_{i}^{\lambda^{-2}} e^{-a_{i}}}{1-\mathrm{I}\left(\lambda^{-2}, a_{i}\right)}\right)-\frac{1}{\lambda}\right]\right] \text { and } \\
\left(\Delta_{\lambda \omega}\right)_{i} & =\left(1-\delta_{i}\right)\left[-\frac{1}{\lambda^{2} \sigma}+\frac{1}{\lambda^{2} \sigma} e^{\lambda \epsilon_{i \omega}}-\frac{1}{\lambda \sigma} \epsilon_{i_{\omega}} e^{\lambda \epsilon_{i \omega}}\right] \\
& +\delta_{i}\left[\frac { x _ { i j } a _ { i } ^ { \lambda ^ { - 2 } } e ^ { - a _ { i } } } { \sigma \Gamma ( \lambda ^ { - 2 } ) S ( a _ { i } , \lambda ) } \left[1+\lambda^{-1}\left(\epsilon_{i}-2 \lambda^{-1}\left(\log a_{i}+1\right)\right)+\lambda a_{i}\left(2 \lambda^{-1}-\epsilon_{i}\right)+2 \lambda^{-2} \psi\left(\lambda^{-2}\right)\right.\right. \\
& \left.\left.+\frac{\lambda}{S\left(a_{i}, \lambda\right)}\left(2 \lambda^{-3} \psi\left(\lambda^{-2}\right) I\left(\lambda^{-2}, a_{i}\right)+\frac{1}{\Gamma\left(\lambda^{-2}\right)} a_{i}^{\lambda^{-2}} e^{-a_{i}}\left(\epsilon_{i}-2 \lambda^{-1}\right)\right)\right]\right]
\end{aligned}
$$


And, if $\lambda<0$, we obtain

$$
\begin{aligned}
\left(\Delta_{\boldsymbol{\beta} \omega}\right)_{i j} & =\left(1-\delta_{i}\right)\left[\frac{1}{\sigma^{2}} x_{i j} e^{\lambda \epsilon_{i \omega}}\right]+\delta_{i}\left[\frac{-\lambda^{2}}{\sigma^{2} \Gamma\left(\lambda^{-2}\right)} \frac{x_{i j} a_{i}^{\lambda^{-2}} e^{-a_{i}}}{1-\mathrm{I}\left(\lambda^{-2}, a_{i}\right)}\left(-a_{i}+\frac{1}{\lambda^{2}}-\frac{a_{i}^{\lambda^{-2}} e^{-a_{i}}}{1-\mathrm{I}\left(\lambda^{-2}, a_{i}\right)}\right)\right], \\
\left(\Delta_{\gamma_{l} \omega}\right)_{i j} & =\left(1-\delta_{i}\right)\left[\frac{1}{\sigma^{2}} N_{i j l} e^{\lambda \epsilon_{i \omega}}\right]+\delta_{i}\left[\frac{-\lambda^{2}}{\sigma^{2} \Gamma\left(\lambda^{-2}\right)} \frac{N_{i j l} a_{i}^{\lambda^{-2}} e^{-a_{i}}}{1-\mathrm{I}\left(\lambda^{-2}, a_{i}\right)}\left(-a_{i}+\frac{1}{\lambda^{2}}-\frac{a_{i}^{\lambda^{-2}} e^{-a_{i}}}{1-\mathrm{I}\left(\lambda^{-2}, a_{i}\right)}\right)\right], \\
\left(\Delta_{\sigma \omega}\right)_{i} & =\left(1-\delta_{i}\right)\left[\frac{1}{\lambda \sigma^{2}}\left(\lambda \epsilon_{i_{\omega}} e^{\lambda \epsilon_{i_{\omega}}}+e^{\lambda \epsilon_{i_{\omega}}}-1\right)\right] \\
& +\delta_{i}\left[\frac{\lambda^{2}}{\sigma^{2} \Gamma\left(\lambda^{-2}\right)} \frac{a_{i}^{\lambda^{-2}} e^{-a_{i}}}{1-\mathrm{I}\left(\lambda^{-2}, a_{i}\right)}\left[\epsilon_{i}\left(a_{i}-\frac{1}{\lambda^{2}}-\frac{a_{i}^{\lambda^{-2}} e^{-a_{i}}}{1-\mathrm{I}\left(\lambda^{-2}, a_{i}\right)}\right)-\frac{1}{\lambda}\right]\right] \text { and } \\
\left(\Delta_{\lambda \omega}\right)_{i} & =\left(1-\delta_{i}\right)\left[-\frac{1}{\lambda^{2} \sigma}+\frac{1}{\lambda^{2} \sigma} e^{\lambda \epsilon_{i \omega}}-\frac{1}{\lambda \sigma} \epsilon_{i_{\omega}} e^{\lambda \epsilon_{i \omega}}\right] \\
& +\delta_{i}\left[\frac { x _ { i j } a _ { i } ^ { \lambda ^ { - 2 } } e ^ { - a _ { i } } } { \sigma \Gamma ( \lambda ^ { - 2 } ) S ( a _ { i } , \lambda ) } \left[1+\lambda^{-1}\left(\epsilon_{i}-2 \lambda^{-1}\left(\log a_{i}+1\right)\right)+\lambda a_{i}\left(2 \lambda^{-1}-\epsilon_{i}\right)+2 \lambda^{-2} \psi\left(\lambda^{-2}\right)\right.\right. \\
& \left.\left.+\frac{\lambda}{S\left(a_{i}, \lambda\right)}\left\{2 \lambda^{-3} \psi\left(\lambda^{-2}\right) I\left(\lambda^{-2}, a_{i}\right)+\frac{1}{\Gamma\left(\lambda^{-2}\right)} a_{i}^{\lambda^{-2}} e^{-a_{i}}\left(\epsilon_{i}-2 \lambda^{-1}\right)\right\}\right]\right]
\end{aligned}
$$

for $i=1, \cdots, n$ and $l=1, \cdots, k$.

Several diagnostic graphs may be performed with the quantities derived in the previous sections. For instance, graph between each proposed residual against the fitted value and normal probability plots with simulated confidence bands to detect outlying observations, departures from model assumptions, such as error assumption. In addition, index plot of $B_{i}(\boldsymbol{\theta})$ may be performed to assess the sensitivity of the maximum penalized likelihood estimates $\hat{\boldsymbol{\theta}}$ under the perturbation schemes. Similarly, one may perform index plots of $B_{i}(\boldsymbol{\beta})$, $B_{i}(\sigma)$ and $B_{i}(\lambda)$ (see, for instance, Relvas and Paula, 2016). 


\section{Chapter 6}

\section{The sglg package}

\subsection{Introduction}

In the $\mathbf{R}$ computational ambient there is only one package robustloggamma, Agostinelli et al. (2017), that allows to fit regression models with generalized log-gamma errors. Nevertheless, if we want to specify a non-parametric effect we can not do it. Additionally, there exist no any checking or sensitivity analysis tool in this package. Therefore, the new $\mathbf{R}$ package sglg, Cardozo et al. (2017), is considered. The objective of this chapter is to describe the package sglg, which allows to fit semi-parametric generalized log-gamma regression models considering the possibility of censored observations. We illustrate below some of its functions, how to use, its arguments, its values and some examples.

\subsubsection{Function glg}

glg Fitting multiple linear generalized log-gamma regression models

\section{Description}

glg may be used to fit a multiple linear regression model suitable for analysis of data sets in which the response variable is continuous, strictly positive and asymmetric. However, glg may also be used to fit data for which the response is asymmetric in the real numbers. Under this setup, the location parameter of the response variable distribution is explicitly modeled by a linear model of the parameters. 
Usage

glg(formula, data, Tolerance, Maxiter)

\section{Arguments}

formula a symbolic description of the systematic component of the model to be fitted.

data an optional data frame, list or environment containing the variables in the model.

Tolerance an optional positive value, which represents the convergence criterion. Default value is $1 \mathrm{e}-04$.

Maxiter an optional positive integer giving the maximal number of iterations for the estimating process. Default value is $1 \mathrm{e} 03$.

Value

$\mathrm{mu} \quad$ a vector of parameter estimates associated with the model.

sigma estimate of the scale parameter associated with the model.

lambda estimate of the shape parameter associated with the model.

interval estimate of a 95\% confidence interval for each estimate parameter associated with the model.

$\mathrm{p}$.value gives the p-value associated with each estimate parameter associated with the model.

Deviance the deviance associated with the model.

\section{Example}

\# Multiple linear generalized log-gamma regression model

set.seed (8142031)

rows $<-200$

columns $<-2$

$\mathrm{X}<-$ matrix (0, rows, columns)

$X[, 1]<-r b i n o m$ (rows $, 1,0.5$ )

$\mathrm{X}[, 2]<-r u n i f$ (rows , 0,1 ) 


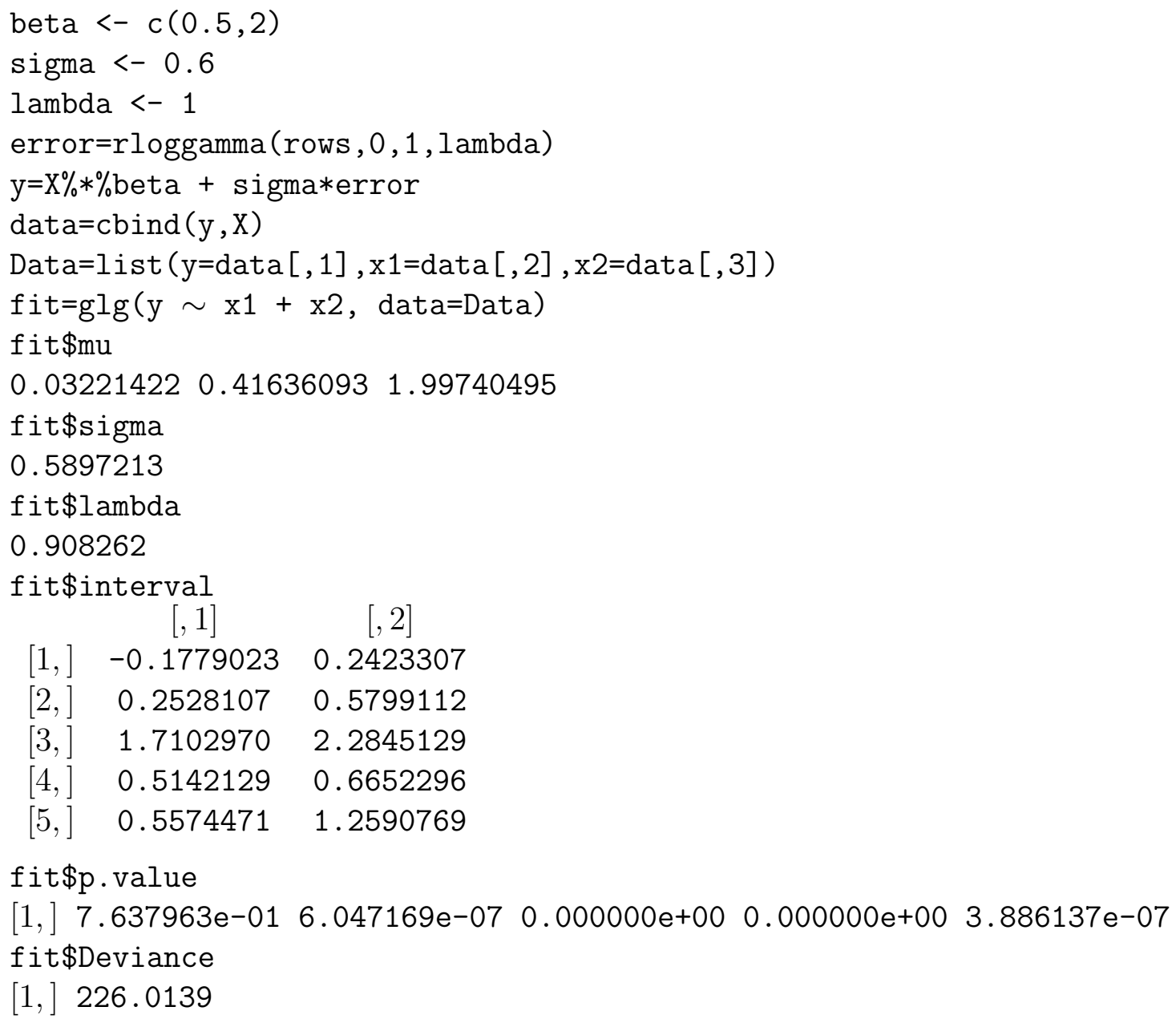

fit $\$ p$.value

[1,] $7.637963 e-01 \quad 6.047169 e-07 \quad 0.000000 e+00 \quad 0.000000 e+00 \quad 3.886137 e-07$ fit\$Deviance

$[1]$,

The item fit\$interval describes the interval estimates which appear for $\beta_{0}, \beta_{1}, \beta_{2}$, scale and shape parameters, respectively.

\subsubsection{Function sglg}

sglg Fitting semi-parametric generalized log-gamma regression models

\section{Description}

sglg may be used to fit a semi-parametric regression model suitable for analysis of data sets in which the response variable is continuous, strictly positive and 
asymmetric. However, sglg may also be used to fit data for which the response is asymmetric in the real numbers. Under this setup, the location parameter of the response variable distribution is explicitly modeled by semi-parametric functions, whose non-parametric components may be approximated by natural cubic splines.

\section{Usage}

sglg(formula, npc, basis, data, shape, method, Tolerance, Maxiter)

\section{Arguments}

formula a symbolic description of the linear components of the systematic part of the model to be fitted.

npc a data frame with potential non-parametric variables of the systematic part of the model to be fitted.

basis a name of the cubic spline basis to be used in the model. Supported basis include De Boor and Gu basis which are a B-spline basis and a natural cubic spline basis, respectively.

data an optional data frame, list or environment containing the variables in the model.

shape an optional value for the shape parameter of the error distribution of a generalized log-gamma distribution. Default value is 1 .

method There are two possibles algorithms to estimate the parameters. The default algorithm is 'FS' Fisher-Scoring, the other option is 'GSFS' an adequate combination between the block matrix version of non-linear GaussSeidel algorithm and Fisher-Scoring algorithm.

Tolerance an optional positive value, which represents the convergence criterion. Default value is $1 \mathrm{e}-04$.

Maxiter an optional positive integer giving the maximal number of iterations for the estimating process. Default value is $1 \mathrm{e}-03$.

\section{Value}


$\mathrm{mu}$ a vector of parameter estimates associated with the systematic part of the model.

sigma estimate of the scale parameter associated with the model.

lambda estimate of the shape parameter associated with the model.

interval estimate of a $95 \%$ confidence interval for each estimate parameter associated with the model.

Deviance the deviance associated with the model.

\section{Example}

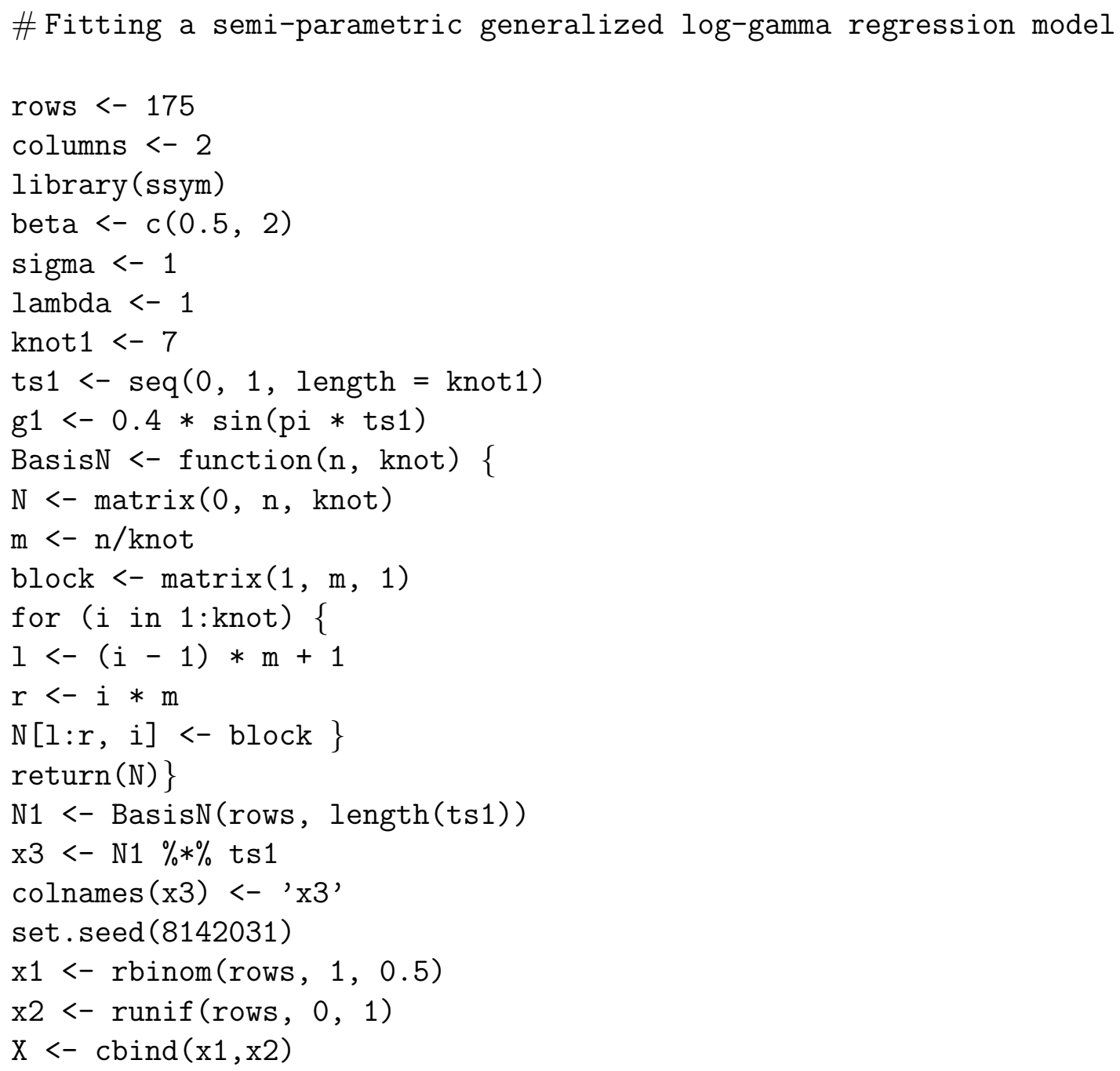




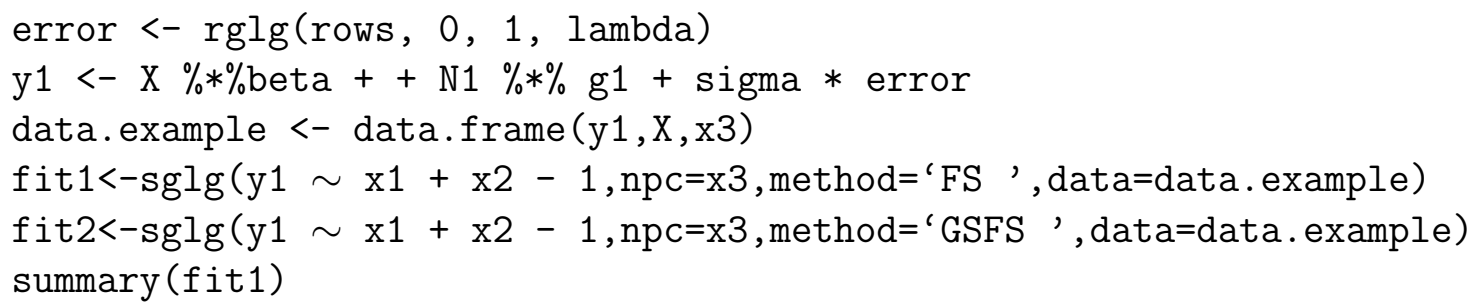

AIC : 571.83

BIC: 598.96 


\subsubsection{Function survglg}

survglg Fitting linear generalized log-gamma regression models under the presence of right censored data

\section{Description}

survglg may be used to fit a multiple linear regression model in which the response variable is continuous, strictly positive, asymmetric and there are right censored observations. In this setup, the location parameter of the logarithm of the response variable is modeled by a linear model of the parameters.

\section{Usage}

survglg(formula, data, Tolerance, Maxiter)

\section{Arguments}

formula a symbolic description of the systematic component of the model to be fitted.

data an optional data frame, list or environment containing the variables in the model.

Tolerance an optional positive value, which represents the convergence criterion. Default value is $1 \mathrm{e}-04$.

Maxiter an optional positive integer giving the maximal number of iterations for the estimating process. Default value is $1 \mathrm{e} 03$.

\section{Value}


$\mathrm{mu} \quad$ a vector of parameter estimates associated with the model.

sigma estimate of the scale parameter associated with the model.

lambda estimate of the shape parameter associated with the model.

per.cens percentage of censored observations in the data.

interval estimate of a $95 \%$ confidence interval for each estimate parameter associated with the model.

Deviance the deviance associated with the model.

\section{Example}

\# Multiple linear generalized log-gamma regression model under the presence of right censored data.

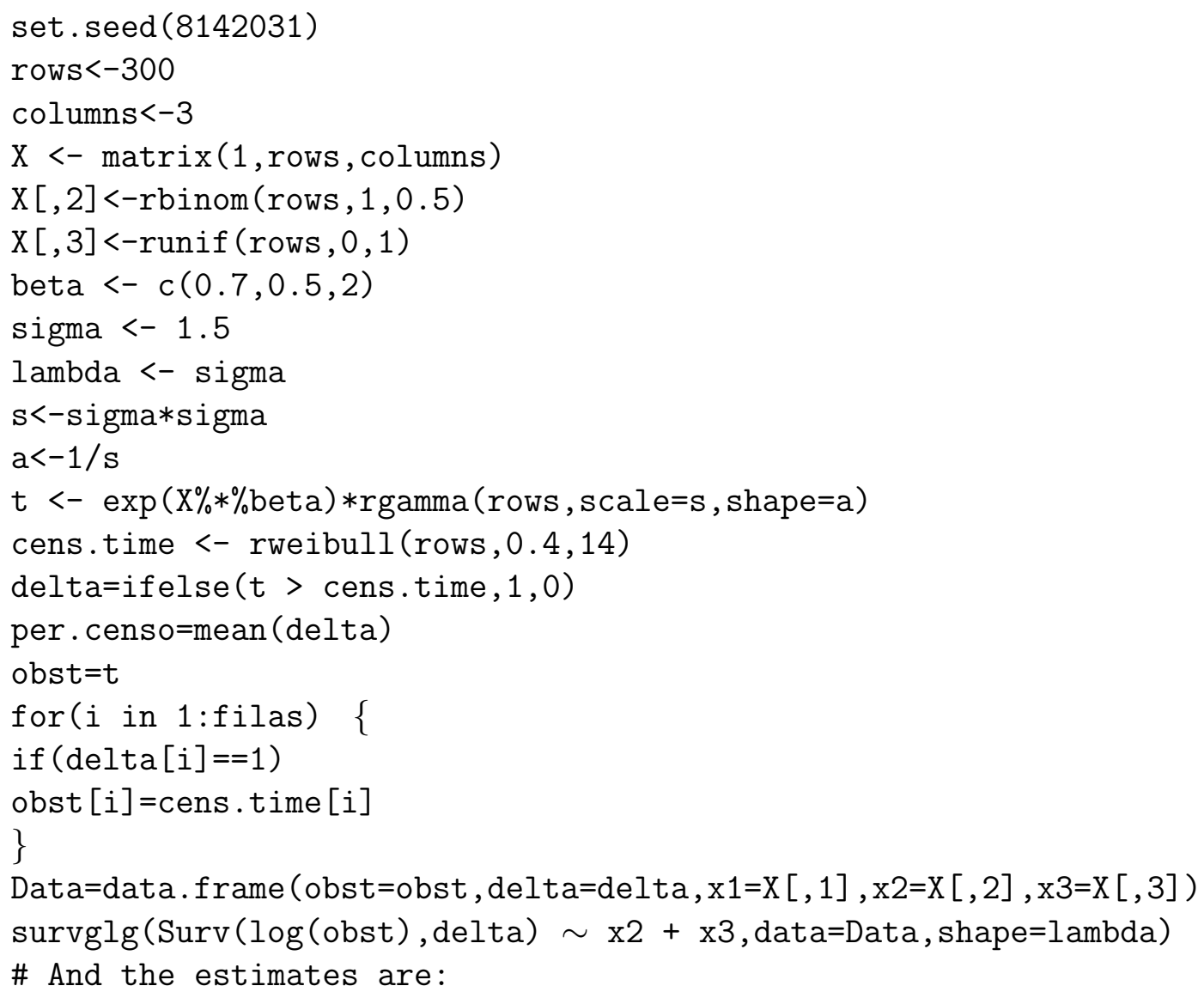


fit\$per.cens

37.5

fit $\$ \mathrm{mu}$

$\begin{array}{llll}0.5878320 & 0.7924222 & 1.9773314\end{array}$

fit $\$$ sigma

1.347329

fit\$interval

0.10116091 .074503

0.33472091 .250124

1.19737392 .757289

$1.1750197 \quad 1.519638$

The item fit\$interval describes the interval estimates which appear for $\beta_{0}, \beta_{1}, \beta_{2}$, and scale parameters, respectively.

\subsubsection{Function ssurvglg}

ssurvglg Fitting semi-parametric generalized log-gamma regression models under the presence of right censored data

\section{Description}

ssurvglg may be used to fit a semi-parametric regression model suitable for analysis of data sets in which the response variable is continuous, strictly positive and asymmetric. Under this setup, the location parameter of the logarithm of the response variable is explicitly modeled through semi-parametric functions, whose non-parametric components may be approximated by natural cubic splines.

\section{Usage}

ssurvglg (formula, npc, basis, data, shape, maxiter, tolerance)

\section{Arguments}


formula a symbolic description of the systematic component of the model to be fitted.

npc a data frame with potential nonparametric variables of the systematic part of the model to be fitted.

basis a name of the cubic spline basis to be used in the model. Supported basis include deBoor and $\mathrm{Gu}$ basis which are a B-spline basis and a natural cubic spline basis, respectively.

data an optional data frame, list or environment containing the variables in the model.

shape an optional value for the shape parameter of the model.

Tolerance an optional positive value, which represents the convergence criterion. Default value is $1 \mathrm{e}-04$.

Maxiter an optional positive integer giving the maximal number of iterations for the estimating process. Default value is $1 \mathrm{e} 03$.

\section{Value}

$\mathrm{mu}$

a vector of parameter estimates associated with the model.

sigma estimate of the scale parameter associated with the model.

lambda estimate of the shape parameter associated with the model.

Deviance the deviance associated with the model.

\section{Example}

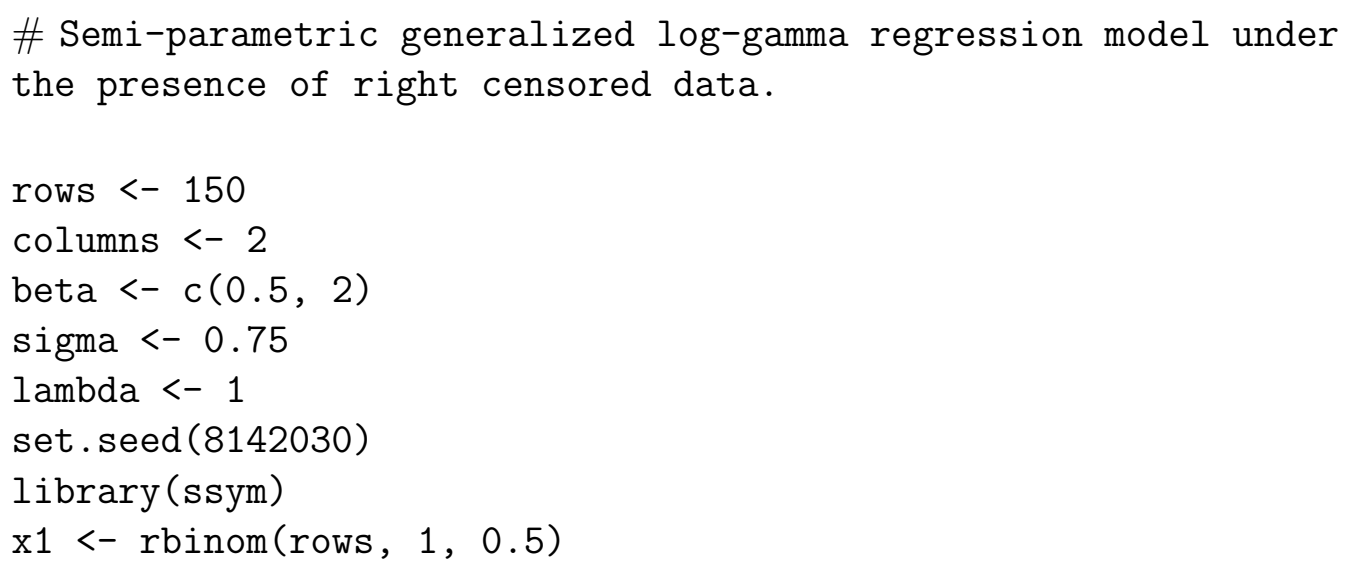




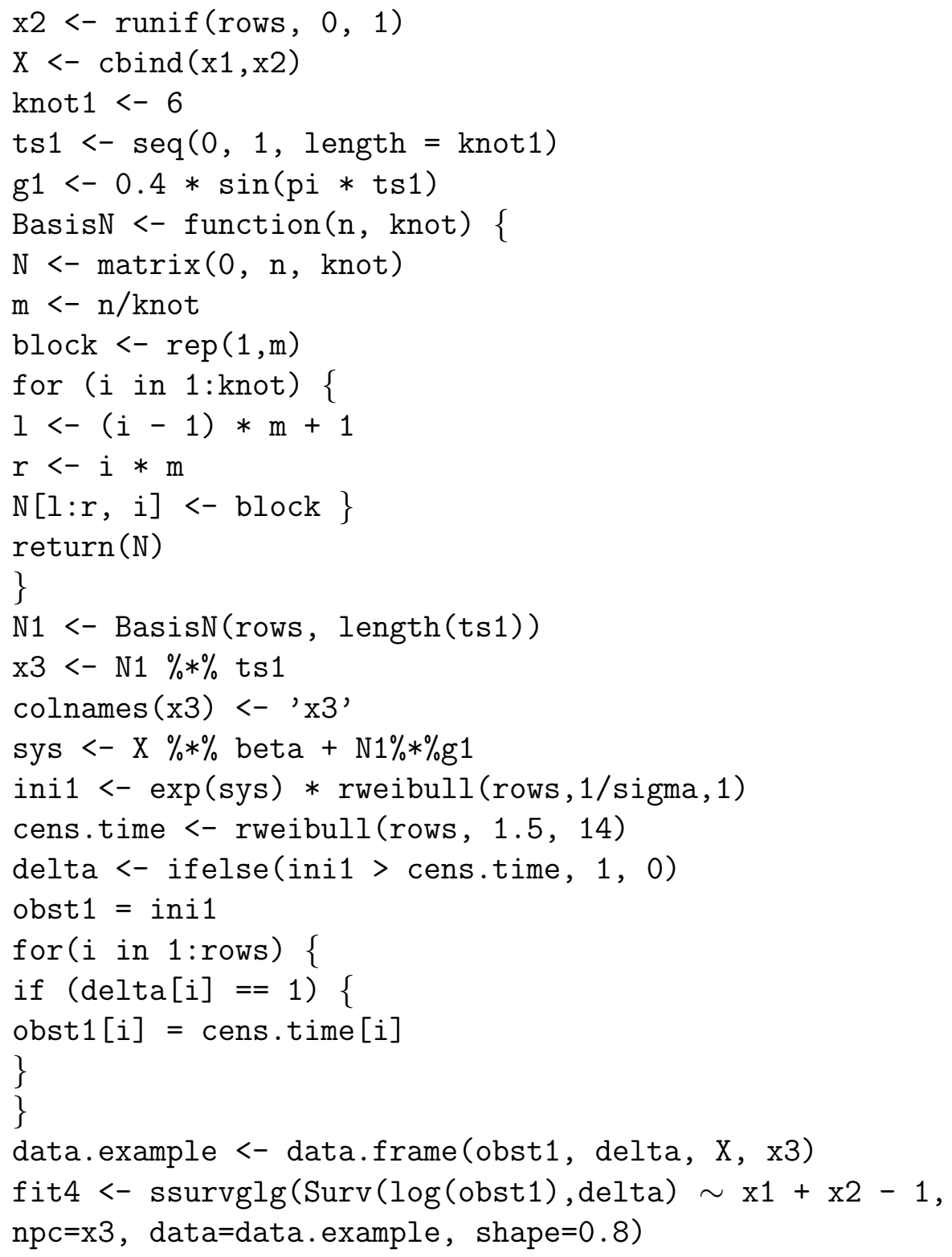




\title{
6.1.5 Function shape
}

\author{
shape Tool that supports the estimation of the shape parameter in semi- \\ parametric generalized log-gamma regression model under the pres- \\ ence of censored data
}

\section{Description}

shape may be used for the estimation of the shape parameter in semi-parametric or multiple linear accelerated failure time model with generalized log-gamma errors under the presence of censored data. The estimation is based on the profiled likelihood function for the shape parameter of the model.

\section{Usage}

shape (formula, npc, data, interval, semi, step)

\section{Arguments}

formula a symbolic description of the systematic component of the model to be fitted.

npc a data frame with potential non-parametric variables of the systematic part of the model to be fitted.

data an optional data frame, list or environment containing the variables in the model.

interval an optional numerical vector of length 2 . In this interval is the maximum likelihood estimate of the shape parameter of the model. By default is $[0.1,1.5]$.

semi a logical value. TRUE means that the model has a nonparametric component.

step an optional positive value. This parameter represents the length of the step of the partition of the interval parameter. By default is 0.1 .

\section{Example}

rows $<-200$

columns $<-2$ 


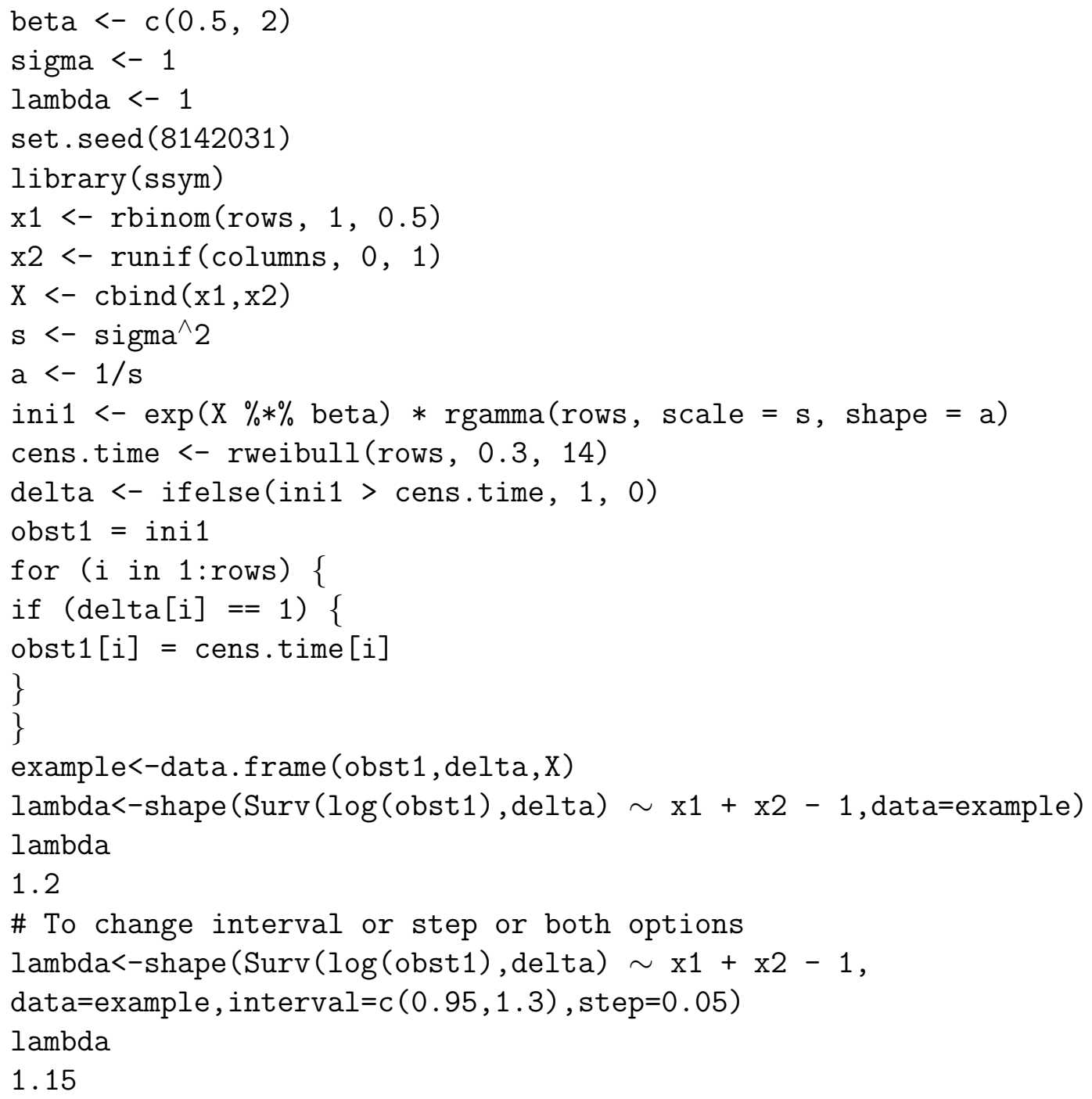

\subsubsection{Function AIC.sglg}

AIC.sglg AIC.sglg

\section{Description}


AIC.sglg calculates the goodness-of-fit-statistic AIC from an object of class "sglg".

\subsubsection{Function SIC.sglg}

SIC.sglg SIC.sglg

\section{Description}

SIC.sglg calculates the goodness-of-fit-statistic SIC from an object of class "sglg".

\subsubsection{Function envelope.sglg}

envelope Building normal probability plot with simulated envelope for the deviance-type residual

\section{Description}

envelope is used to compute and displaying normal probability plot with simulated envelope for the deviance-type residual

\section{Usage}

envelope.sglg(fit, Rep)

\section{Arguments}

fit an object of the class sglg. This object is returned from the call to $\operatorname{glg}(), \operatorname{sglg}()$.

Rep a positive integer. This is the number of replications on which to build the simulated envelope. Default is $\operatorname{Rep}=50$. 


\subsubsection{Function rglg}

rglg Random number generation for a generalized log-gamma
distribution

\section{Description}

rglg is used to generate random numbers from a generalized log-gamma distribution

\section{Usage}

rglg(n, location, scale, shape)

\section{Arguments}

$\mathrm{n} \quad$ numeric, size of the random sample.

location numeric, represent the location parameter. Default value is 0 .

scale numeric, represent the scale parameter. Default value is 1.

shape numeric, represent the shape parameter. Default value is 1 .

\section{Examples}

$\operatorname{rglg}(10$, location $=-1$, scale $=0.5$, shape $=1)$

\subsubsection{Some other functions}

Another functions that we may use in the package sglg are:

- Function lss is used to obtain the mean, variance, skewness and kurtosis for a generalized log-gamma distribution.

- Function sglg with penalized B-spline approach.

- Function ssurvglg with penalized B-spline approach. 
- Graph of the overall-goodness-of-fit for generalized log-gamma regression model fitted by glg.

- Graph of local influence for generalized log-gamma regression model fitted by glg under case-weight and response perturbation schemes.

- Graph of the non-parametric components with simultaneous confidence intervals for semi-parametric generalized log-gamma regression model fitted by sglg.

- Graph of the overall-goodness-of-fit for semi-parametric generalized loggamma regression model fitted by sglg.

- Graph of local influence for semi-parametric generalized log-gamma regression model fitted by sglg under case-weight and response perturbation schemes.

- Graph of the survival function for generalized log-gamma regression model fitted by survglg.

- Graph of the overall-goodness-of-fit for generalized log-gamma regression model fitted by survglg.

- Graph of local influence for generalized log-gamma regression model fitted by survglg.

- Graph of the survival function for semi-parametric generalized log-gamma regression model fitted by ssurvglg.

- Graph of the overall-goodness-of-fit for semi-parametric generalized loggamma regression model fitted by ssurvglg.

- Graph of local of influence for semi-parametric generalized log-gamma regression model fitted by ssurvglg.

More details about the r package sglg, see, Cardozo et al. (2017). 


\section{Chapter 7}

\section{Simulations and applications}

The aim of this chapter is to present some simulation studies and two applications of the methodologies developed in the previous chapters. We study, in the first section, some statistical properties of the parameter estimates from generalized log-gamma regression models through Monte Carlo simulations. In the second section, we analyze a real data set under semi-parametric generalized log-gamma regression models without censoring. Finally, in the last section, we analyze a real data set through semi-parametric generalized log-gamma regression models under the presence of right censoring.

\subsection{Simulation studies}

\subsubsection{Case: glg}

The function glg allows to fit a generalized log-gamma linear regression model, with a complete estimation of the parameters, location, scale and shape. There is a routine in the $\mathrm{R}$ package named robustloggamma that allows to fit generalized log-gamma linear regression models, but it does not provide any diagnostic

tool. For other hand, we offer some diagnostic functions, such as, envelope function for deviance residuals and local influence functions under case-weight and response variable perturbation schemes.

Data sets of size $n=100,200,300$ and 500 were generated from the model

$$
y_{i}=\beta_{0}+\beta_{1} x_{i 1}+\beta_{2} x_{i 2}+\sigma \epsilon_{i}, \text { for } i=1, \cdots, n,
$$

where $x_{1} \sim \operatorname{Bernoulli}(0.5), x_{2} \sim \operatorname{Unif}(0,1), \sigma>0$ and $\epsilon \sim \operatorname{GLG}(0,1, \lambda)$. 
The values of the parameters were $\beta_{0}=1, \beta_{1}=0.5, \beta_{2}=2, \sigma=0.6, \lambda=$ $-1,0.7,1.35$. The number of replicates was $\mathrm{R}=5000$, keeping the values of $x_{1}$ and $x_{2}$ fixed. The summary measures considered are given below.

Mean of the estimates

$$
\hat{\theta}=\frac{1}{R} \sum_{j=1}^{R} \hat{\theta}^{j}
$$

where $\hat{\theta}^{j}$ denotes the estimated value of the parameter in the $j$-th replication. Coverage rate for $\theta$ based on a $95 \%$ confidence interval

$$
C R(\hat{\theta})=\frac{100}{R} \sum_{j=1}^{R} I\left[\hat{\theta}^{j}-1.96\left[\widehat{\operatorname{Var}}\left(\hat{\theta}^{j}\right)\right]^{\frac{1}{2}}<\theta<\hat{\theta}^{j}+1.96\left[\widehat{\operatorname{Var}}\left(\hat{\theta}^{j}\right)\right]^{\frac{1}{2}}\right] .
$$

Tables 7.1.1 - 7.1.3 summarize the results of the simulations. From the results described in Tables 7.1.1 - 7.1.3, we may notice indication of consistence of the maximum likelihood estimates as the sample size increases. Similarly, the coverage rate for empirical $95 \%$ confidence intervals of each parameter becomes close to the nominal level as the sample size increases.

Table 7.1.1. Average of the parameter estimates and average of the coverage rates from the simulation study under $\lambda=-1$.

\begin{tabular}{c|ccccc|ccccc}
\hline$\lambda=-1$ & \multicolumn{5}{|c|}{$\theta$} & \multicolumn{5}{c}{$C R(\theta)$} \\
\hline$n$ & $\beta_{0}$ & $\beta_{1}$ & $\beta_{2}$ & $\sigma$ & $\lambda$ & $\beta_{0}$ & $\beta_{1}$ & $\beta_{2}$ & $\sigma$ & $\lambda$ \\
\hline 100 & 0.99 & 0.50 & 2.00 & 0.58 & -1.16 & 90.86 & 91.32 & 93.48 & 87.57 & 88.80 \\
200 & 1.00 & 0.50 & 1.99 & 0.59 & -1.00 & 94.11 & 92.72 & 94.47 & 90.68 & 91.34 \\
300 & 1.00 & 0.49 & 2.00 & 0.59 & -1.00 & 93.86 & 94.52 & 93.86 & 92.25 & 92.78 \\
\hline
\end{tabular}

Table 7.1.2. Average of the parameter estimates and average of the coverage rates from the simulation study under $\lambda=0.7$.

\begin{tabular}{c|ccccc|ccccc}
\hline$\lambda=0.7$ & \multicolumn{5}{|c|}{$\theta$} & \multicolumn{5}{c}{$C R(\theta)$} \\
\hline$n$ & $\beta_{0}$ & $\beta_{1}$ & $\beta_{2}$ & $\sigma$ & $\lambda$ & $\beta_{0}$ & $\beta_{1}$ & $\beta_{2}$ & $\sigma$ & $\lambda$ \\
\hline 100 & 1.00 & 0.50 & 2.00 & 0.57 & 0.72 & 92.36 & 93.24 & 93.12 & 88.68 & 91.44 \\
200 & 0.99 & 0.50 & 2.00 & 0.59 & 0.68 & 93.82 & 94.46 & 93.78 & 91.10 & 92.66 \\
300 & 1.00 & 0.49 & 1.99 & 0.59 & 0.69 & 94.50 & 94.54 & 94.48 & 92.34 & 93.42 \\
\hline
\end{tabular}


Table 7.1.3. Average of the parameter estimates and average of the coverage rate from the simulation study under $\lambda=1.35$.

\begin{tabular}{c|ccccc|ccccc}
\hline$\lambda=1.35$ & \multicolumn{5}{|c|}{$\theta$} & \multicolumn{5}{c}{$C R(\theta)$} \\
\hline$n$ & $\beta_{0}$ & $\beta_{1}$ & $\beta_{2}$ & $\sigma$ & $\lambda$ & $\beta_{0}$ & $\beta_{1}$ & $\beta_{2}$ & $\sigma$ & $\lambda$ \\
\hline 100 & 1.02 & 0.50 & 1.99 & 0.56 & 1.88 & 88.72 & 90.44 & 89.68 & 84.90 & 92.34 \\
200 & 1.00 & 0.50 & 2.00 & 0.58 & 1.40 & 92.48 & 93.92 & 93.18 & 91.24 & 93.76 \\
300 & 1.00 & 0.49 & 2.00 & 0.59 & 1.38 & 93.60 & 94.20 & 93.88 & 92.76 & 94.08 \\
500 & 1.00 & 0.50 & 2.00 & 0.59 & 1.36 & 93.82 & 94.38 & 93.72 & 93.58 & 94.48 \\
\hline
\end{tabular}

\subsubsection{Case: sglg}

The function sglg allows to fit a semi-parametric generalized log-gamma regression model, with a complete estimation of the parameters, linear and nonlinear components in the systematic part of the model, scale and shape. In addition, we provide some associated functions: envelope function and local influence functions under case-weight and response variable schemes.

Data sets of size $n=420,455$ were simulated from the model

$$
y_{i}=\beta_{1} x_{i 1}+\beta_{2} x_{i 2}+g\left(t_{i}\right)+\sigma \epsilon_{i}, \text { for } i=1, \cdots, n,
$$

where $x_{1} \sim \operatorname{Bernoulli}(0.5), x_{2} \sim \operatorname{Unif}(0,1), g(t)=0.4 \sin (\pi t), \sigma>0$ and $\epsilon \sim \operatorname{GLG}(0,1, \lambda)$. The values of the parameters were $\beta_{1}=0.5, \beta_{2}=2$, $t \in\{0,0.166,0.333,0.5,0.666,0.833,1\}, \sigma=0.6$ and $\lambda=-1,0.6,1$.

The number of replicates was $\mathrm{R}=2000$, keeping the values of $x_{1}$ and $x_{2}$ fixed. The summary measures considered are given below.

Mean of the estimates

$$
\hat{\theta}=\frac{1}{R} \sum_{j=1}^{R} \hat{\theta}^{j}
$$

where $\hat{\theta}^{j}$ denotes the estimated value of the parameter estimate in the $j$ th replication. Coverage rate based on a $95 \%$ confidence interval

$$
C R(\theta)=\frac{100}{R} \sum_{j=1}^{R} \mathrm{I}\left[\hat{\theta}^{j}-1.96\left[\widehat{\operatorname{Var}}\left(\hat{\theta}^{j}\right)\right]^{\frac{1}{2}}<\theta<\hat{\theta}^{j}+1.96\left[\widehat{\operatorname{Var}}\left(\hat{\theta}^{j}\right)\right]^{\frac{1}{2}}\right] .
$$

Coverage rate of the non-parametric component $g(t)$ :

$$
C R(g)=\frac{100}{R} \sum_{i=1}^{R} \prod_{j=1}^{7} \mathrm{I}\left[\hat{g}\left(t_{j}\right)-z_{\alpha^{\prime}}\left[\widehat{\operatorname{Var}}\left(\hat{g}\left(t_{j}\right)\right)\right]^{\frac{1}{2}}<g\left(t_{j}\right)<\hat{g}\left(t_{j}\right)+z_{\alpha^{\prime}}\left[\widehat{\operatorname{Var}}\left(\hat{g}_{\left(t_{j}\right)}\right]^{\frac{1}{2}}\right]\right.
$$


where $\hat{g}\left(t_{j}\right), \widehat{\operatorname{Var}}\left\{\hat{g}\left(t_{j}\right)\right\}$ are respectively, the estimates of $g\left(t_{j}\right)$ and $\operatorname{Var}\left\{\hat{g}\left(t_{j}\right)\right\}$ in the $i$-th replication and $z_{\alpha^{\prime}}=\Phi^{-1}(0.9964)$.

The results of the simulation study are summarized in Table 7.1.4. We may notice indication of consistency of the parameter estimates and good coverage rates for the parametric components as well as the empirical simultaneous intervals for the non-parametric components for large $n$. However, there is some indication that the nominal level used to construct the confidence band for $g(t)$ seems to be conservative.

Table 7.1.4. Average of the parameter estimates and average of the coverage rates for the parametric and non-parametric components from the simulation study under $\lambda=-1,0.6,1$.

\begin{tabular}{|c|c|c|c|c|c|c|c|c|c|}
\hline & \multicolumn{4}{|c|}{$\theta$} & \multicolumn{4}{|c|}{$C R(\theta)$} & $C R(g)$ \\
\hline$n$ & $\beta_{1}$ & $\beta_{2}$ & $\sigma$ & $\lambda$ & $\beta_{1}$ & $\beta_{2}$ & $\sigma$ & $\lambda$ & $g$ \\
\hline \multicolumn{10}{|l|}{$\lambda=1$} \\
\hline 420 & 0.50 & 2.01 & 0.59 & 1.05 & 93.80 & 94.05 & 89.40 & 92.80 & 99.80 \\
\hline 455 & 0.50 & 2.01 & 0.59 & 1.04 & 94.25 & 94.60 & 90.25 & 92.70 & 99.80 \\
\hline \multicolumn{10}{|c|}{$\lambda=0.6$} \\
\hline 420 & 0.50 & 2.00 & 0.59 & 0.62 & 94.50 & 93.45 & 88.55 & 92.30 & 99.90 \\
\hline 455 & 0.50 & 2.00 & 0.59 & 0.62 & 94.25 & 94.60 & 90.75 & 93.40 & 99.95 \\
\hline \multicolumn{10}{|c|}{$\lambda=-1$} \\
\hline 420 & 0.50 & 2.00 & 0.59 & -1.03 & 93.80 & 93.95 & 90.70 & 92.40 & 99.85 \\
\hline 455 & 0.50 & 2.00 & 0.59 & -1.02 & 94.20 & 94.55 & 90.95 & 92.75 & 99.75 \\
\hline
\end{tabular}

\subsubsection{Case: survglg}

The function survglg allows to fit a generalized log-gamma regression model under the presence of right censored data, with estimation of the parameters, location and scale. We give the possibility to obtain the shape parameter with the auxiliary function shape.parameter.

Data sets of size $n=200$ were generated from the model

$$
y_{i}=\beta_{1} x_{i 1}+\beta_{2} x_{i 2}+\sigma \epsilon_{i} \text { for } i=1, \cdots, n,
$$

where $x_{1} \sim \operatorname{Bernoulli}(0.5), x_{2} \sim \operatorname{Unif}(0,1), \sigma>0$ and $\epsilon \sim \operatorname{GLG}(0,1, \lambda)$. The values of the parameters were $\beta_{1}=0.5, \beta_{2}=2, \sigma \in\{0.5,1,2.5\}$ and $\lambda \in$ $\{0.5,1,2.5\}$. The number of replicates was $\mathrm{R}=5000$, keeping the values of $x_{1}$ 
and $x_{2}$ and $\lambda$ fixed. The summary measures are the same we considered in section 7.1.1.

The censoring times in Table 7.1.5 were generated from the Weibull distribution with parameters $(1.1,14),(0.6,14)$.

Table 7.1.5. Average of the parameter estimates and coverage rates for the parameters with random censoring percentage from the simulation study with sample size $n=200$ under values, $\sigma=0.5,1,2.5$, and $\lambda=1$.

\begin{tabular}{ccc|ccc|c}
\hline \multicolumn{3}{c|}{$\theta$} & \multicolumn{3}{|c|}{$C R(\theta)$} & Cens \\
\hline$\beta_{1}$ & $\beta_{2}$ & $\sigma$ & $\beta_{1}$ & $\beta_{2}$ & $\sigma$ & $\%$ \\
\hline 0.499 & 1.997 & 0.496 & 0.948 & 0.943 & 0.937 & 19.15 \\
0.497 & 1.997 & 0.495 & 0.941 & 0.942 & 0.933 & 32.36 \\
\hline \hline 0.497 & 1.995 & 0.993 & 0.948 & 0.946 & 0.939 & 19.68 \\
0.496 & 1.995 & 0.991 & 0.950 & 0.944 & 0.936 & 30.81 \\
\hline \hline 0.498 & 1.998 & 2.492 & 0.953 & 0.947 & 0.942 & 19.74 \\
0.497 & 1.991 & 2.487 & 0.950 & 0.952 & 0.937 & 31.06 \\
\hline
\end{tabular}

The censoring times in Table 7.1.6 were generated from a Weibull $(1.2,14)$ and Weibull $(0.5,14)$ for $\sigma=0.5$, and from a Weibull $(0.5,14)$ and Weibull $(0.25,14)$ for $\sigma=2.5$.

Table 7.1.6. Average of the parameter estimates and coverage rates for the parameters from the simulation study under values of $\sigma$ and $\lambda$ such that $\sigma=\lambda$.

\begin{tabular}{|c|c|c|c|c|c|c|c|}
\hline & \multicolumn{3}{|c|}{$\theta$} & \multicolumn{3}{|c|}{$C R(\theta)$} & Cens \\
\hline Parameters & $\beta_{1}$ & $\beta_{2}$ & $\bar{\sigma}$ & $\overline{\beta_{1}}$ & $\overline{\beta_{2}}$ & $\sigma$ & $\%$ \\
\hline \multirow[t]{2}{*}{$\lambda=0.5, \sigma=0.5$} & 0.500 & 1.995 & 0.497 & 0.948 & 0.946 & 0.940 & 19.56 \\
\hline & 0.500 & 1.994 & 0.496 & 0.942 & 0.945 & 0.937 & 31.07 \\
\hline \multirow[t]{2}{*}{$\lambda=2.5, \sigma=2.5$} & 0.477 & 1.962 & 2.486 & 0.943 & 0.941 & 0.940 & 20.49 \\
\hline & 0.468 & 1.937 & 2.478 & 0.940 & 0.939 & 0.940 & 30.08 \\
\hline
\end{tabular}

From the simulation studies described in Tables 7.1.5 and 7.1.6 we may notice indication of consistency for the parameters estimates as well as increasing coverage rates for large $n$. 


\subsubsection{Case: ssurvglg}

The function ssurvglg allows to fit a semi-parametric generalized log-gamma regression model with right censored data, with estimation of the parameters of location and scale, linear and nonlinear components in the systematic part of the model.

Data sets of size $n=350$ were generated from the model

$$
y_{i}=\beta_{1} x_{i 1}+\beta_{2} x_{i 2}+g(t)+\sigma \epsilon_{i} \text { for } i=1, \cdots, n,
$$

where $x_{1} \sim \operatorname{Bernoulli}(0.5), x_{2} \sim \operatorname{Unif}(0,1), g(t)=0.4 \sin (\pi t), \sigma>0$ and $\epsilon \sim \operatorname{GLG}(0,1, \lambda)$. The values of the parameters were $\beta_{1}=0.5, \beta_{2}=2$, $t \in\{0,0.166,0.333,0.5,0.666,0.833,1\}, \sigma=\{0.5,0.75\}$ and $\lambda=\{0.5,1\}$. The number of replicates was $\mathrm{R}=2000$, keeping the values of $x_{1}$ and $x_{2}$ fixed. The censoring times in Table 7.1.7 were generated from a Weibull distribution with parameters $(0.7,14)$.

Table 7.1.7. Average of the parameter estimates and average of the coverage rates for the parameter and the non-parametric component for a random censoring percentage for some values of $(\lambda, \sigma)$.

\begin{tabular}{c|ccc|ccc|c|c}
\hline & \multicolumn{3}{|c|}{$\theta$} & \multicolumn{3}{c|}{$C R(\theta)$} & Cens & $C R(g)$ \\
\hline Parameters & $\beta_{1}$ & $\beta_{2}$ & $\sigma$ & $\beta_{1}$ & $\beta_{2}$ & $\sigma$ & $\%$ & \\
\hline$\sigma=0.5, \lambda=0.5$ & 0.50 & 2.00 & 0.49 & 95.25 & 94.30 & 91.05 & 33.6 & 99.4 \\
$\sigma=0.75, \lambda=1$ & 0.50 & 2.00 & 0.74 & 95.10 & 95.25 & 92.00 & 32.6 & 97.8 \\
\hline
\end{tabular}

From the simulation studies described in Tables 7.1 .7 we may notice indication of consistence of the parameters estimates, good coverage rates for the parameter components as well as the empirical simultaneous intervals for the non-parametric components for large $n$. However, the nominal level used to construct the confidence band for $g(t)$ seems to be conservative.

\subsubsection{How to select the starting values}

It is well known that good starting values for some iterative optimization algorithm are essential. The following diagram present us the internal steps of the functions that we have developed to obtain good starting values.

In Figure 7.1 the bond arrows presents the paths with the better empirical starting values. For example, if we want to fit a semi-parametric log-gamma model we use the function sglg. The path to obtain good initial values would 


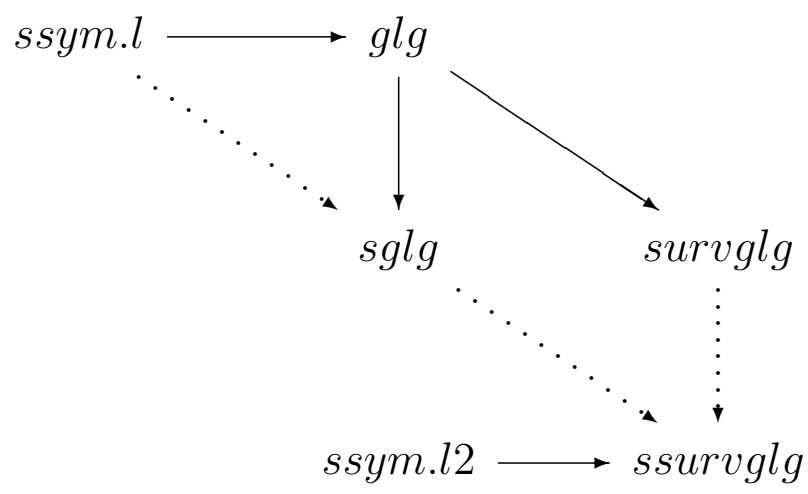

Figure 7.1: Diagram of possible selection of the starting values in the functions glg, sglg, survglg and ssurvglg.

be first to fit a normal linear model with the function ssym.l after that to fit a generalized log-gamma liner model with the function glg. Finally, we begin the process by fitting a semi-parametric generalized log-gamma model with the function sglg.

\subsection{Diagnostic related grouping system, DRG}

This data set is about 70323 stays in a group of hospitals of Switzerland in the year 2000. It is available in the object $\mathbf{d r g} 2000$ of the $\mathbf{R}$ package robustloggamma. This data belongs to an initial study with the objective of testing a diagnostic related grouping (DRG) system. This kind of systems are used in modern hospital management to classify each individual stay into a group based on the patient characteristics. Four variables were recorded:

- LOS, length of the hospitalization (in days),

- Cost, cost of the hospitalization in Switzerland franc,

- MDC, major diagnostic category, this variable has 28 categories,

- APDRG, all patient diagnostic related group.

From the 28 categories of the MDC variable, we select the category 3 , that contains 2442 observations. In the following we present an exploratory study of the variables Cost and LOS for the patients of this category. 


\subsubsection{A preliminary analysis}

We present some graphs that give us some indication on the behavior of $\log ($ Cost $)$ and its relationship with the covariate LOS. From Figure 7.2 one may notice the presence of asymmetry in the density graph of $\log (\mathrm{Cost})$. Also, we may see a possible nonlinear behavior between $\log ($ Cost $)$ versus LOS.
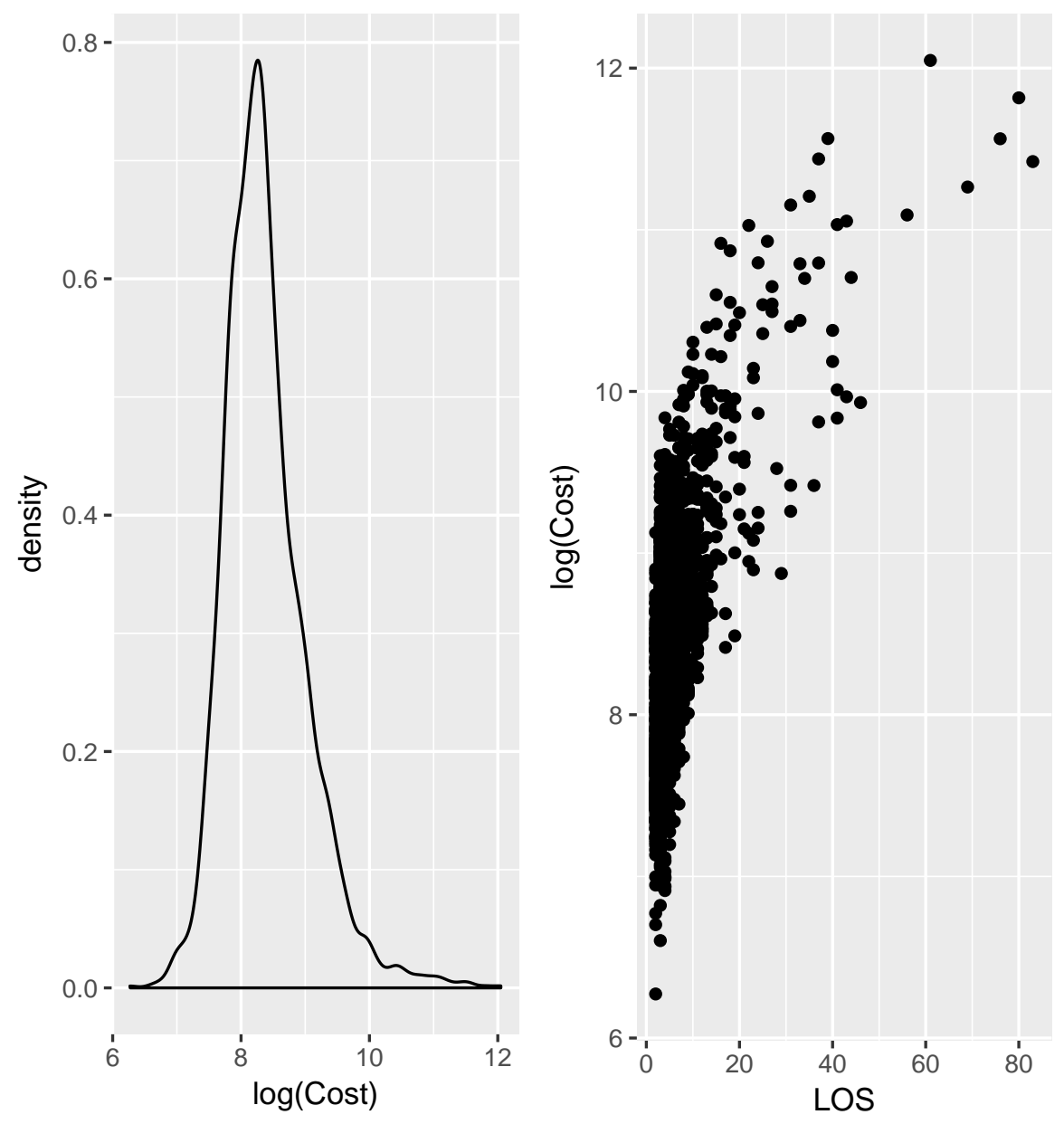

Figure 7.2: Density of $\log ($ Cost) (left) and the dispersion graph between $\log ($ Cost $)$ and LOS for the patients in category 3 (right). 


\subsubsection{The model}

Based on the graph described in Figure 7.2 we propose the following model:

$$
y_{i}=\beta_{0}+g\left(\operatorname{LOS}_{i}\right)+\sigma \epsilon_{i}
$$

where $y_{i}$ is $\log \left(\operatorname{Cost}_{i}\right)$ and Cost $_{i}$ is the cost observed for the $i$-th stay in the category 3 of the variable MRD, $\operatorname{LOS}_{i}$ is the length for the $i$-th stay, $g(\cdot)$ is a unknown smooth function approximated by P-splines and $\epsilon_{i} \sim \operatorname{GLG}(0,1, \lambda)$, for $i=1,2, \cdots, 2442$. We employ the function sglg to fit the model.

Table 7.2.1. Parameter estimates and their approximate standard errors for the parametric component of the model (7.1) fitted to the drg2000 data.

\begin{tabular}{c|r|r}
\hline Parameter & Estimate & Std Error \\
\hline Intercept & 5.330 & 0.391 \\
$\sigma$ & 0.476 & 0.007 \\
$\lambda$ & -0.382 & 0.049 \\
\hline
\end{tabular}

The parameter estimates with their approximate standard errors are given in Table 7.2.1, whereas the results on the non-parametric component are described in Table 7.2.2. The $\lambda$ estimate confirms the right-skewed distribution for the covariate LOS.

Table 7.2.2. Smoothing parameter estimate, basis dimension and effective degree of freedom for the non-parametric component.

\begin{tabular}{c|c|c|c}
\hline Covariate & Smooth parameter & Basis Dim & d.f. \\
\hline LOS & 1.342 & 16 & 6.55 \\
\hline
\end{tabular}

Table 7.2.3. Values of $\Upsilon$, AIC and SIC for some generalized log-gamma models.

\begin{tabular}{c|c|c|c}
\hline Model & $\Upsilon$ & AIC & SIC \\
\hline$\beta_{0}+$ LOS & 0.066 & 3660.78 & 3683.98 \\
$\beta_{0}+g($ LOS $)$ & 0.073 & 3442.73 & 3498.15 \\
\hline
\end{tabular}



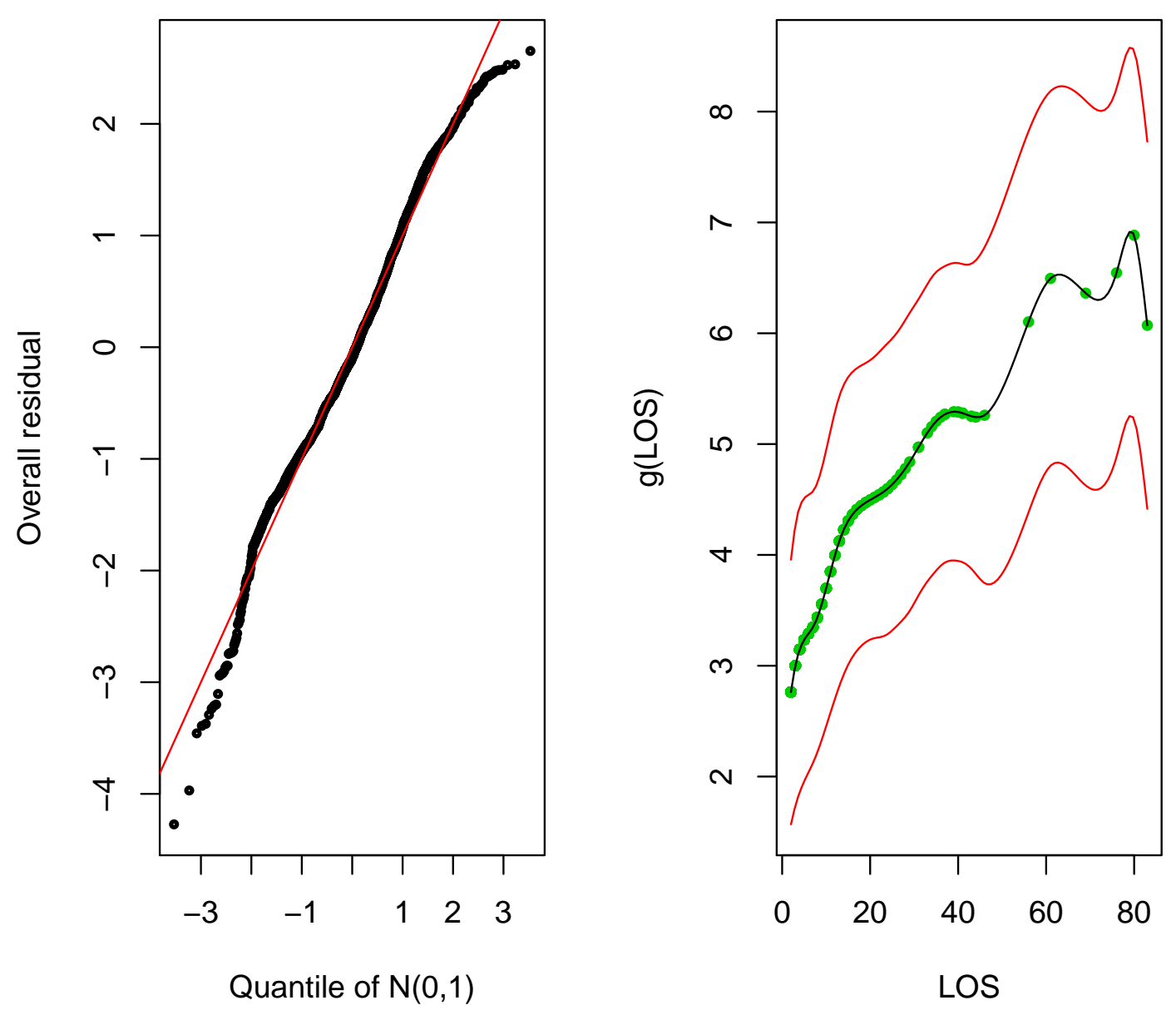

Figure 7.3: Overall goodness-of-fit statistic $\Upsilon$ (left), and the graph of $\hat{g}$ (LOS) as well as simultaneous $95 \%$ confidence intervals from the semi-parametric generalized log-gamma model (7.1) fitted to the drg200 data (right).

The basis dimension is calculated as $\min \left\{\left\lfloor n^{\frac{1}{3}}\right\rfloor+3, n_{L O S}\right\}$, where $n$ is the sample size and $n_{L O S}$ is the number of different values that the variable LOS takes in the sample. In this example, $n=2442,\left\lfloor n^{\frac{1}{3}}\right\rfloor=13$ and $n_{L O S}=46$. 


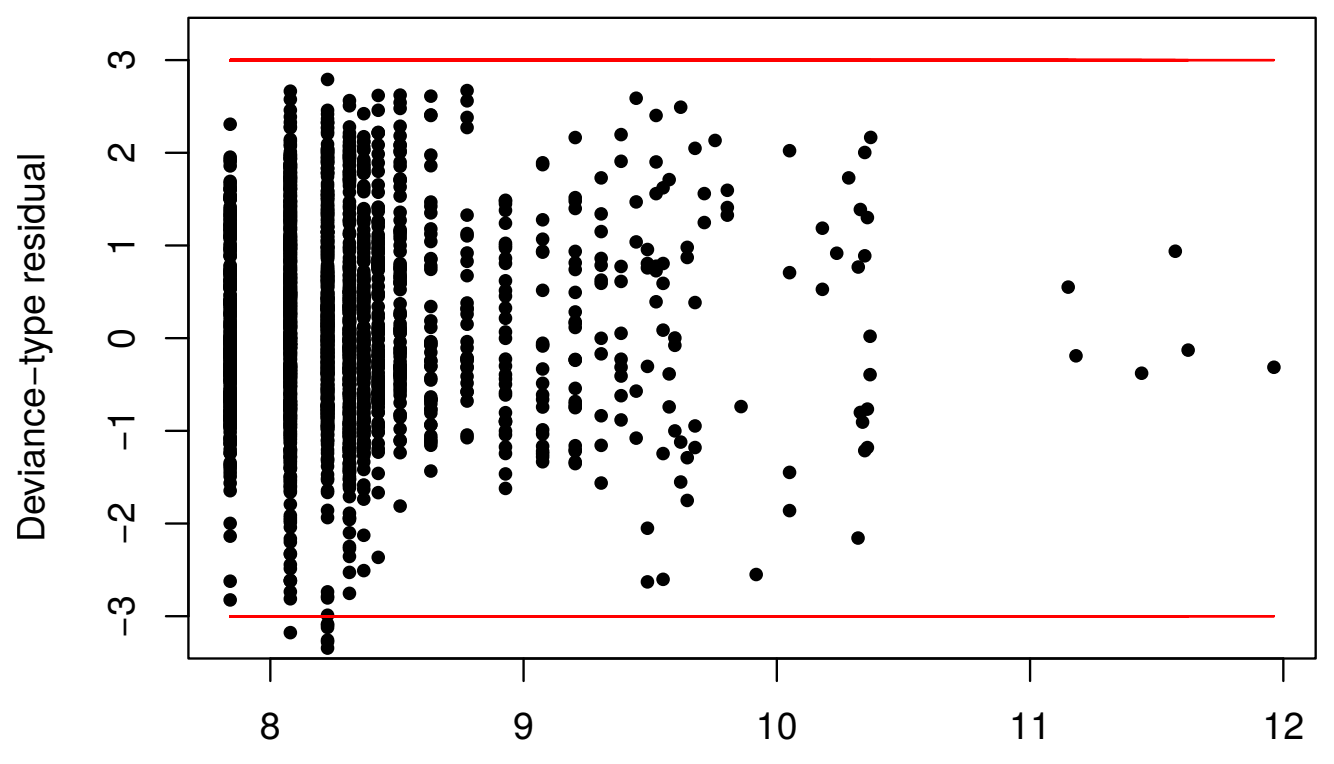

Fitted value

Figure 7.4: Dispersion graph between the deviance-type residual and the fitted value from the generalized log-gamma model fitted to the drg2000 data.

Table 7.2.3 presents the value of $\Upsilon$, AIC and SIC for the model (7.1) as well as the systematic component of the generalized log-gamma model fitted to the diagnostic data.

Figure 7.4 describes the dispersion graph between the deviance residual and the fitted value. There is not indication of no constant variance. The normal probability plot of the deviance residual is described in Figure 7.5 with the simulated confidence band of $95 \%$. We may notice a reasonable fit since some residuals appear outside the confidence band but near the bounds.

The graphs of local influence measures described in Figure 7.6 under the case-weight perturbation scheme that help us to identify the following sets of observations $\{120,126,213,240,520,1434\},\{120\},\{126\},\{213\},\{520\}$ and $\{1434\}$ as potentially influential on $\hat{\boldsymbol{\theta}}$. None inferential change was obtained when the model (7.1) was adjusted by dropping these observations.

Just for a purpose of comparison we fitted model (7.1) under some symmetric errors, as described in Table 7.2.4 and by applying the library ssym in R Vanegas and Paula (2016). The obtained AIC and SIC were larger than under the 


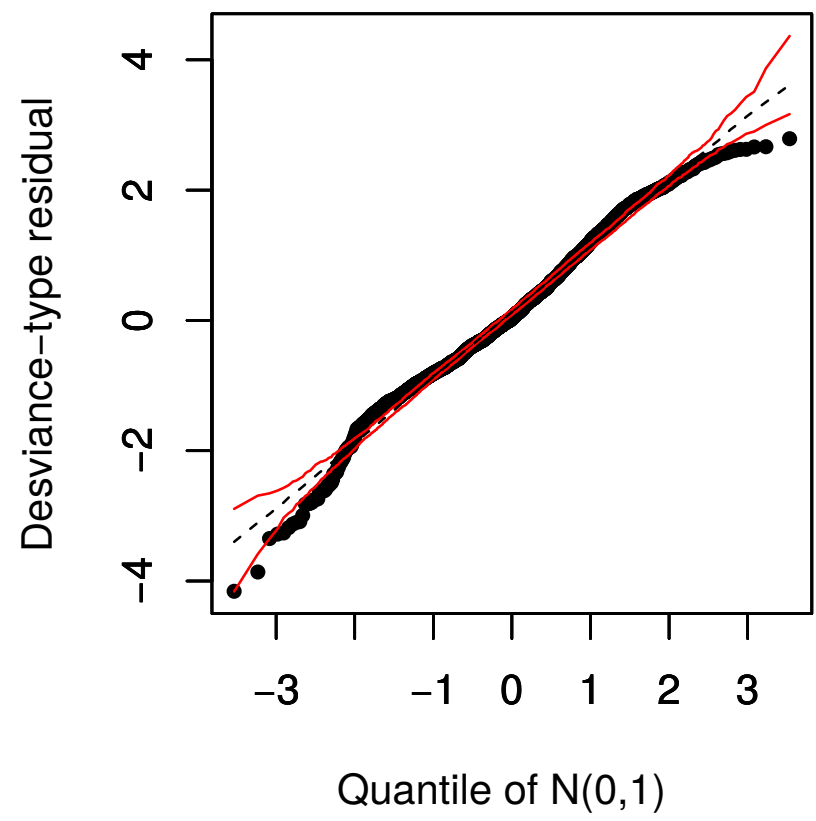

Figure 7.5: Normal probability plot of the deviance-type residual with a confidence band of $95 \%$ from the generalized log-gamma model fitted to the drg2000 data.

generalized log-gamma model (see Table 7.2.3) as well as the respective normal probability plots presented a poor behavior (omitted here). Thus, it seems that asymmetric errors are more appropriate for modelling $\log$ (Cost). Another possible comparison would be between partially linear under generalized loggamma errors models and skew-normal errors Ferreira and Paula (2017).

Table 7.2.4. Values of $\Upsilon$, AIC and SIC for some log-symmetric models.

\begin{tabular}{cccc}
\hline Error & $\Upsilon$ & AIC & BIC \\
\hline Normal & 0.1072 & 3477.839 & 3512.528 \\
Student(7.5) & 0.1007 & 3501.066 & 3535.782 \\
Slash(1.5) & 0.0944 & 3711.445 & 3728.846 \\
Power-exponential(0.25) & 0.1001 & 3482.864 & 3517.582 \\
Birbaum-Saunders(0.4,11) & 0.1034 & 3479.163 & 3513.865 \\
Hyperbolic(2.4) & 0.1004 & 3494.926 & 3529.096 \\
\hline
\end{tabular}



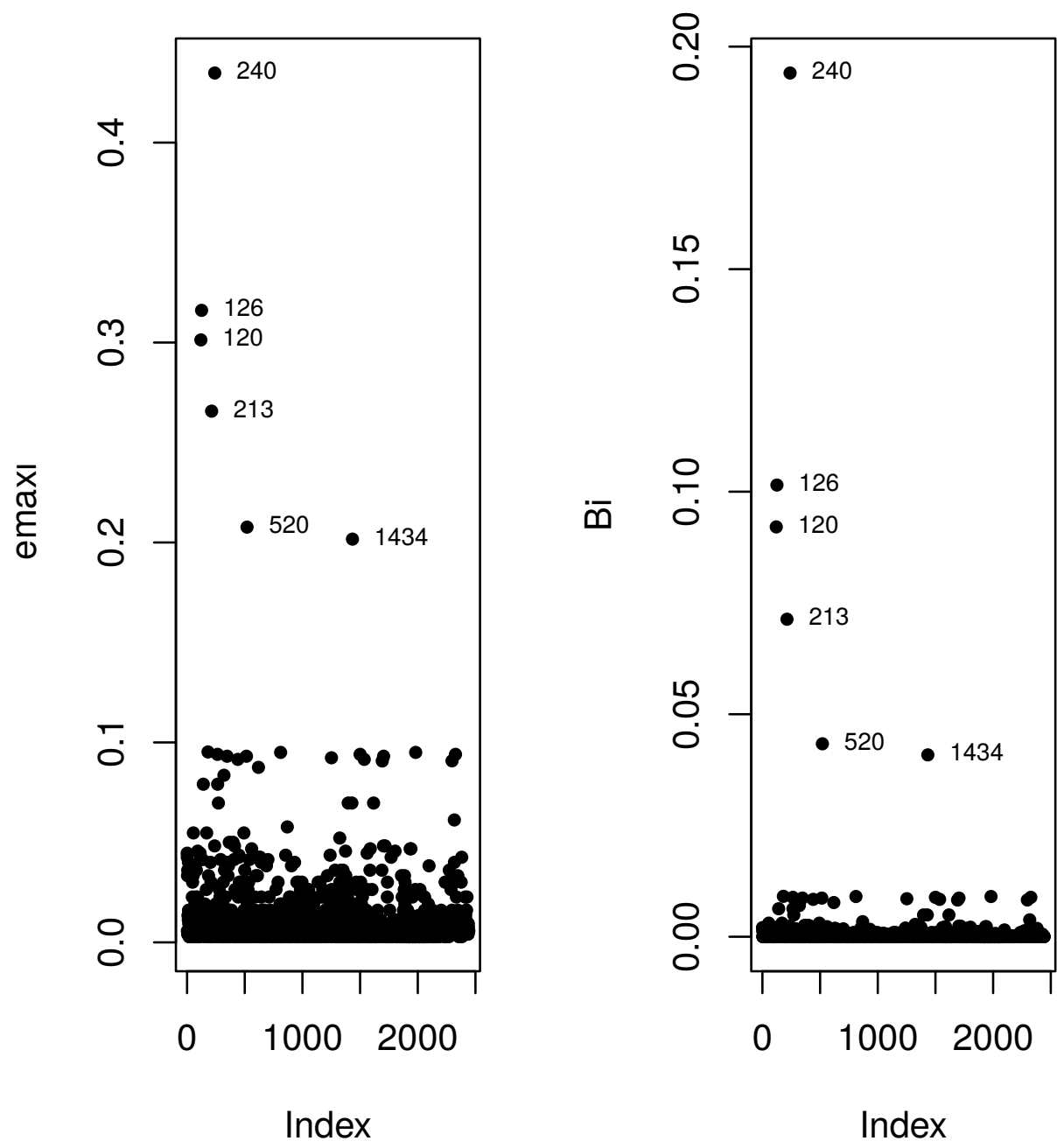

Figure 7.6: Index plot based on the contribution of individual basic perturbation $E_{i}$ to the eigenvector $e_{\max }$ which gives the maximum conformal normal curvature (left) and of $B_{i}$ (right) under the case-weight perturbation scheme from the partially linear generalized log-gamma model fitted to the drg2000 data.

\subsection{Primary biliary cirrhosis}

In this section, as illustration, we will analyze a data set available in the $\mathrm{R}$ package survival, called pcb. This was a Mayo Clinic trial in primary biliary cirrhosis (PBC) of the liver carried out from 1974 to 1984. After to discard the 
individuals with missing values the data consist of 412 observations.

We have the response variable, time, that denotes the number of days between registration and the earliest of death, transplantation, or study analysis in July, 1986; and other independent variables, such as status (0: alive at the last contact, 1: liver transplant, 2: death), edema (0: no edema, 0.5: edema present without diuretics or edema resolved by diuretics, 1: edema despite diuretic therapy), stage: histological stage of disease; and bili: level of serum bilirubin $(m g / d l)$. By considering the status condition "alive at last contact" as censoring, one has $55.83 \%$ of right-censored data.

\subsubsection{A preliminary analysis}

In the following we present some graphs that give us indication of the relationship between $\log$ (time) and the covariates edema, age, and bili. From Figure 7.7 one may notice the presence of asymmetry in the density graph of the $\log$ (time) response and in the boxplots of $\log$ (time) according with the scheme levels the $\log$ (time) tends to decrease as the stage increases and we may see a possible nonlinear behavior between $\log$ (time) and bili.

\subsubsection{The model}

Similarity to Vanegas and Paula (2017) we propose the following model:

$$
y_{i}=\beta_{0}+\beta_{1} * \operatorname{edema} 0.5_{i}+\beta_{2} * \operatorname{edema1}_{i}+\beta_{3} * \operatorname{stage}_{i}+g\left(\operatorname{Bili}_{i}\right)+\sigma \epsilon_{i},
$$

where $y_{i}$ denotes the $\log \left(\right.$ time $\left._{i}\right)$ for the $i$ th individual, edema 0.5 and edema 1 are dummy variables and $g(\cdot)$ is a unknown smooth function and $\epsilon_{i} \sim \operatorname{GLG}(0,1, \lambda)$, for $i=1, \cdots, 412$. We employ the function ssurvglg with P-spline option to fit this model.

Table 7.3.1 presents the parameter estimates and their approximated standard errors for the parametric components. 
Table 7.3.1. $\quad$ Parameter estimates and their approximated standard errors from the fit of the semi-parametric generalized log-gamma model to the PBC data.

\begin{tabular}{c|r|r|r}
\hline Effect/Parameter & Estimate & Std Error & z-value \\
\hline Intercept & 15.780 & 1.944 & 8.12 \\
Edema 0.5 & -0.509 & 0.154 & -3.30 \\
Edema 1 & -1.082 & 0.205 & -5.27 \\
Stage & -0.376 & 0.072 & -5.20 \\
$\sigma$ & 0.795 & 0.050 & 15.69 \\
\hline
\end{tabular}

We may notice from Table 7.3.1 that the expected survival time seems to be smaller for patients with Edema 0.5 and Edema 1 with respect to patients with no Edema. Also, the expected survival time seems to decrease as the stage increases. To obtain the shape parameter $\lambda$ we minimize the overall goodnessof-fit criterion $\Upsilon$. In this case, we obtain $\hat{\lambda}=0.51$ with $\Upsilon=0.0435$. The $\lambda$ estimate confirms the left-skewed distribution for the variable $\log$ (time).

Table 7.3.2. Smoothing parameter estimate, basis dimension and effective degrees of freedom for the non-parametric component.

\begin{tabular}{c|c|c|c}
\hline Covariate & Smooth parameter & Basis Dim & d.f. \\
\hline bili & 5.05 & 10 & 6.78 \\
\hline
\end{tabular}

Table 7.3.3. Overall goodness-of-fit for some log-symmetric models fitted to the PBC data set.

\begin{tabular}{c|c}
\hline Model & $\Upsilon$ \\
\hline Log-normal & 0.103 \\
Log-Student-t(6) & 0.071 \\
Log-hyperbolic $(1.04)$ & 0.067 \\
Log-power-exponential $(0.47)$ & 0.071 \\
Log-contaminated-normal $(0.38,0.2)$ & 0.067 \\
Birnbaum-Saunders $(0.05)$ & 0.103 \\
\hline
\end{tabular}


Density of log(time)
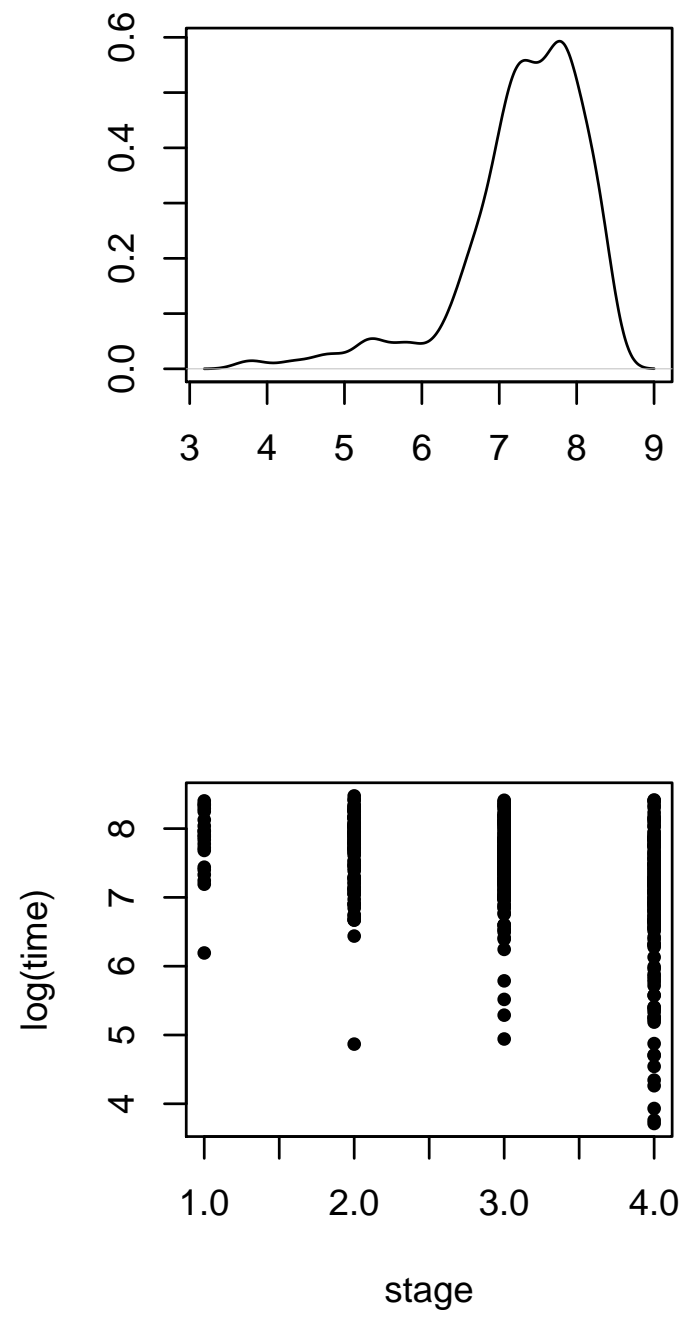

Boxplot log(time)-edema
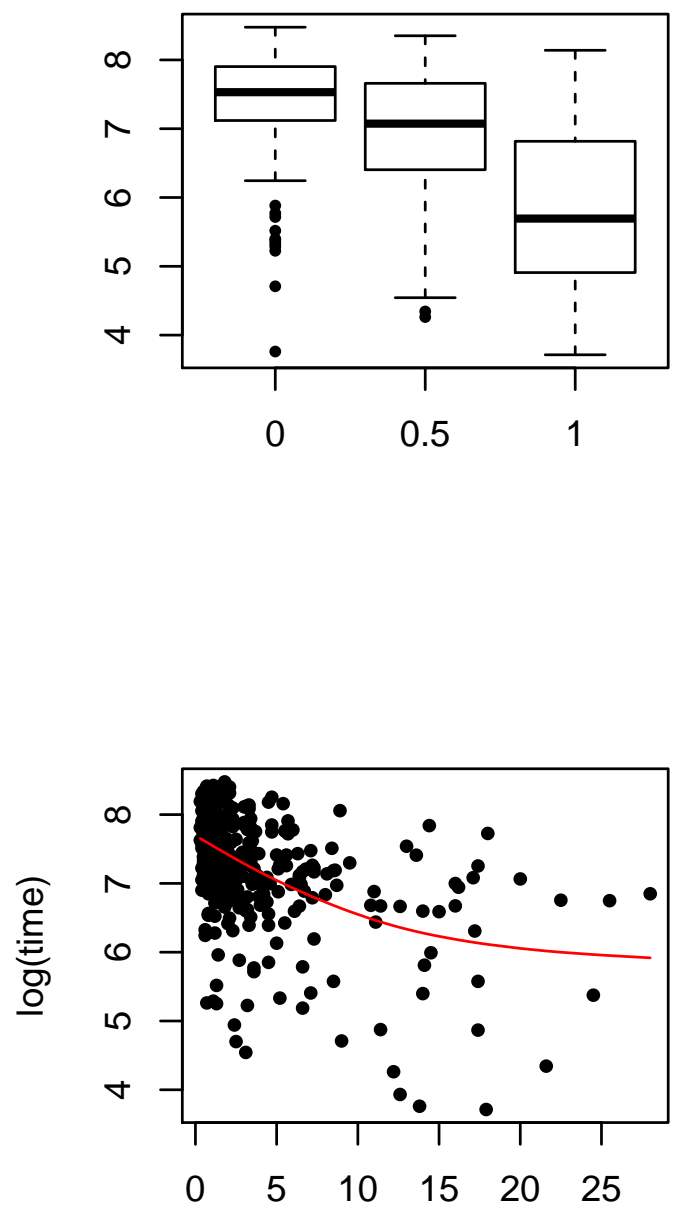

Bili

Figure 7.7: Behaviour of $\log ($ time) and relationship with edema, stage and bili covariates.

The basis dimension is calculated as $\min \left\{\left\lfloor n^{\frac{1}{3}}\right\rfloor+3, n_{b i l i}\right\}$, where $n$ is the sample size and $n_{\text {bili }}$ is the number of different values that the variable bili takes in the sample. In this example, $n=412,\left\lfloor n^{\frac{1}{3}}\right\rfloor=7$ and $n_{\text {bili }}=98$. 
Table 7.3.3 describes the value of the overall goodness-of-fit statistic for some log-symmetric regression models (see Vanegas and Paula, 2017) fitted to the $\mathrm{PBC}$ data set. We may notice that the fitted semi-parametric generalized loggamma regression model has the minimum $\Upsilon$ value. This implies that it presents the best overall goodness-of-fit of the PBC data when compared with some logsymmetric models. Its AIC and SIC values are 690 and 737, respectively. Figure 7.8 describes the estimated survival function and the normal probability plot for the overall residual, which confirms the suitable fit to the PBC data.

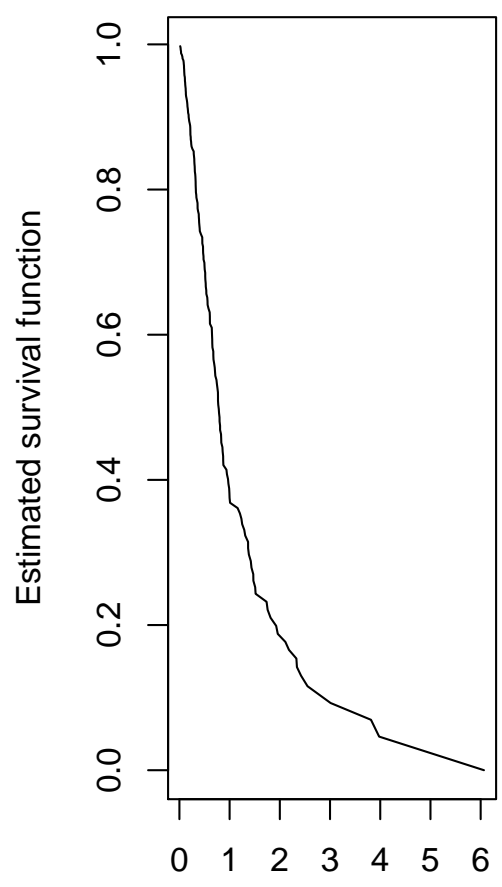

Survival time

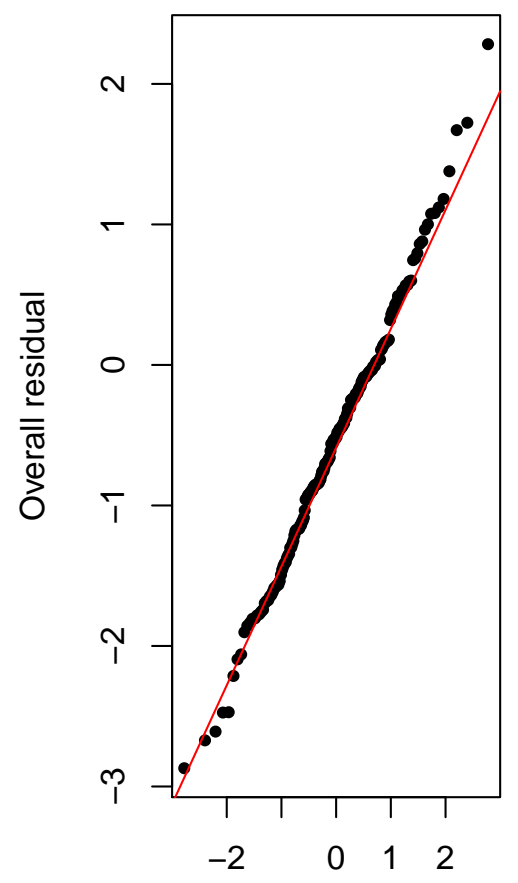

Quantile of $\mathrm{N}(0,1)$

Figure 7.8: Estimated survival function (left) and the normal probability plot for the overall residual (right).

The graphs of local influence measures described in Figure 7.9 under the case-weight perturbation scheme that help us to identify the following sets of 
observations $\{44,293\}$ and $\{63\}$ as potentially influential on $\hat{\boldsymbol{\theta}}$. None inferential change was obtained when the model (7.2) was adjusted by dropping these observations.
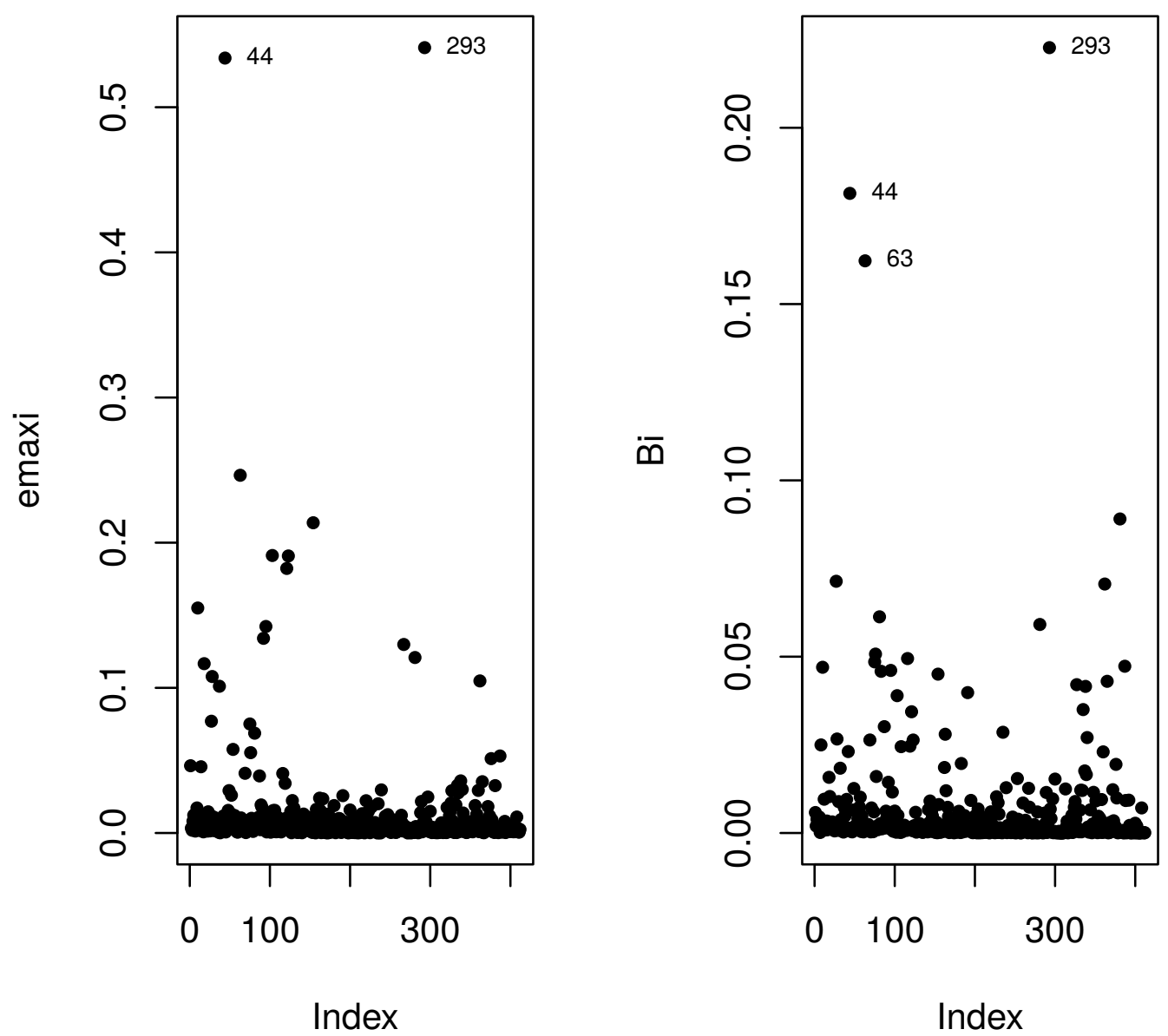

Figure 7.9: Index plot based on the contribution of individual basic perturbation $E_{i}$ to the eigenvector $e_{\max }$ which gives the maximum conformal normal curvature (left) and of $B_{i}$ (right) under the case-weight perturbation scheme from the partially linear generalized log-gamma model under the presence of censored observations adjusted to the PBC data. 


\section{Chapter 8}

\section{Conclusions}

\subsection{Final considerations}

In this thesis we first presented a revision of the generalized log-gamma distribution. We proposed to fit partially linear models with generalized log-gamma errors, in which the non-parametric component may be expressed in a linear form. The particular cases of natural cubic splines and P-splines, which use different penalization in the log-likelihood functions, are considered. Extensions for the additive case are presented with a back-fitting algorithm being developed for the uncensored case. Some asymptotic results are also performed in order to derive a consistent variance-covariance estimator for the parametric and non-parametric estimators.

Usual diagnostic procedures, such as deviance-type residuals and overall residuals are derived for the uncensored and censored cases, and the local influence curvatures are derived for two perturbation schemes. Simulation studies are performed to assess the empirical distributions of the parametric and semiparametric estimators and two real data sets with uncensored and censored observations are analyzed by the procedures developed in the work. Finally, a library named sglg was developed in $\mathbf{R}$ to fit semi-parametric generalized log-gamma regression models with uncensored and censored observations.

\subsection{Future works}

Several next steps are possible for the present work. In the following some of them: 
- Joint modeling the location and dispersion parameters in a semi-parametric way.

- Assuming other patterns of censoring and truncation, such as left truncated at $w$ and observed at $t>w$, left truncated at $w$ and rigth censored at $t$, interval censored between $t_{1}$ and $t_{2}$.

- Exploring more properties of the generalized log-gamma distribution. 


\section{Appendix A}

\section{Cubic splines and P-splines}

When we are working with partially linear or additive models we need to consider ways to estimate the non-parametric components in the model. In our work we had chosen to particularize the fitting through the natural cubic splines and P-splines.

\section{A.1 Natural Cubic Splines}

Let be $\left[k_{1}, k_{n}\right]$ a real finite interval and $k_{1}<k_{2}<\cdots<k_{n-1}<k_{n}$ a partition of it. A polynomial spline on $\left[k_{1}, k_{n}\right]$ is a function building with polynomial sections between $\left[k_{i}, k_{i+1}\right]$ with $i=1, \cdots, n-1$. In its case the sections are cubic and we may say more, the first and the last sections are linear. A key point between one section and other is the way that they are joined, which is made in a smooth form. That means

$$
\begin{aligned}
& p_{i}\left(k_{j}\right)=p_{i+1}\left(k_{j}\right), \\
& p_{i}^{\prime}\left(k_{j}\right)=p_{i+1}^{\prime}\left(k_{j}\right) \text { and } \\
& p_{i}^{\prime \prime}\left(k_{j}\right)=p_{i+1}^{\prime \prime}\left(k_{j}\right),
\end{aligned}
$$

where $p_{i}(\cdot)$ is the $i$ th cubic section with $i=1, \cdots, n-2$ and $j=2, \cdots, n-1$. The following key properties behind the cubic spline make them so appealing for penalized regression. 


\section{A.2 Some properties}

Let $H=C^{2}\left[k_{1}, k_{n}\right]$ be the space of all functions with second derivative continuous. If $g \in H$, there is possible to define several measures of the roughness $J(\cdot)$ of $g(\cdot)$ which will be a non-negative functional on $H$. The minimal conditions over that measures are

$$
\begin{aligned}
J(g) & <\infty, \\
J(\cdot) & \text { is a seminorm and } \\
\operatorname{dim}(\operatorname{Ker}(J)) & <\infty .
\end{aligned}
$$

$\operatorname{Ker}(J)$ means the null space of $J$, and $\operatorname{dim}(\cdot)$ is the dimension, see Gu and Kim (2002).

One traditional choice is

$$
J_{2}(g)=\int_{k_{1}}^{k_{n}}\left[g^{\prime \prime}(t)\right]^{2} d t .
$$

It is relatively simply to see that $J_{2}$ satisfies the conditions,

$$
\begin{aligned}
J_{2}(g) & \geq 0, \text { for all } g \in \mathrm{H}, \text { by definition, } \\
J_{2}(g) & \leq(b-a) \mathrm{M}, \text { with } \mathrm{M}=\sup _{t \in[a, b]}\left[g^{\prime \prime}(t)\right]^{2} \text { and } \\
\operatorname{Ker}\left(J_{2}\right) & =p(t): p(x)=a+b t, \text { the subspace of lineal polynomials. }
\end{aligned}
$$

Suppose we want to interpolate the set $\left(k_{1}, y_{1}\right), \cdots,\left(k_{n}, y_{n}\right)$ and let $g(\cdot)$ the natural cubic spline defined by these points. Then it is possible to show that $g(\cdot)$ is the smoothest interpolator with respect of $J_{2}$, that is, $J_{2}(g) \leq J_{2}(f)$ for all $f \in H$. Therefore, $g$ is the best interpolating.

From the statistical perspective, $y_{i}$ is often measured with error, and because of this it is more useful to smooth the set $\left(x_{1}, y_{1}\right), \cdots,\left(x_{n}, y_{n}\right)$. So, if we minimize

$$
\sum_{i=1}^{n}\left\{y_{i}-f\left(x_{i}\right)\right\}^{2}+\alpha \int_{x_{1}}^{x_{n}}\left[f^{\prime \prime}(x)\right]^{2} d x
$$

in $H$, we will find that $g(\cdot)$ achieves this goal and is the unique. Therefore, $g$ is the best smoothing. This result was first derived by Reinsch (1967). 


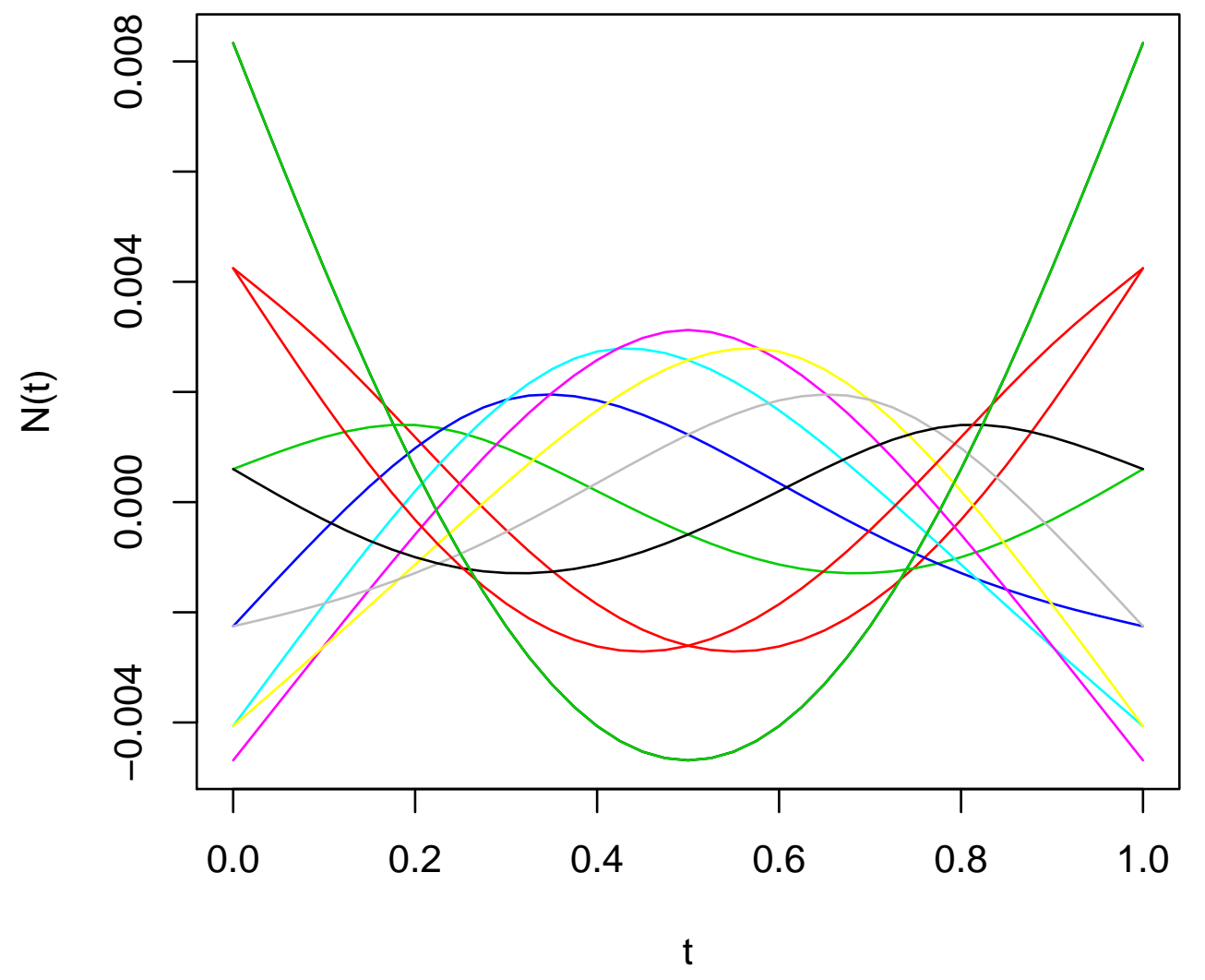

Figure A.1: An example of Gu's natural cubic spline base with knots in $0,0.1,0.2,0.3,0.4,0.5,0.6,0.7,0.8,0.9$.

We work with the following natural cubic spline basis, Gu and Kim (2002),

$$
\begin{aligned}
N_{j}(t) & =\frac{1}{4}\left\{\left(k_{j}-\frac{1}{2}\right)^{2}-\frac{1}{12}\right\}\left\{\left(t-\frac{1}{2}\right)^{2}-\frac{1}{12}\right\} \\
& -\frac{1}{24}\left\{\left(\left|t-k_{j}\right|-\frac{1}{2}\right)^{4}-\frac{1}{2}\left(\left|t-k_{j}\right|-\frac{1}{2}\right)^{2}+\frac{7}{240}\right\},
\end{aligned}
$$

where $j \in\{1, \cdots, q\}$ and $k_{1} \leq t \leq k_{q}$. The next figure shows how looks a Gu's base. 


\section{A.3 P-splines}

P-splines were proposed by Eilers and Marx (1996) and their central goal was to simplify the choosing of the optimal number and positions of the knots when we work with B-splines. One feature of B-spline is that are strictly local. Eilers and Marx proposed to change the functional $J_{2}(\cdot)$ defined in the last subsection for difference penalties on the coefficients of adjacent B-splines. They showed that the behavior for second order difference is similar. But, the new advantage is that in some applications is better to employ differences of a smaller or high order in the penalty.

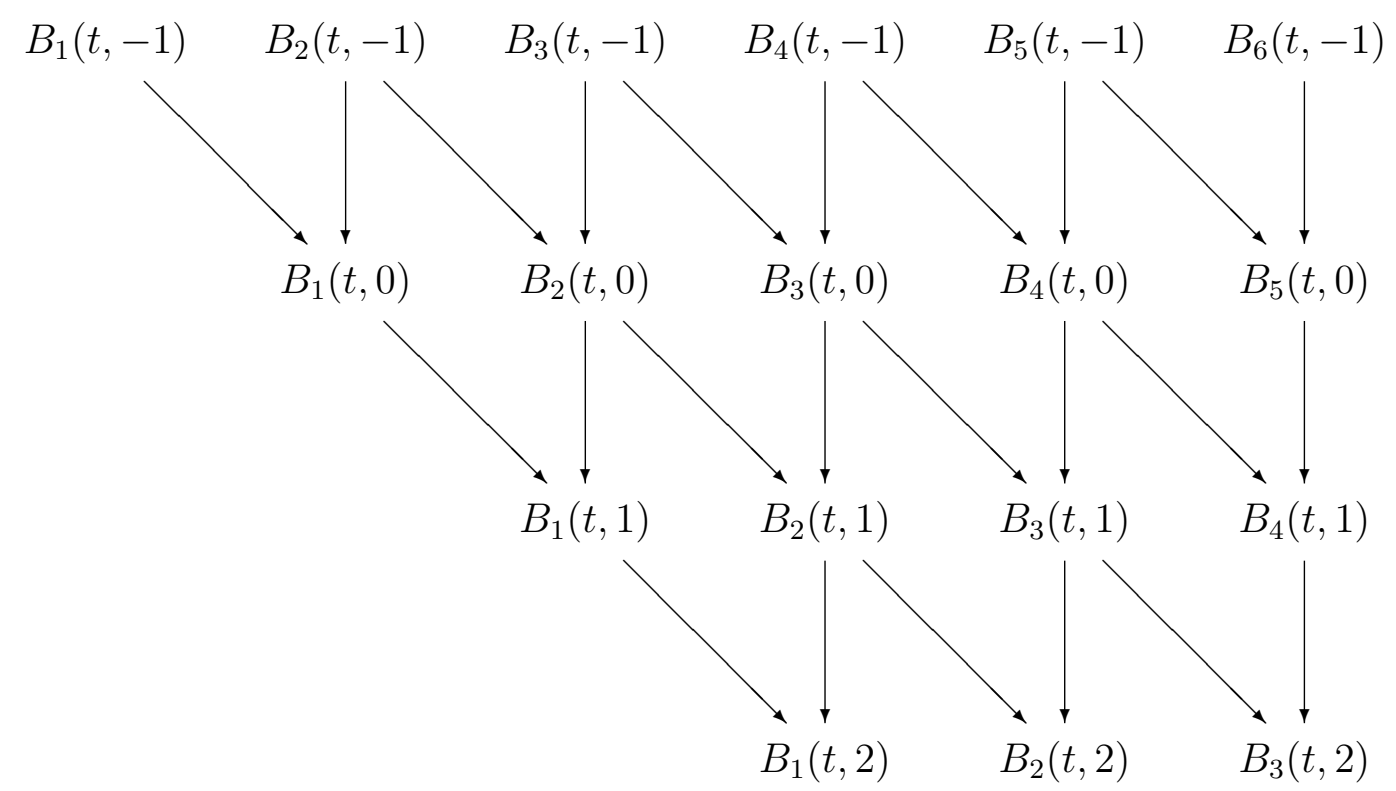

The way we build the B-splines comes from Wood (2006). If we want to build $q$ B-splines of order $o=m+1$ in one interval we need $q+o+2$ knots. The basis functions are defined as follows

$$
B_{j}(t, m)=\frac{\left(t-k_{j}\right)}{k_{j+m+1}-k_{j}} B_{j}(t, m-1)+\frac{\left(k_{j+m+2}-t\right)}{k_{j+m+2}-k_{j+1}} B_{j+1}(t, m-1)
$$

with $m \geq 0,1 \leq j \leq q$ and

$$
B_{j}(t,-1)=\left\{\begin{array}{lc}
1 & k_{j} \leq t<k_{j+1} \\
0 & \text { otherwise }
\end{array}\right.
$$


We can visualize the process in the following diagram. For this example we work with $m=2, k=3$. Therefore, we need $m+k+2=6$ starting with B-splines of order 0 .

The arrows in the diagram indicate us how one particular basis functions $B_{j}(t, 2)$ depends from the basis functions with lower order. It also gives a clearly idea of how the effective interval of definition (the interval where is not zero) changes through the different levels.

Then, a $3 t h$ order spline can be represented as

$$
f(t)=\sum_{j=1}^{n} B_{j}(t, 2) \gamma_{j} .
$$

Some general properties of a B-spline of order $m+1$ :

1. It is formed by $m+2$ polynomial sections, each of degree $m+1$.

2. The polynomial sections joint at $m+1$ inner knots.

3. Except at the boundaries, it overlaps with $2(m+1)$ polynomial sections of its neighbors.

4. At the knots, derivatives up to order $m$ are continuous.

5. Given a point $x$ different of the knots, $m+2$ B-splines are non-zero.

The other essential ingredient in the P-splines approach is the Difference Penalties, which applies to the $\gamma_{j}$ with the objective to control the roughness of the function

$$
P_{k}(\gamma)=\sum_{j=k+1}^{n}\left(\Delta^{k} \gamma_{j}\right)^{2}
$$

where

$$
\Delta^{k} \gamma_{j}=\sum_{i=0}^{k}(-1)^{i}\left(\begin{array}{c}
k \\
i
\end{array}\right) \gamma_{j-i}
$$

We are interested principally in $k=2$

$$
\Delta^{2} \gamma_{j}=\gamma_{j}-2 \gamma_{j-1}+\gamma_{j-2}
$$

which can be written in matrix form as

$$
P_{2}(\gamma)=\gamma^{\top} \mathbf{D}^{\top} \mathbf{D} \gamma
$$


where

$$
\mathbf{D}=\left[\begin{array}{cccccc}
-1 & 1 & 0 & 0 & 0 & \cdots \\
0 & -1 & 1 & 0 & 0 & \cdots \\
0 & 0 & 0 & -1 & 1 & \cdots \\
\vdots & \vdots & \vdots & \vdots & \vdots &
\end{array}\right]
$$

and

$$
\mathbf{M}=\mathbf{D}^{\top} \mathbf{D}=\left[\begin{array}{cccccc}
1 & -2 & 1 & 0 & 0 & \cdots \\
0 & 1 & -2 & 1 & 0 & \cdots \\
0 & 0 & 1 & -2 & 1 & \cdots \\
\vdots & \vdots & \vdots & \vdots & \vdots &
\end{array}\right]
$$

Remark. P-splines do not only give another way to estimate the non-parametric component of the model but also open the possibility to apply in other situations. Note that $\mathbf{D}$ here denotes a completely different thing to the $\mathbf{D}$ of chapter 3. For more refinements about splines see de De Boor (1978); Green and Silverman (1994). 


\section{Appendix B}

\section{Score function and Fisher information matrix: uncensored case}

\section{B.1 Penalized score functions}

The penalized log-likelihood function that we are considering takes the form

$$
\begin{aligned}
L_{p}(\boldsymbol{\theta}, \boldsymbol{\alpha}) & =n \log C(\lambda)-n \log (\sigma)+\frac{1}{\lambda} \sum_{i=1}^{n} \epsilon_{i} \\
& -\frac{1}{\lambda^{2}} \sum_{i=1}^{n} e^{\lambda \epsilon_{i}}-\frac{\alpha_{1}}{2} \boldsymbol{\gamma}_{1}^{\top} \mathbf{M}_{1} \boldsymbol{\gamma}_{1}-\cdots-\frac{\alpha_{k}}{2} \boldsymbol{\gamma}_{k}^{\top} \mathbf{M}_{k} \boldsymbol{\gamma}_{k} .
\end{aligned}
$$

By definition, $C(\lambda)=\frac{|\lambda|}{\Gamma\left(\lambda^{-2}\right)}\left(\lambda^{-2}\right)^{\lambda^{-2}}$ and $\epsilon_{i}=\frac{y_{i}-\mathbf{x}_{i}^{\top} \boldsymbol{\beta}-\mathbf{N}_{i 1} \boldsymbol{\gamma}_{1}-\cdots \mathbf{N}_{i k} \boldsymbol{\gamma}_{k}}{\sigma}$, and $\mathbf{x}_{i}$ and $\mathbf{N}_{i l}$ are rows of the matrices $\mathbf{X}$ and $\mathbf{N}_{l}$, respectively, which are defined in chapter 3. If $\lambda \neq 0$, we have that

$$
\begin{aligned}
& \log C(\lambda)=\log (|\lambda|)-\log \Gamma\left(\frac{1}{\lambda^{2}}\right)-2 \frac{\log (|\lambda|)}{\lambda^{2}}, \\
& \frac{\mathrm{d} \log C(\lambda)}{\mathrm{d} \lambda}=\frac{1}{\lambda}+\frac{2}{\lambda^{3}} \psi\left(\frac{1}{\lambda^{2}}\right)-\frac{2}{\lambda^{3}}+4 \frac{\log (|\lambda|)}{\lambda^{3}}
\end{aligned}
$$

and

$$
\frac{\mathrm{d}^{2} \log C(\lambda)}{\mathrm{d} \lambda^{2}}=-\frac{1}{\lambda^{2}}\left[1-\frac{1}{\lambda^{2}}\left\{10-\frac{4}{\lambda^{2}} \psi^{\prime}\left(\frac{1}{\lambda^{2}}\right)-6 \psi\left(\frac{1}{\lambda^{2}}\right)-12 \log (|\lambda|)\right\}\right],
$$


where $\psi(\cdot)$ is the special function digamma, defined by

$$
\psi(y)=[\log (\Gamma(y))]^{\prime} \text { with } y>0
$$

The penalized score function of $\boldsymbol{\theta}$ which is defined by $\mathbf{U}_{p}^{\boldsymbol{\theta}}=\frac{\partial L_{p}(\boldsymbol{\theta}, \boldsymbol{\alpha})}{\partial \boldsymbol{\theta}}$ has the components

$$
\begin{aligned}
U_{p}^{\beta_{j}} & =-\frac{1}{\lambda \sigma} \sum_{i=1}^{n} x_{i j}+\frac{1}{\lambda \sigma} \sum_{i=1}^{n} x_{i j} e^{\lambda \epsilon_{i}}, \text { with } j=1, \cdots, p \\
U_{p}^{\gamma_{j l} l} & =-\frac{1}{\lambda \sigma} \sum_{i=1}^{n} N_{i j l}+\frac{1}{\lambda \sigma} \sum_{i=1}^{n} N_{i j l} e^{\lambda \epsilon_{i}}-\alpha_{l} \sum_{i=1}^{q_{l}} M_{i j l} \gamma_{i l}, \text { with } j=1, \cdots, q_{l} \text { and } l=1, \cdots, k \\
U_{p}^{\sigma} & =-\frac{n}{\sigma}-\frac{1}{\lambda \sigma} \sum_{i=1}^{n} \epsilon_{i}+\frac{1}{\lambda \sigma} \sum_{i=1}^{n} \epsilon_{i} e^{\lambda \epsilon_{i}} \text { and } \\
U_{p}^{\lambda} & =n\left[\frac{1}{\lambda}+\frac{2}{\lambda^{3}}\left\{\psi\left(\frac{1}{\lambda^{2}}\right)+2 \log (|\lambda|)-1\right\}\right]-\frac{1}{\lambda^{2}} \sum_{i=1}^{n} \epsilon_{i}+\frac{2}{\lambda^{3}} \sum_{i=1}^{n} e^{\lambda \epsilon_{i}}-\frac{1}{\lambda^{2}} \sum_{i=1}^{n} \epsilon_{i} e^{\lambda \epsilon_{i}} .
\end{aligned}
$$

In matrix notation,

$$
\begin{aligned}
\mathbf{U}_{p}^{\beta} & =-\frac{1}{\lambda \sigma} \mathbf{X}^{\top}\{(\mathbf{I}-\mathbf{D}(\mathbf{d})\} \mathbf{1}, \\
\mathbf{U}_{p}^{\gamma_{l}} & =-\frac{1}{\lambda \sigma} \mathbf{N}_{l}^{\top}\{\mathbf{I}-\mathbf{D}(\mathbf{d})\} \mathbf{1}-\alpha_{l} \mathbf{M}_{l} \boldsymbol{\gamma}_{l}, \text { with } l=1, \cdots, k \\
U_{p}^{\sigma} & =-\frac{1}{\sigma} \mathbf{1}^{\top} \mathbf{1}-\frac{1}{\lambda \sigma} \mathbf{1}^{\top}\{\mathbf{I}-\mathbf{D}(\mathbf{d})\} \boldsymbol{\epsilon} \text { and } \\
U_{p}^{\lambda} & =n \zeta_{\lambda}-\frac{1}{\lambda^{2}} \mathbf{1}^{\top} \boldsymbol{\epsilon}+\frac{2}{\lambda^{3}} \mathbf{1}^{\top} \mathbf{D}(\mathbf{d}) \mathbf{1}-\frac{1}{\lambda^{2}} \mathbf{1}^{\top} \mathbf{D}(\mathbf{d}) \boldsymbol{\epsilon},
\end{aligned}
$$

where $\zeta_{\lambda}=\frac{1}{\lambda}+\frac{2}{\lambda^{3}}\left\{\psi\left(\frac{1}{\lambda^{2}}\right)+2 \log (|\lambda|)-1\right\}, \mathbf{1}$ is an $(n \times 1)$ vector of ones, $\mathbf{D}(\mathbf{d})$ is a $(n \times n)$ diagonal matrix with elements, $d_{i}=e^{\lambda \epsilon_{i}}$ and $\epsilon_{i}=\frac{y_{i}-\mathbf{x}_{i}^{\top} \boldsymbol{\beta}-\mathbf{N}_{i 1} \gamma_{1}-\cdots \mathbf{N}_{i k} \gamma_{k}}{\sigma}$. 
108APPENDIX B. SCORE FUNCTION AND FISHER INFORMATION MATRIX: UNCENSOI

\section{B.2 Penalized Hessian matrix}

With base in the results of the last subsection we can calculate the second order derivatives for the parameters of interest, which are given by

$$
\begin{aligned}
& \frac{\partial^{2} L_{p}(\boldsymbol{\theta}, \boldsymbol{\alpha})}{\partial \beta_{j} \partial \beta_{j^{\prime}}}=-\frac{1}{\sigma^{2}} \sum_{i=1}^{n} x_{i j} x_{i j^{\prime}} e^{\lambda \epsilon_{i}}, \text { with } j, j^{\prime}=1, \cdots, p \\
& \frac{\partial^{2} L_{p}(\boldsymbol{\theta}, \boldsymbol{\alpha})}{\partial \gamma_{j l} \gamma_{j^{\prime} l}}=-\frac{1}{\sigma^{2}} \sum_{i=1}^{n} N_{i j l} N_{i j^{\prime} l} e^{\lambda \epsilon_{i}}-\alpha_{l} M_{j j^{\prime} l} \text { with } j, j^{\prime}=1, \cdots, q_{l} \text { and } l=1, \cdots, k, \\
& \frac{\partial^{2} L_{p}(\boldsymbol{\theta}, \boldsymbol{\alpha})}{\partial \gamma_{j l} \gamma_{j^{\prime} l^{\prime}}}=-\frac{1}{\sigma^{2}} \sum_{i=1}^{n} N_{i j l} N_{i j^{\prime} l^{\prime}} e^{\lambda \epsilon_{i}} \text { with } j=1, \cdots, q_{l}, j^{\prime}=1, \cdots, q_{l^{\prime}} \text { and } \\
& l, l^{\prime}=1, \cdots, k \text {, but, } l \neq l^{\prime}, \\
& \frac{\partial^{2} L_{p}(\boldsymbol{\theta}, \boldsymbol{\alpha})}{\partial \gamma_{j^{\prime} l} \partial \beta_{j}}=-\frac{1}{\sigma^{2}} \sum_{i=1}^{n} x_{i j} N_{i j^{\prime} l} e^{\lambda \epsilon_{i}} \text { with } j=1, \cdots, p, j^{\prime}=1, \cdots, q_{l} \text { and } l=1, \cdots, k \text {, } \\
& \frac{\partial^{2} L_{p}(\boldsymbol{\theta}, \boldsymbol{\alpha})}{\partial \sigma^{2}}=\frac{1}{\sigma^{2}}\left[n+\frac{2}{\lambda} \sum_{i=1}^{n} \epsilon_{i}\left(1-e^{\lambda \epsilon_{i}}\right)-\sum_{i=1}^{n} \epsilon_{i}^{2} e^{\lambda \epsilon_{i}}\right], \\
& \frac{\partial^{2} L_{p}(\boldsymbol{\theta}, \boldsymbol{\alpha})}{\partial \lambda^{2}}=-\frac{n}{\lambda^{2}} \tau_{\lambda}+\frac{2}{\lambda^{3}} \sum_{i=1}^{n} \epsilon_{i}-\frac{6}{\lambda^{4}} \sum_{i=1}^{n} e^{\lambda \epsilon_{i}}+\frac{4}{\lambda^{3}} \sum_{i=1}^{n} \epsilon_{i} e^{\lambda \epsilon_{i}}-\frac{1}{\lambda^{2}} \sum_{i=1}^{n} \epsilon_{i}^{2} e^{\lambda \epsilon_{i}}, \\
& \frac{\partial^{2} L_{p}(\boldsymbol{\theta}, \boldsymbol{\alpha})}{\partial \gamma_{j l} \partial \sigma}=\frac{1}{\lambda \sigma^{2}}\left[\sum_{i=1}^{n} N_{i j l}-\sum_{i=1}^{n} N_{i j l} e^{\lambda \epsilon_{i}}-\lambda \sum_{i=1}^{n} N_{i j l} \epsilon_{i} e^{\lambda \epsilon_{i}}\right] \text { with } j=1, \cdots, q_{l} \text { and } l=1, \cdots, k \text {, } \\
& \frac{\partial^{2} L_{p}(\boldsymbol{\theta}, \boldsymbol{\alpha})}{\partial \beta_{j} \partial \sigma}=\frac{1}{\lambda \sigma^{2}}\left[\sum_{i=1}^{n} x_{i j}-\sum_{i=1}^{n} x_{i j} e^{\lambda \epsilon_{i}}-\lambda \sum_{i=1}^{n} x_{i j} \epsilon_{i} e^{\lambda \epsilon_{i}}\right] \text {, } \\
& \frac{\partial^{2} L_{p}(\boldsymbol{\theta}, \boldsymbol{\alpha})}{\partial \lambda \partial \beta_{j}}=\frac{1}{\lambda^{2} \sigma}\left[\sum_{i=1}^{n} x_{i j}-\sum_{i=1}^{n} x_{i j} e^{\lambda \epsilon_{i}}+\lambda \sum_{i=1}^{n} x_{i j} \epsilon_{i} e^{\lambda \epsilon_{i}}\right] \text {, } \\
& \frac{\partial^{2} L_{p}(\boldsymbol{\theta}, \boldsymbol{\alpha})}{\partial \lambda \partial \gamma_{j l}}=\frac{1}{\lambda^{2} \sigma}\left[\sum_{i=1}^{n} N_{i j l}-\sum_{i=1}^{n} N_{i j l} e^{\lambda \epsilon_{i}}+\lambda \sum_{i=1}^{n} N_{i j l} \epsilon_{i} e^{\lambda \epsilon_{i}}\right] \text { with } j=1, \cdots, q_{l} \text { and } l=1, \cdots, k \text {, } \\
& \frac{\partial^{2} L_{p}(\boldsymbol{\theta}, \boldsymbol{\alpha})}{\partial \lambda \partial \sigma}=\frac{1}{\lambda^{2} \sigma} \sum_{i=1}^{n} \epsilon_{i}-\frac{1}{\lambda^{2} \sigma} \sum_{i=1}^{n} \epsilon_{i} e^{\lambda \epsilon_{i}}+\frac{1}{\lambda \sigma} \sum_{i=1}^{n} \epsilon_{i}^{2} e^{\lambda \epsilon_{i}} \text {. }
\end{aligned}
$$


where $\tau_{\lambda}=1-\frac{1}{\lambda^{2}}\left\{10-12 \log (|\lambda|)-6 \psi\left(\frac{1}{\lambda^{2}}\right)-\frac{4}{\lambda^{2}} \psi^{\prime}\left(\frac{1}{\lambda^{2}}\right)\right\}$, In matrix notation,

$$
\begin{aligned}
& \frac{\partial^{2} L_{p}(\boldsymbol{\theta}, \boldsymbol{\alpha})}{\partial \boldsymbol{\beta} \partial \boldsymbol{\beta}^{t}}=-\frac{1}{\sigma^{2}} \mathbf{X}^{\top} \mathbf{D}(\mathbf{d}) \mathbf{X}, \\
& \frac{\partial^{2} L_{p}(\boldsymbol{\theta}, \boldsymbol{\alpha})}{\partial \boldsymbol{\gamma}_{l} \partial \boldsymbol{\gamma}_{l^{\top}}^{t}}=-\frac{1}{\sigma^{2}} \mathbf{N}_{l}^{\top} \mathbf{D}(\mathbf{d}) \mathbf{N}_{l}-\alpha \mathbf{M}_{l}, \\
& \frac{\partial^{2} L_{p}(\boldsymbol{\theta}, \boldsymbol{\alpha})}{\partial \sigma^{2}}=\frac{1}{\sigma^{2}}\left[\mathbf{1}^{\top} \mathbf{1}+\frac{2}{\lambda} \mathbf{1}^{\top}\{\mathbf{I}-\mathbf{D}(\mathbf{d})\} \boldsymbol{\epsilon}-\boldsymbol{\epsilon}^{\top} \mathbf{D}(\mathbf{d}) \boldsymbol{\epsilon}\right], \\
& \frac{\partial^{2} L_{p}(\boldsymbol{\theta}, \boldsymbol{\alpha})}{\partial \lambda^{2}}=-\frac{n}{\lambda^{2}} \tau_{\lambda}+\frac{2}{\lambda^{3}} \mathbf{1}^{\top} \boldsymbol{\epsilon}-\frac{6}{\lambda^{4}} \mathbf{1}^{\top} \mathbf{D}(\mathbf{d}) \mathbf{1}+\frac{4}{\lambda^{3}} \mathbf{1}^{\top} \mathbf{D}(\mathbf{d}) \boldsymbol{\epsilon}-\frac{1}{\lambda^{2}} \boldsymbol{\epsilon}^{\top} \mathbf{D}(\mathbf{d}) \boldsymbol{\epsilon}, \\
& \frac{\partial^{2} L_{p}(\boldsymbol{\theta}, \boldsymbol{\alpha})}{\partial \boldsymbol{\gamma}_{l} \partial \boldsymbol{\beta}}=-\frac{1}{\sigma^{2}} \mathbf{N}_{l}^{\top} \mathbf{D}(\mathbf{d}) \mathbf{X}, \text { with } l=1, \cdots, k, \\
& \frac{\partial^{2} L_{p}(\boldsymbol{\theta}, \boldsymbol{\alpha})}{\partial \boldsymbol{\gamma}_{l} \partial \sigma}=\frac{1}{\lambda \sigma^{2}}\left[\mathbf{N}_{l}^{\top} \mathbf{1}-\mathbf{N}_{l}^{\top} \mathbf{D}(\mathbf{d}) \mathbf{1}-\lambda \mathbf{N}_{l}^{\top} \mathbf{D}(\mathbf{d}) \boldsymbol{\epsilon}\right] \text { with } l=1, \cdots, k, \\
& \frac{\partial^{2} L_{p}(\boldsymbol{\theta}, \boldsymbol{\alpha})}{\partial \boldsymbol{\beta} \partial \sigma}=\frac{1}{\lambda \sigma^{2}}\left[\mathbf{1}^{\top} \mathbf{X}-\mathbf{1}^{\top} \mathbf{D}(\mathbf{d}) \mathbf{X}-\lambda \mathbf{X}^{\top} \mathbf{D}(\mathbf{d}) \boldsymbol{\epsilon}\right], \\
& \frac{\partial^{2} L_{p}(\boldsymbol{\theta}, \boldsymbol{\alpha})}{\partial \lambda \partial \boldsymbol{\beta}}=\frac{1}{\lambda^{2} \sigma}\left[\mathbf{1}^{\top} \mathbf{X}-\mathbf{1}^{\top} \mathbf{D}(\mathbf{d}) \mathbf{X}+\lambda \mathbf{X}^{\top} \mathbf{D}(\mathbf{d}) \boldsymbol{\epsilon}\right], \\
& \frac{\partial^{2} L_{p}(\boldsymbol{\theta}, \boldsymbol{\alpha})}{\partial \lambda \partial \boldsymbol{\gamma}_{l}}=\frac{1}{\lambda^{2} \sigma}\left[\mathbf{N}_{l}^{\top} \mathbf{1}-\mathbf{N}_{l}^{\top} \mathbf{D}(\mathbf{d}) \mathbf{1}+\lambda \mathbf{N}_{l}^{\top} \mathbf{D}(\mathbf{d}) \boldsymbol{\epsilon}\right] \text { with } l=1, \cdots, k, \\
& \frac{\partial^{2} L_{p}(\boldsymbol{\theta}, \boldsymbol{\alpha})}{\partial \lambda \partial \sigma}=\frac{1}{\lambda^{2} \sigma}\left[\mathbf{1}^{\top} \boldsymbol{\epsilon}-\mathbf{1}^{\top} \mathbf{D}(\mathbf{d}) \boldsymbol{\epsilon}+\lambda \boldsymbol{\epsilon}^{\top} \mathbf{D}(\mathbf{d}) \boldsymbol{\epsilon}\right] .
\end{aligned}
$$

\section{B.3 Penalized Fisher Information}

For this calculation we will use the regularity conditions

$$
\begin{aligned}
E\left[\frac{\partial L(\boldsymbol{\theta})}{\partial \boldsymbol{\theta}}\right] & =0 \text { and } \\
E\left[\left(\frac{\partial L(\boldsymbol{\theta})}{\partial \boldsymbol{\theta}}\right)\left(\frac{\partial L(\boldsymbol{\theta})}{\partial \boldsymbol{\theta}}\right)^{\top}\right] & =-E\left[\frac{\partial^{2} L(\boldsymbol{\theta})}{\partial \boldsymbol{\theta} \partial \boldsymbol{\theta}^{\top}}\right] .
\end{aligned}
$$


110APPENDIX B. SCORE FUNCTION AND FISHER INFORMATION MATRIX: UNCENSOI

In the case of the vector parameter $\boldsymbol{\beta}$, we obtain

$$
\begin{aligned}
\mathbf{I}_{p}^{\boldsymbol{\beta} \beta} & =-E\left[-\frac{1}{\sigma^{2}} \mathbf{X}^{\top} \mathbf{D}(\mathbf{d}) \mathbf{X}\right] \\
& =\frac{1}{\sigma^{2}} \mathbf{X}^{\top} E[\mathbf{D}(\mathbf{d})] \mathbf{X} \\
& =\frac{r_{\lambda}}{\sigma^{2}} \mathbf{X}^{\top} \mathbf{X},
\end{aligned}
$$

where, $r_{\lambda}=E\left(e^{\lambda \epsilon_{i}}\right)$.

The Fisher information for nonparametric components is

$$
\begin{aligned}
\mathbf{I}_{p}^{\gamma} \gamma_{l} & =-E\left[-\frac{1}{\sigma^{2}} \mathbf{N}_{l}^{\top} \mathbf{D}(\mathbf{d}) \mathbf{N}_{l}-\alpha_{l} \mathbf{M}_{l}\right] \\
& =\frac{1}{\sigma^{2}} \mathbf{N}_{l}^{\top} E[\mathbf{D}(\mathbf{d})] \mathbf{N}_{l}+\alpha_{l} \mathbf{M}_{l} \\
& =\frac{r_{\lambda}}{\sigma^{2}} \mathbf{N}_{l}^{\top} \mathbf{N}_{l}+\alpha_{l} \mathbf{M}_{l}
\end{aligned}
$$

with $l=1, \cdots, k$. The cross information sub-matrices are

$$
\begin{aligned}
\mathbf{I}_{p}^{\boldsymbol{\beta} \gamma_{l}} & =-E\left[-\frac{1}{\sigma^{2}} \mathbf{N}_{l}^{\top} \mathbf{D}(\mathbf{d}) \mathbf{X}\right] \\
& =\frac{1}{\sigma^{2}} \mathbf{N}_{l}^{\top} E[\mathbf{D}(\mathbf{d})] \mathbf{X} \\
& =\frac{r_{\lambda}}{\sigma^{2}} \mathbf{N}_{l}^{\top} \mathbf{X} .
\end{aligned}
$$

Therefore, $\boldsymbol{\beta}$ and $\gamma_{l}$ are not orthogonal with $l=1, \cdots, k$.

$$
\begin{aligned}
\mathbf{I}_{p}^{\gamma_{l} \gamma_{l^{\prime}}} & =-E\left[-\frac{1}{\sigma^{2}} \mathbf{N}_{l}^{\top} \mathbf{D}(\mathbf{d}) \mathbf{N}_{l^{\prime}}\right] \\
& =\frac{1}{\sigma^{2}} \mathbf{N}_{l}^{\top} E[\mathbf{D}(\mathbf{d})] \mathbf{N}_{l^{\prime}} \\
& =\frac{r_{\lambda}}{\sigma^{2}} \mathbf{N}_{l}^{\top} \mathbf{N}_{l^{\prime}}
\end{aligned}
$$


with $l, l^{\prime}=1, \cdots, k$ but $l \neq l^{\prime}$. From $E\left[-\frac{\partial^{2} L(\theta)}{\partial \sigma^{2}}\right]$, we obtain

$$
\begin{aligned}
\mathbf{I}_{p}^{\sigma \sigma} & =-E\left[\frac{1}{\sigma^{2}}\left(\mathbf{1}^{\top} \mathbf{1}+\frac{2}{\lambda} \mathbf{1}^{\top}(\mathbf{I}-\mathbf{D}(\mathbf{d})) \boldsymbol{\epsilon}-\boldsymbol{\epsilon}^{\top} \mathbf{D}(\mathbf{d}) \boldsymbol{\epsilon}\right)\right] \\
& =-\left(\frac{1}{\sigma^{2}} \mathbf{1}^{\top} \mathbf{1}+\frac{2}{\lambda} \mathbf{1}^{\top} E[(\mathbf{I}-\mathbf{D}(\mathbf{d})) \boldsymbol{\epsilon}]-E\left[\boldsymbol{\epsilon}^{\top} \mathbf{D}(\mathbf{d}) \boldsymbol{\epsilon}\right]\right) \\
& =-\left(\frac{1}{\sigma^{2}} \mathbf{1}^{\top} \mathbf{1}+\frac{2}{\lambda} \mathbf{1}^{\top}(E[\boldsymbol{\epsilon}]-E[\mathbf{D}(\mathbf{d}) \boldsymbol{\epsilon}])-E\left[\boldsymbol{\epsilon}^{\top} \mathbf{D}(\mathbf{d}) \boldsymbol{\epsilon}\right]\right) \\
& =-\frac{n}{\sigma^{2}}\left(1+\frac{2}{\lambda}\left(k_{\lambda}-u_{\lambda}\right)-v_{\lambda}\right),
\end{aligned}
$$

with $v_{\lambda}=E\left(\epsilon_{i}^{2} e^{\lambda \epsilon_{i}}\right), k_{\lambda}=E\left(\epsilon_{i}\right)$ and $u_{\lambda}=E\left(\epsilon_{i} e^{\lambda \epsilon_{i}}\right)$.

Remark. In this point is not quite obvious that the last expression to be positive, but in a few lines more the doubt will disappear.

From the condition $E\left[\frac{\partial L(\theta)}{\partial \sigma}\right]=0$, we obtain that

$$
\begin{aligned}
E\left[-\frac{1}{\sigma} \mathbf{1}^{\top} \mathbf{1}-\frac{1}{\lambda \sigma} \mathbf{1}^{\top}(\mathbf{I}-\mathbf{D}(\boldsymbol{d})) \boldsymbol{\epsilon}\right] & =0 \\
-\mathbf{1}^{\top} \mathbf{1}-\frac{1}{\lambda} \mathbf{1}^{\top} E[(\mathbf{I}-\mathbf{D}(\boldsymbol{d})) \boldsymbol{\epsilon}] & =0 \\
-n-\frac{1}{\lambda} \mathbf{1}^{\top}(E[\boldsymbol{\epsilon}]-E[\mathbf{D}(\boldsymbol{d}) \boldsymbol{\epsilon}]) & =0 \\
-n-\frac{1}{\lambda} \mathbf{1}^{\top}\left(k_{\lambda} \mathbf{1}-u_{\lambda} \mathbf{1}\right] & =0 \\
-n-\frac{n}{\lambda}\left(k_{\lambda}-u_{\lambda}\right) & =0 \\
k_{\lambda}-u_{\lambda} & =-\lambda .
\end{aligned}
$$

Therefore,

$$
\mathbf{I}_{p}^{\sigma \sigma}=\frac{n}{\sigma^{2}}\left(1+v_{\lambda}\right)
$$

and the promise is achieved. 
For $\mathbf{I}_{p}^{\boldsymbol{\beta} \sigma}=E\left[-\frac{\partial^{2} L_{p}(\theta)}{\partial \boldsymbol{\beta} \partial \sigma}\right]$, we get

$$
\begin{aligned}
\mathbf{I}_{p}^{\boldsymbol{\beta} \sigma} & =-E\left[\frac{1}{\lambda \sigma^{2}}\left(\mathbf{1}^{\top} \mathbf{X}-\mathbf{1}^{\top} \mathbf{D}(\boldsymbol{d}) \mathbf{X}-\lambda \boldsymbol{\epsilon}^{\top} \mathbf{D}(\boldsymbol{d}) \mathbf{X}\right)\right] \\
& =-\frac{1}{\lambda \sigma^{2}}\left(\mathbf{1}^{\top} \mathbf{X}-\mathbf{1}^{\top} E[\mathbf{D}(\boldsymbol{d})] \mathbf{X}-\lambda E\left[\boldsymbol{\epsilon}^{\top} \mathbf{D}(\boldsymbol{d})\right] \mathbf{X}\right) \\
& =-\frac{1}{\lambda \sigma^{2}}\left(\mathbf{1}^{\top} \mathbf{X}-r_{\lambda} \mathbf{1}^{\top} \mathbf{X}-\lambda u_{\lambda} \mathbf{1}^{\top} \mathbf{X}\right) \\
& =\frac{1}{\lambda \sigma^{2}}\left(r_{\lambda}+\lambda u_{\lambda}-1\right) \mathbf{1}^{\top} \mathbf{X} .
\end{aligned}
$$

In the case of $\mathbf{I}_{p}^{\gamma_{l} \sigma}$, we obtain that

$$
\begin{aligned}
\mathbf{I}_{p}^{\gamma_{l} \sigma} & =-E\left[\frac{1}{\lambda \sigma^{2}}\left(\mathbf{N}_{l}^{\top} \mathbf{1}-\mathbf{N}_{l}^{\top} \mathbf{D}(\boldsymbol{d}) \mathbf{1}-\lambda \mathbf{N}_{l}^{\top} \mathbf{D}(\boldsymbol{d}) \boldsymbol{\epsilon}\right)\right] \\
& =\frac{-1}{\lambda \sigma^{2}}\left(\mathbf{N}_{l}^{\top} \mathbf{1}-\mathbf{N}_{l}^{\top} E[\mathbf{D}(\boldsymbol{d})] \mathbf{1}-\lambda \mathbf{N}_{l}^{\top} E[\mathbf{D}(\boldsymbol{d}) \boldsymbol{\epsilon}]\right) \\
& =\frac{1}{\lambda \sigma^{2}}\left(r_{\lambda}+\lambda u_{\lambda}-1\right) \mathbf{N}_{l}^{\top} \mathbf{1} .
\end{aligned}
$$

Hence, $\boldsymbol{\beta}$ and $\boldsymbol{\gamma}_{l}$ are not orthogonal with $\sigma$.

The next parameter is $\lambda$

$$
\begin{aligned}
\mathbf{I}_{p}^{\lambda \lambda} & =-E\left[-\frac{n}{\lambda^{2}} \tau_{\lambda}+\frac{2}{\lambda^{3}} \mathbf{1}^{\top} \boldsymbol{\epsilon}-\frac{6}{\lambda^{4}} \mathbf{1}^{\top} \mathbf{D}(\boldsymbol{d}) \mathbf{1}+\frac{4}{\lambda^{3}} \mathbf{1}^{\top} \mathbf{D}(\boldsymbol{d}) \boldsymbol{\epsilon}-\frac{1}{\lambda^{2}} \boldsymbol{\epsilon}^{\top} \mathbf{D}(\boldsymbol{d}) \boldsymbol{\epsilon}\right] \\
& =\frac{n}{\lambda^{2}} \tau_{\lambda}-\frac{2}{\lambda^{3}} E\left[\mathbf{1}^{\top} \boldsymbol{\epsilon}\right]+\frac{6}{\lambda^{4}} E\left[\mathbf{1}^{\top} \mathbf{D}(\boldsymbol{d}) \mathbf{1}\right]-\frac{4}{\lambda^{3}} E\left[\mathbf{1}^{\top} \mathbf{D}(\boldsymbol{d}) \boldsymbol{\epsilon}\right]+\frac{1}{\lambda^{2}} E\left[\boldsymbol{\epsilon}^{\top} \mathbf{D}(\boldsymbol{d}) \boldsymbol{\epsilon}\right] \\
= & \frac{n}{\lambda^{2}}\left[\tau_{\lambda}-\frac{2}{\lambda^{2}}\left(\psi\left(\frac{1}{\lambda^{2}}\right)-\log \left(\frac{1}{\lambda^{2}}\right)\right)+\frac{6}{\lambda^{2}} r_{\lambda}-\frac{4}{\lambda} u_{\lambda}+v_{\lambda}\right] .
\end{aligned}
$$

The following one is $\mathbf{I}_{p}^{\lambda \boldsymbol{\beta}}$

$$
\begin{aligned}
\mathbf{I}_{p}^{\lambda \boldsymbol{\beta}} & =-E\left[\frac{1}{\lambda^{2} \sigma}\left(\mathbf{1}^{\top} \mathbf{X}-\mathbf{1}^{\top} \boldsymbol{D}(\boldsymbol{d}) \mathbf{X}+\lambda \mathbf{X}^{\top} \boldsymbol{D}(\boldsymbol{d}) \boldsymbol{\epsilon}\right)\right] \\
& =\frac{1}{\lambda^{2} \sigma}\left(-E\left[\mathbf{1}^{\top} \mathbf{X}\right]+E\left[\mathbf{1}^{\top} \boldsymbol{D}(\boldsymbol{d}) \mathbf{X}\right]-\lambda E\left[\mathbf{X}^{\top} \boldsymbol{D}(\boldsymbol{d}) \boldsymbol{\epsilon}\right]\right) \\
& =\frac{1}{\lambda^{2} \sigma}\left(r_{\lambda}-\lambda u_{\lambda}-1\right) \mathbf{1}^{\top} \mathbf{X} .
\end{aligned}
$$


The next one is $\mathbf{I}_{p}^{\lambda \gamma_{l}}$

$$
\begin{aligned}
\mathbf{I}_{p}^{\lambda \gamma_{l}} & =-E\left[\frac{1}{\lambda^{2} \sigma}\left(\mathbf{N}_{l}^{\top} \mathbf{1}-\mathbf{N}_{l}^{\top} \boldsymbol{D}(\mathbf{d}) \mathbf{1}+\lambda \mathbf{N}_{l}^{\top} \boldsymbol{D}(\mathbf{d}) \epsilon\right)\right] \\
& =\frac{1}{\lambda^{2} \sigma}\left[-E\left[\mathbf{N}_{l}^{\top} \mathbf{1}\right]+E\left[\mathbf{N}_{l}^{\top} \boldsymbol{D}(\boldsymbol{d}) \mathbf{1}\right]-\lambda E\left[\mathbf{N}_{l}^{\top} \boldsymbol{D}(\boldsymbol{d}) \boldsymbol{\epsilon}\right]\right] \\
& =\frac{1}{\lambda^{2} \sigma}\left(r_{\lambda}-\lambda u_{\lambda}-1\right) \mathbf{N}_{l}^{\top} \mathbf{1} .
\end{aligned}
$$

Finally, $\mathbf{I}_{p}^{\lambda \sigma}$

$$
\begin{aligned}
\mathbf{I}_{p}^{\lambda \sigma} & =-E\left[\frac{1}{\lambda^{2} \sigma} \mathbf{1}^{\top} \boldsymbol{\epsilon}-\frac{1}{\lambda^{2} \sigma} \mathbf{1}^{\top} \boldsymbol{D}(\boldsymbol{d}) \boldsymbol{\epsilon}+\frac{1}{\lambda \sigma} \boldsymbol{\epsilon}^{\top} \boldsymbol{D}(\boldsymbol{d}) \boldsymbol{\epsilon}\right] \\
& =-\frac{1}{\lambda^{2} \sigma} E\left[\mathbf{1}^{\top} \boldsymbol{\epsilon}\right]+\frac{1}{\lambda^{2} \sigma} E\left[\mathbf{1}^{\top} \boldsymbol{D}(\boldsymbol{d}) \boldsymbol{\epsilon}\right]-\frac{1}{\lambda \sigma} E\left[\boldsymbol{\epsilon}^{\top} \boldsymbol{D}(\boldsymbol{d}) \boldsymbol{\epsilon}\right] \\
& =\frac{n}{\lambda^{2} \sigma}\left[-\frac{\psi\left(\frac{1}{\lambda^{2}}\right)-\log \left(\frac{1}{\lambda^{2}}\right)}{\lambda}+u_{\lambda}-\lambda v_{\lambda}\right] .
\end{aligned}
$$

Hence, $\boldsymbol{\beta}, \gamma_{l}$ and $\sigma$ are not orthogonal with $\lambda$.

The penalized Fisher information matrix takes the form

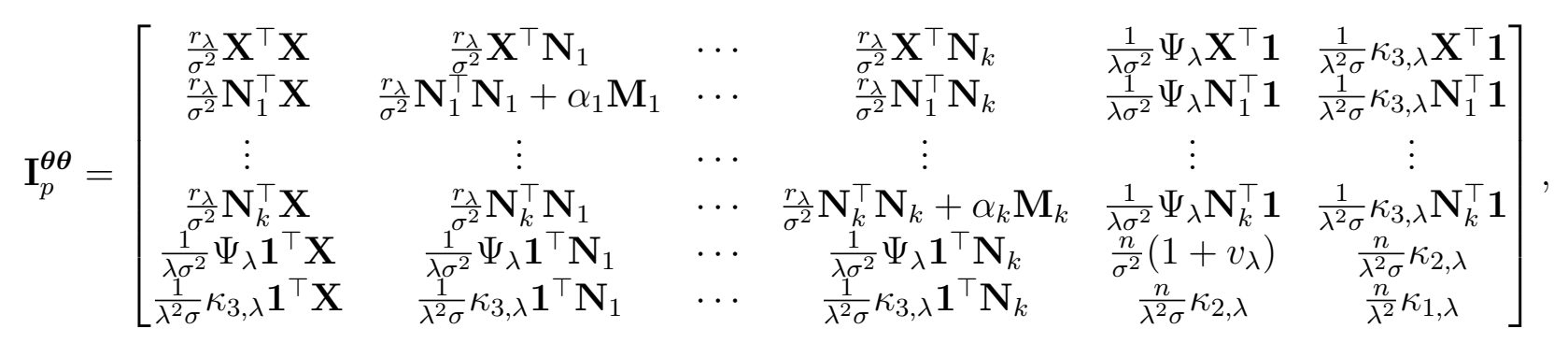

where $\boldsymbol{\theta}=\left(\boldsymbol{\beta}^{\top}, \boldsymbol{\gamma}_{1}^{\top}, \cdots, \boldsymbol{\gamma}_{k}^{\top}, \sigma, \lambda\right)^{\top}, \Psi_{\lambda}=r_{\lambda}+\lambda u_{\lambda}-1$ and

$$
\begin{aligned}
& \kappa_{1, \lambda}=\tau_{\lambda}-\frac{2}{\lambda^{2}}\left(\psi\left(\frac{1}{\lambda^{2}}\right)-\log \left(\frac{1}{\lambda^{2}}\right)\right)+\frac{6}{\lambda^{2}} r_{\lambda}-\frac{4}{\lambda} u_{\lambda}+v_{\lambda}, \\
& \kappa_{2, \lambda}=u_{\lambda}-\frac{\psi\left(\frac{1}{\lambda^{2}}\right)-\log \left(\frac{1}{\lambda^{2}}\right)}{\lambda}-\lambda v_{\lambda} \text { and } \\
& \kappa_{3, \lambda}=r_{\lambda}-\lambda u_{\lambda}-1 .
\end{aligned}
$$


114APPENDIX B. SCORE FUNCTION AND FISHER INFORMATION MATRIX: UNCENSOI

\section{B.4 The case $\lambda=1$}

When $\lambda=1, \epsilon_{i} \sim \operatorname{GLG}(0,1,1)$ hence $e^{\epsilon_{i}} \sim \operatorname{Weibull}(1,1)$

or equivalently $e^{\epsilon_{i}} \sim \operatorname{Exp}(1)$. Therefore,

$$
\begin{aligned}
& r_{1}=\Gamma(1)=1, \\
& k_{1}=E\left(\epsilon e^{\epsilon}\right)=1-\gamma \text { and } \\
& v_{1}=E\left(\epsilon^{2} e^{\epsilon}\right)=\pi^{2}+\gamma^{2}-2 \gamma,
\end{aligned}
$$

according to Paula and Rojas (1997). 


\section{Appendix C}

\section{Some useful derivations for the censored case}

\section{C.1 Penalized likelihood functions}

The penalized log-likelihood function that we are considering have two situations.

When $\lambda>0$,

$$
\begin{aligned}
L_{p}(\boldsymbol{\theta}, \boldsymbol{\alpha}) & =\sum_{i \in D}\left[\log \left(\frac{c(\lambda)}{\sigma}\right)+\frac{1}{\lambda} \epsilon_{i}-\frac{1}{\lambda^{2}} e^{\lambda \epsilon_{i}}\right]+\sum_{i \in C} \log \left(1-\mathrm{I}\left(\lambda^{-2}, \lambda^{-2} e^{\lambda \epsilon_{i}}\right)\right) \\
& -\frac{\alpha_{1}}{2} \sum_{i=1}^{q_{1}} \sum_{j=1}^{q_{1}} M_{i j 1} \gamma_{i 1} \gamma_{j 1}-\cdots-\frac{\alpha_{k}}{2} \sum_{i=1}^{q_{k}} \sum_{j=1}^{q_{k}} M_{i j k} \gamma_{i k} \gamma_{j k} .
\end{aligned}
$$

and when $\lambda<0$,

$$
\begin{aligned}
L_{p}(\boldsymbol{\theta}, \boldsymbol{\alpha}) & =\sum_{i \in D}\left[\log \left(\frac{c(\lambda)}{\sigma}\right)+\frac{1}{\lambda} \epsilon_{i}-\frac{1}{\lambda^{2}} e^{\lambda \epsilon_{i}}\right]+\sum_{i \in C} \log \left(\mathrm{I}\left(\lambda^{-2}, \lambda^{-2} e^{\lambda \epsilon_{i}}\right)\right) \\
& -\frac{\alpha_{1}}{2} \sum_{i=1}^{q_{1}} \sum_{j=1}^{q_{1}} M_{i j 1} \gamma_{i 1} \gamma_{j 1}-\cdots-\frac{\alpha_{k}}{2} \sum_{i=1}^{q_{k}} \sum_{j=1}^{q_{k}} M_{i j k} \gamma_{i k} \gamma_{j k} .
\end{aligned}
$$

where $\epsilon_{i}=\frac{y_{i}-\mathbf{x}_{i}^{\top} \beta-\mathbf{N}_{i 1} \gamma_{1}-\cdots \mathbf{N}_{i k} \gamma_{k}}{\sigma}$ and $\mathrm{I}(\lambda, a)$ is the incomplete gamma function,

$$
\mathrm{I}(\lambda, a)=\frac{1}{\Gamma(\lambda)} \int_{0}^{a} x^{\lambda-1} e^{-x} d x
$$




\section{C.2 Penalized score functions}

The new element in the penalized log-likelihood function with respect to the analogy in the previous appendix is

$$
\sum_{i \in C} \log \left(1-\mathrm{I}\left(\lambda^{-2}, \lambda^{-2} e^{\lambda \epsilon_{i}}\right)\right) .
$$

We will derive this quantity in some particular situations.

Let $a_{i}=\lambda^{-2} e^{\lambda \epsilon_{i}}$, for $i=1, \cdots, n$ and $S\left(a_{i}, \lambda\right)=1-\mathrm{I}\left(\lambda^{-2}, a_{i}\right)$. Then,

$$
\begin{aligned}
\frac{\partial \log S\left(a_{i}, \lambda\right)}{\partial \beta_{j}} & =\frac{\partial \log S\left(a_{i}, \lambda\right)}{\partial a_{i}} \frac{\partial a_{i}}{\partial \beta_{j}} \\
\frac{\partial a_{i}}{\partial \beta_{j}} & =\frac{\partial a_{i}}{\partial \epsilon_{i}} \frac{\partial \epsilon_{i}}{\partial \beta_{j}} .
\end{aligned}
$$

Since

$$
\begin{aligned}
\frac{\partial \epsilon_{i}}{\partial \beta_{j}} & =\frac{-x_{i j}}{\sigma} \\
\frac{\partial a_{i}}{\partial \epsilon_{i}} & =\frac{1}{\lambda} e^{\lambda \epsilon_{i}} \\
\frac{\partial a_{i}}{\partial \beta_{j}} & =\frac{-x_{i j}}{\lambda \sigma} e^{\lambda \epsilon_{i}} \text { and } \\
\frac{\partial S\left(a_{i}, \lambda\right)}{\partial a_{i}} & =\frac{-a_{i}^{\lambda^{-2}-1}}{\Gamma\left(\lambda^{-2}\right)} e^{-a_{i}}
\end{aligned}
$$

Hence,

$$
\frac{\partial \log S\left(a_{i}, \lambda\right)}{\partial \beta_{j}}=\frac{\lambda x_{i j}}{\sigma \Gamma\left(\lambda^{-2}\right)} \frac{a_{i}^{\lambda^{-2}}}{S\left(a_{i}, \lambda\right)} e^{-a_{i}}
$$

for $j=1, \ldots, p$.

In the case of the penalized score function with respect to $\sigma$, we have

$$
\begin{aligned}
\frac{\partial \log S\left(a_{i}, \lambda\right)}{\partial \sigma} & =\frac{\partial \log S\left(a_{i}, \lambda\right)}{\partial a_{i}} \frac{\partial a_{i}}{\partial \sigma} \\
\frac{\partial a_{i}}{\partial \sigma} & =\frac{\partial a_{i}}{\partial \epsilon_{i}} \frac{\partial \epsilon_{i}}{\partial \sigma} .
\end{aligned}
$$


Since

$$
\begin{aligned}
\frac{\partial \epsilon_{i}}{\partial \sigma} & =\frac{-\epsilon_{i}}{\sigma} \\
\frac{\partial a_{i}}{\partial \epsilon_{i}} & =\frac{1}{\lambda} e^{\lambda \epsilon_{i}} \\
\frac{\partial a_{i}}{\partial \sigma} & =\frac{-\lambda \epsilon_{i} a_{i}}{\sigma} \quad \text { and } \\
\frac{\partial S\left(a_{i}, \lambda\right)}{\partial a_{i}} & =\frac{-a_{i}^{\lambda^{-2}-1}}{\Gamma\left(\lambda^{-2}\right)} e^{-a_{i}}
\end{aligned}
$$

Hence,

$$
\frac{\partial \log S\left(a_{i}, \lambda\right)}{\partial \sigma}=\frac{\lambda}{\sigma \Gamma\left(\lambda^{-2}\right)} \frac{\epsilon_{i} a_{i}^{\lambda^{-2}}}{S\left(a_{i}, \lambda\right)} e^{-a_{i}}
$$

In the case of the penalized score function with respect to $\lambda$, we have

$$
\begin{aligned}
\frac{\partial a_{i}}{\partial \lambda} & =a_{i}\left(\epsilon_{i}-2 \lambda^{-1}\right) \\
\frac{\partial I\left(\lambda^{-2}, a_{i}\right)}{\partial \lambda} & =2 \lambda^{-3} \psi\left(\lambda^{-2}\right) I\left(\lambda^{-2}, a_{i}\right)+\frac{1}{\Gamma\left(\lambda^{-2}\right)} a_{i}^{\lambda^{-2}} e^{-a_{i}}\left(\epsilon_{i}-2 \lambda^{-1}\right)
\end{aligned}
$$

SO,

$$
\frac{\partial \log S\left(a_{i}, \lambda\right)}{\partial \lambda}=-\frac{2 \lambda^{-3} \psi\left(\lambda^{-2}\right) \mathrm{I}\left(\lambda^{-2}, a_{i}\right)+\frac{1}{\Gamma\left(\lambda^{-2}\right)} a_{i}^{\lambda^{-2}} e^{-a_{i}}\left(\epsilon_{i}-2 \lambda^{-1}\right)}{S\left(a_{i}, \lambda\right)}
$$

\section{C.3 Penalized observed information matrix}

First, we obtain

$$
\frac{\partial^{2} \log S\left(a_{i}, \lambda\right)}{\partial \beta_{l} \partial \beta_{j}}=\frac{\lambda x_{i j}}{\sigma \Gamma\left(\lambda^{-2}\right)} \frac{\partial}{\partial \beta_{l}}\left[\frac{a_{i}^{\lambda^{-2}}}{S\left(a_{i}, \lambda\right)} e^{-a_{i}}\right]
$$

and

$$
\frac{\partial}{\partial \beta_{l}}\left[\frac{a_{i}^{\lambda^{-2}}}{S\left(a_{i}, \lambda\right)} e^{-a_{i}}\right]=\frac{\frac{\partial}{\partial \beta_{l}}\left[a_{i}^{\lambda^{-2}} e^{-a_{i}}\right] S\left(a_{i}, \lambda\right)-a_{i}^{\lambda^{-2}} e^{-a_{i}} \frac{\partial}{\partial \beta_{l}} S\left(a_{i}, \lambda\right)}{\left[S\left(a_{i}, \lambda\right)\right]^{2}} .
$$


But

$$
\begin{aligned}
\frac{\partial}{\partial \beta_{l}}\left[a_{i}^{\lambda^{-2}} e^{-a_{i}}\right] & =\frac{x_{i l}}{\lambda \sigma} a_{i}^{\lambda^{-2}} e^{-a_{i}}\left(a_{i}-\frac{1}{\lambda^{2}}\right) \\
a_{i}^{\lambda^{-2}} e^{-a_{i}} \frac{\partial}{\partial \beta_{l}} S\left(a_{i}, \lambda\right) & =\frac{\lambda x_{i l}}{\sigma \Gamma\left(\lambda^{-2}\right)}\left(a_{i}^{\lambda^{-2}} e^{-a_{i}}\right)^{2} .
\end{aligned}
$$

Then,

$$
\frac{\partial^{2} \log S\left(a_{i}, \lambda\right)}{\partial \beta_{l} \partial \beta_{j}}=\frac{\lambda^{2} x_{i j} x_{i l}}{\sigma^{2} \Gamma\left(\lambda^{-2}\right) S\left(a_{i}, \lambda\right)} a_{i}^{\lambda^{-2}} e^{-a_{i}}\left[a_{i}-\lambda^{-2}-\frac{a_{i}^{\lambda^{-2}} e^{-a_{i}}}{\Gamma\left(\lambda^{-2}\right) S\left(a_{i}, \lambda\right)}\right],
$$

for $l, j=1, \ldots, p$.

In the case of $\sigma$ component of the observed information matrix, we obtain

$$
\frac{\partial^{2} \log S\left(a_{i}, \lambda\right)}{\partial \sigma^{2}}=\frac{\lambda}{\Gamma\left(\lambda^{-2}\right)} \frac{\partial}{\partial \sigma}\left[\frac{\epsilon_{i} a_{i}^{\lambda^{-2}}}{\sigma S\left(a_{i}, \lambda\right)} e^{-a_{i}}\right]
$$

But

$$
\begin{aligned}
\frac{\partial}{\partial \sigma}\left[\frac{\epsilon_{i} a_{i}^{\lambda^{-2}} e^{-a_{i}}}{\sigma S\left(a_{i}, \lambda\right)}\right] & =\frac{\frac{\partial}{\partial \sigma}\left[\epsilon_{i} a_{i}^{\lambda^{-2}} e^{-a_{i}}\right] \sigma S\left(a_{i}, \lambda\right)-\epsilon_{i} a_{i}^{\lambda^{-2}} e^{-a_{i}} \frac{\partial}{\partial \sigma}\left[\sigma S\left(a_{i}, \lambda\right)\right]}{\left[\sigma S\left(a_{i}, \lambda\right)\right]^{2}} \\
\frac{\partial}{\partial \sigma}\left[\epsilon_{i} a_{i}^{\lambda^{-2}} e^{-a_{i}}\right] & =\frac{\partial}{\partial \sigma}\left[\epsilon_{i} a_{i}^{\lambda^{-2}}\right] e^{-a_{i}}+\epsilon_{i} a_{i}^{\lambda^{-2}} \frac{\partial}{\partial \sigma} e^{-a_{i}}, \\
\frac{\partial}{\partial \sigma}\left[\epsilon_{i} a_{i}^{\lambda^{-2}}\right] & =a_{i}^{\lambda^{-2}} \frac{\partial}{\partial \sigma} \epsilon_{i}+\epsilon_{i} \frac{\partial}{\partial \sigma} a_{i}^{\lambda^{-2}} \\
& =-\frac{\epsilon_{i} a_{i}^{\lambda^{-2}}}{\sigma}-\frac{\epsilon_{i}^{2} a_{i}^{\lambda^{-2}}}{\lambda \sigma}
\end{aligned}
$$

and

$$
\frac{\partial}{\partial \sigma} e^{-a_{i}}=\frac{\lambda \epsilon_{i} a_{i} e^{-a_{i}}}{\sigma}
$$

Then,

$$
\frac{\partial}{\partial \sigma}\left[\epsilon_{i} a_{i}^{\lambda^{-2}} e^{-a_{i}}\right]=\frac{\epsilon_{i} a_{i}^{\lambda^{-2}} e^{-a_{i}}}{\sigma}\left[\lambda \epsilon_{i} a_{i}-1-\frac{\epsilon_{i}}{\lambda}\right]
$$

and

$$
\frac{\partial}{\partial \sigma}\left[\sigma S\left(a_{i}, \lambda\right)\right]=S\left(a_{i}, \lambda\right)+\frac{\lambda \epsilon_{i} a_{i}^{\lambda^{-2}} e^{-a_{i}}}{\Gamma\left(\lambda^{-2}\right)}
$$


Hence,

$$
\frac{\partial^{2} \log S\left(a_{i}, \lambda\right)}{\partial \sigma^{2}}=\frac{\lambda^{2} \epsilon_{i}^{2} a_{i}^{\lambda^{-2}} e^{-a_{i}}}{\sigma^{2} \Gamma\left(\lambda^{-2}\right) S\left(a_{i}, \lambda\right)}\left[a_{i}-\frac{2}{\lambda \epsilon_{i}}-\lambda^{-2}-\frac{a_{i}^{\lambda^{-2}} e^{-a_{i}}}{\Gamma\left(\lambda^{-2}\right) S\left(a_{i}, \lambda\right)}\right] .
$$

In the case of $\lambda^{2}$ component of the observed information matrix, we know that

$$
\frac{\partial^{2} \log S\left(a_{i}, \lambda\right)}{\partial \lambda^{2}}=-\frac{\partial^{2} I\left(\lambda^{-2}, a_{i}\right)}{\partial \lambda^{2}} \frac{1}{S\left(a_{i}, \lambda\right)}-\left(\frac{\partial I\left(\lambda^{-2}, a_{i}\right)}{\partial \lambda}\right)^{2} \frac{1}{S\left(a_{i}, \lambda\right)^{2}} .
$$

So, we are concentrating in computing $\frac{\partial^{2} I\left(\lambda^{-2}, a_{i}\right)}{\partial \lambda^{2}}$. We obtain

$$
\begin{aligned}
\frac{\partial}{\partial \lambda}\left[\frac{\partial I\left(\lambda^{-2}, a_{i}\right)}{\partial \lambda}\right]= & \frac{\partial}{\partial \lambda}\left[-2 \lambda^{-3} \psi\left(\lambda^{-2}\right) I\left(\lambda^{-2}, a_{i}\right)\right] \\
& +\frac{\partial}{\partial \lambda}\left[\frac{1}{\Gamma\left(\lambda^{-2}\right)} a_{i}^{\lambda^{-2}} e^{-a_{i}}\left(\epsilon_{i}-2 \lambda^{-1}\right)\right], \\
\frac{\partial}{\partial \lambda}\left[-2 \lambda^{-3} \psi\left(\lambda^{-2}\right) I\left(\lambda^{-2}, a_{i}\right)\right]= & 6 \lambda^{-4} \psi\left(\lambda^{-2}\right) I\left(\lambda^{-2}, a_{i}\right)+4 \lambda^{-6} \psi^{\prime}\left(\lambda^{-2}\right) I\left(\lambda^{-2}, a_{i}\right) \\
& -2 \lambda^{-3} \psi\left(\lambda^{-2}\right)\left[-2 \lambda^{-3} \psi\left(\lambda^{-2}\right) I\left(\lambda^{-2}, a_{i}\right)\right. \\
& \left.+\frac{1}{\Gamma\left(\lambda^{-2}\right)} a_{i}^{\lambda^{-2}} e^{-a_{i}}\left(\epsilon_{i}-2 \lambda^{-1}\right)\right], \\
\frac{\partial}{\partial \lambda} a_{i}^{\lambda^{-2}}= & \lambda^{-2}\left[\epsilon_{i}-2 \lambda^{-1}\left(\log a_{i}+1\right)\right] a_{i}^{\lambda^{-2}}, \\
\frac{\partial}{\partial \lambda} e^{-a_{i}}= & a_{i} e^{-a_{i}}\left(2 \lambda^{-1}-\epsilon_{i}\right) \text { and } \\
\frac{\partial}{\partial \lambda}\left(\epsilon_{i}-2 \lambda^{-1}\right)= & 2 \lambda^{-2} .
\end{aligned}
$$

Combining the previous expressions

$$
\begin{aligned}
\frac{\partial^{2} I\left(\lambda^{-2}, a_{i}\right)}{\partial \lambda^{2}}= & 6 \lambda^{-4} \psi\left(\lambda^{-2}\right) I\left(\lambda^{-2}, a_{i}\right)+4 \lambda^{-6} \psi^{\prime}\left(\lambda^{-2}\right) I\left(\lambda^{-2}, a_{i}\right) \\
& -2 \lambda^{-3} \psi\left(\lambda^{-2}\right)\left[-2 \lambda^{-3} \psi\left(\lambda^{-2}\right) I\left(\lambda^{-2}, a_{i}\right)+\frac{1}{\Gamma\left(\lambda^{-2}\right)} a_{i}^{\lambda^{-2}} e^{-a_{i}}\left(\epsilon_{i}-2 \lambda^{-1}\right)\right] \\
& +a_{i}^{\lambda^{-2}} e^{-a_{i}}\left[-2 \lambda^{-3} \psi\left(\lambda^{-2}\right)\left(\epsilon_{i}-2 \lambda^{-1}\right)\right. \\
& +\frac{\lambda^{-2}}{\Gamma\left(\lambda^{-2}\right)}\left[\epsilon_{i}-2 \lambda^{-1}\left(\log a_{i}+1\right)\right]\left(\epsilon_{i}-2 \lambda^{-1}\right) \\
& \left.+\frac{a_{i}}{\Gamma\left(\lambda^{-2}\right)}\left(2 \lambda^{-1}-\epsilon_{i}\right)+\frac{2 \lambda^{-2}}{\Gamma\left(\lambda^{-2}\right)}\right] .
\end{aligned}
$$


Therefore,

$$
\begin{aligned}
\frac{\partial^{2} \log S\left(a_{i}, \lambda\right)}{\partial \lambda^{2}} & =-\left[-3 \lambda^{-4} \psi\left(\lambda^{-2}\right) I\left(\lambda^{-2}, a_{i}\right)-2 \lambda^{-6} \psi^{\prime}\left(\lambda^{-2}\right) I\left(\lambda^{-2}, a_{i}\right)\right. \\
& +\lambda^{-3} \psi\left(\lambda^{-2}\right)\left[\lambda^{-3} \psi\left(\lambda^{-2}\right) I\left(\lambda^{-2}, a_{i}\right)+\frac{1}{\Gamma\left(\lambda^{-2}\right)} a_{i}^{\lambda^{-2}} e^{-a_{i}}\left(\epsilon_{i}-2 \lambda^{-1}\right)\right] \\
& +a_{i}^{\lambda^{-2}} e^{-a_{i}}\left[-2 \lambda^{-3} \psi\left(\lambda^{-2}\right)\left(\epsilon_{i}-2 \lambda^{-1}\right)+\frac{\lambda^{-2}}{\Gamma\left(\lambda^{-2}\right)}\left[\epsilon_{i}-2 \lambda^{-1}\left(\log a_{i}+1\right)\right]\left(\epsilon_{i}-2 \lambda^{-1}\right)\right. \\
& \left.\left.+\frac{a_{i}}{\Gamma\left(\lambda^{-2}\right)}\left(2 \lambda^{-1}-\epsilon_{i}\right)+\frac{2 \lambda^{-2}}{\Gamma\left(\lambda^{-2}\right)}\right]\right] \frac{1}{S\left(a_{i}, \lambda\right)} \\
& -\left[\frac{\lambda^{-3} \psi\left(\lambda^{-2}\right) I\left(\lambda^{-2}, a_{i}\right)+\frac{1}{\Gamma\left(\lambda^{-2}\right)} a_{i}^{\lambda^{-2}} e^{-a_{i}}\left(\epsilon_{i}-2 \lambda^{-1}\right)}{S\left(a_{i}, \lambda\right)}\right]^{2} \frac{1}{S\left(a_{i}, \lambda\right)} .
\end{aligned}
$$

Finally, the mixed partial derivatives are

$$
\begin{aligned}
\frac{\partial}{\partial \lambda}\left[\frac{\partial \log S\left(a_{i}, \lambda\right)}{\partial \beta_{j}}\right] & =\frac{\partial}{\partial \lambda}\left[\frac{\lambda x_{i j}}{\sigma \Gamma\left(\lambda^{-2}\right)} \frac{a_{i}^{\lambda^{-2}}}{S\left(a_{i}, \lambda\right)} e^{-a_{i}}\right] \\
& =\frac{x_{i j}}{\sigma} \frac{\partial}{\partial \lambda}\left[\frac{\lambda}{\Gamma\left(\lambda^{-2}\right)} \frac{a_{i}^{\lambda^{-2}}}{S\left(a_{i}, \lambda\right)} e^{-a_{i}}\right] \\
& =\frac{x_{i j}}{\sigma}\left[\frac{\partial}{\partial \lambda}\left(\lambda a_{i}^{\lambda^{-2}} e^{-a_{i}}\right)\left(\frac{1}{\Gamma\left(\lambda^{-2}\right) S\left(a_{i}, \lambda\right)}\right)+\left(\lambda a_{i}^{\lambda^{-2}} e^{-a_{i}}\right) \frac{\partial}{\partial \lambda}\left(\frac{1}{\Gamma\left(\lambda^{-2}\right) S\left(a_{i}, \lambda\right)}\right)\right] \\
& =\frac{x_{i j}}{\sigma} a_{i}^{\lambda^{-2}} e^{-a_{i}}\left[\frac{1+\lambda^{-1}\left[\epsilon_{i}-2 \lambda^{-1}\left(\log a_{i}+1\right)\right]+\lambda a_{i}\left(2 \lambda^{-1}-\epsilon_{i}\right)}{\Gamma\left(\lambda^{-2}\right) S\left(a_{i}, \lambda\right)}\right. \\
& \left.-\lambda \frac{\Gamma\left(\lambda^{-2}\right) \lambda^{-3} \psi\left(\lambda^{-2}\right)\left(1-2 S\left(a_{i}, \lambda\right)\right)-a_{i}^{\lambda^{-2}} e^{-a_{i}}\left(\epsilon_{i}-2 \lambda^{-1}\right)}{\left(\Gamma\left(\lambda^{-2}\right) S\left(a_{i}, \lambda\right)\right)^{2}}\right]
\end{aligned}
$$

for $j=1, \ldots, p$. 
The next one

$$
\begin{aligned}
\frac{\partial}{\partial \lambda}\left[\frac{\partial \log S\left(a_{i}, \lambda\right)}{\partial \gamma_{j l}}\right] & =\frac{\partial}{\partial \lambda}\left[\frac{\lambda N_{i j l}}{\sigma \Gamma\left(\lambda^{-2}\right)} \frac{a_{i}^{\lambda^{-2}}}{S\left(a_{i}, \lambda\right)} e^{-a_{i}}\right] \\
& =\frac{N_{i j l}}{\sigma} \frac{\partial}{\partial \lambda}\left[\frac{\lambda}{\Gamma\left(\lambda^{-2}\right)} \frac{a_{i}^{\lambda^{-2}}}{S\left(a_{i}, \lambda\right)} e^{-a_{i}}\right] \\
& =\frac{N_{i j l}}{\sigma} a_{i}^{\lambda^{-2}} e^{-a_{i}}\left[\frac{1+\lambda^{-1}\left[\epsilon_{i}-2 \lambda^{-1}\left(\log a_{i}+1\right)\right]+\lambda a_{i}\left(2 \lambda^{-1}-\epsilon_{i}\right)}{\Gamma\left(\lambda^{-2}\right) S\left(a_{i}, \lambda\right)}\right. \\
& \left.-\lambda \frac{\Gamma\left(\lambda^{-2}\right) \lambda^{-3} \psi\left(\lambda^{-2}\right)\left(1-2 S\left(a_{i}, \lambda\right)\right)-a_{i}^{\lambda^{-2}} e^{-a_{i}}\left(\epsilon_{i}-2 \lambda^{-1}\right)}{\left(\Gamma\left(\lambda^{-2}\right) S\left(a_{i}, \lambda\right)\right)^{2}}\right]
\end{aligned}
$$

for $j=1, \ldots, q_{l}$ and $l=1, \ldots, k$, and

$$
\begin{aligned}
\frac{\partial}{\partial \lambda}\left[\frac{\partial \log S\left(a_{i}, \lambda\right)}{\partial \sigma}\right] & =\frac{\partial}{\partial \lambda}\left[\frac{\lambda \epsilon_{i}}{\sigma \Gamma\left(\lambda^{-2}\right)} \frac{a_{i}^{\lambda^{-2}}}{S\left(a_{i}, \lambda\right)} e^{-a_{i}}\right] \\
& =\frac{\epsilon_{i}}{\sigma} \frac{\partial}{\partial \lambda}\left[\frac{\lambda}{\Gamma\left(\lambda^{-2}\right)} \frac{a_{i}^{\lambda^{-2}}}{S\left(a_{i}, \lambda\right)} e^{-a_{i}}\right] \\
& =\frac{\epsilon_{i}}{\sigma} a_{i}^{\lambda^{-2}} e^{-a_{i}}\left[\frac{1+\lambda^{-1}\left[\epsilon_{i}-2 \lambda^{-1}\left(\log a_{i}+1\right)\right]+\lambda a_{i}\left(2 \lambda^{-1}-\epsilon_{i}\right)}{\Gamma\left(\lambda^{-2}\right) S\left(a_{i}, \lambda\right)}\right. \\
& \left.-\lambda \frac{\Gamma\left(\lambda^{-2}\right) \lambda^{-3} \psi\left(\lambda^{-2}\right)\left(1-2 S\left(a_{i}, \lambda\right)\right)-a_{i}^{\lambda^{-2}} e^{-a_{i}}\left(\epsilon_{i}-2 \lambda^{-1}\right)}{\left(\Gamma\left(\lambda^{-2}\right) S\left(a_{i}, \lambda\right)\right)^{2}}\right]
\end{aligned}
$$




\section{Appendix D}

\section{Local influence analysis}

\section{D.1 The perturbation schemes}

In this appendix we discuss two perturbation schemes applied to the models in chapters 3 and 4 .

\section{D.1.1 Case-weight perturbation: uncensored case}

For this perturbation scheme we assume that

$$
L_{p}(\boldsymbol{\theta}, \boldsymbol{\alpha} \mid \boldsymbol{\omega})=\sum_{i} \omega_{i} L_{p_{i}}(\boldsymbol{\theta})-\frac{\alpha_{1}}{2} \boldsymbol{\gamma}_{1}^{\top} \mathbf{M}_{1} \boldsymbol{\gamma}_{1}-\cdots-\frac{\alpha_{k}}{2} \boldsymbol{\gamma}_{k}^{\top} \mathbf{M}_{k} \boldsymbol{\gamma}_{k}
$$

where $0<\omega_{i} \leq 1$ and the reference point is $\boldsymbol{\omega}_{0}=(1, \cdots, 1)^{\top}$. Then, we obtain

$$
\begin{aligned}
\left(\Delta_{\boldsymbol{\beta} \omega}\right)_{i j} & =-\frac{x_{i j}}{\lambda \sigma}+\frac{1}{\lambda \sigma} x_{i j} e^{\lambda \epsilon_{i}}, \quad j=1, \cdots, p \\
\left(\Delta_{\gamma_{l} \omega}\right)_{i j} & =-\frac{1}{\lambda \sigma} N_{i j l}+\frac{1}{\lambda \sigma} N_{i j l} e^{\lambda \epsilon_{i}}, \quad j=1, \cdots, q_{l} \\
\left(\Delta_{\sigma \omega}\right)_{i} & =-\frac{1}{\sigma}-\frac{\epsilon_{i}}{\lambda \sigma}+\frac{1}{\lambda \sigma} \epsilon_{i} e^{\lambda \epsilon_{i}} \text { and } \\
\left(\Delta_{\lambda \omega}\right)_{i} & =\zeta_{\lambda}-\frac{\epsilon_{i}}{\lambda^{2}}+\frac{2}{\lambda^{3}} e^{\lambda \epsilon_{i}}-\frac{1}{\lambda^{2}} \epsilon_{i} e^{\lambda \epsilon_{i}}
\end{aligned}
$$

for $i=1, \cdots, n, l=1, \cdots, k$, and $\zeta_{\lambda}=\frac{1}{\lambda}+\frac{2}{\lambda^{3}}\left(\psi\left(\frac{1}{\lambda^{2}}\right)+2 \log |\lambda|-1\right)$. 


\section{D.1.2 Case-weight perturbation: censored case}

In this case we assume that

$$
L_{p}(\boldsymbol{\theta}, \boldsymbol{\alpha} \mid \boldsymbol{\omega})=\sum_{i} \omega_{i}\left[\left(1-\delta_{i}\right) L_{p_{i}}(\boldsymbol{\theta})+\delta_{i} \log \left(1-F_{\epsilon}\left(\epsilon_{i} ; \boldsymbol{\theta}\right)\right)\right]-\frac{\alpha_{1}}{2} \boldsymbol{\gamma}_{1}^{\top} \mathbf{M}_{1} \boldsymbol{\gamma}_{1}-\cdots-\frac{\alpha_{k}}{2} \boldsymbol{\gamma}_{k}^{\top} \mathbf{M}_{k} \boldsymbol{\gamma}_{k}
$$

where $0<\omega_{i} \leq 1$, the reference point is $\boldsymbol{\omega}_{0}=(1, \cdots, 1)^{\top}$ and $F_{\epsilon}\left(\epsilon_{i} ; \boldsymbol{\theta}\right)$ is the cumulative distribution function of a generalized log-gamma distribution $\operatorname{GLG}(0,1, \lambda)$, more details see section 4.1. If $\lambda>0$, we obtain

$$
\begin{aligned}
\left(\Delta_{\beta \omega}\right)_{i j} & =\left(1-\delta_{i}\right)\left[-\frac{x_{i j}}{\lambda \sigma}+\frac{1}{\lambda \sigma} x_{i j} e^{\lambda \epsilon_{i}}\right]+\delta_{i}\left[x_{i j} \frac{\lambda}{\sigma \Gamma\left(\lambda^{-2}\right)} \frac{a_{i}^{\lambda^{-2}} e^{-a_{i}}}{1-\mathrm{I}\left(\lambda^{-2}, a_{i}\right)}\right] \\
\left(\Delta_{\gamma_{l} \omega}\right)_{i j} & =\left(1-\delta_{i}\right)\left[-\frac{1}{\lambda \sigma} N_{i j l}+\frac{1}{\lambda \sigma} N_{i j l} e^{\lambda \epsilon_{i}}\right]+\delta_{i}\left[N_{i j l} \frac{\lambda}{\sigma \Gamma\left(\lambda^{-2}\right)} \frac{a_{i}^{\lambda^{-2}} e^{-a_{i}}}{1-\mathrm{I}\left(\lambda^{-2}, a_{i}\right)}\right] \\
\left(\Delta_{\sigma \omega}\right)_{i} & =\left(1-\delta_{i}\right)\left[-\frac{1}{\sigma}-\frac{\epsilon_{i}}{\lambda \sigma}+\frac{1}{\lambda \sigma} \epsilon_{i} e^{\lambda \epsilon_{i}}\right]+\delta_{i}\left[\frac{\lambda}{\sigma \Gamma\left(\lambda^{-2}\right)} \frac{\epsilon_{i} a_{i}^{\lambda^{-2}} e^{-a_{i}}}{\left[1-\mathrm{I}\left(\lambda^{-2}, a_{i}\right)\right]}\right] \text { and } \\
\left(\Delta_{\lambda \omega}\right)_{i} & =\left(1-\delta_{i}\right)\left[\frac{1}{\lambda}+\frac{2}{\lambda^{3}} \psi\left(\frac{1}{\lambda^{2}}\right)-\frac{2}{\lambda^{3}}+4 \frac{\log (\lambda)}{\lambda^{3}}-\frac{\epsilon_{i}}{\lambda^{2}}+\frac{2}{\lambda^{3}} e^{\lambda \epsilon_{i}}-\frac{1}{\lambda^{2}} \epsilon_{i} e^{\lambda \epsilon_{i}}\right] \\
& +\delta_{i}\left[-\frac{2 \lambda^{-3} \psi\left(\lambda^{-2}\right) I\left(\lambda^{-2}, a_{i}\right)+\frac{1}{\Gamma\left(\lambda^{-2}\right)} a_{i}^{\lambda^{-2}} e^{-a_{i}}\left(\epsilon_{i}-2 \lambda^{-1}\right)}{1-\mathrm{I}\left(\lambda^{-2}, a_{i}\right)}\right] .
\end{aligned}
$$

If $\lambda<0$, we obtain

$$
\begin{aligned}
\left(\Delta_{\beta \omega}\right)_{i j} & =\left(1-\delta_{i}\right)\left[-\frac{x_{i j}}{\lambda \sigma}+\frac{1}{\lambda \sigma} x_{i j} e^{\lambda \epsilon_{i}}\right]+\delta_{i}\left[-x_{i j} \frac{\lambda}{\sigma \Gamma\left(\lambda^{-2}\right)} \frac{a_{i}^{\lambda^{-2}} e^{-a_{i}}}{\mathrm{I}\left(\lambda^{-2}, a_{i}\right)}\right] \\
\left(\Delta_{\gamma_{l} \omega}\right)_{i j} & =\left(1-\delta_{i}\right)\left[-\frac{1}{\lambda \sigma} N_{i j l}+\frac{1}{\lambda \sigma} N_{i j l} e^{\lambda \epsilon_{i}}\right] \delta_{i}\left[-N_{i j l} \frac{\lambda}{\sigma \Gamma\left(\lambda^{-2}\right)} \frac{a_{i}^{\lambda^{-2}} e^{-a_{i}}}{1-\mathrm{I}\left(\lambda^{-2}, a_{i}\right)}\right] \\
\left(\Delta_{\sigma \omega}\right)_{i} & =\left(1-\delta_{i}\right)\left[-\frac{1}{\sigma}-\frac{\epsilon_{i}}{\lambda \sigma}+\frac{1}{\lambda \sigma} \epsilon_{i} e^{\lambda \epsilon_{i}}\right]+\delta_{i}\left[-\frac{\lambda}{\sigma \Gamma\left(\lambda^{-2}\right)} \frac{\epsilon_{i} a_{i}^{\lambda^{-2}} e^{-a_{i}}}{\left[\mathrm{I}\left(\lambda^{-2}, a_{i}\right)\right]}\right] \text { and } \\
\left(\Delta_{\lambda \omega}\right)_{i} & =\left(1-\delta_{i}\right)\left[\frac{1}{\lambda}+\frac{2}{\lambda^{3}} \psi\left(\frac{1}{\lambda^{2}}\right)-\frac{2}{\lambda^{3}}+4 \frac{\log (\lambda)}{\lambda^{3}}-\frac{\epsilon_{i}}{\lambda^{2}}+\frac{2}{\lambda^{3}} e^{\lambda \epsilon_{i}}-\frac{1}{\lambda^{2}} \epsilon_{i} e^{\lambda \epsilon_{i}}\right] \\
& +\delta_{i}\left[\frac{2 \lambda^{-3} \psi\left(\lambda^{-2}\right) I\left(\lambda^{-2}, a_{i}\right)+\frac{1}{\Gamma\left(\lambda^{-2}\right)} a_{i}^{\lambda^{-2}} e^{-a_{i}}\left(\epsilon_{i}-2 \lambda^{-1}\right)}{\mathrm{I}\left(\lambda^{-2}, a_{i}\right)}\right]
\end{aligned}
$$

where $a_{i}=\lambda^{-2} e^{\lambda \epsilon_{i}}$ and $i=1, \cdots, n ; l=1, \cdots, k$. 


\section{D.1.3 Response perturbation: uncensored case}

For this perturbation scheme we consider that each $y_{i}$ is perturbed as

$$
y_{i}^{\prime}=y_{i}+\omega_{i}
$$

Hence,

$$
\epsilon_{i \boldsymbol{\omega}}=\frac{y_{i}^{\prime}-\mathbf{x}_{i}^{\top} \boldsymbol{\beta}-\mathbf{N}_{i 1} \boldsymbol{\gamma}_{1}-\cdots \mathbf{N}_{i k} \boldsymbol{\gamma}_{k}}{\sigma}
$$

$\omega_{i} \in \mathbf{R}$ and $\boldsymbol{\omega}_{0}=(0, \cdots, 0)^{\top}$, for $i=1, \ldots, n$. So, we obtain

$$
\begin{aligned}
\left(\Delta_{\boldsymbol{\beta} \omega}\right)_{i j} & =\frac{1}{\sigma^{2}} x_{i j} e^{\lambda \epsilon_{i \omega}}, \quad j=1, \cdots, p, \\
\left(\Delta_{\gamma_{l} \omega}\right)_{i j} & =\frac{1}{\sigma^{2}} N_{i j l} e^{\lambda \epsilon_{i \omega}}, \quad j=1, \cdots, q_{l}, \\
\left(\Delta_{\sigma \omega}\right)_{i} & =\frac{1}{\lambda \sigma^{2}}\left[\lambda \epsilon_{i_{\omega}} e^{\lambda \epsilon_{i \omega}}+e^{\lambda \epsilon_{i \omega}}-1\right] \text { and } \\
\left(\Delta_{\lambda \boldsymbol{\omega}}\right)_{i} & =-\frac{1}{\lambda^{2} \sigma}+\frac{1}{\lambda^{2} \sigma} e^{\lambda \epsilon_{i \omega}}-\frac{1}{\lambda \sigma} \epsilon_{i \omega} e^{\lambda \epsilon_{i \omega}},
\end{aligned}
$$

for $i=1, \cdots, n$ and $l=1, \cdots, k$.

\section{D.1.4 Response perturbation: censored case}

If $\lambda>0$, we obtain 


$$
\begin{aligned}
\left(\Delta_{\boldsymbol{\beta} \omega}\right)_{i j} & =\left(1-\delta_{i}\right)\left[\frac{1}{\sigma^{2}} x_{i j} e^{\lambda \epsilon_{i \omega}}\right]+\delta_{i}\left[\frac{-\lambda^{2}}{\sigma^{2} \Gamma\left(\lambda^{-2}\right)} \frac{x_{i j} a_{i}^{\lambda^{-2}} e^{-a_{i}}}{1-\mathrm{I}\left(\lambda^{-2}, a_{i}\right)}\left(a_{i}-\frac{1}{\lambda^{2}}-\frac{a_{i}^{\lambda^{-2}} e^{-a_{i}}}{1-\mathrm{I}\left(\lambda^{-2}, a_{i}\right)}\right)\right] \\
\left(\Delta_{\gamma_{l} \omega}\right)_{i j} & =\left(1-\delta_{i}\right)\left[\frac{1}{\sigma^{2}} N_{i j l} e^{\lambda \epsilon_{i \omega}}\right]+\delta_{i}\left[\frac{-\lambda^{2}}{\sigma^{2} \Gamma\left(\lambda^{-2}\right)} \frac{N_{i j l} a_{i}^{\lambda^{-2}} e^{-a_{i}}}{1-\mathrm{I}\left(\lambda^{-2}, a_{i}\right)}\left(a_{i}-\frac{1}{\lambda^{2}}-\frac{a_{i}^{\lambda^{-2}} e^{-a_{i}}}{1-\mathrm{I}\left(\lambda^{-2}, a_{i}\right)}\right)\right] \\
\left(\Delta_{\sigma \omega}\right)_{i} & =\left(1-\delta_{i}\right)\left[\frac{1}{\lambda \sigma^{2}}\left(\lambda \epsilon_{i_{\omega}} e^{\lambda \epsilon_{\omega}}+e^{\lambda \epsilon_{i \omega}}-1\right)\right] \\
& +\delta_{i}\left[\frac{\lambda^{2}}{\sigma^{2} \Gamma\left(\lambda^{-2}\right)} \frac{a_{i}^{\lambda^{-2}} e^{-a_{i}}}{1-\mathrm{I}\left(\lambda^{-2}, a_{i}\right)}\left[\epsilon_{i}\left(a_{i}-\frac{1}{\lambda^{2}}-\frac{a_{i}^{\lambda^{-2}} e^{-a_{i}}}{1-\mathrm{I}\left(\lambda^{-2}, a_{i}\right)}\right)-\frac{1}{\lambda}\right]\right] \text { and } \\
\left(\Delta_{\lambda \omega}\right)_{i} & =\left(1-\delta_{i}\right)\left[-\frac{1}{\lambda^{2} \sigma}+\frac{1}{\lambda^{2} \sigma} e^{\lambda \epsilon_{i \omega}}-\frac{1}{\lambda \sigma} \epsilon_{i_{\omega}} e^{\lambda \epsilon_{i \omega}}\right] \\
& +\delta_{i}\left[\frac { x _ { i j } a _ { i } ^ { \lambda ^ { - 2 } } e ^ { - a _ { i } } } { \sigma \Gamma ( \lambda ^ { - 2 } ) S ( a _ { i } , \lambda ) } \left[1+\lambda^{-1}\left(\epsilon_{i}-2 \lambda^{-1}\left(\log a_{i}+1\right)\right)+\lambda a_{i}\left(2 \lambda^{-1}-\epsilon_{i}\right)+2 \lambda^{-2} \psi\left(\lambda^{-2}\right)\right.\right. \\
& \left.+\frac{\lambda}{S\left(a_{i}, \lambda\right)}\left(2 \lambda^{-3} \psi\left(\lambda^{-2}\right) I\left(\lambda^{-2}, a_{i}\right)+\frac{1}{\Gamma\left(\lambda^{-2}\right)} a_{i}^{\lambda^{-2}} e^{-a_{i}}\left(\epsilon_{i}-2 \lambda^{-1}\right)\right)\right]
\end{aligned}
$$

And, if $\lambda<0$, we obtain

$$
\begin{aligned}
\left(\Delta_{\beta \omega}\right)_{i j} & =\left(1-\delta_{i}\right)\left[\frac{1}{\sigma^{2}} x_{i j} e^{\lambda \epsilon_{i \omega}}\right]+\delta_{i}\left[\frac{-\lambda^{2}}{\sigma^{2} \Gamma\left(\lambda^{-2}\right)} \frac{x_{i j} a_{i}^{\lambda^{-2}} e^{-a_{i}}}{1-\mathrm{I}\left(\lambda^{-2}, a_{i}\right)}\left(-a_{i}+\frac{1}{\lambda^{2}}-\frac{a_{i}^{\lambda^{-2}} e^{-a_{i}}}{1-\mathrm{I}\left(\lambda^{-2}, a_{i}\right)}\right)\right], \\
\left(\Delta_{\gamma_{l} \omega}\right)_{i j} & =\left(1-\delta_{i}\right)\left[\frac{1}{\sigma^{2}} N_{i j l} e^{\lambda \epsilon_{i \omega}}\right]+\delta_{i}\left[\frac{-\lambda^{2}}{\sigma^{2} \Gamma\left(\lambda^{-2}\right)} \frac{N_{i j l} a_{i}^{\lambda^{-2}} e^{-a_{i}}}{1-\mathrm{I}\left(\lambda^{-2}, a_{i}\right)}\left(-a_{i}+\frac{1}{\lambda^{2}}-\frac{a_{i}^{\lambda^{-2}} e^{-a_{i}}}{1-\mathrm{I}\left(\lambda^{-2}, a_{i}\right)}\right)\right], \\
\left(\Delta_{\sigma \omega}\right)_{i} & =\left(1-\delta_{i}\right)\left[\frac{1}{\lambda \sigma^{2}}\left(\lambda \epsilon_{i_{\omega}} e^{\lambda \epsilon_{i \omega}}+e^{\lambda \epsilon_{i \omega}}-1\right)\right] \\
& +\delta_{i}\left[\frac{\lambda^{2}}{\sigma^{2} \Gamma\left(\lambda^{-2}\right)} \frac{a_{i}^{\lambda^{-2}} e^{-a_{i}}}{1-\mathrm{I}\left(\lambda^{-2}, a_{i}\right)}\left[\epsilon_{i}\left(a_{i}-\frac{1}{\lambda^{2}}-\frac{a_{i}^{\lambda^{-2}} e^{-a_{i}}}{1-\mathrm{I}\left(\lambda^{-2}, a_{i}\right)}\right)-\frac{1}{\lambda}\right]\right] \text { and } \\
\left(\Delta_{\lambda \omega}\right)_{i} & =\left(1-\delta_{i}\right)\left[-\frac{1}{\lambda^{2} \sigma}+\frac{1}{\lambda^{2} \sigma} e^{\lambda \epsilon_{i \omega}}-\frac{1}{\lambda \sigma} \epsilon_{i \omega} e^{\lambda \epsilon_{i \omega}}\right] \\
& +\delta_{i}\left[\frac { x _ { i j } a _ { i } ^ { \lambda ^ { - 2 } } e ^ { - a _ { i } } } { \sigma \Gamma ( \lambda ^ { - 2 } ) S ( a _ { i } , \lambda ) } \left[1+\lambda^{-1}\left(\epsilon_{i}-2 \lambda^{-1}\left(\log a_{i}+1\right)\right)+\lambda a_{i}\left(2 \lambda^{-1}-\epsilon_{i}\right)+2 \lambda^{-2} \psi\left(\lambda^{-2}\right)\right.\right. \\
& \left.\left.+\frac{\lambda}{S\left(a_{i}, \lambda\right)}\left(2 \lambda^{-3} \psi\left(\lambda^{-2}\right) I\left(\lambda^{-2}, a_{i}\right)+\frac{1}{\Gamma\left(\lambda^{-2}\right)} a_{i}^{\lambda^{-2}} e^{-a_{i}}\left(\epsilon_{i}-2 \lambda^{-1}\right)\right)\right]\right]
\end{aligned}
$$

for $i=1, \cdots, n$ and $l=1, \cdots, k$. 


\section{Bibliography}

Agostinelli, C., A. Marazzi, and V. Yohai (2014). Robust estimators of the generalized log-gamma distribution. Technometrics 56, 92-101.

Agostinelli, C., A. Marazzi, and V. Yohai (2017). robustloggamma: Robust estimation of the generalized log gamma model. http://CRAN.Rproject.org/package=robustloggamma .

Akaike, H. (1974). A new look at the statistical identification model. IEEE Transaction Automatic Control 19, 716-723.

Bagdonavicius, V. and M. Nikulin (2001). Accelerated Life Models, Modeling and Statistical Analysis. Chapman and Hall.

Balakrishnan, N. and V. Nevzorov (2003). A Primer on Statistical Distributions. John Wiley and Sons.

Cardozo, C. A., G. A. Paula, and L. Vanegas (2017). sglg: Fitting semiparametric generalized log-gamma regression models. http://CRAN.Rproject.org/package $=$ sglg.

Casella, G. and R. Berger (2002). Statistical Inference. Duxbury.

Chen, J. and H. Rubin (1986). Bounds for the difference between median and mean of gamma and poisson distributions. Statistics and Probability Letters 4 , 281-283.

Colosimo, E. and S. Giolo (2006). Análise de Sobrevivência Aplicada. Edgard Blucher.

Consul, P. and G. Jain (1971). On the log-gamma distribution and its properties. Statistische Theorie 12, 100-106. 
Cook, D. (1986). Assessment of local influence (with discussion). Journal of the Royal Statistical Society 48, 133-169.

Cox, C., H. Chu, M. Schneider, and A. Muñoz (2007). Parametric survival analysis and taxonomy of hazard functions for generalized gamma distribution. Statistics in Medicine 26, 4352-4374.

Crooks, G. (2015). The amoroso distribution. Technical report, Lawrence Berkeley National Laboratory.

De Boor, C. (1978). A Practical Guide to Splines. Springer-Verlag.

DiCiccio, T. (1987). Approximate inference for the generalized gamma distribution. Technometrics 29, 33-40.

Eilers, P. and B. Marx (1996). Flexible smoothing with b-spline and penalties. Statistical Science 11, 89-102.

Fabio, L., G. A. Paula, and M. de Castro (2012). A poisson mixed model with nonnormal random effect distribution. Computational Statistics and Data Analysis 56, 1499-1510.

Ferrari, S. and E. Pinheiro (2012). Small sample likelihood inference in extreme value regression models. Journal of Statistical Computation and Simulation $84,582-595$.

Ferreira, C. and G. Paula (2017). Estimation and diagnostic for skew-normal partially linear models. Journal of Applied Statistics 44, 3033-3053.

Green, P. and B. W. Silverman (1994). Nonparametric Regression and Generalized Linear Models. Chapman and Hall.

Gu, C. and Y. Kim (2002). Penalized likelihood regression: general formulation and efficient approximation. The Canadian Journal of Statistics 30, 619-628.

Hager, H. and L. Bain (1970). Inferential procedures for the generalized gamma distribution. Journal of the American Statistical Association 65, 1601-1609.

Hardle, W. (1990). Applied Nonparametric Regression. Cambrigde University Press.

Hastie, T. and R. Tibshirani (1990). Generalized Additive Models. Chapman and Hall. 
Ibacache-Pulgar, G. and G. A. Paula (2011). Local influence for student-t partially linear models. Computational Statistics and Data Analysis 55, 14621478 .

Ibacache-Pulgar, G., G. A. Paula, and F. Cysneiros (2013). Semiparametric additive models under symmetric distributions. TEST 2Q, 103-121.

Kim, C., B. Park, and W. Kim (2002). Influence diagnostics in semiparametric regression models. Statistics and Probability Letters 60, 49-58.

Lawless, J. (1978). Confidence interval estimation for weibull and extreme value distributions. Technometrics 20, 355-364.

Lawless, J. (1980). Inference in the generalized gamma and log-gamma distributions. Technometrics 22, 409-419.

Lawless, J. (2003). Statistical Models and Methods for Life Time Data. WileyInterscience.

Lehmann, E. and G. Casella (1998). Theory of Point Estimation. SpringerVerlag.

McCullagh, P. and J. A. Nelder (1989). Generalized Linear Models. Chapman and Hall.

Ortega, E., V. Cancho, and G. A. Paula (2009). Generalized log-gamma regression models with cure fraction. Lifetime Data Analysis 15, 79-106.

Ortega, E., G. A. Paula, and H. Bolfarine (2003). Influence diagnostics in generalized log-gamma regression models. Computational Statistics and Data Analysis 42, 165-186.

Ortega, E., G. A. Paula, and H. Bolfarine (2008). Deviance residuals in generalized log-gamma regression models with censored observations. Journal of Statistical Computation and Simulation 78, 747-764.

Ortega, E. and W. Rheinboldt (2000). Iterative Solution of Nonlinear Equations in Several Variables. SIAM.

Paula, G. A. and O. Rojas (1997). On restricted hypothesis in extreme value regression models. Computational Statistics and Data Analysis 25, 143-157. 
Pinhero, E. (2013). Contributions to Inference and Modeling in Extreme Values, in portuguese. Ph. D. thesis, São Paulo University.

Poon, W. and Y. Poon (1999). Conformal normal curvature and assessment of local influence. Journal of the Royal Statistical Society 61, 51-61.

Prentice, R. (1974). A log gamma model and its maximum likelihood estimation. Biometrika 61, 539-542.

Reinsch, C. (1967). Smoothing by spline function. Numerische Mathematik, 170-183.

Relvas, C. and G. A. Paula (2016). Partially linear models with first-order autoregressive symmetric errors. Statistical Papers 57, 795-825.

Rigby, R. A. and D. M. Stasinopoulos (2006). Generalized additive models for location, scale and shape. Applied Statistics 54, 507-554.

Schwarz, G. (1978). Estimating the dimension of a model. The Annals of Statistics 6, 461-464.

Shao, J. (2003). Mathematical Statistics. Springer-Verlag.

Stacy, E. (1962). A generalization of the gamma distribution. Annals of Mathematical Statistics 33, 1187-1192.

Stacy, E. and G. Mihram (1965). Parameter estimation for a generalized gamma distribution. Technometrics 7, 349-358.

Vanegas, L. and G. A. Paula (2015). A semiparametric approach for joint modeling of median and skewness. TEST 24, 110-135.

Vanegas, L. and G. A. Paula (2016). An extension of log-symmetric regression models: R codes and applications. Journal of Statistical Computation and Simulation 86, 1709-1735.

Vanegas, L. and G. A. Paula (2017). Log-symmetric regression models under the presence of non-informative left- or right-censored observations. TEST 26, 405-428.

Wood, S. (2006). Generalized Additive Models, an Introduction with R. Chapman and Hall. 
Yatchew, A. (1998). Nonparametric regression techniques in economics. Journal of Economic Literature 36, 669-721.

Young, D. and S. Bakir (1987). Bias correction for a generalized log-gamma regression model. Technometrics 29, 183-1991.

Zhang, P., P. X. Song, A. Qu, and T. Greene (2008). Efficient estimation for patient-specific rates of disease progression using nonnormal linear mixed models. Biometrics 64, 29-38. 